\title{
DEVELOPMENT OF AN ACOUSTIC VORTICITY METER TO MEASURE SHEAR IN OCEAN-BOUNDARY LAYERS
}

by

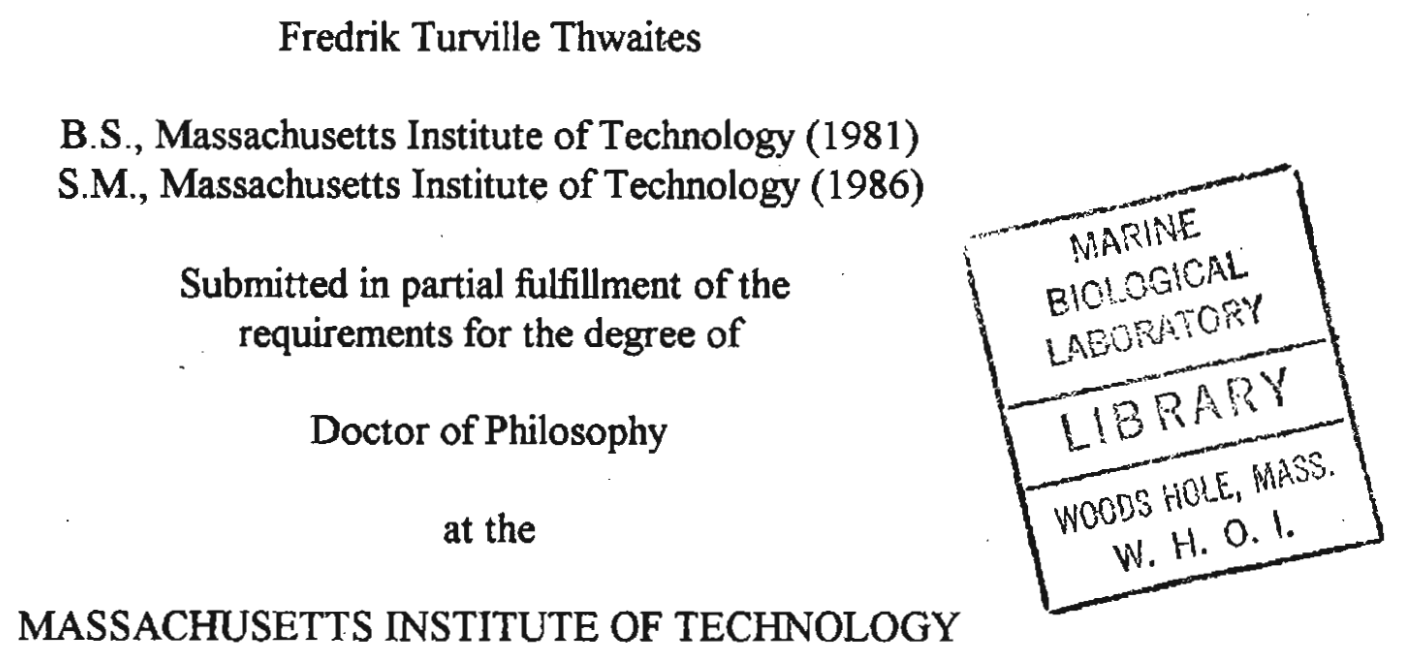

MASSACHUSETTS INSTITUTE OF TECHNOLOGY

and the

WOODS HOLE OCEANOGRAPHIC INSTITUTION

September 1995

C Fredrik T. Thwaites, 1995

The author hereby grants to MIT permission to reproduce and to distribute copies of this thesis document in whole or in part.

Signature of Author

Joint Program in Applied Ocean Science and Engineering Massachusetts Institute of Technology

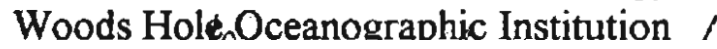

Certified by.

Albert J. Williams, III

Senior Scientist, Woods Hole Oceanographic Institution

Thesis Supervisor

Accepted by 


\title{
DEVELOPMENT OF AN ACOUSTIC VORTICITY METER TO MEASURE SHEAR IN OCEAN-BOUNDARY LAYERS
}

by

\author{
Fredrik Turville Thwaites
}

Submitted to the Department of Mechanical Engineering and to the Joint Committee for Applied Ocean Science and Engineering, Massachusetts Institute of Technology and Woods Hole Oceanographic Institution, September 1995, in partial fulfillment of the requirements for the degree of Doctor of Philosophy.

\begin{abstract}
This thesis describes the analysis and development of an acoustic vorticity meter to measure shear in ocean-boundary layers over smaller measurement volumes than previously possible. A nonintrusive measurement of vorticity would filter out irrotational motion such as surface waves and currents that can swamp small scale measurements of shear. The thesis describes the desired geophysical measurements and translates this oceanographic context into design goals.

The instrument was designed, built, tested, and deployed. It measures three-axis vorticity at 0.83 and 2.45 meters below the ocean surface with measurement volumes of 0.45 meters on a side. The instrument forms a buoy that is inertially instrumented to calculate and remove buoy motion from the measurements. The instrument uses a complementary filter algorithm to estimate attitude and motion from low-power, inexpensive, strapdown rate gyros, accelerometers, and fluxgate magnetometers. The instrument performance has been measured to have a vorticity bias of not more than $1 \times$ $10^{-2}$ per second in a mean flow of 0.7 meters per second, a bias of not more than $1 \times 10^{-2}$ per second in the down-wave and vertical directions in typical ocean waves, and a 30 decibel spectral rejection of surface wave velocity.

Two instrument deployments are described to show the potential of the system. The instrument has measured shear in the upper-ocean-boundary layer, and these measurements are compared to concurrently measured wind stress and stratification. The instrument was also deployed, tethered in the thermocline, in an area of high internal wave activity. Richardson-number time series were measured and compared favorably to concurrently measured Richardson numbers made over a larger spatial scale.
\end{abstract}

Thesis Supervisor: Albert J. Williams III.

Title: Senior Scientist 


\section{Acknowledgments}

I would like to thank the members of my thesis committee; Eugene Terray, John Trowbridge, Robert Weller, David Wilson, and Albert Williams III; for their helpful suggestions for improving the clarity of the text and for serving on my committee. Particular thanks go to Albert J. (Sandy) Williams III whose experience, good humor, and generous help greatly assisted me in this work.

The engineers, machinists, welders, and technicians at WHOI are also recognized for their skills, judgement, and work in helping turn an idea into a practical working instrument. Particular thanks go to Martin Woodward (Woody), Gary Stanbrough, Terrence Rioux, Ken Doherty, and Richard Koehler.

Jim Edson and Jeff Hare made the windstress measurements during the Buzzards Bay Deployment. I thank the Parson's Lab at MTT for the use of a wave tank and laser doppler velocimeter. I thank the Coastal Research Center for the use of their shop. The new Bedford Regional Vocational School built a large tank that I used for testing and calibrating the instrument. I thank Laura Predario for reading the thesis and providing editing advice. I thank Markku Santala and Todd Morrison for help in the lab.

Support for this project was received from National Science Foundation grants OCE-9018623 and OCE-9314357, Office of Naval Research grant NO0014-89-J-1058, and a Keck Foundation instrumentation initiative grant.

I thank my wife Laurie and my parents who sustained me through this work. 


\section{TABLE OF CONTENTS}

Page No.

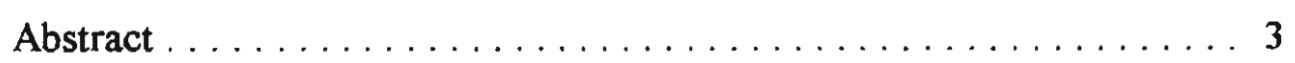

Acknowledgments $\ldots \ldots \ldots \ldots \ldots \ldots \ldots \ldots \ldots \ldots \ldots \ldots \ldots$

List of Figures and Tables $\ldots \ldots \ldots \ldots \ldots \ldots \ldots$

$\begin{array}{ll}\text { Chapter 1. Introduction } & 13\end{array}$

1.A. Ocean Boundary Layers . . . . . . . . . . . . . 13

Upper-Boundary Layer . . . . . . . . . . . . . . . 14

Free Surface Considerations . . . . . . . . . 16

Sensor-Wave-Correlation Bias . . . . . . . . . 18

Reasons to Measure Eulerian Shear ......... 18

Measurement Types . . . . . . . . . . . . . 19

Internal-Boundary Layer $\ldots \ldots \ldots \ldots \ldots \ldots \ldots$

Bottom-Boundary Layer $\ldots \ldots \ldots \ldots \ldots \ldots \ldots$

1.B. Previous Work . . . . . . . . . . . . . . . 21

1.C. Instrument Design Goals : . . . . . . . . . . . . . . 21

$\begin{array}{ll}\text { Chapter 2. Instrument Design } & 23\end{array}$

2.A. Mechanical Design Overview ............... 23

Vortex Shedding .................... 29

Material Selection .................... 30

Transducers and their Mounting $\ldots \ldots \ldots \ldots \ldots \ldots 31$

Buoy Design $\ldots \ldots \ldots \ldots \ldots \ldots \ldots \ldots \ldots \ldots \ldots \ldots \ldots \ldots \ldots \ldots$ 
2.B. Electrical Design ... . . . . . . . . . . . . 37

Electrical Overview . . . . . . . . . . . . . 37

Inertial Measurement Unit . . . . . . . . . . . . . 40

Calibration of the Inertial Measurement Unit . . . . . . . 42

2.C. Signal Processing $\ldots \ldots \ldots \ldots \ldots \ldots \ldots \ldots \ldots \ldots$

Inertial Processing Background . . . . . . . . . . . 47

Coordinate Transformations . . . . . . . . 47

Euler Angle Choice . . . . . . . . . . . . . . . . . 49

Other Techniques to Calculate Attitude ...... 51

Magnetic Compasses . . . . . . . . . . . . 53

Coning Motions $\ldots \ldots \ldots \ldots \ldots \ldots \ldots$

Algorithm Error Propagation . . . . . . . . . . 56

Inertial Algorithm . . . . . . . . . . . . . 60

Constraints of Buoy Motion . . . . . . . . 60

Complementary Filters . . . . . . . . . . . . 60

Model of Wave Motion Caused Angle Error . . . . . 62

Horizontal Reference Frame for Heading . . . . . . 64

Inertial Algorithm . . . . . . . . . . 65

Data Processing ...................67

Simulation of Inertial Processing $\ldots \ldots \ldots \ldots \ldots$

General Accuracy Results of Simulations . . . . . . 71

Intermediate Reference Frame for Heading . . . . . 72

Rejection of Coning Motion . . . . . . . . . . 73 
Low Frequency Errors . . . . . . . . . . . . . 73

Algorithm Error Breakdown . . . . . . . . . . . 77

Simpler Inertial Algorithms . . . . . . . . . 8 81

$\begin{array}{lr}\text { Chapter 3. Performance } & 85\end{array}$

3.A. Modeling Sensor Errors . . . . . . . . . . . . . . . 85

3.B. Measurements of Performance ................ 94

Electronic Noise . . . . . . . . . . . . . . . . . . . 95

Electronic Zero Drift . . . . . . . . . . . . 96

Sound Speed Changes . . . . . . . . . . . . . . . 96

Thermistor Response Time . . . . . . . . . . . . . 96

Bias in Constant Flow . . . . . . . . . . . . . . . 97

Wake Error Cancellation . . . . . . . . . . 97

Measured Bias in Constant Flow . . . . . . . . . . . 99

Variance in Constant Flow . . . . . . . . . . . . . . 102

Bias in Wave Flow . . . . . . . . . . . . . . . 105

Rotation Arm Bias . . . . . . . . . . . . . 106

Wave Bias Sensitivity to Orientation . . . . . . 113

Open-Ocean Bias Measurement . . . . . . . . . 115

Wave Spectral Rejection of Vorticity Sensors . . . . . . 121

Laboratory-Wave Spectral Rejection ........ 121

Ocean-Wave Spectral Rejection ........... 123

Buoy Flexing $\ldots \ldots \ldots \ldots \ldots \ldots \ldots \ldots \ldots$

$\begin{array}{lr}\text { Chapter 4. Applications } & 129\end{array}$ 
4.A. Upper-Boundary-Layer Shear . . . . . . . . . . . . . . . . . 129

4.B. Thermocline Richardson Number . . . . . . . . . . . 136

$\begin{array}{ll}\text { Chapter 5. Summary } & 139\end{array}$

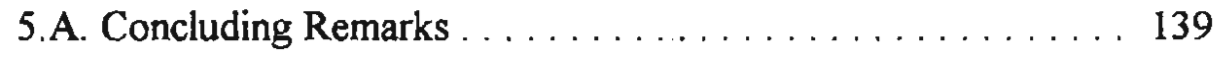

5.B. Future Work . . . . . . . . . . . . . . . . 141

Appendix A. Mechanical Drawings . . . . . . . . . . . . . 142

Appendix B. Processing Code . . . . . . . . . . . . . . . . . . 152

Appendix C. Rotating Arm Measured Vorticity Bias .......... 165

Appendix D. Upper-Boundary-Layer Shear in Wind Coordinates . . . . 171

References .............................. 177 


\section{LIST OF FIGURES AND TABLES}

Page No.

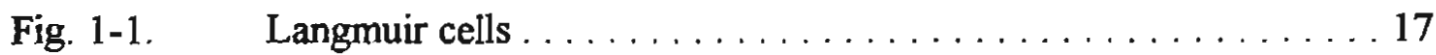

Fig. 2-1. Circulation error from transducer wakes with triangular . . . . . . 24 and square geometries

Fig. 2-2. Velocity cancellation in constant flow . . . . . . . . 25

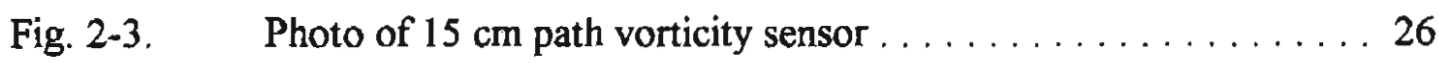

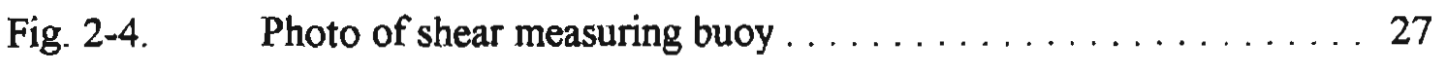

Fig. 2-5, Relative beam patterns for transducers . . . . . . . . . . 32

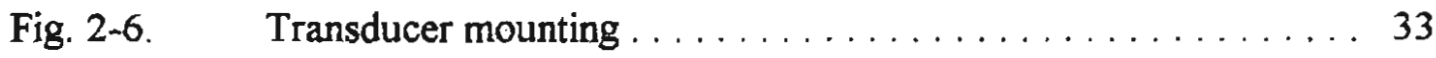

Fig. 2-7. Thermocline shear measuring drifter . . . . . . . . . . 36

Fig. 2-8. Photo of electronics . . . . . . . . . . . . . . 37

Fig. 2-9. Block diagram of electrical system . . . . . . . . . 38

Table 2-1. Vorticity meter data format . . . . . . . . . . . . . . . . 39

Table 2-2. Inertial measurement unit sensors $\ldots \ldots \ldots \ldots \ldots \ldots \ldots \ldots$. . . 41

Fig. 2-10. a. Calibration of accelerometers . . . . . . . . . . . . 43

Fig. 2-10. b. Calibration of rate gyros . . . . . . . . . . . . . . . . 44

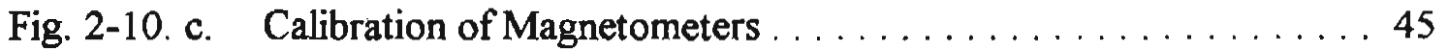

Fig. $2-11 . \quad$ y axis rate gyro noise $\ldots \ldots \ldots \ldots \ldots \ldots \ldots \ldots \ldots \ldots \ldots \ldots$

Table 2-3. Inertial sensor noise standard deviations . . . . . . . . . 46

Fig. 2-12. Rotation of coordinate system $x y z$ to $x^{\prime} y^{\prime} z^{\prime} \ldots \ldots \ldots \ldots \ldots$. . . 47

Fig. 2-13. 3,2,1 Euler angle system order of rotations . . . . . . . . . . 49

Fig. 2-14. Coordinate system used in compass example . . . . . . . . 53

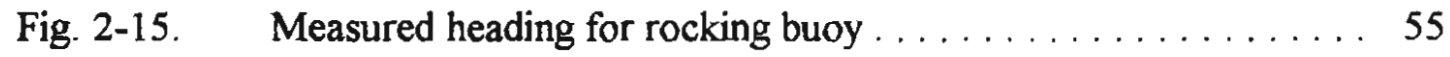

Fig. 2-16. Error progression in coning motion . . . . . . . . . . . . 59

Fig. 2-17. Complementary filter of redundant sensor measurements . . . . . 61

Fig. 2-18. Conceptual block diagram of inertial processing . . . . . . . 62

Fig. 2-19. Angle noise for 2D model with Pierson-Moskowitz spectrum . . . 64

Fig. 2-20. Block diagram of Euler angle algorithm . . . . . . . . 66 
Fig. 2-21. Vorticity meter data path $\ldots \ldots \ldots \ldots \ldots \ldots \ldots \ldots \ldots \ldots$

Fig. 2-22. Algorithm to compute earth referenced flow velocity . . . . . . . 69

Fig. 2-23. Inertial processing simulation block diagram $\ldots \ldots \ldots \ldots \ldots$

Fig. 2-24. East and vertical velocity spectral densities from deployment . . . . 74 south of Martha's Vineyard

Fig. 2-25. Simulated east velocity spectra . . . . . . . . . . . 76

Table 2-4. Array of simulations used to infer size of error terms . . . . . . 78

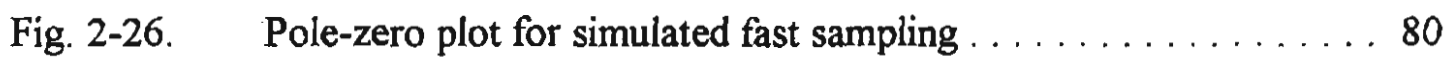

Table 2-5. Simulations of simpler inertial algorithms $\ldots \ldots \ldots \ldots \ldots . \ldots 2$

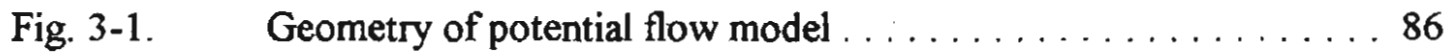

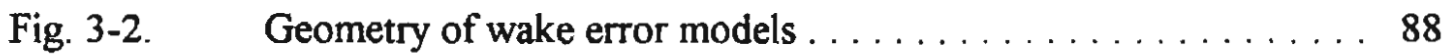

Fig. 3-3. Wake model for center stalk and pod wake bias .......... 89

Fig. 3-4. Center stalk model used on tow tank data .............. 91

Fig. 3-5. Geometry of lift related circulation model . ............ 93

Fig. 3-6. Current measured in a bucket . . . . . . . . . . . . . 95

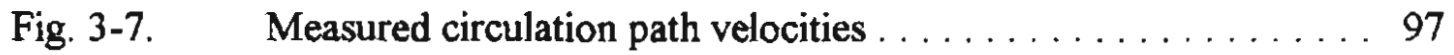

Fig. 3-8. Vorticity bias for triangular circulation path . . . . . . . . . 99

Fig. 3-9. Uncorrected vorticity bias in constant flow . . . . . . . . . 100

Fig. 3-10. Corrected vorticity bias in constant flow . . . . . . . . . . 100

Table 3-1. Vorticity sensor bias in $0.643 \mathrm{~m} / \mathrm{sec}$ constant flow .......... 101

Fig. 3-11. Geometry used to measure shed vortex wake ........... 102

Fig. 3-12. Fluctuating measured velocity perpendicular to center stalk . . . 103

Fig. 3-13. Spectral densities of cross and parallel velocities of $45 \mathrm{~cm} \ldots \ldots 104$ path sensor

Fig. 3-14. Spectral densities of measured cross velocities at different . . . . 104 carriage speeds

Fig. 3-15. Vorticity means from rotating arm test . . . . . . . . . . . 107

Fig. 3-16. Down-wave vorticity means . . . . . . . . . . . . . . . 108

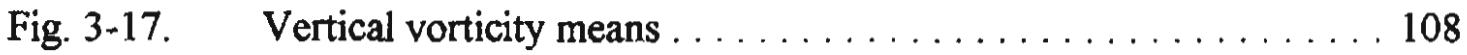

Fig. 3-18. Autocorrelation of vorticity .................. 109 
Fig. 3-19. Rotating arm sensor means plotted with respect to . . . . . . . 110 relative drift velocity

Fig. 3-20. Rotating arm vorticity means for constant . . . . . . . . 111 drift-velocity-divided-by-wave velocity

Fig. 3-21. Horizontal acceleration spectra from rotating arm test . . . . . . 112

Fig. 3-22, Open-ocean deployment acceleration spectra . . . . . . . . 112

Fig. 3-23. CCIW wave tank flow measurements . . . . . . . . . . 113

Table 3-2. Mean vorticity in tank coordinates at different sensor rotations . . 115

Fig. 3-24. Mean vorticity measured by shallow sensor south of . . . . . . 116 Martha's Vineyard

Fig. 3-25. Mean vorticity measured by deep sensor south of $\ldots \ldots \ldots 116$ Martha's Vineyard

Fig. 3-26. Directional wave spectrum south of Martha's Vineyard . . . . . . 117

Fig. 3-27. Coordinate rotation to wave coordinates . . . . . . . . 117

Table 3-3. Mean vorticity in wave coordinates $\ldots \ldots \ldots \ldots \ldots \ldots$

Fig. 3-28. Vertical vorticity in buoy heading bins . . . . . . . . . . . . . 119

Fig. 3-29. Horizontal vorticity in buoy heading bins . . . . . . . . . 120

Fig. 3-30. Wave tank time series of velocity, shear, and circulation over . . . 121 path length

Fig. 3-31. Vorticity meter spectral rejection of waves . . . . . . . . 122

Fig. 3-32. $\quad 1.5 \mathrm{~m}$ path benthic vorticity meter spectral rejection of waves $\ldots 123$

Fig. 3-33. Measured spectral density of velocity . . . . . . . . . 125

Fig. 3-34. Enlarged view of measured spectral peak . . . . . . . . . 125

Table 3-4. Major sensor error sources . . . . . . . . . . . . . 127

Fig. 4-1. Buzzards Bay deployment shears, measured and estimated from . . 130 wind stress

Fig. 4-2. Vertical velocity spectra from Buzzards Bay . . . . . . . . 131

Fig. 4-3. Buzzards Bay deployment gradient Richardson numbers . . . . . 132

Fig. 4-4. Wave velocity directional spectrum from Buzzards Bay . . . . . 133

Fig. 4-5. Vorticity from shallow sensor in Buzzards Bay . . . . . . . . . 134 
Fig. 4-6. Vorticity from deep sensor in Buzzards Bay . . . . . . . 135

Fig. 4-7. Massachusetts bay thermocline gradient Richardson numbers ... 137

Fig. 4-8. Histogram of Richardson numbers measured over two length scales 138

Fig. A-1. Shear measuring buoy assembly . . . . . . . . . . . 143

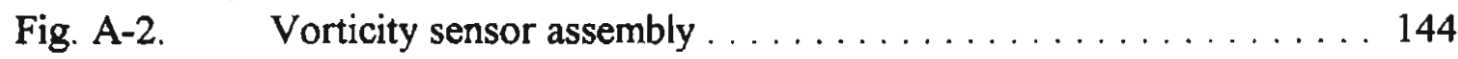

Fig. A-3. Pressure vessel assembly . . . . . . . . . . . . 145

Fig. A-4. Lower hub detail . . . . . . . . . . . . . . . . . . . . . . 146

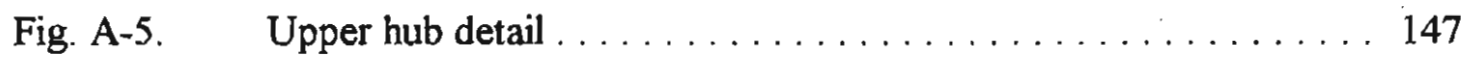

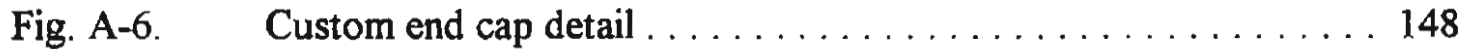

Fig. A-7. Instrument electronics case detail . . . . . . . . . . . . . 149

Fig. A-8. Transducer mount detail . . . . . . . . . . . . . 150

Fig. A-9. Central stalk, pod arms, and braising jig . . . . . . . . 151

Table B-1. Data Variables ......................... 152

Fig. C-1. Vorticity means in constant flow . . . . . . . . . . . 166

Fig. C-2. Vorticity means at 10 second rotation ............. 167

Fig. C-3. Vorticity means at 7 second rotation . . . . . . . . . . . 167

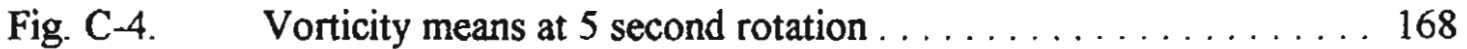

Fig. C-5. Vorticity means at 3.8 second rotation . . . . . . . . . 168

Fig. C-6. Vorticity means of $15-\mathrm{cm}$ path sensor . . . . . . . . . . 169

Fig. C-7. Buoy response functions $\ldots \ldots \ldots \ldots \ldots \ldots \ldots \ldots \ldots$

Fig. D-1. Windstress coordinate system ................ 171

Table D-1. Santala upper-boundary-layer shear model ........... 172

Fig. D-2. Santala model layer transition depths ............... 173

Fig. D-3. Downwind shear from vorticity and windstress $0.83 \mathrm{~m}$ depth . . . 174

Fig. D-4. Downwind shear from vorticity and windstress $2.45 \mathrm{~m}$ depth . . . 174

Fig. D-5. Stratification in lower sensor and between sensors . . . . . . . 175

Fig. D-6. Crosswind shear from vorticity and windstess for both sensors . . 176 


\section{CHAPTER 1. INTRODUCTION}

This thesis documents the development and testing of an instrument system to measure ocean vorticity and shear in the upper, internal, and bottom-boundary layers. The purpose of this project has been to provide ocean scientists with a tool capable of measuring ocean shear with finer resolution and or closer to the boundaries. The deployment data are presented to demonstrate the instrument's potential rather than to contribute directly to understanding marine boundary layers.

This chapter describes the geophysical environment to be measured, why vorticity is used to measure shear, how shear can be used to measure vertical diffusivity, and reviews the tradeoff between resolution and accuracy in a shear or vorticity measurement. The upper-boundary layer, internal-boundary layer, and bottom-boundary layer are reviewed to motivate their measurement and to define the instrument design goals. Chapter two describes the mechanical design, electrical design, the inertial measurement unit and its inertial processing, and the signal processing done on the raw data. Chapter three models and measures the sensor performance. Chapter four describes two deployments of the instrument: one in the upper boundary layer to measure shear, and one in the thermocline to measure gradient Richardson number. Chapter five concludes the thesis and discusses future work and improvements to the system

\section{A. OCEAN BOUNDARY LAYERS}

Boundary layers mediate the turbulent fluxes of heat, momentum and chemical species. Turbulent transport dominates molecular diffusion everywhere except in diffusive sublayers on the order of one millimeter thick. The water side of the air-sea interface controls the air-sea transfer of most gasses (Kitaigorodskii and Donelan, 1984). Gas transfer resistance is a function of turbulence in the boundary layer. Many quantities in ocean boundary layers such as Reynolds stress, chemical flux, or shear are difficult to measure in the presence of gravity-wave velocities that swamp turbulent velocities. 
Typical boundary-layer flows have wave velocities of order $0.5 \mathrm{~m} / \mathrm{s}$ and shear of order 2 * $10^{-2}$ per second. Over large measurement separations ie. ten meters, this shear is readily measured. Yet over 0.5 meters, if the shear is to be measured with current meters, the meter's accuracy would need to be better than one percent. In the presence of waves this accuracy is not readily achievable. This difficulty results in a shortage of near-surface measurements of shear and poorly-calibrated-closure relations for ocean and climate models. Vorticity can be measured as a surrogate for shear because time-average, areaaverage, horizontal vorticity equals time-average, area-average, vertical shear in oceanboundary layers. A nonintrusive measurement of vorticity would measure wind-driven shear and automatically remove irrotational surface-gravity-wave and current velocities, allowing measurement of shear.

This thesis will emphasize measurements for studies of vertical diffusivity. For readers not too familiar with geophysical flows, vertical (diapycnal) diffusivity is orders of magnitude smaller than horizontal (isopycnal) diffusivity. In the open ocean, far away from boundaries, a typical horizontal diffusivity is $3 \mathrm{~m}^{2} / \mathrm{s}$ while the vertical diffusivity is just $1 * 10^{-5} \mathrm{~m}^{2} / \mathrm{s}$ (Ledwell, Watson, and Law, 1993). Density structure clearly plays a large role in ocean diffusivity.

\section{UPPER-BOUNDARY LAYER}

The measurement of upper-boundary-layer shear is the focus of this instrument development. The ocean-upper-boundary layer is often compared to the well studied and relatively well understood unstratified turbulent flow over a rigid wall. Factors that can complicate the ocean surface are waves, wave breaking, stratification, rotation, and organized motions such as Langmuir cells.

Shear in a constant stress layer in an unstratified turbulent flow next to a rigid wall, is given by equation (1-1) for distances greater than $\frac{z u_{*}}{v}>50$ from the wall, of order $5 \mathrm{~mm}$ in the ocean (Monin and Yaglom, 1987). In this equation, $\frac{\partial u}{\partial z}$ is Eulerian shear, $u_{*}$ is the friction velocity $\sqrt{\frac{\tau}{\rho}}$ the square root of the shear stress divided by density, 


$$
\frac{\partial u}{\partial z}=\frac{u_{*}}{\kappa z}
$$

$\kappa$ is von Karmon's constant usually assumed to be 0.4 , and $z$ is the distance from the wall. This shear will give a logarithmic velocity profile, equation (1-2), and the dissipation will

$$
u(z)=\frac{u_{*}}{\kappa} \ln (z)+\text { const }
$$

be $\frac{u_{*}^{3}}{K z}$ (Tennekes and Lumley, 1989). The assumptions for this log layer are constant shear stress, constant density and a Reynolds number high enough that viscous forces are negligible compared to turbulent Reynolds stress. By comparing measured ocean shear to measured windstress, the effects on vertical mixing effectiveness of stratification, wave breaking, surfactants, and Langmuir cells can be measured.

Stratification can inhibit turbulence. To show how this happens, the turbulent kinetic energy budget equation will be reviewed, equation (1-3) (Stull, 1988). In this

$$
\begin{gathered}
\left(\frac{\partial}{\partial t}+\bar{u} \frac{\partial}{\partial x}+\bar{w} \frac{\partial}{\partial z}\right) \overline{q^{\prime}}=-\overline{u^{\prime} w^{\prime}} \frac{\partial \bar{u}}{\partial z}-\frac{\partial}{\partial z}\left(\frac{\overline{p^{\prime} w^{\prime}}}{\rho}+\frac{\overline{q^{2} w^{\prime}}}{2}\right)-\varepsilon-\frac{g}{\rho_{o}} \overline{\rho^{\prime} w^{\prime}} \\
\text { term: (a) } \\
(b)
\end{gathered}
$$

equation, term $a$ is the material derivative of turbulent kinetic energy $q^{, 2}=u^{, 2}+v^{, 2}+w^{, 2}$, term $b$ is creation of turbulent energy by shear, term $c$ is pressure diffusion, term $d$ is energy diffusion, term $e$ is viscous dissipation, and term $f$ is destruction by buoyancy. In a steady-state, horizontally-uniform, boundary layer, term $\alpha$ is assumed zero and the transport terms $c$ and $d$ are assumed very small leaving equation (1-4). The energy destruction-by-buoyancy term takes energy away from turbulence. 


$$
-\overline{u^{\prime} w^{\prime}} \frac{\partial \bar{u}}{\partial z} \approx \varepsilon+\frac{g}{\rho_{o}} \overline{\rho^{\prime} w^{\prime}}
$$

The wind driven current as a function of depth is a function of the vertical-eddy diffusivity, which in turn is strongly influenced by stratification (Price, Weller, and Schudlich, 1987). If a constant wind stress and a constant vertical-eddy diffusivity with depth is assumed, the momentum balance, including Coriolis force, generates an Ekman spiral. If eddy diffusivity is assumed to be proportional to depth, a different result is obtained which is closer to most ocean measurements of current shear (Madsen, 1977). The solution is a function of the vertical eddy diffusivity, which in turn is a function of stratification and any other modifications to the turbulence such as wave breaking energy addition, or Langmuir cells. The instrument developed in this thesis measures shears close to the ocean surface, in the upper three meters, where there are few shear measurements.

\section{FREE SURFACE CONSIDERATIONS}

Free-surface considerations that can be important to understanding the oceanupper-boundary layer include Langmuir cells, a reduced-shear layer (as compared to the turbulent wall layer model), gas transfer, wave breaking and their resultant bubbles and droplets, and surfactants.

Langmuir cells are three-dimensional drift currents that form counter-rotating helical vortices parallel to the wind, Fig 1-1 (Langmuir, 1938). Langmuir cells are often identified by windrows of debris floating parallel to the wind, and arranged by convergence zones of the vortices. When these cells exist, they dominate vertical mixing over their extent (Gordon, 1970). While cell-averaged shears are of order $1 \times 10^{-2} / \mathrm{s}$, shear is concentrated on the edges of the down welling jets and can be significant compared to average current shear. Langmuir cells are important in understanding the upper-boundarylayer dynamics and complicate measurements in this environment by making time-average shear and vorticity horizontally nonuniform. 


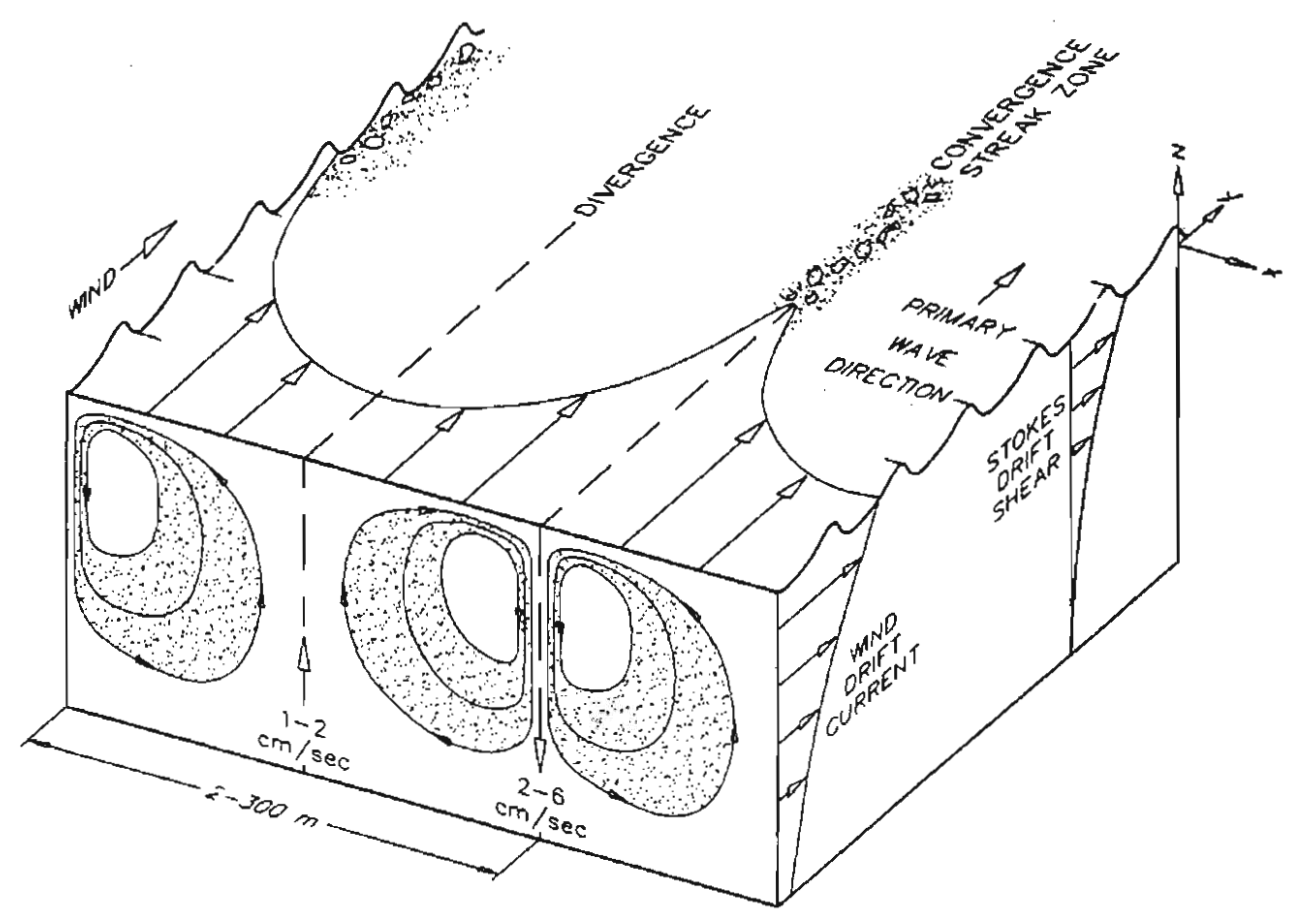

Figure 1-1. Artists conception of Langmuir cells

The transfer of momentum from wind to waves and current is not well understood and is an area of active research. While the wind's momentum is transferred initially largely to waves, and most of this to short wavelets, most of this momentum is transferred through breaking to Eulerian shear and currents. Less than six percent of the transferred momentum eventually is radiated away as waves (Mitsuyasu, 1985). Many researchers have found a layer of reduced shear and enhanced dissipation (some by a factor of 100) as compared to a wall layer (Csanady, 1983 and 1984, Cheung and Street, $1988 \mathrm{a}$ and b, Agrawal et al, 1992). Santala (1991) reports a zone of no shear in the direction of windstress to a depth of $\frac{g z}{u^{2}} \approx 1.2 * 10^{5}$ and significant shear at right angles to the windstress. One of the gơals in developing the vorticity meter is to be able to make better open-ocean measurements of shear close enough to the surface to study this reduced shear phenomenon. 
Research on gas transfer between the ocean and atmosphere has received increased interest with the concern over atmospheric carbon dioxide buildup. The transfer of lowsolubility gases is controlled by the water-side diffusive sublayer, which is of order one millimeter thick (Kitaigorodskii and Donelan, 1984). Straining and renewal of the diffusive sublayer by upper-boundary-layer turbulence is a major contributor to gas transfer (Brumley and Jirka, 1988). Bubbles from wave breaking can significantly increase gas transfer when the wind exceeds some velocity (Broecker and Siems, 1984). In addition to their increased effective-surface area for diffusion, bubbles add turbulence and strain the diffusive sublayer.

The presence of surfactants affect the surface boundary condition, dampen capillary waves, and reduce gas transfer (Hunt, 1984). While surfactants reduce the surface drag coefficient, Wu (1983) reports that surfactants can still increase surface drift currents.

\section{SENSOR-WAVE-CORRELATION BIAS}

Sensor-wave-correlation bias of velocity and velocity-derived-shear measurements is caused by a correlation between sensor motion and the wave-field-velocity gradient (Pollard, 1973). It is different from Stokes drift and Stokes-drift shear because sensors and buoys do not exactly follow water motion. In open-ocean fetch conditions, this bias is greater than wind-driven Eulerian shear (Wu, 1975). Santala (1991) and Santala and Terray (1992) derived an algorithm for removing this bias from measurements taken from an inertially instrumented buoy, but the accuracy of this bias removal is limited by the accuracy of the measurements of the directional-wave spectrum. A nonintrusive measurement of vorticity, on the other hand, is not effected by any motion-correlation bias.

\section{REASONS TO MEASURE EULERIAN SHEAR}

In addition to measuring Eulerian shear to understand the structure of surface boundary layers, one reason for measuring Eulerian shear is to be able to determine the 
effective turbulent diffusivity. Comparing wind stress to Eulerian shear results in a measurement of the vertical-eddy diffusivity, and by the Reynolds analogy for turbulent diffusion, is also a measurement of the effectiveness of turbulent transfer of heat and solutes (Rohsenow and Choi, 1961). This measurement of turbulence, although indirect, is better conditioned than direct measurements of Reynolds stress near the ocean surface because of the very large quadrature components from wave velocities that do not contribute to the vertical flux of momentum, heat or solutes. Any wave reflections (standing waves) or phase lag between velocity measurement axes, dooms a measurement of Reynolds flux in the wave field.

\section{MEASUREMENT TYPES}

Different methods for measuring drift currents and shear include Lagrangian drifters, Eulerian measurements, and surface-referenced buoys. For measuring mass transport velocities, Lagrangian drifters seem like an obvious inexpensive option. The problems with drifters include uncalibrated drift relative to their target depth (Geyer, 1989), and their tendency to get stuck in convergence zones of three-dimensional flow structures such as Langmuir cells, which can have unrepresentative drift velocities. If measurement of Eulerian shear is desired, the correlation bias of sensor-wave motion of the drifters would have to be compensated for, which would require inertial instrumentation and measuring relative velocity, significantly increasing the drifter's cost and complexity. An Eulerian (fixed in space) measurement of velocity or shear requires a tower which is expensive, has a large flow obstruction, and cannot be used in deep water. Three-point moorings and taut moorings are not stiff enough to make Eulerian-velocity measurements free of motion-correlation bias. Surface-referenced buoys are often used to measure ocean currents but buoy motion must be known in order to remove wave bias (Santala, 1991 and Santala and Terray, 1992). Buoys ride with swell, giving a nonEulerian reference frame. This swell-based reference frame can actually assist near-surface measurements, allowing velocity measurements within a waveheight of the surface (Cheung and Street, 1988). Measuring vorticity from a surface buoy avoids motioncorrelation bias, but the buoy must be inertially instrumented to remove buoy motion from 
the buoy's measurements. If the buoy motion is not known and not compensated for, motions such as coning motions could result in time average sensor measurements that are caused by the buoy motion and do not exist in the fluid. Coning motions will be explained in the inertial processing section.

\section{INTERNAL BOUNDARY LAYER}

Density-stratified layers in the ocean inhibit turbulent-vertical mixing, support internal waves, and can be treated as a boundary layer (Salmon, 1990). The gradient Richardson number equation (1-5) is a ratio of the relative strengths of stratification,

$$
R i=\frac{N^{2}}{\left(\frac{d U}{d z}\right)^{2}}=\frac{-\frac{g}{\rho_{o}} \frac{d \rho_{r}}{d z}}{\left(\frac{d U}{d z}\right)^{2}}
$$

which inhibits mixing, and shear, which encourages mixing (Tumer, 1973). In this equation, $N$ is the Brunt-Vaisala frequency, $\frac{d U}{d z}$ is the vertical shear, $g$ is gravity, $\rho_{0}$ is average density, and $\frac{d \rho_{r}}{d z}$ is the density gradient. A gradient Richardson number less than one quarter is a necessary, and in practice usually sufficient, condition for turbulent vertical mixing to occur. Internal waves have vorticity in a density-stratified layer. Internal-wave-shear instability is thought to be the major source of vertical mixing in density-stratified layers of the ocean away from boundaries; therefore accumulating more gradient-Richardson-number statistics in the ocean over different spatial scales will help in further understanding vertical mixing in the ocean (Gargett et al, 1981, and Gargett and Holloway, 1984).

\section{BOTTOM-BOUNDARY LAYER}

Understanding turbulent transport and stress in the bottom-boundary layer is necessary to understand processes of sediment transport. Measurement of bottomboundary-layer turbulence near shore is complicated by surface wave swell that reaches 
the bottom, is often much more energetic than the turbulence, and occupies the same frequency band (Grant and Madsen, 1986). The ability to measure turbulence, free of surface swell, should assist ocean scientists in studying nonlinear wave-current interaction. By measuring vorticity, the surface swell is filtered out leaving the turbulence to be more readily measured.

\section{B. PREVIOUS WORK}

Other researchers have made single-axis vorticity measurements in laboratories and in the ocean. Rossby (1975) proposed measuring ocean vorticity and made a laboratory demonstration of single axis vorticity measurement by measuring the circulation around a closed triangle by measuring the difference in acoustic travel time. The lab demonstration used acoustic mirrors to form the circulation triangle. He proposed measuring circulation around a large triangle of from 3 kilometers on a side to ocean-basin size. Tsinober, Kit, and Teitel (1986) measured single-axis vorticity electromagnetically in a lab over very small scale. They made a seven electrode probe to make a central difference approximation of the divergence of the electric potential which is proportional to fluid vorticity. Their probe was only 2 millimeters across. Muller, Lien, and Williams (1988) estimated relative vorticity in the ocean from current meters that were trimoored in the ocean thermocline. The current meters formed horizontal triangles from 8.5 to 1600 meters on a side and the measurements were not compensated for mooring motion. Menemenlis and Farmer (1992) measured vertical vorticity 8 and 20 meters beneath the arctic ice sheet acoustically. They measured circulation around a triangle 200 meters on a side. Tom Sanford (APLUW, personal communication) has measured small-scale, singleaxis vorticity in ocean boundary layers by measuring the divergence of the electric field.

The instrument whose development is described in this thesis, has made the first three-axis vorticity measurements in ocean boundary layers.

\section{C. INSTRUMENT DESIGN GOALS}

The primary goal in developing this instrument is to have the ability to measure 
Eulerian shear in the ocean-upper-boundary-layer, over finer resolution and closer to the surface, than previously practical. The system should be deployable by a moderately sized oceanographic vessel such as the $R$. V. Asterias (a 15 meter workboat), and the measurement volumes should be scaled and at a depth to measure the reduced shear layer. Below a depth of about 5 meters, moored current meters are adequate for measuring shear. This instrument measures shear in the upper 5 meters of the ocean and can measure shear in the upper meter of the ocean in order to measure the reduced shear layer. A secondary goal for the system is that it be able to measure internal-wave shear and stratification in the internal-boundary layer (thermocline) over small scales in the presence of surface swell. An ancillary use for these sensors was to measure turbulence in the bottom-boundary layer in the presence of surface swell. This later application of the vorticity meter is not covered in detail in this thesis, but data from this application is presented to show swell-spectral rejection in a coastal deployment. 


\section{CHAPTER 2. INSTRUMENT DESIGN}

Chapter two describes several aspects of the mechanical design, electrical design, and signal processing. The signal processing section reviews the calculation of instrument attitude and motion in inertial space, develops an algorithm that can use inexpensive lowpower sensors to measure instrument motion, describes the data processing done on all the raw signals, and simulates the inertial processing. The inertial simulations show that when using this inertial algorithm, errors in measuring vorticity and shear resulting from imperfect knowledge of buoy motion, are much smaller than errors resulting from flow disturbance. Readers not interested in inertial processing can skip the signal processing subsections, except the data processing subsection, and still follow the rest of the thesis.

\section{A. MECHANICAL DESIGN OVERVIEW}

In this instrument, circulation around a closed square path is measured, which via Stokes theorem, is the area-integrated vorticity over the surrounded area, equation (2-1).

$$
\begin{gathered}
\iint_{A} \vec{\omega} \cdot \vec{n} d A=\oint_{c} \vec{v} \cdot \overrightarrow{d s}=\Gamma \\
\vec{\omega}=\nabla \times \vec{v}
\end{gathered}
$$

In this equation $\vec{v}$ is velocity, $\vec{\omega}$ is vorticity and $\Gamma$ is circulation. Vorticity is the curl of the velocity field. The $\mathrm{z}$-direction vertical shear of a horizontal $\mathrm{x}$-directed velocity corresponds to a vorticity in the y-direction. A square was chosen for the circulation path to minimize disturbance to circulation in a constant mean flow. A triangle has fewer paths, but as Fig. 2-1 shows, a mean current could produce a wake on one side that would generate a significant measured circulation where one did not exist in the undisturbed flow. The wakes resulting from a symmetrical square of transducers largely cancel out, minimizing the vorticity error due to wakes.

The water velocity in each path around the square is measured acoustically. Differential acoustic travel times on each path are measured by modified Benthic Acoustic 


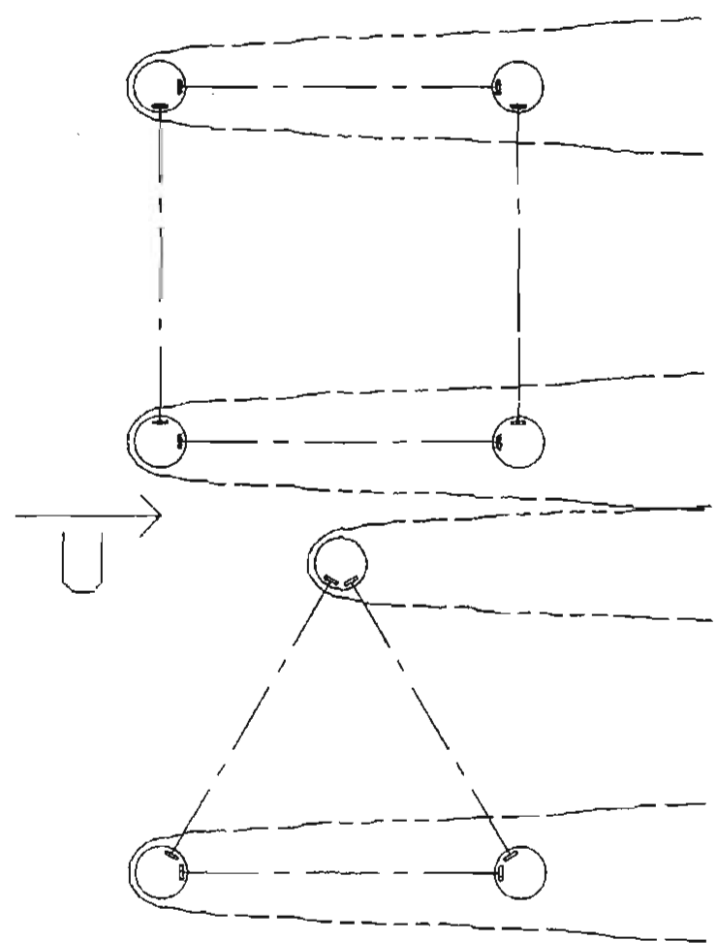

Fig. 2-1 Circulation error from transducer wakes largely cancel out with a square geometry but not with a triangular geometry.

Stress Sensor (BASS) electronics (Williams et al, 1987). The water velocity $v$ parallel to each acoustic path is given by equation (2-2) where $c$ is the speed of sound, $\Delta t$ is the

$$
v=\frac{c^{2} \Delta t}{2 l}+o\left(\frac{v^{2}}{c^{2}}\right)
$$

differential travel time, and $L$ is a single, acoustic-path length making up one side of a square. BASS electronics were chosen to measure velocity because of their speed, accuracy, and low noise; single-pulse time noise is forty picoseconds. The option of using acoustic mirrors and fewer transducers was dismissed due to problems in distinguishing the signal from reflections off the structure.

The velocity of each path around the square is added to give the circulation 
divided by path length (Fig. 2-2). Circulation divided by path length is plotted instead of true circulation or vorticity, because it has the same units as velocity so that cancellation
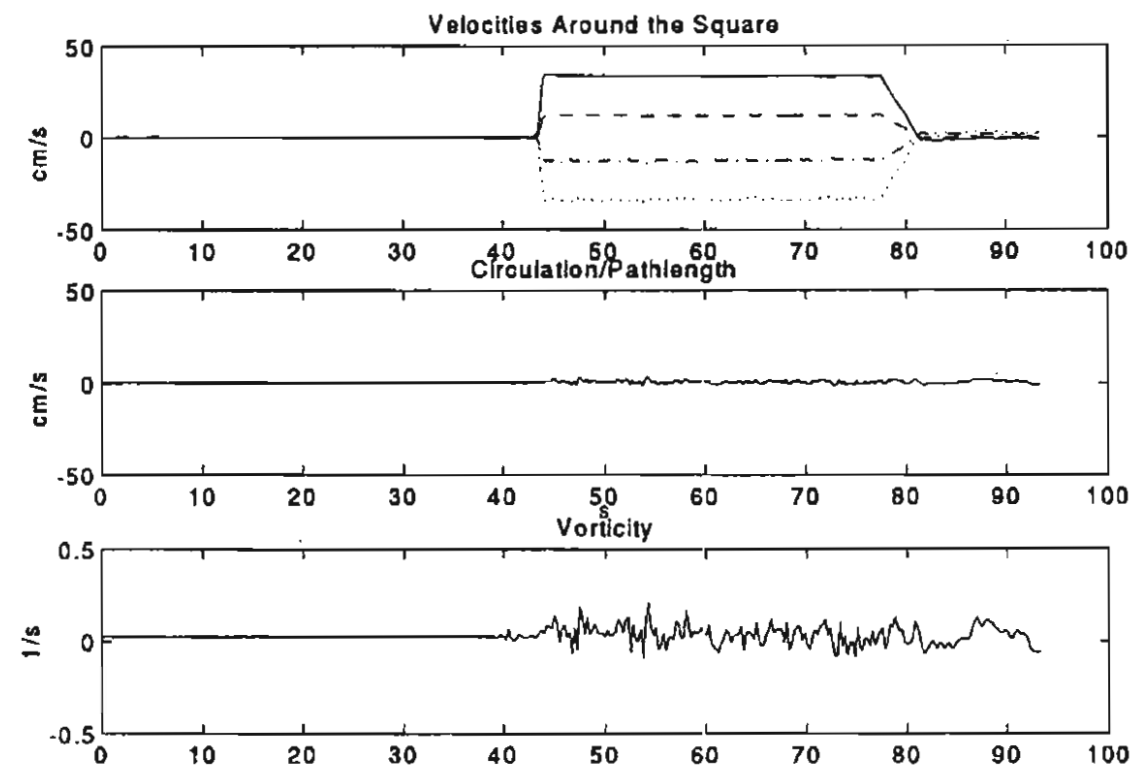

Fig. 2-2. Results from a typical tow tank run showing measured velocity from each acoustic path around the square, circulation divided by path length, and vorticity. This sensor was at an angle to the flow.

of antiparallel velocities can be evaluated. The prototype lab vorticity meter, when oriented with two of the acoustic paths parallel to the flow, (the worst orientation for velocity measurement) measured ten percent less velocity than the undisturbed flow. Parallel paths, however, have the same decrement to within one percent, resulting in small vorticity error.

A three-axis vorticity sensor was designed to minimize wake-related errors when buoy deployed. For an instrument to be deployed on an ocean buoy, all three axes of 
vorticity have to be measured because buoy attitude can change. Buoy motion can then be compensated for, in signal processing, if the buoy's motion is known. A prototype vorticity meter with 15 centimeter acoustic paths was made and is shown in Fig. 2-3. The

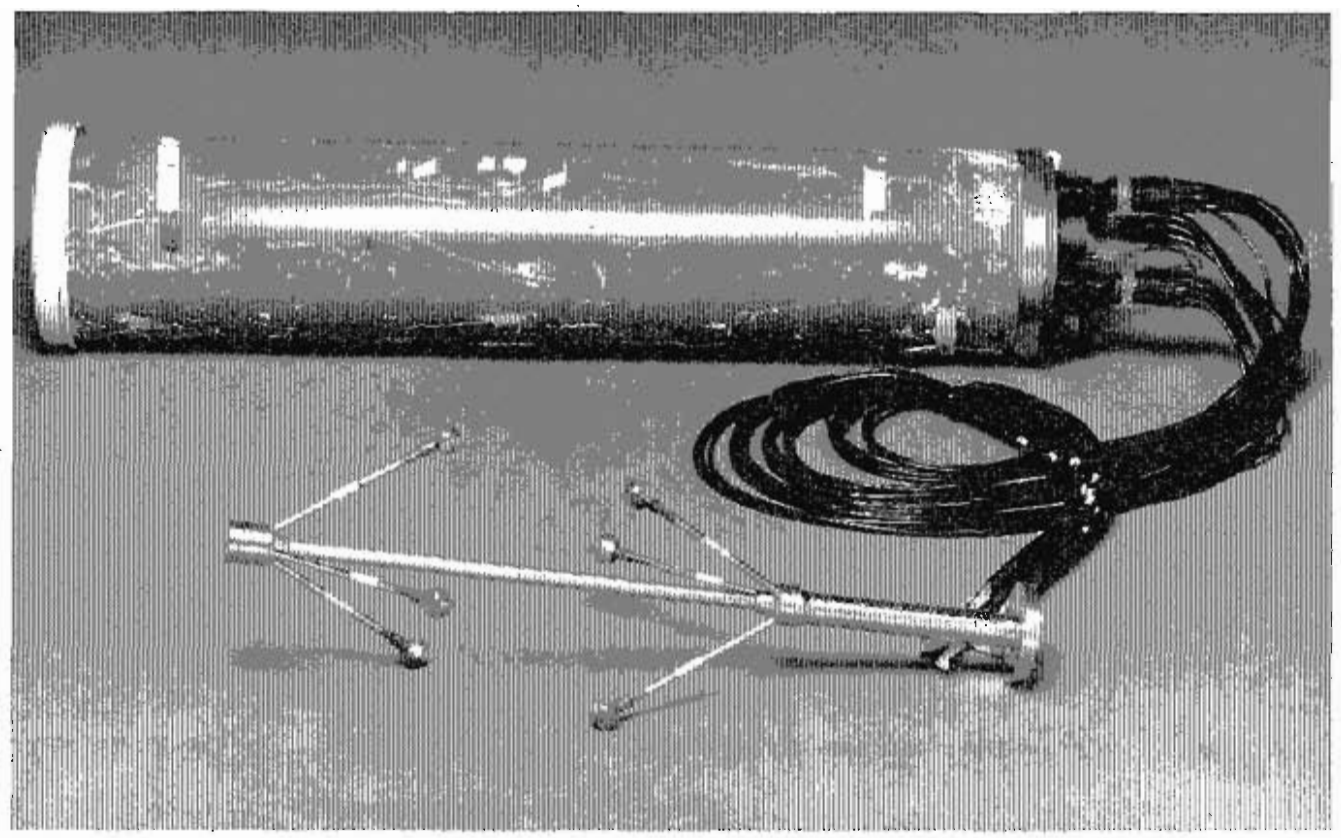

Fig. 2-3. Photo of the prototype three-axis vorticity meter with $15 \mathrm{~cm}$ acoustic paths.

15 centimeter path length was chosen for convenience in the lab and compatibility with existing electronics. Twelve acoustic paths form the edges of a regular octahedron and form three, orthogonal, circulation path squares. Each sphere contains four piezoelectric transducers that form the corner of two circulation path squares. Because a flow sensor mounted on a buoy may measure flow in any direction, but cannot be streamlined in all directions, the design philosophy was to minimize wake asymmetries and errors in circulation. This geometry maximized symmetry and minimized measured circulation flow disturbance. If some of the parts are streamlined, such as the cylinders, they would become lifting surfaces when the flow angle of attack changes and create lift-related circulation, ie., error. Velocity defects symmetric with respect to acoustic circulation paths, do not contribute significantly to error in measured circulation. This prototype sensor was tested for bias in uniform flow in tow tanks and wave rejection in a wave tank 
with the results of these tests justifying our building several larger versions.

The ratio of circulation signal to wake induced noise is expected to increase with longer path length so the ocean deployed instruments were scaled up from the prototype. Two vorticity sensors with 45 centimeter acoustic paths, were built into an inertially instrumented buoy to measure shear Fig. 2-4. A vorticity sensor with a 1.5 meter acoustic

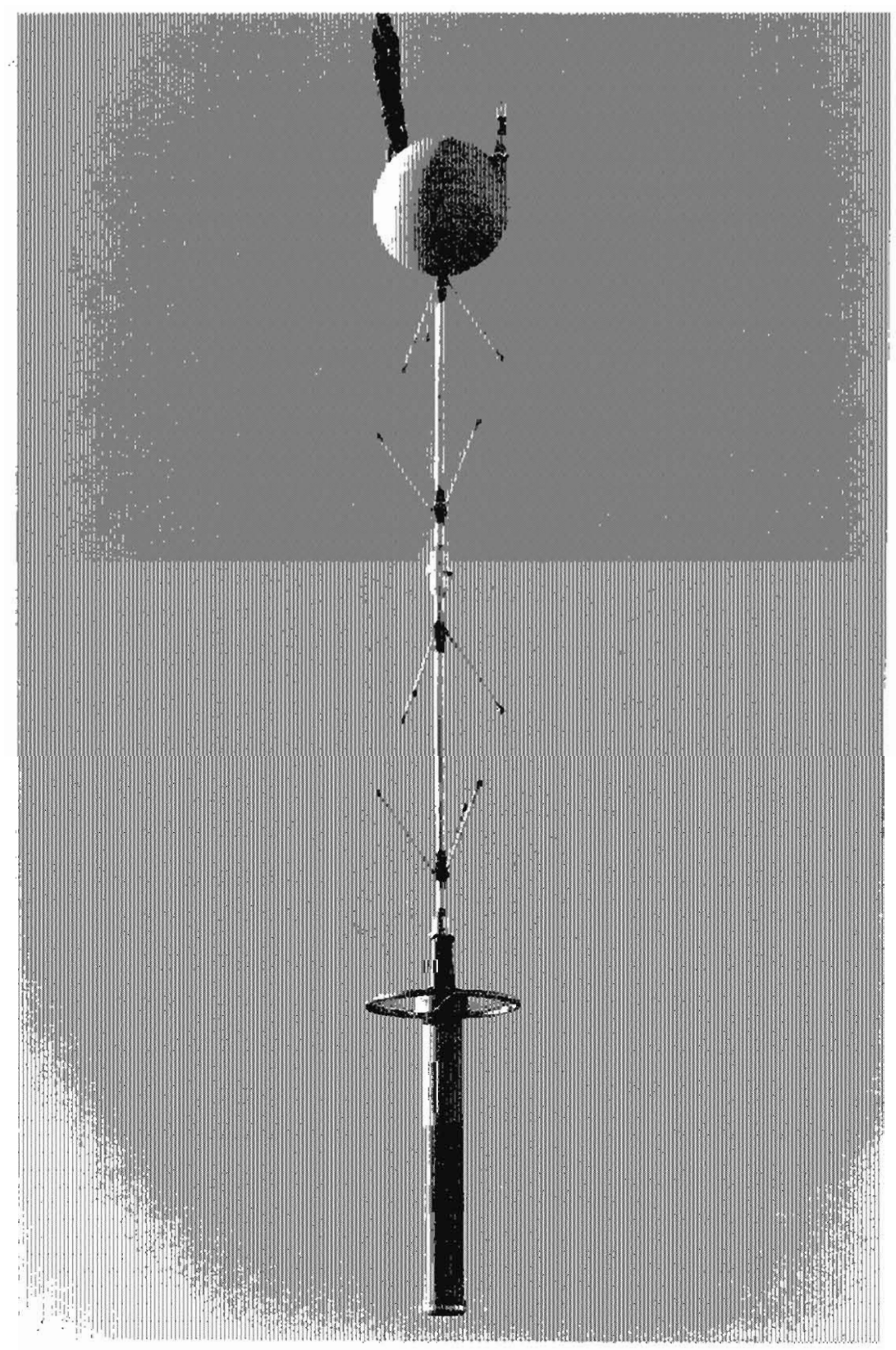

Fig. 2-4. Shear measuring buoy 
path length, was built as its own tripod for measuring the turbulent vorticity and shear in the bottom-boundary layer; this sensor will only be briefly commented on in the performance section where it is used to show spectral rejection of waves during a coastal deployment (Williams, Terray, Thwaites, and Trowbridge, 1994).

The sensor geometry uses a center structural stalk to reduce circulation disturbance as compared to using a braced frame. A braced frame such as one that BASS uses, has a much better strength and stiffness to weight ratio, and can utilize smaller structural members. Its members are then, however, at a radius from the measurement volume center, and any asymmetry between structural member wakes and acoustic paths would create large circulation disturbances. Tube bending strength is proportional to the tube diameter cubed and bending stiffness to the diameter to the fourth power, while drag and consequent wake size is linear to the tube diameter. If one is looking for strength to drag ratio, a nonbraced larger center tube is not at a disadvantage. An additional benefit of this design is that all the wires are strung inside the tubing and not exposed to hydrostatic pressure. Hydrostatic pressure applied to cables squeeze the cables and change the cables' capacitance, which can cause instrument zero drift.

The sensor structure was kept smooth to avoid tripping its boundary layer. The Reynolds number for a two-centimeter tube or sphere at one meter per second velocity is about $2 * 10^{4}$ which is below the laminar-to-turbulent, boundary-layer transition zone. For a sphere, this transition zone Reynolds number ranges from $7 * 10^{4}$ to $5 * 10^{5}$ depending on such variables as surface roughness, ambient turbulence, and structural vibration (Potter and Foss, 1982).

One drawback of this octahedron design is a lack of redundancy. If any of the twelve acoustic paths fails on a buoy deployment, there will be no earth-referenced vorticity without using the questionable assumption of zero correlation between buoy attitude and water motion. This lack of redundancy could only be solved with a substantial increase in flow disturbance. 


\section{VORTEX SHEDDING}

Any bluff body (at these Reynolds numbers) moving with respect to a fluid has oscillatory forces exerted on it by the fluid which are associated with the body's von Karmon vortex street. Acoustic current meters are sensitive to instrument strumming. The largest oscillatory force on a tube is perpendicular to both the tube and the flow direction, at a frequency given by equation (2-3) (Blevins, 1977). In this equation $f_{\mathrm{s}}$ is the

$$
\begin{aligned}
f_{s} & =\frac{S U}{D} \\
S & \approx 0.2
\end{aligned}
$$

frequency of the periodic forcing, $S$ is the Strouhal number, $U$ is the relative velocity, and $D$ is the body diameter. Vibration can have a strong organizing effect on the von Karmon wake, which increases the lateral force exciting the structure, and can cause lock-in of lightly-damped structures. In addition to the large lateral exciting force just described, there is a smaller oscillating force parallel to the flow at twice the frequency given above that can cause lightly damped structures to lock-in at this higher Strouhal number (Crandall, Vigander, and March, 1975). The braised stainless steel structure of the vorticity meter has very little damping, so that strumming has to be considered.

The prototype with the 15-centimeter path, showed no problems with strumming. The first natural frequency of the pod arms was measured to be $123 \mathrm{~Hz}$ and that of the center stalk to be $28 \mathrm{~Hz}$. The pod arms had polyurethane injected into the annulus between the wires and the tube interiors. While this did not effectively add damping, it did keep the cables from rattling around and acted as a pressure block. Smaller structures naturally have higher natural frequencies than larger structures.

The vorticity sensors with the 45 centimeter acoustic paths, were designed with thin pod arms for minimum flow disturbance. The pod arms vibrate at $30 \mathrm{~Hz}$ with a damping ratio of only 0.00038 , measured with the logarithmic decrement method over 
1,800 cycles (Meirovitch, 1975). In a constant flow, the first strumming occurs at $70 \mathrm{~cm} / \mathrm{s}$ and is in the double frequency parallel mode. As mentioned previously, the buoy that these sensors form, was designed to ride with ocean swell. In the open ocean deployments the relative velocity between the sensors and water has never been this large so strumming has not been a problem.

As an additional project, two more 45-centimeter path, vorticity sensors were built to measure bottom-boundary-layer turbulence in high tidal currents. The design changes for these sensors will be discussed as well. The solution we chose was to raise the pod arms' natural frequency and damping by adding pipes that were half as long as the pod arms, screwing them into the center stalk, and filling the annulus between the pod arms and pipe with polyurethane resin. Polyurethanes have high damping and are often used to absorb vibration energy. The resulting natural frequency of the pod arms was doubled and had high damping. These sensors were tested and observed in Vineyard Sound, MA. in a two knot tidal current and showed no signs of strumming. In designing this modification, physical dynamic models were made and tested with polyurethane by itself, fiberglass in epoxy, carbon in epoxy, and fiberglass in polyurethane, in addition to the double tube filled with resin. None of the other damping designs were effective at the lower structural modes of vibration.

\section{MATERIAL SELECTION}

The sensor frames are silver-braised, 316 stainless steel while the instrument case is anodized 6061 aluminum. The silver braise did not corrode in fresh water, but did have to be protected (in this case by polyurethane) in seawater. Other materials considered but not chosen included 316L stainless steel, titanium, and carbon fiber. Titanium and $316 \mathrm{~L}$ stainless steel were not available in the shapes needed, and titanium cannot be braised satisfactorily. Gun-barrel drilling all the tubes in this structure would have been too expensive. The instrument, in general, was designed for "one off" manufacturability with all shapes machinable using standard cutters, and all material available from stock sizes. 
Carbon fiber was not used because of its cost and in this application provided marginal advantages. Carbon fiber structures, when at least vacuum bagged during curing, have better strength-to-weight ratios and much better stiffness-to-weight ratios than metal; however, to achieve this level of performance requires all the excess resin to be squeezed out. This necessitates the molds to be "two and a half" dimensional in that they must have one surface that moves as the excess resin is removed. This reduces design flexibility. High-strength carbon structures have to be carefully designed and laid up because of carbon's brittleness. The primary motive in considering the use of carbon was to increase structural natural frequencies, to push up the minimum velocity of strumming. In air wherein added mass is negligible, carbon fiber structures can be very competitive; but in water the large added mass lowers the natural frequencies to where they would be if the structure was made of metal. The one structural quality of composite structures that is still desirable is their larger structural damping than metal. However, as the strength of a composite material improves, its damping is reduced. Carbon's small additional structural damping did not justify its significantly higher cost.

\section{TRANSDUCERS AND THEIR MOUNTING}

The vorticity meter uses piezoceramic transducers to transmit and receive sound waves at 1.75 MHZ. The piezoceramic used was Transducer Products LTZ-2. Specifications for alignment accuracy can be derived from modeling the transducer as a vibrating piston in an infinite plane wall (Dowling and Ffowcs Williams, 1983). This model gives a pressure at a point given by equation (2-4) where $p^{\prime}$ is the acoustic pressure,

$$
p^{\prime}(\vec{x}, t)=\frac{\rho_{0} i \omega a^{2} U_{o}}{2 R} e^{i \omega\left(t-\frac{R}{c}\right)} \frac{2 J_{1}\left(\frac{\omega a \sin \theta}{c}\right)}{\frac{\omega a \sin \theta}{c}}
$$

$\rho_{0}$ is the density, $\omega$ is the frequency, $a$ is the transducer radius, $U_{0}$ is the velocity of transducer surface, $R$ is the distance to point where the pressure is calculated, $J_{1}$ is the Bessel function of the order one, and $c$ is the speed of sound. The relative beam patterns 
for 0.25 inch $(0.63 \mathrm{~cm})$ and 0.375 inch $(0.953 \mathrm{~cm})$ diameter transducers are shown in Fig. 2-5. The half-power beam width for the 0.375 inch transducers is 2.7 degrees and for the 0.25 inch transducers is 4.1 degrees. These angles correspond to mounting the transducers to the same tolerance of 0.018 inch $(0.46 \mathrm{~mm})$. Because of concern for ganging of errors, the final mounting accuracy specification was 0.01 inches $(0.25 \mathrm{~mm})$. Alignment of the frame was achieved during braising by attaching a jig consisting of steel rods that were screwed in each acoustic path and fitting all the joints loosely. This technique avoided ganging of errors from all the joints.

The transducers were mounted in polyurethane resin on a stainless steel backbone as shown in Fig. 2-6. The polyurethane used was Conap brand EN-4; we chose it because it is acoustically transparent in water. Insulating the transducers from the steel is a spacer

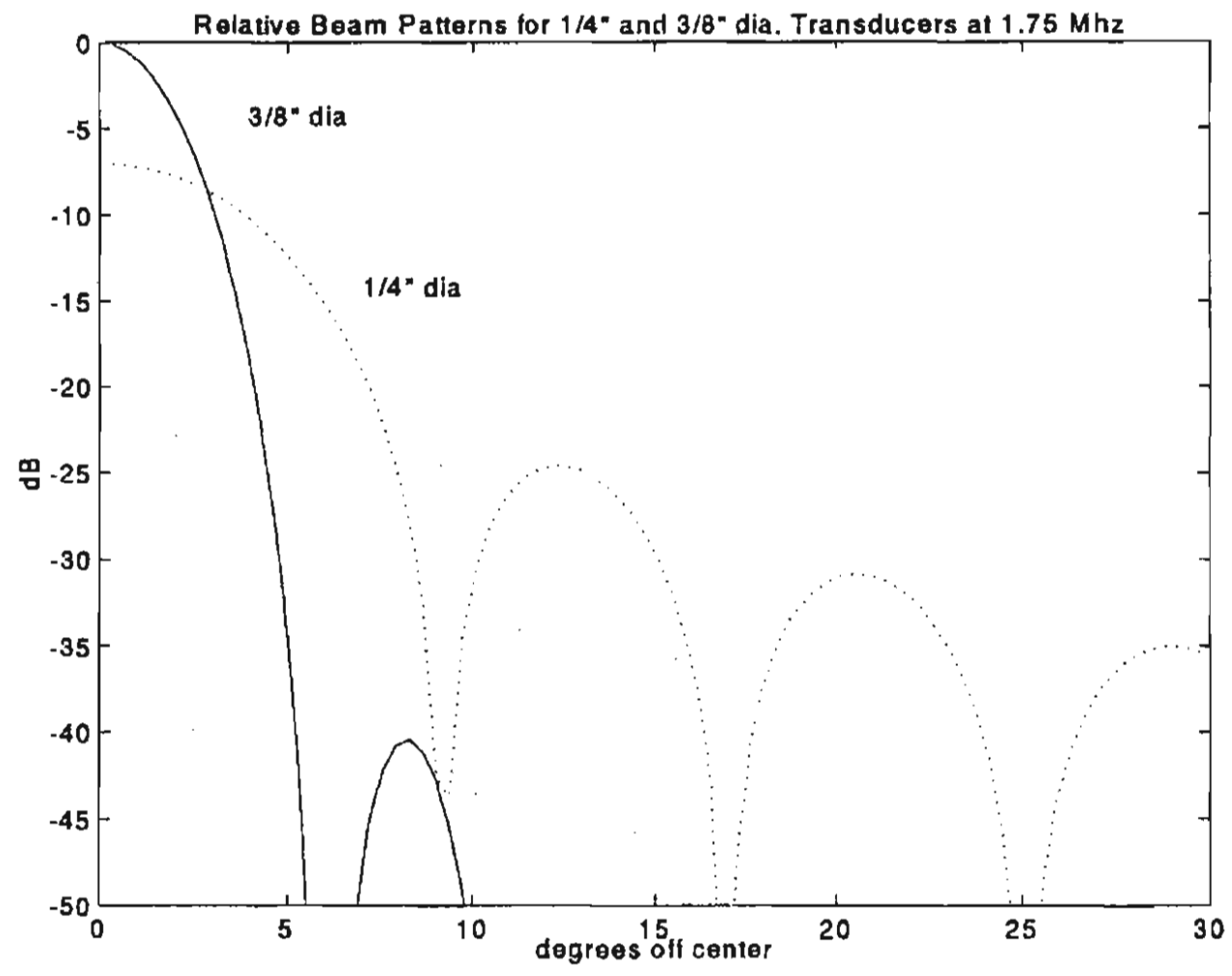

Fig. 2-5. Relative beam patterns for 0.25 inch $(0.635 \mathrm{~cm})$ and 0.375 inch $(0.953 \mathrm{~cm})$ transducers at $1.75 \mathrm{MHZ}$ calculated from eqn (2-4). 
of Dacron in polyurethane, which was found to meet the alignment accuracy needed. The thin Dacron cloth was never fully saturated by the resin, leaving microscopic air bubbles behind the transducers, but this did not cause any measurable problems. The only stainless steel-to-urethane primer found to be effective in seawater was Conap brand AD-6, which has an acid base. If water diffuses through the polyurethane jacket, dissolves the acid residue, and migrates to the transducers; the frit adhering the silver electrodes to the ceramic could be attacked by the acid causing transducer failure. The sensors have been left in freshwater for several months at a time with no degradation, but this potential problem should be monitored on long-term deployments. The entire pod is encapsulated in one-sixteenth of an inch of polyurethane.

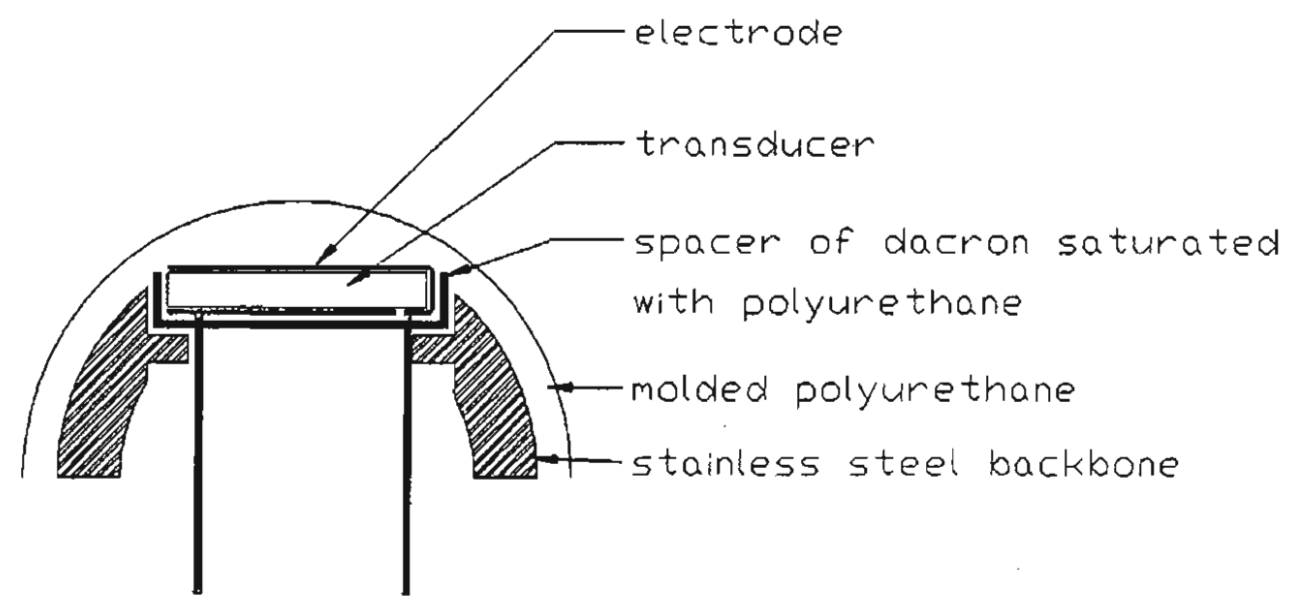

Fig. 2-6. Transducer mounting on stainless steel backbone

Piezoceramic transducers have a high acoustic impedance relative to water, causing a low acoustic transmission coefficient of about 0.04 . Quarter wave plates with an acoustic impedance equal to the geometric mean between that of water and ceramic were considered to improve the transducer effectiveness, but their additional cost of over one hundred dollars extra per transducer for the twenty four transducers per sensor, made 
this option unattractive. Instead, the BASS electronics take advantage of the high transducer $Q$ and transmit fifteen cycles of the carrier wave at the transducer natural frequency. The BASS circuit times off the fifteenth cycle of the receiver transducer, whose energy is coherently summed from the fifteen cycles. This technique largely overcomes the low transducer acoustic match with water.

The minimum pod size was set by transducer considerations. Transducers smaller than 0.25 inch in diameter $(0.64 \mathrm{~cm})$ lose much of their effectiveness due to the solder mass connecting the electrode, thereby detuning that area of the transducer and the depolarized zone underneath the solder. BASS electronics use a low-input-impedance cascode receiver to reduce zero drift from cable capacitance change, but this low-input impedance coupled with the high transducer output impedance requires a strong output signal from the receiving transducer.

The 15 centimeter path prototype vorticity meter used 0.25 inch $(0.64 \mathrm{~cm})$ diameter transducers; these are the smallest available with both wires attached to one side of the transducer. The transducer output gain scales as the diameter to the fourth power, requiring that its transmitter voltage be raised relative to a regular BASS. The prototype also used EN-4 polyurethane as a pressure block. This is not a hard polyurethane and could cold flow on a long, deep deployment. These pods ended up having a 1.0 inch (2.54 $\mathrm{cm}$ ) outer diameter.

The 45 centimeter path vorticity sensors used 0.375 inch $(0.953 \mathrm{~cm})$ diameter transducers to avoid excessive transmitter voltages. The acoustic energy from the receiver transducer scales as one over the path length squared. The electronics of this instrument used a three-to-one turns ratio in the toroidal core transformers that power the transducers (Williams et al, 1987). In these sensors, thermistors (Yellow Springs Instrument Co. 44030) were mounted inside the pod arm tubes at a distance of 2 inches $(5 \mathrm{~cm})$ from the pods on each side of each sensor. The thermistors measure temperature and temperature stratification. For greater depth capability, the transducer pods use glass-filled epoxy 
pressure blocks. These pods have a 1.25 inch $(3.17 \mathrm{~cm})$ outer diameter.

The 1.5 meter path vorticity meter briefly mentioned uses 0.75 inch $(1.9 \mathrm{~cm})$ diameter transducers and operates at the lower frequency of $875 \mathrm{KHz}$ to keep transducer alignment from becoming too difficult; it is difficult to align objects to less than one degree.

\section{BUOY DESIGN}

A shear measuring buoy was built of two 45-centimeter path, vorticity sensors, their electronics, an inertial measurement unit, and a float with recovery gear, Fig. 2-4. The buoy was designed to minimize flow disturbance in the measurement volumes by moving with ocean swell, minimize surface expression (the float), minimize sensor cylinder size, and locating the electronics package well below the measurement volumes. Measurement volumes are centered at 0.83 meters and 2.45 meters below the water surface, and the total buoy height is 5.06 meters. The buoy has a strapdown, inertial measurement unit to measure buoy motion and remove it from relative flow, and to rotate the measurements into an Earth reference system. The inertial measurement unit is described in the electrical design section and the inertial processing is described in the signal processing section.

The buoy is modular and is bolted together with interchangeable sensors. A bumper of rolled, welded stainless steel tubing is mounted on the instrument case so that the buoy can be laid on its side on a flat surface between this bumper and the top spherical float, and not bend the pod arms. The top float is made of expanded PVC foam with a fiberglass skin. This system is light, relatively incompressible, failsafe, shapable, and corrosion free.

The buoy is deployed freely, drifting with the currents, requiring reliable recovery aids. The recovery systems include an ARGOS transmitter that transmits to a satellite which measures and relays buoy location, a strobe light, a VHF radio transmitter, and 
when deployed below the ocean surface, an acoustic transponder that is compatible with a diver-operable, subsea direction finder.

To measure internal wave shear in the thermocline, the buoy can be deployed using an elastic tether between the top float and the rest of the instrument, Fig. 2-7. The drifter uses a bungy cord strung inside a hollow sleeve rope that is slack, to isolate float heave from the instrument. The chain on the float bottom and poly fishnet floats at the instrument's top keep tension on the elastic tether below two kilograms, allowing a lowspring-rate soft bungy to be used. Observations of the instrument during dives showed very effective heave suppression.

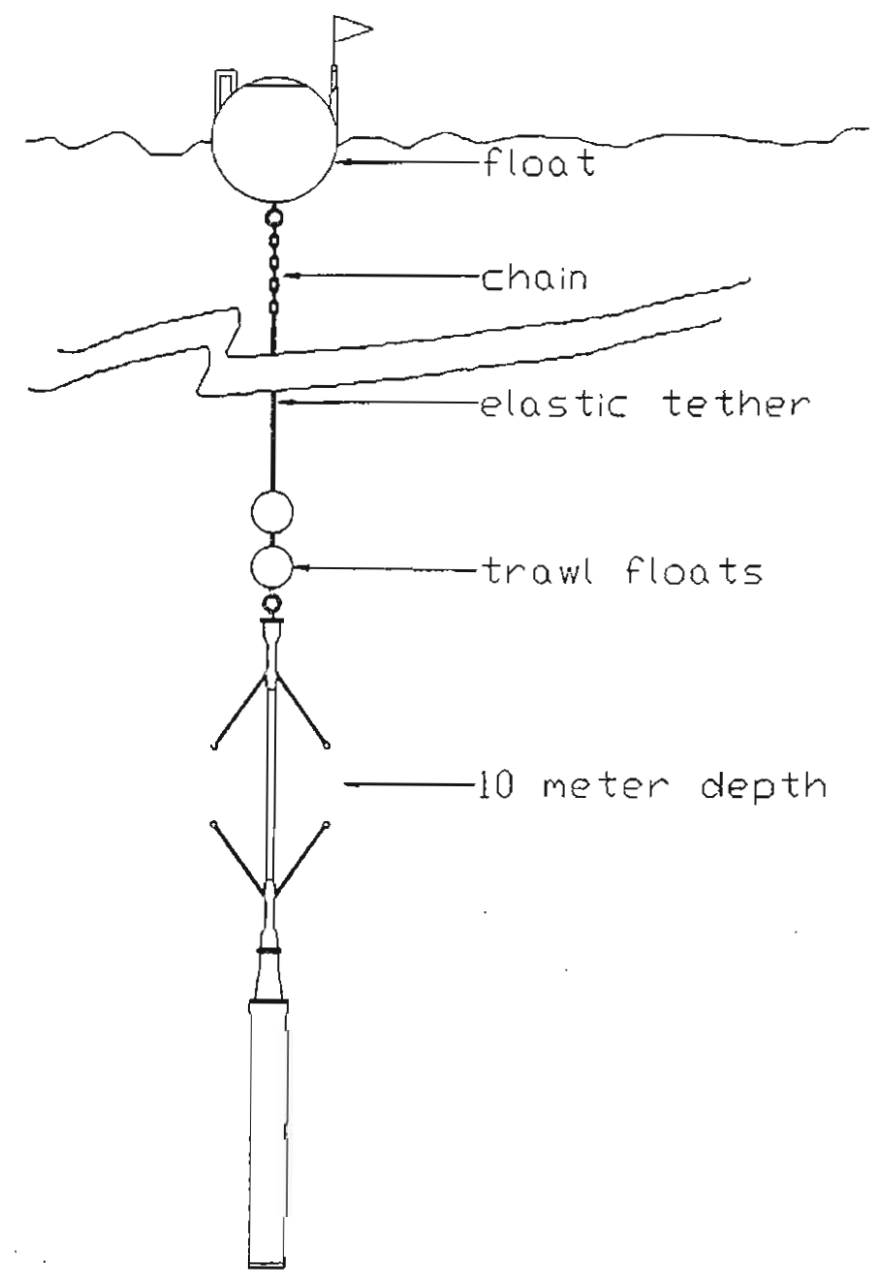

Fig. 2-7. Acoustic vorticity meter configured as a thermocline shear measuring drifter. 


\section{B. ELECTRICAL DESIGN}

\section{ELECTRICAL OVERVIEW}

Modified BASS electronics (Williams et al, 1987) form the heart of the vorticity meter. The physical electronics, inertial measurement unit and data logger are shown in Fig. 2-8. A block diagram of the electrical system is shown in Fig. 2-9. The BASS

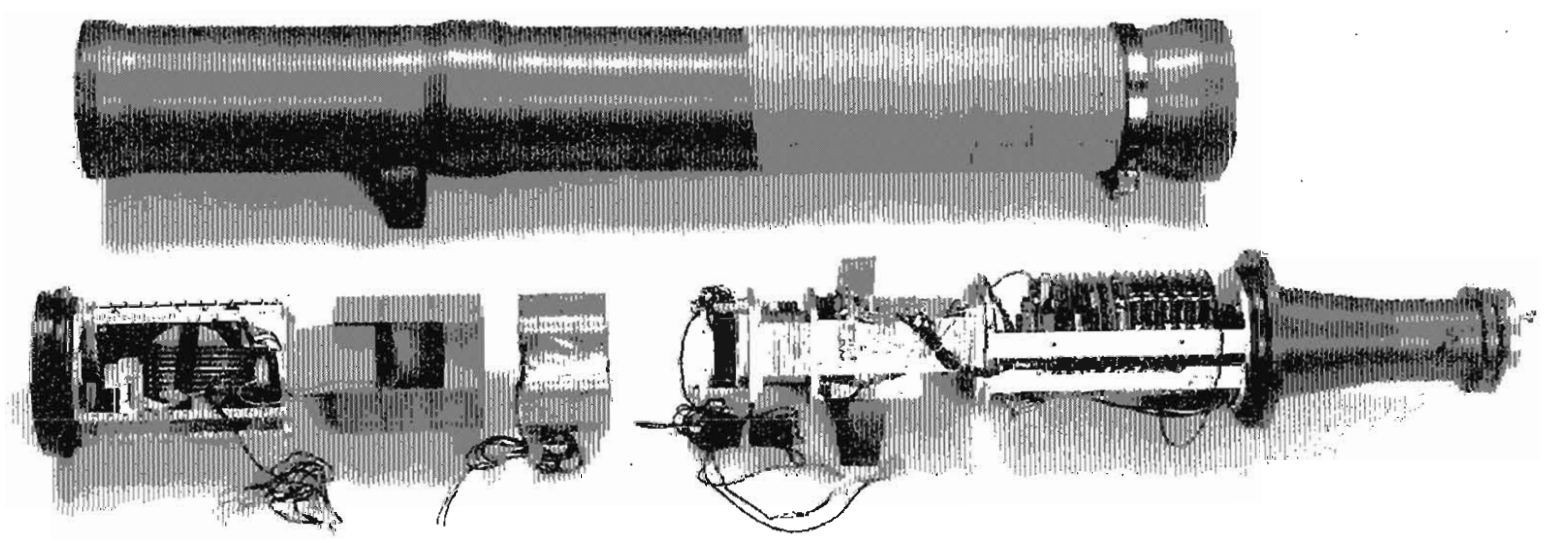

Fig. 2-8. Photo of electronics on right, inertial measurement unit in middle, battery, and data logger at left with the instrument case above.

electronics are controlled by a Tattletale 5 computer made by Onset computers, Pocasset, MA.. The Tattletale 5 computer directs each pair of acoustic transducers to measure water velocity along their acoustic path, polls all the analog inputs from the: thermistors, accelerometers, rate gyros, and magnetometers; and sends this data serially to a data logger. The data logger is a Tattletale 6 computer that records the data on a hard disk drive after buffering the data in semiconductor memory. This "buffering to memory" is done to save power; the hard disk is only spun up and written to when the memory is full. The analog signal conditioning done to the inertial sensors' signals is scaling voltages and 
BASS

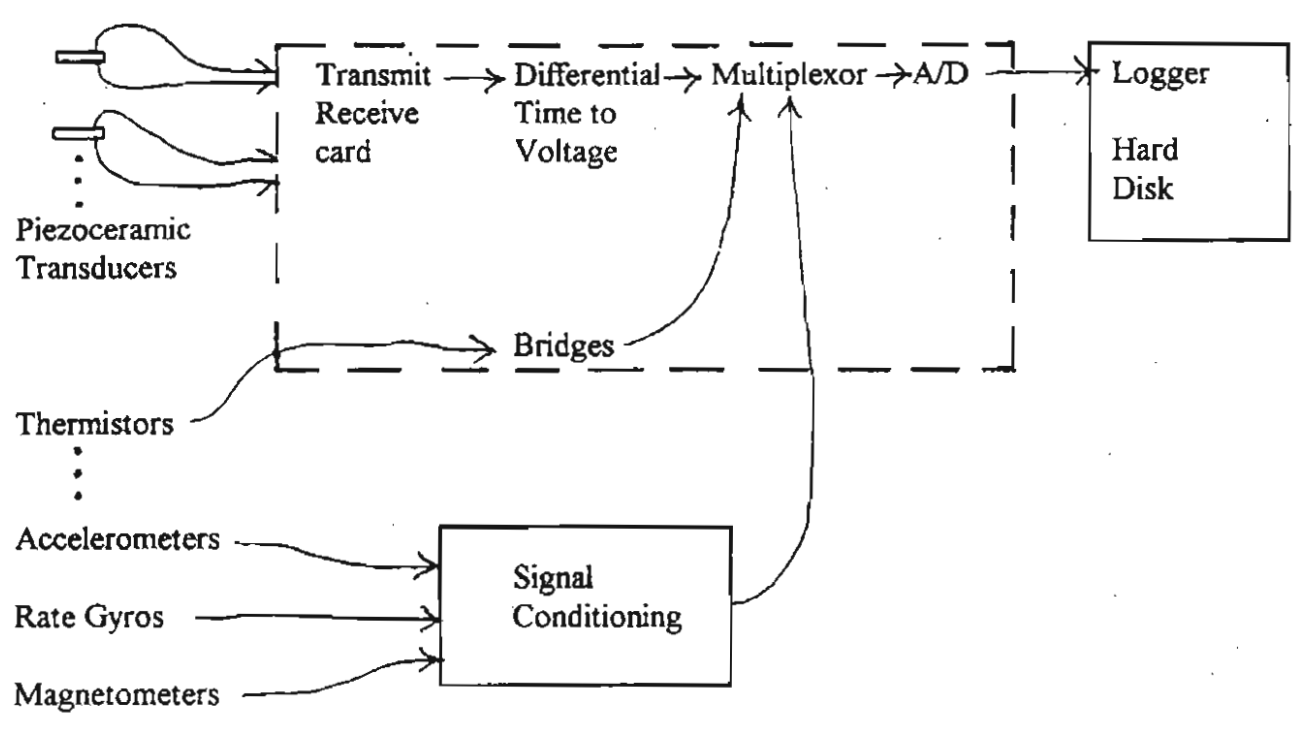

Fig. 2-9. Block diagram of electrical system

low-pass, anti-alias filtering. Each of the recovery devices: the ARGOS transmitter, VHF transmitter, and strobe, has its own battery for redundancy. The recovery devices are not shown in this diagram.

One problem with the data logger system is the high current required to spin up the hard disk before a software timeout is reached. The main batteries of this instrument are alkaline which have a high output impedance when cold, and are unable to start the hard disk below a certain temperature. To resolve this problem, a lead acid gel cell with lower output impedance at low temperature, is used as a capacitor to start the disk. This gel cell is then recharged by the alkaline main batteries between disk writes.

The longer acoustic path length required modifying the transmit voltage and timing of the BASS electronics. To increase the transmit voltage, the transformer cores that drive the acoustic transducers were wound with a three-to-one ratio instead of the one-to- 
one ratio in a regular BASS. The fast timing to control the acoustic velocity measurement is done in analog on the timing and burst generator card to save power. The instrument transmits fifteen cycles at $1.75 \mathrm{MHz}$. for $8.57 \mu \mathrm{s}$. The receiver is turned on $282.5 \mu \mathrm{s}$ after the start of the transmit; this corresponds to the fastest measurable sound speed of 1590 $\mathrm{m} / \mathrm{s}$. The receiver effectively turns off $344.5 \mu \mathrm{s}$ after the transmit start; this corresponds to the slowest measurable sound speed of $1370 \mathrm{~m} / \mathrm{s}$. The acoustic path velocity is then remeasured $383.5 \mu$ s later with the electronics reversed to cancel out electronic drift. The measurement cycle consists of a time stamp of four bytes, 24 forward and reverse velocity measurements that take $18.4 \mathrm{~ms}, 13$ analog measurements of the thermistors and inertial sensors, and then sending the eighty byte measurement sequence at 9600 baud to the logger, taking $83 \mathrm{~ms}$. The whole measurement sequence is repeated every $150 \mathrm{~ms}$.

The data format is shown in Table 2-1. All the data is transmitted and stored in unsigned binary integers except the velocity measurements which are in two's complement.

EE DD

$\{1\}\{1\}$

$\{2\}\{2\}$

2\}

$\{2\} \quad\{2\}$

\{2\} $\{2\}$

$\{2\} \quad\{2\}$

$\begin{array}{ll}\{2\} & \{2\} \\ \{2\} & \{2\}\end{array}$

$\{2\} \quad\{2\}$

$\{2\} \quad\{2\}$

$\{2\} \quad\{2\}$

$\{2\} \cdot\{2\}$

\{2\} $\{2\}$

$\{1\}$

$\{2\}$

$\{2\}$

\{2\}

$\{2\}$

$\{2\}$

$\{2\}$

\{2\}

\{2\}

$\{2\}$
$\%$ time $\%$ podl $\%$ pod2 \% pod3 \%

$\{2\} \quad$ pod4 \%

\{2\} pod5 \%

\{2\} pod6 \%

\{2\} temp \% imul \% imu2 \% imu3 \% hexadecimal record header time stamp hr:min:s:counter four acoustically measured velocities making up a circulation square

thermistor output accelerometer rate gyro magnetometer

Table 2-1. Vorticity meter data format. The numbers in brackets indicate the length of each variable in bytes 


\section{INERTIAL MEASUREMENT UNIT}

A strapdown inertial measurement unit was built into the shear measuring buoy to measure buoy motion allowing the motion to be removed, in processing, from flow measurements. The Inertial Processing Background section shows that it is necessary to measure and compensate for buoy motion even if only time average measurements from a buoy are desired. A strapdown inertial measurement unit was chosen over a gimballed inertial measurement unit to save power and size. A gimballed inertial measurement unit, consisting of accelerometers on a gyro-stabilized platform that does not rotate with respect to inertial space, is more accurate than a strapdown system consisting of the accelerometers, rate gyros and magnetometers mounted "strapped down" to the buoy (VanBronkhorst, 1978). Gimballed systems are expensive, delicate, and consume much power; whereas strapdown systems are inexpensive, more robust mechanically, and consume less power. Strapdown systems do, however, require more computation. Gimballed systems use rate gyros as nulling sensors, do not expose the rate gyros to the angular rates of the buoy, and therefore can measure angular rates more accurately. Strapdown systems, on the other hand, expose their rate gyros to the full angular rates of the buoy, requiring rate gyro accuracy and linearity over a wide range. Gimballed systems are able to cancel out some error terms, such as some small accelerometer misalignment, that strapdown systems can not cancel out (Schmidt, 1978). Some wavebuoys use a lowpower version of a gimballed system by floating a large sphere with accelerometers, in oil, and limiting the system's righting response to frequencies lower than the wind-wave spectrum. These wavebuoy systems are however, large and heavy. The shear measuring buoy uses a strapdown inertial measurement unit to save power (battery weight, size, and flow disturbance), to avoid the bulk and weight of some of the wavebuoy inertial measurement platforms, and to save money.

The strapdown inertial measurement unit consists of a three-axis accelerometer, three single-axis rate gyros, and a three-axis magnetometer. These sensors and some of their specifications are listed in Table 2-2. It is worthwhile to note that these are not inertial-navigation-grade sensors. For example, good inertial grade rate gyros are $10^{6}$ 
Three-axis accelerometer

Columbia Triaxial Accelerometer

model SA-307 HPTV

$$
\begin{array}{ll}
X & -1 \text { to }+1 G \\
Y & -1 \text { to }+1 G \\
Z & 0 \text { to }+2 G
\end{array}
$$

case alignment $+/-0.5^{\circ}$

Three single-axis rate gyros Systron Donner Gyrochip Angular Rate Sensor $\mathrm{X}, \mathrm{Y}$, and $\mathrm{Z} \quad-50$ to $+50 \mathrm{deg} / \mathrm{s}$

$$
\begin{aligned}
& \text { bandwidth }>60 \mathrm{~Hz} \text {. } \\
& \text { scale calibration } 1 \%
\end{aligned}
$$$$
\text { linearity }<0.05 \% \text { of full scale }
$$

input power noise requirement $<0.01 \mathrm{v} \mathrm{rms}$ and

$<0.001 \mathrm{v}$ ms at $8.7 \mathrm{Khz} .+/-500 \mathrm{~Hz}$.

Three-axis magnetometer Develco model 9200 fluxgate magnetometer

$\mathrm{X}, \mathrm{Y}$, and $\mathrm{Z} \quad-600$ to +600 mGauss

$$
\begin{gathered}
\text { alignment }+/-1^{\circ} \\
\text { output ripple } 0.4 \% \text { of full scale } \\
\text { temp stability }<3 \% \text { from } 0^{\circ} \mathrm{C} \text { to } 60^{\circ} \mathrm{C} \text { sensitivity } \\
<1 \% \text { zero } \\
\text { linearity }+/-0.5 \% \text { of full scale }
\end{gathered}
$$

Table 2-2. Description of the inertial measurement unit sensors

times more accurate than the low-power rate gyros used in the vorticity meter. The alignment of the sensors used, inside their sensor cages, is only good to about one degree. Techniques such as Schuler tuning are not useful when the rate gyro noise, even when averaged over ten minutes, and drift are ten times the earth's rotation rate. However, with appropriate processing, as is shown in the Inertial Processing section, these sensors are adequate for this application.

The inertial measurement system requirements will now be specified. Errors in estimated buoy attitude and motion should not cause more than five percent error in flow measurements. This translates to an angle error specification of three degrees. Angle rates in sensor coordinates should be better than one percent to compensate for buoy motion. The Inertial Processing Background section shows that the heading specification 
of three degrees actually requires pitch and roll error be less than one degree. The inertial system should be self-aligning in a seaway, because boats that the buoy will be launched from do not have three-axis inertial reference for a conventional system alignment. Errors from coning motions should not be significant. The Inertial Processing Background section discusses coning motions and their importance. While these specifications seem lax by inertial navigation standards, they are adequate for removing buoy motion from the buoy measurements of vorticity. With the sensor specifications given, it would not be easy to do significantly better.

\section{CALIBRATION OF THE INERTIAL MEASUREMENT UNIT}

All calibrations of the inertial sensors were performed with the sensors inside the buoy instrument case, with all batteries, the same electronics, and $\mathrm{A} / \mathrm{D}$ converter that were used when the instrument was deployed at sea. The rate gyros were calibrated by rotating the instrument case in a rotating welding fixture and on a rotating table, at different speeds, and around each axis. The accelerometers and magnetometers were calibrated statically by holding the case at different roll, pitch, and heading angles in the middle of an open field. The magnetometer was calibrated in an open field to avoid the magnetic anomalies in the laboratory. The angular positions were measured on a piece of leveled, one-inch plywood for stability; rested on measured wedges and were calibrated with respect to a single axis magnetometer and to a level. In all these calibrations, the averaging time for each data point was greater than five minutes to ensure reliable statistics. The calibration results are shown in Figs. 2-10 and show good linearity of the sensors, except at the very ends of their measurement limits. The noise from all except one sensor channel was white to the background levels of vibration in the Bigelow building basement. The $\mathrm{Y}$ channel of the rate gyro however, had a 17 second oscillation that corresponds to a 0.5 degree rocking motion (Fig. 2-11). The cause of the noise is unknown, but is within the manufacturer's sensor specification. The standard deviations of the sensor noise are listed in Table 2-3. 

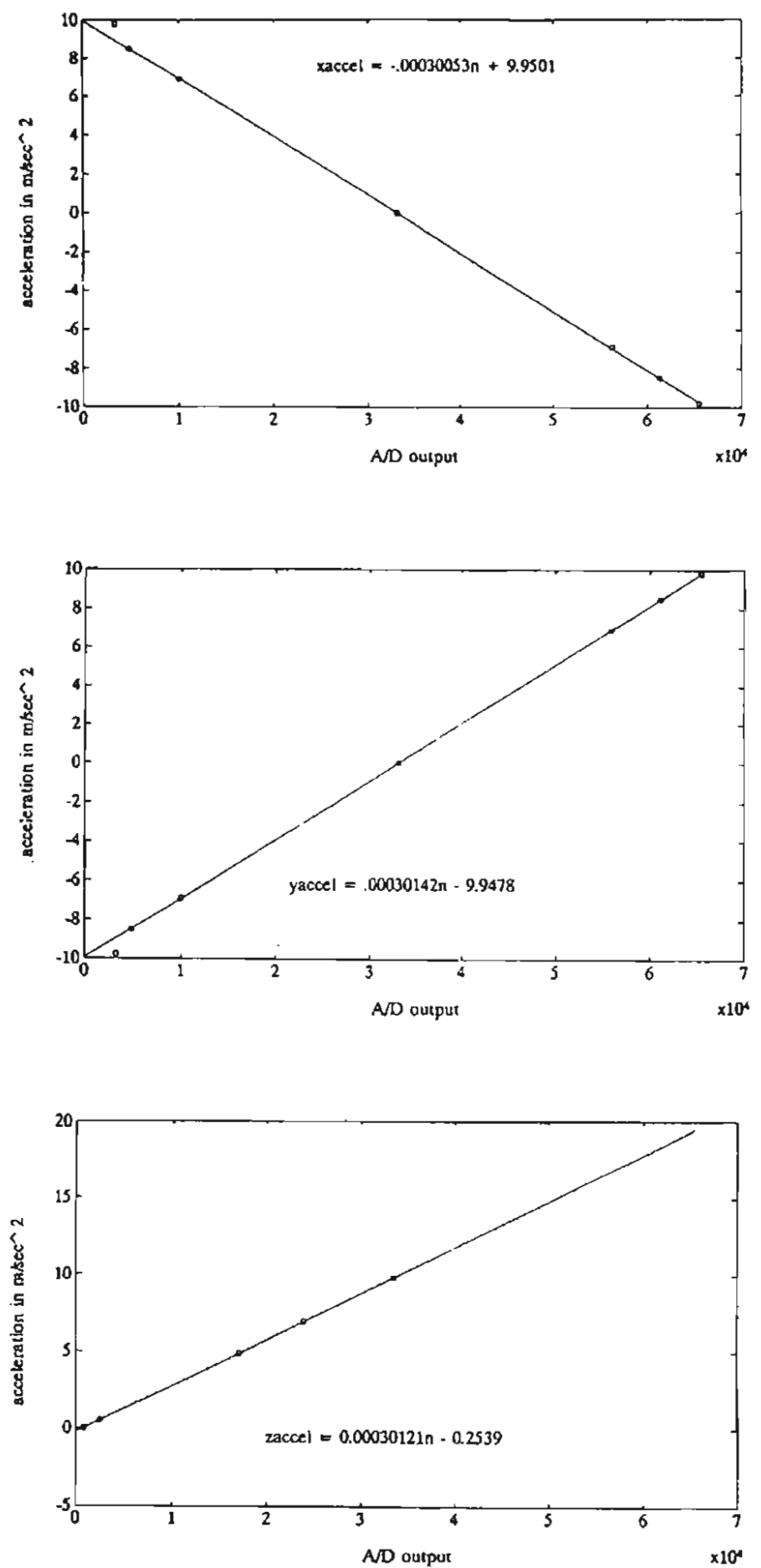

Fig. 2-10 a. Calibration of accelerometers. The top, middle and lower graphs are the calibrations of the $\mathrm{x}$-axis, $\mathrm{y}$-axis and $\mathrm{z}$-axis accelerometers. 

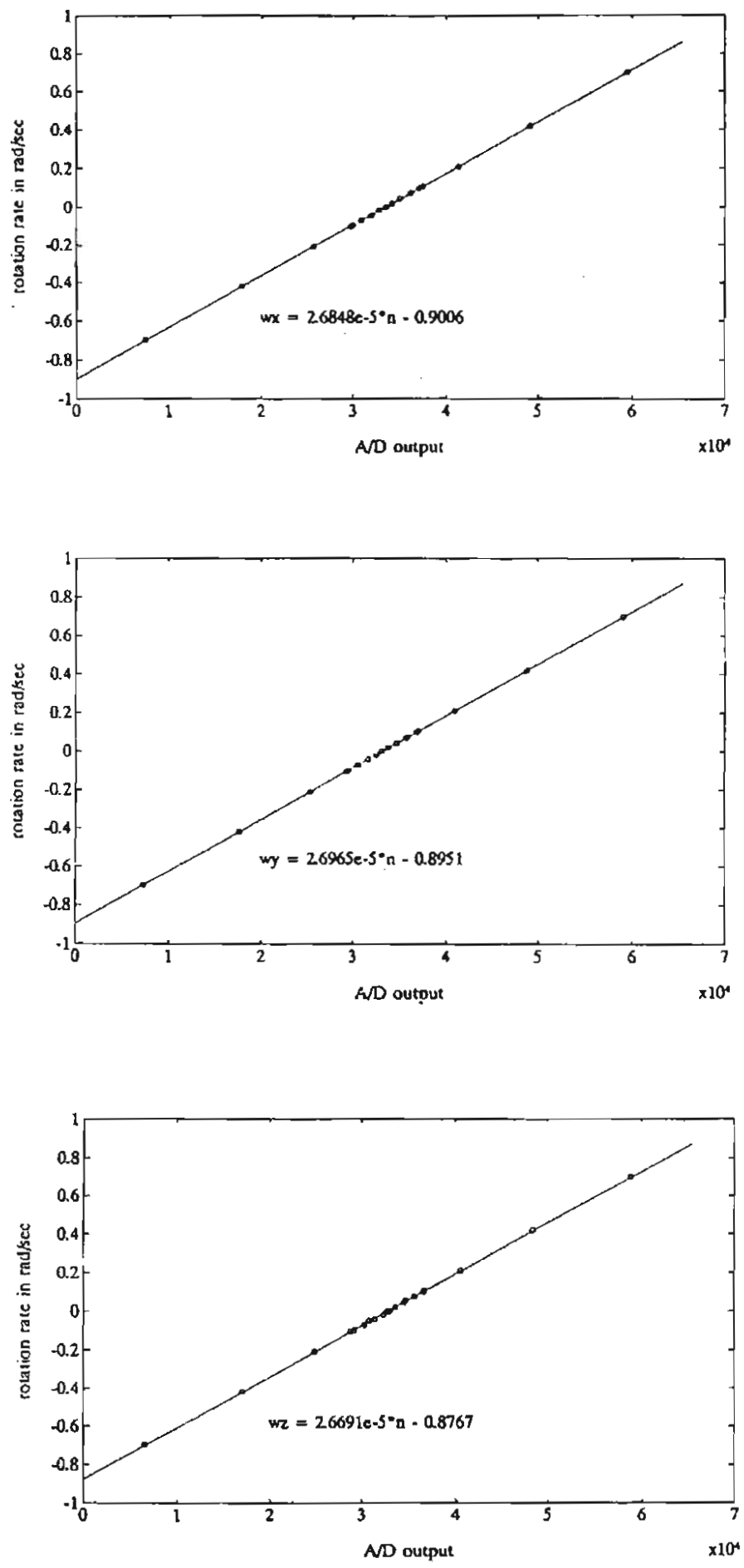

Fig. 2-10 b. Calibration of Rate Gyros. The top, middle, and lower graphs are the calibrations of the $\mathrm{x}$-axis, $\mathrm{y}$-axis, and $\mathrm{z}$-axis rate gyros. 

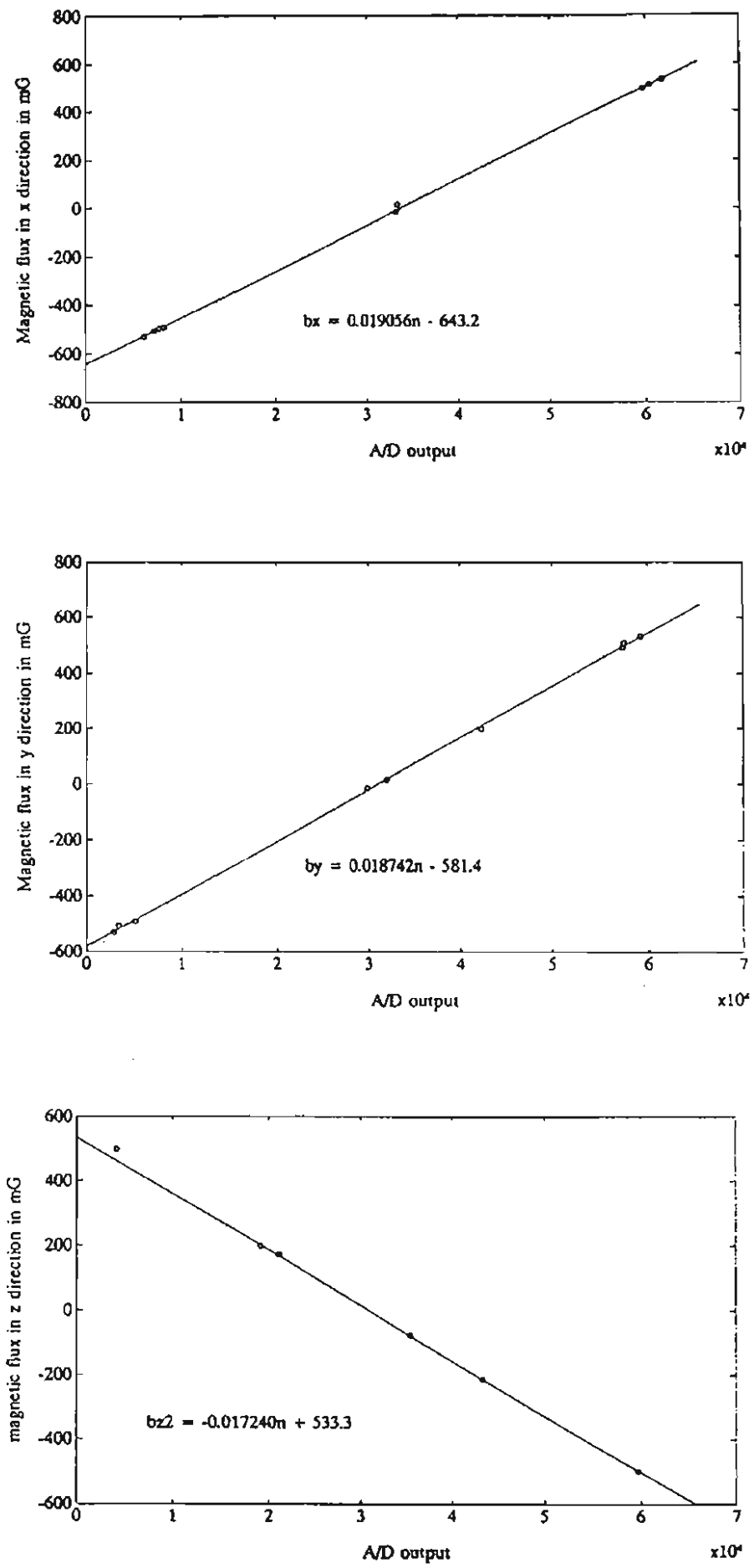

Fig. 2-10 c. Calibration of Magnetometers. The top, middle, and lower graphs are the calibrations of the $\mathrm{x}$-axis, $\mathrm{y}$-axis, and $\mathrm{z}$-axis magnetometer channels. 


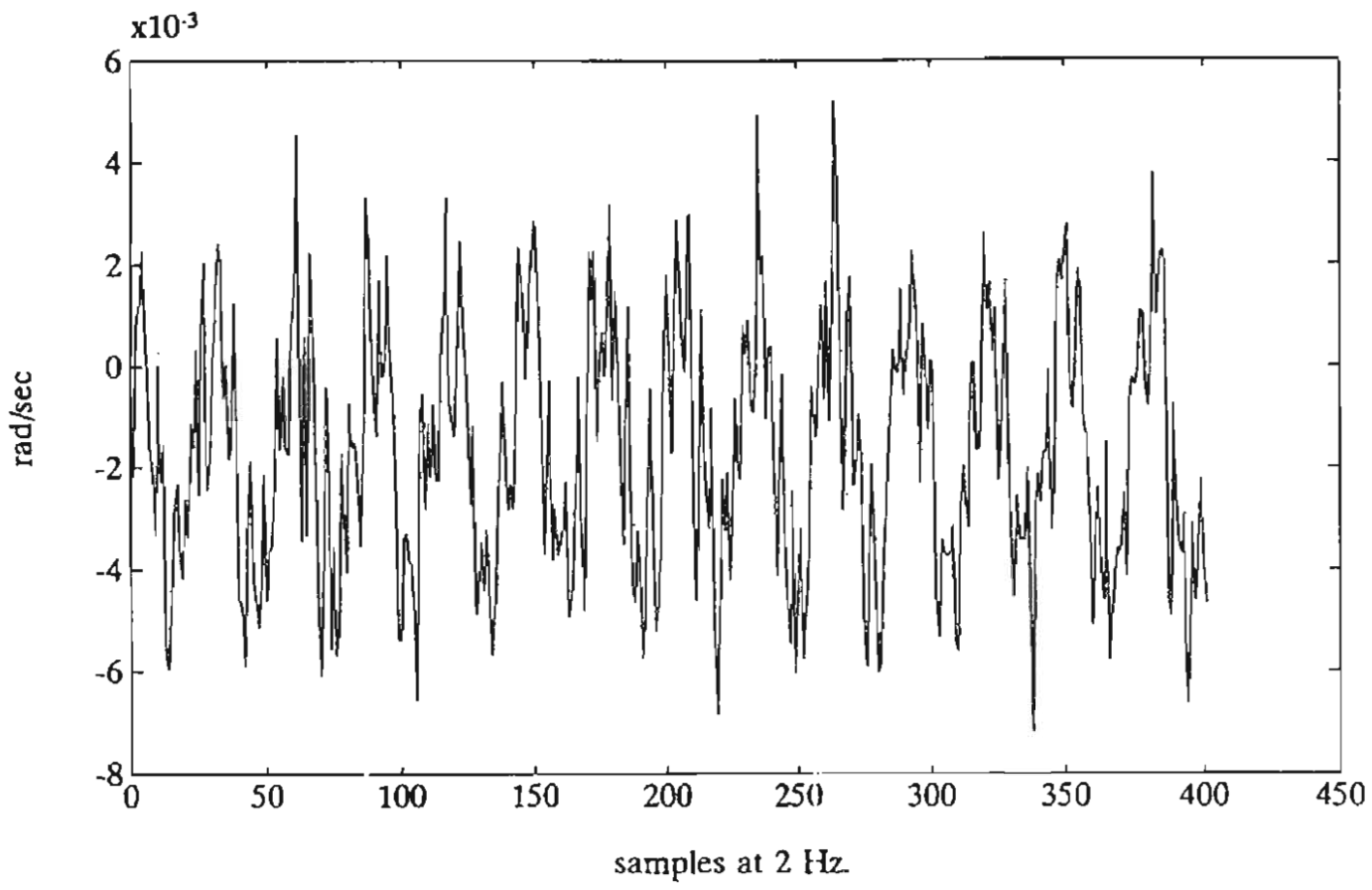

Fig. 2-11. Sample of y-axis rate gyro output noise while instrument was still.

$\begin{array}{llll}\text { SENSOR } & \text { X } & \text { AXES } & \\ & & \mathrm{Y} & \mathrm{Z} \\ \text { accelerometer }\left(\mathrm{m} / \mathrm{s}^{2}\right) & 0.013 & 0.012 & 0.014 \\ \text { rate gyro }(\mathrm{rad} / \mathrm{s}) & 0.0014 & 0.0025 & 0.0015 \\ \text { magnetometer }(\mathrm{mG}) & 0.77 & & 0.74\end{array}$

Table 2-3. Standard deviation of the inertial sensor noise 


\section{C. SIGNAL PROCESSING}

This section reviews the kinematics of rotation, develops an algorithm to calculate buoy motion, describes the total data processing, and simulates this inertial signal processing to estimate its accuracy.

\section{INERTIAL PROCESSING BACKGROUND}

\section{COORDINATE TRANSFORMATIONS}

This subsection reviews the kinematics of rotation and introduces some of the mathematical conventions used in this thesis. The rotation of coordinate system xyz into reference frame $x^{\prime} y^{\prime} z$ ' is shown in Fig 2-12. The transformation of vector coordinates from one reference frame to the other can be described by a matrix multiplication (2-5)

$$
\left\{\begin{array}{l}
x^{\prime} \\
y^{\prime} \\
z^{\prime}
\end{array}\right\}=[A]\left\{\begin{array}{l}
x \\
y \\
z
\end{array}\right\}=\left[\begin{array}{lll}
A_{x^{\prime} x} & A_{x^{\prime} y} & A_{x^{\prime} z} \\
A_{y^{\prime} x} & A_{y^{\prime} y} & A_{y^{\prime} z} \\
A_{z^{\prime} x} & A_{z^{\prime} y} & A_{z^{\prime} z}
\end{array}\right]\left\{\begin{array}{l}
x \\
y \\
z
\end{array}\right\}
$$

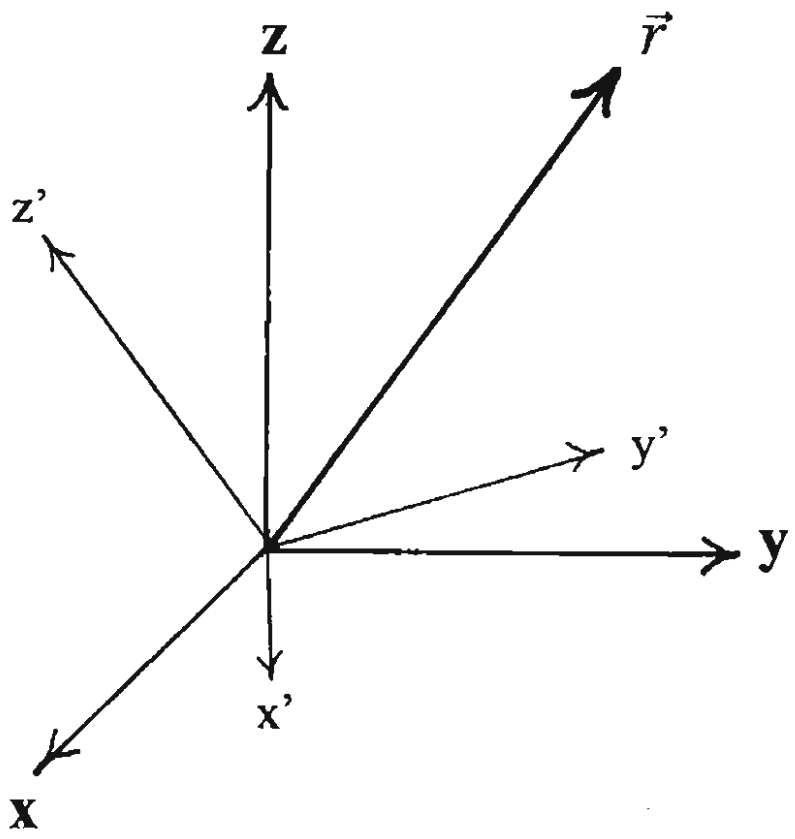

Fig. 2-12. Rotation of coordinate system xyz to $x^{\prime} y^{\prime} z^{\prime}$ 
(Crandall et $\mathrm{al}, 1968$ ). In this equation the vector components $\mathrm{x}, \mathrm{y}, \mathrm{z}$ are the coordinates of vector $\vec{r}$ in the xyz frame and the primed $x^{\prime} y^{\prime} z^{\prime}$ vector components are the coordinates of the vector in the primed frame. This rotational-transformation matrix $A$ is made of direction cosines $(2-6)$. The rotation matrix has six-fold redundancy in direction cosines

$$
A_{x^{\prime} y}=\vec{u}_{x^{\prime}} \cdot \vec{y}_{y}
$$

as there are six, independent relations among the nine elements. Some properties of the matrix are that its inverse is its transpose and its determinant is plus one (2-7). The same

$$
\begin{aligned}
{[A]^{-1} } & =[A]^{\prime} \\
|A| & =+1
\end{aligned}
$$

rotation of the vector $\vec{r}$ referenced in a constant reference frame can be described by the same equation (2-5) but using the inverse of $A$. Successive rotations are not commutative; a rotation $\mathrm{A}$ about the $\mathrm{x}$ axis followed by a rotation $\mathrm{B}$ about the $\mathrm{y}$ axis does not in general equal the rotation $\mathrm{B}$ about the $\mathrm{y}$ axis followed by the rotation $\mathrm{A}$ about the $\mathrm{x}$ axis. Successive rotations can be described as matrix multiplication of the individual rotation matrices, and matrix multiplications are not commutative (2-8). Because of the

$$
[A] *[B] \neq[B] *[A]
$$

noncommutivity of rotations, finite rotations cannot be represented by vectors that follow the rules of vector addition.

Infinitesimal rotations can, however, be treated as vectors. The error in ignoring the order of small finite rotations is proportional to the angle of rotation squared. Angular velocities can be treated as vectors with no error. 


\section{EULER ANGLE CHOICE}

This instrument development uses a 3,2,1 Euler angle system to calculate buoy rotations (Goldstien, 1981). In a 3,2,1 Euler angle system, the object or reference frame is rotated firstly about its $\mathrm{z}$ axis by an angle $\phi$ (the three index), secondly about its new $\mathrm{y}$ axis by an angle $\theta$ (the two index), and thirdly about its new $x$ axis by an angle $\psi$ (the one index), Fig. 2-13. These sequential rotations can be combined to form a single
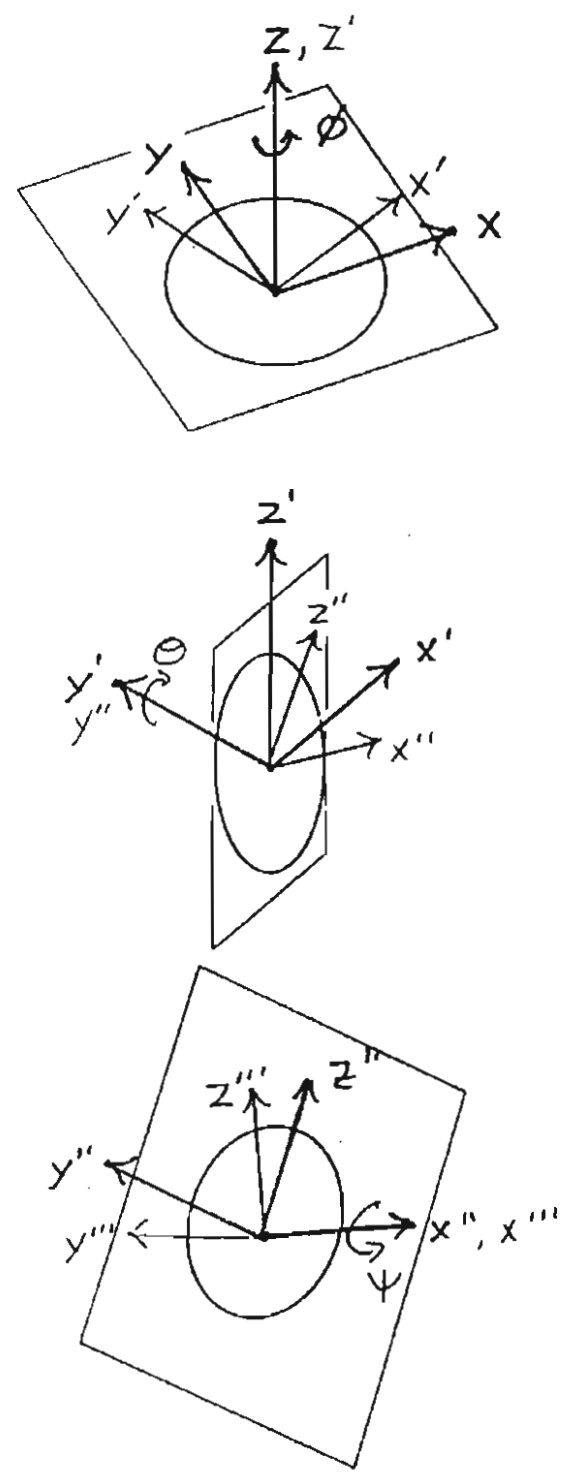

Fig. 2-13. Order of rotations for a 3,2,1 Euler angle system. 
rotational-transformation matrix (2-9). The body-centered rotation rates can be computed

$$
\begin{aligned}
& D=\left[\begin{array}{ccc}
\cos \phi & \sin \phi & 0 \\
-\sin \phi & \cos \phi & 0 \\
0 & 0 & 1
\end{array}\right] \\
& C=\left[\begin{array}{ccc}
\cos \theta & 0 & -\sin \theta \\
0 & 1 & 0 \\
\sin \theta & 0 & \cos \theta
\end{array}\right] \\
& B=\left[\begin{array}{ccc}
1 & 0 & 0 \\
0 & \cos \psi & \sin \psi \\
0 & -\sin \psi & \cos \psi
\end{array}\right] \\
& A=B C D=\left[\begin{array}{ccc}
\cos \theta \cos \phi & \cos \theta \sin \phi & -\sin \theta \\
\sin \psi \sin \theta \cos \phi-\cos \psi \sin \phi & \sin \psi \sin \theta \sin \phi+\cos \psi \cos \phi & \cos \theta \sin \psi \\
\cos \psi \sin \theta \cos \phi+\sin \psi \sin \phi & \cos \psi \sin \theta \sin \phi-\sin \psi \cos \phi & \cos \theta \cos \psi
\end{array}\right]
\end{aligned}
$$

from the Euler angles and Euler angle rates by (2-10). This matrix can be inverted to give

$$
\vec{\omega}=\left\{\begin{array}{c}
\omega_{x} \\
\omega_{y} \\
\omega_{z}
\end{array}\right\}=F(\psi, \theta, \phi)\left\{\begin{array}{c}
\dot{\psi} \\
\dot{\theta} \\
\dot{\phi}
\end{array}\right\}=\left[\begin{array}{ccc}
1 & 0 & -\sin \theta \\
0 & \cos \psi & \cos \theta \sin \psi \\
0 & -\sin \psi & \cos \theta \cos \psi
\end{array}\right]\left\{\begin{array}{c}
\dot{\psi} \\
\dot{\theta} \\
\dot{\phi}
\end{array}\right\}
$$

the Euler angle rates in terms of the Euler angles and the body-referenced angle rates (211). This new matrix will be called the Euler-angle update matrix in this thesis . Knowing

$$
\left\{\begin{array}{c}
\dot{\psi} \\
\dot{\theta} \\
\dot{\phi}
\end{array}\right\}=F^{-1}\left\{\begin{array}{c}
\omega_{x} \\
\omega_{y} \\
\omega_{z}
\end{array}\right\}=\left[\begin{array}{ccc}
1 & \tan \theta \sin \psi & \tan \theta \cos \psi \\
0 & \cos \psi & -\sin \psi \\
0 & \frac{\sin \psi}{\cos \theta} & \frac{\cos \psi}{\cos \theta}
\end{array}\right]\left\{\begin{array}{c}
\omega_{x} \\
\omega_{y} \\
\omega_{z}
\end{array}\right\}
$$

the initial attitude, successive attitudes can be stepped through with measured body- 
centered rotation rates.

The Euler-angle update matrix has some special properties. It should be noted that the $\mathrm{F}$ and $\mathrm{F}^{-1}$ matrices are not rotational-transformation matrices; their determinants are not equal to one and their inverse is not their transpose. There is a singularity in the Euler-angle update matrix when $\theta$ equals plus or minus $\pi / 2$, when the buoy is on its side. There is no heading $\phi$ in the Euler-angle update matrix. In normal operation, the heading angle can be much larger than either the pitch or the roll angle, and the calculation of heading is not as well conditioned (as will be shown in the magnetic compass section) as the calculation of pitch or roll. This 3,2,1 Euler angle system was chosen to minimize error propagation and to have its singularity at an attitude that the buoy has not been seen to approach in service. Also, if the Euler-angle update matrix is linearized, the error in calculating Euler angle rates is smaller than with other Euler angle systems.

Real buoy motions are smooth and only approximated by the discrete calculation steps in this algorithm. Commutivity error results from the difference between the smooth, real motion of the buoy and the discrete steps assumed by any Euler angle system. As mentioned above in this section, the difference between the 3,2,1 angle system and a $1,2,3$ angle system is proportional to the rotation angle squared. Average body-centered rotation rate is measured at each time step and used in the algorithm. The error in each step of the algorithm should also be proportional to the angle squared in that time step. Increasing the calculation step rate increases the number of errors with the number of steps, and decreases the error of each step by one over the number squared. The result of both is a total commutivity error for a buoy evolution, that is proportional to the angular displacement of each time step.

\section{OTHER TECHNIQUES TO CALCULATE ATTITUDE}

There exist several other choices of algorithms to calculate the attitude of a strapdown inertial system, including: direct direction-cosine-matrix updates, fourparameter techniques, and other Euler angle systems (McKern, 1968). The direct 
direction-cosine-matrix update and the four-parameter systems such as the Euler parameter technique have the advantages of no singularities and fewer calculations with transcendental functions. Both techniques are popular in real-time inertial-navigation systems that require an unrestricted operational envelope, and that may have limited processing power. As mentioned earlier, the singularity of the chosen 3,2,1 Euler angle system is an attitude that the buoy has not approached in the ocean, therefore the existence of this singularity is not a problem.

The signal processing for this instrument was performed after the buoy was recovered, on an 80486 computer in the Matlab environment. Before deciding which inertial attitude computation technique to use, a test was performed to measure how much transcendental functions would slow the computation. A series of tangents and adds of vectors of random numbers took less than twice as long as the same number of multiplies and adds of the same vectors. I do not know if this is more a function of Matlab's overhead or efficient processing of transcendental functions by the floating point processor. There is no significant increase in processing speed when using these less intuitive algorithms in this processing environment. Additionally, Euler angles use less memory. On a more general inertial-measurement system, these other techniques would have to be considered.

Two other Euler-angle systems that I considered but rejected were the 3,1,3 system and the $1,2,3$ system (Pio, 1966). The 3,1,3 system is used in many dynamics textbooks and is convenient for describing the motion of a spinning top. The order of rotations is about the $z$ axis, the new $x$ axis, and then the new $z$ axis. The 3,1,3 Eulerangle update matrix however, has a singularity at the operating point of floating right side up, and was therefore dismissed. The 1,2,3 Euler-angle system rotates about the $\mathrm{x}$ axis, then about the new $y$ axis, and then about the new $z$ axis. This system, however, has the heading angle $\phi$ in the Euler-angle update matrix. The heading ranges from 0 to 360 degrees while pitch and roll seldom exceed 20 degrees. Additionally, as the next section explains, the measurement of heading is poorly conditioned as compared to the 
measurement of pitch and roll, resulting in greater heading error. The 3,2,1 Euler-angle system results in smaller errors in buoy attitude processing than either the 3,1,3 or the $1,2,3$ systems, and was therefore chosen.

\section{MAGNETIC COMPASSES}

Magnetic compasses work by projecting the magnetic lines of flux on a plane that is perpendicular to the perceived gravitational field. In Woods Hole MA., the magnetic lines of flux are inclined from the horizontal by about seventy degrees, making this measurement poorly conditioned. This is why aircraft need gyrocompasses to measure their heading in a turn. The vorticity meter has a three axis fluxgate magnetometer. The inertial processing of the instrument, described in the Inertial Algorithm section, rotates the measured magnetic fluxes into a horizontal plane and then computes heading. An error in pitch or roll in the algorithm used is equivalent to having the magnetometer at the attitude of the pitch or roll error, which can result in a heading error that is greater than the pitch or roll error.

This section calculates an example of what happens if instrument heading is calculated from magnetometer measurements made in a plane that is tilted and the tilt is not accounted for. The following derivation uses the coordinate system shown in Fig 214. With this coordinate system, the heading of a horizontal plane can be computed

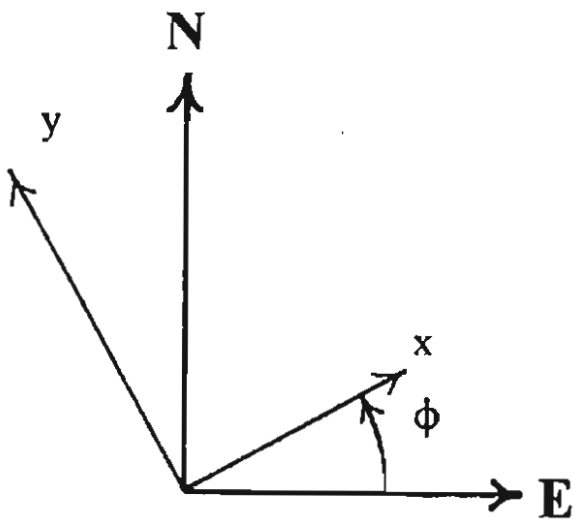

Fig. 2-14. Coordinate system used in compass example 
from the inverse tangent of the $\mathrm{x}$-direction flux divided by the $\mathrm{y}$-direction flux. If the plane of the $\mathrm{x}$ and $\mathrm{y}$ magnetometer is put at an angle to horizontal, the measured fluxes will be given by equation (2-12). In this equation, $B_{x}, B_{y}$, and $B_{z}$ are the magnetic fluxes in the

$$
\begin{aligned}
& B_{x} \\
& B_{y}=[A]
\end{aligned}\left\{\begin{array}{c}
0 \\
B_{z}
\end{array}\left\{\begin{array}{c}
0 \\
540 m G \cos \left(-70^{\circ}\right) \\
\sin \left(-70^{\circ}\right)
\end{array}\right\}\right.
$$

rotated (buoy attitude direction) $\mathrm{x}, \mathrm{y}, \mathrm{z}$, and $[\mathrm{A}]$ is the rotational-transformation matrix described earlier. The measured heading will then be given by equation (2-13). In this

$$
\begin{aligned}
& \phi_{m}=\tan ^{-1} \frac{B_{x}}{B_{y}} \\
& \Phi_{m}=\tan ^{-1}\left(\frac{\cos \theta \sin \phi_{t} \cos \left(-70^{\circ}\right)-\sin \theta \sin \left(-70^{\circ}\right)}{\left(\sin \psi \sin \theta \sin \phi_{t}+\cos \psi \cos \phi\right) \cos \left(-70^{\circ}\right)+\cos \theta \sin \psi \sin \left(-70^{\circ}\right)}\right)
\end{aligned}
$$

equation, $\phi_{1}$ is the true heading, $\phi_{\mathrm{m}}$ is the measured heading. The instantaneous heading error, at some headings, for one degree of pitch or roll is $2.75^{\circ}$. An accuracy specification of three degrees in heading will require pitch and roll accuracy of one degree.

Because of the nonlinearities in equation (2-13), taking the mean of measured heading of a rocking buoy, and not compensating for pitch and roll, can result in significant error. Fig. 2-15 shows the results of a fifteen degree buoy oscillation in $\theta$ when the true heading $\left(\phi_{t}\right)$ is 55 degrees. The rocking angle $\theta$, the true heading, and the measured heading are shown through the rocking cycle. The error in mean-measured heading is over eight degrees. Not knowing which way is up can result in significant error in measured heading, even in the mean. 


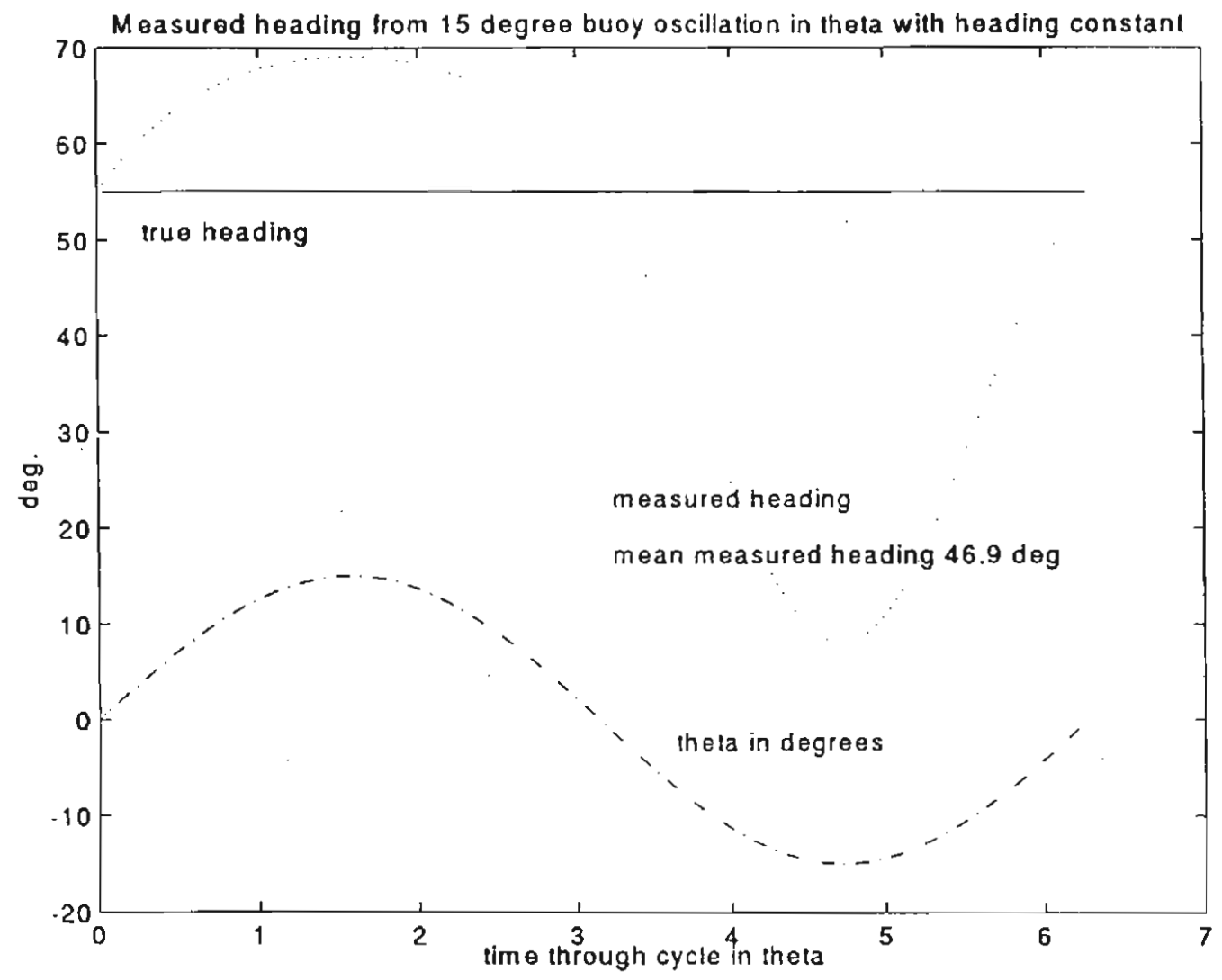

Fig. 2-15. Measured heading for rocking buoy if heading is calculated from $\tan ^{-1} \mathrm{~B}_{\mathrm{x}} / \mathrm{B}_{\mathrm{y}}$ and pitch and roll are not accounted for.

\section{CONING MOTIONS}

One motive for measuring and compensating for instrument attitude is to avoid the measurement errors that can result from coning motions (Goodman and Robinson, 1958). Coning motion occurs when a line in the object moves in space as to follow the surface of a cone. This section describes one such coning motion and calculates the error that results if the motion is not accounted for. The Euler angles in this example have a time history described by equation (2-14), where $\psi$ is a sine curve and $\theta$ is a cosine curve. The measured body-referenced angle rates are given by equation (2-15). Plugging in the 


$$
\begin{array}{ll}
\psi=a \sin \left(\omega_{o} t\right) & \dot{\psi}=a \omega_{a} \cos \left(\omega_{o} t\right) \\
\theta=a \cos \left(\omega_{o} t\right) & \dot{\theta}=-a \omega_{o} \sin \left(\omega_{o} t\right) \\
\phi=0 & \dot{\phi}=0
\end{array}
$$

$$
\left\{\begin{array}{c}
\omega_{x} \\
\omega_{y} \\
\omega_{z}
\end{array}\right\}=[F]\left\{\begin{array}{c}
\dot{\psi} \\
\dot{\theta} \\
\dot{\phi}
\end{array}\right\} \begin{aligned}
& \dot{\psi}-\dot{\phi} \sin \theta \\
& \dot{\theta} \cos \psi+\dot{\phi} \cos \theta \sin \psi \\
& -\dot{\theta} \sin \psi+\dot{\phi} \cos \theta \cos \psi
\end{aligned}
$$

values given, assuming small coning motion angles, and taking the small angle approximation of $\sin (x)=x$ and $\cos (x)=1$, results in equation $(2-16)$. The body-referenced

$$
\begin{aligned}
& \omega_{x}=a \omega_{0} \cos \left(\omega_{o} t\right) \\
& \omega_{y}=-a \omega_{o} \sin \left(\omega_{0} t\right) \\
& \omega_{z}=a^{2} \omega_{0} \sin ^{2}\left(\omega_{0} t\right)
\end{aligned}
$$

$\mathrm{z}$ axis measures a time-averaged rotation rate while the true heading $\phi$ is zero and constant. If the inertial measurement system does not keep up with buoy motion, this error can result. If a buoy measures vorticity, ignores instantaneous attitudes and angle rates, and only compensates measured relative vorticity for average drift in heading, it would calculate a earth-referenced vorticity of minus two times this angle rate $\left(\omega_{z}\right)$ in a still fluid.

\section{ALGORITHM ERROR PROPAGATION}

Because the Euler angle rates used to calculate future Euler angles depend on the current Euler angles, an error in the current estimate of Euler angles can propagate into the future. This section describes a perturbation expansion of the Euler angle update algorithm to estimate how errors propagate. Equation $(2-17)$ is the continuous limit of the

$$
\dot{\Psi}=\left[F^{-1}(\Psi)\right]\{\omega\}=[G(\Psi)]\{\omega\}
$$


Euler-angle update algorithm. The Euler angle is broken into the true Euler angle $\Psi$ and a small error $\delta$ equation (2-18) and plugged into equation (2-17) to give equation

$$
\begin{gathered}
\Psi=\Psi+\delta=\left\{\begin{array}{l}
\Psi \\
\theta \\
\phi
\end{array}\right\}+\left\{\begin{array}{l}
b \\
c\} \\
d
\end{array}\right\} \\
\dot{\Psi}=\dot{\Psi}+\dot{\delta}
\end{gathered}
$$

(2-19). This equation is solved for how the error changes using a Taylor expansion for $G$

$$
\dot{\Psi}+\dot{\delta}=[G(\Psi+\delta)]\{\omega\}
$$

and dropping second and higher order terms in error (2-20). From equation (2-20) we can

$$
\begin{gathered}
\dot{\delta}=\left[\frac{\partial G(\Psi)}{\partial \Psi} \delta\right] \omega \\
\dot{b}=\left[\begin{array}{ccc}
0 & b \tan \theta \cos \psi+c \sec ^{2} \theta \sin \psi & -b \tan \theta \sin \psi+c \sec ^{2} \theta \cos \psi \\
0 & -b \sin \psi & -b \cos \psi \\
0 & b \frac{\cos \psi}{\cos \theta}+c \frac{\sin \psi}{\cos \theta} \tan \theta & -b \frac{\sin \psi}{\cos \theta}+c \frac{\cos \psi}{\cos \theta} \tan \theta
\end{array}\right]\left\{\begin{array}{c}
\omega_{x} \\
\omega_{y} \\
\omega_{z}
\end{array}\right\}
\end{gathered}
$$

estimate how a small error propagates during a specified body motion. We can also conclude that errors in the heading Euler angle $d$, do not propagate to roll or pitch estimates because the heading $\phi$ is not in the Euler-angle update matrix.

The first example coning motion to be discussed will be the coning motion example from Coning Motions section. The angles and angle rates are reviewed in equation (2-21). In this equation, $a$ is the angular amplitude and $\omega$ is the frequency of the

$$
\begin{array}{lll}
\psi=a \sin (\omega t) & \psi=a \omega \cos (\omega t) & \omega_{x}=a \omega \cos (\omega t) \\
\theta=a \cos (\omega t) & \dot{\theta}=-a \omega \sin (\omega t) & \omega_{y}=-a \omega \sin (\omega t)+o\left(a^{3}\right) \\
\phi=0 & \dot{\phi}=0 & \omega_{z}=a^{2} \omega \sin ^{2}(\omega t)
\end{array}
$$


coning motion. The last relations are good to order $\alpha^{2}$ in that a small angle approximation was made. Using these parameters in equation 2-20, making the small angle approximation, and dropping terms that have a time average of zero results in equation (222) showing the Euler angle errors stay constant. Dropping the zero time-average terms

$$
\begin{aligned}
& \dot{b}=0 \\
& \dot{c}=0 \\
& \dot{d}=0
\end{aligned} \quad+o\left(a^{3}\right)
$$

is reasonable because under these assumed motions, the errors $b, c$, and $d$ will change much slower than $\omega$ by at least order $a^{3}$.

The second example of coning motion is one with zero time-average, buoyreferenced, $z$-axis rotation rate. The angles for this motion are shown in equation (2-23).

$$
\begin{array}{llll}
\Psi=a \sin (\omega t) & \dot{\psi}=a \omega \cos (\omega t) & \omega_{x}=a \omega \cos (\omega t) & +o\left(a^{3}\right) \\
\theta=a \cos (\omega t) & \dot{\theta}=-a \omega \sin (\omega t) & \omega_{y}=-a \omega \sin (\omega t) & +o\left(a^{3}\right) \\
\phi=\int a^{2} \omega \sin ^{2}(\omega t) d t & \dot{\phi}=a^{2} \omega \sin ^{2}(\omega t) & \omega_{z}=0 & +o\left(a^{3}\right)
\end{array}
$$

Plugging this motion into equation (2-20), making the small angle approximation, and dropping terms of order $a^{3}$ and smaller gives the angular error propagation (2-24). These

$$
\begin{aligned}
\dot{b} & =-c a^{2} \omega \sin ^{2}(\omega t) \\
\dot{c} & =b a^{2} \omega \sin ^{2}(\omega t) \\
\dot{d} & =0
\end{aligned}
$$

equations were calculated for an initial error of 0.05 radians in $\Psi$ and the results are shown in Fig. 2-16. This solution shows that under certain buoy motions, slow oscillating errors in Euler angles can occur. 


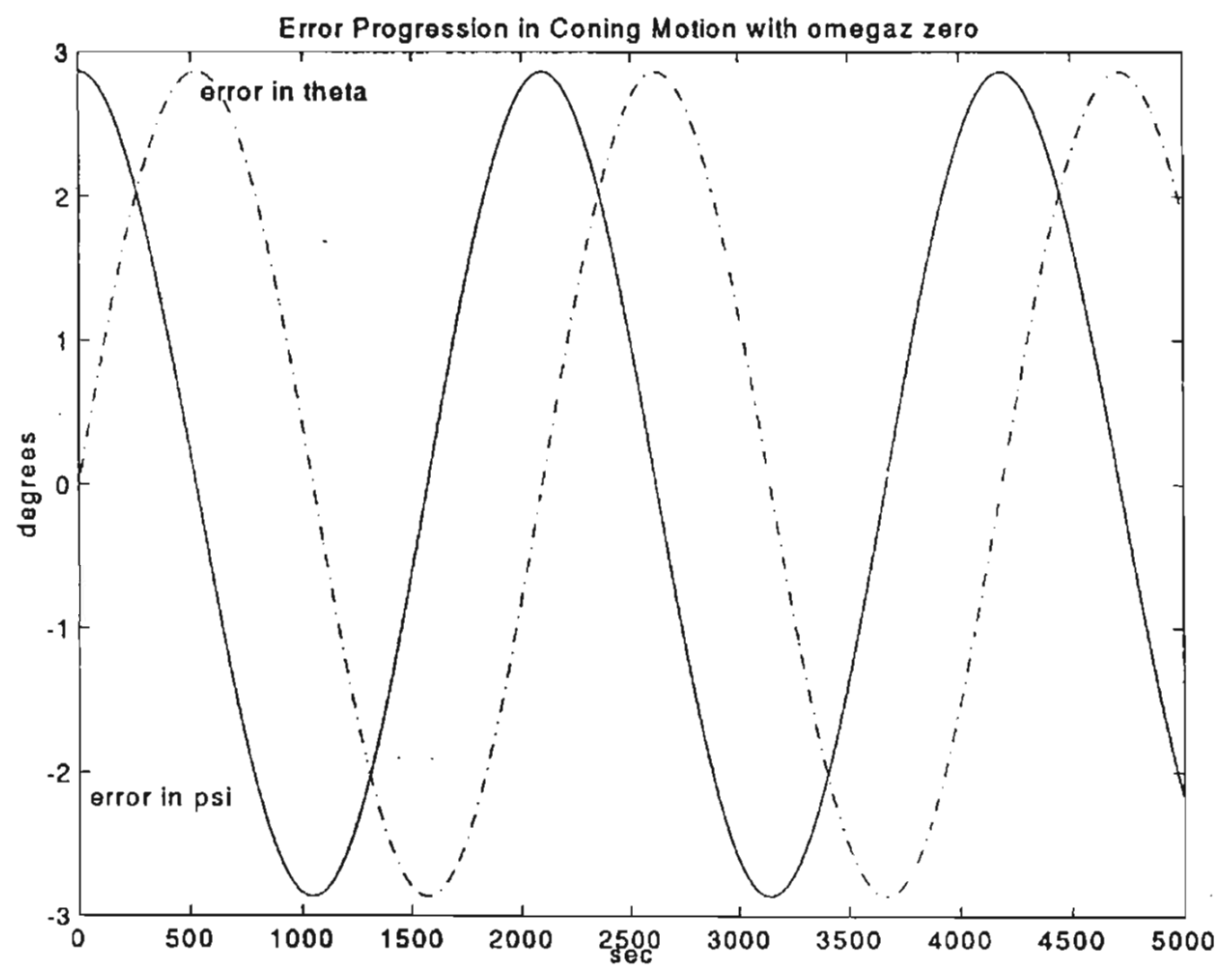

Fig. 2-16. Error progression in coning motion with zero time-average $z$-axis rotation rate. 


\section{INERTIAL ALGORITHM}

This section describes the algorithm used to compute the buoy attitude and motion

that are in turn used to remove buoy motion from measured fluid motion. Complementary filtering allows the use of noisy sensors with drift and still meet the accuracy specifications mentioned in the Inertial Measurement Unit section. Using more accurate sensors would increase cost and require more power. The rate gyros have a manufacturer drift specification of 0.1 degrees per second. To meet the three-degree accuracy specification, a conventional strapdown algorithm that just integrates rate gyro output, would only meet the accuracy specification for thirty seconds. The use of these noisy, drifty rate gyros requires information from other sensors to compensate for gyro drift. The algorithm I use to compute buoy attitude and motion makes assumptions about the buoy motion that allow the use of accelerometers and magnetometers to compensate for gyro drift. For a general use inertial system, such as one for an aircraft, these motion constraints could not be made and this algorithm could not be used.

\section{CONSTRAINTS OF BUOY MOTION}

Constraints on buoy motion allow the inertial system to align itself in a seaway. These motion assumptions are based on the measured information that there is almost no wave energy below a twenty-five second period. This analysis ignores tides because tides would not tilt the buoy and the inertial measurement unit is not accurate enough to measure accelerations at a frequency as low as a tidal frequency. The motion assumptions for periods longer than twenty-five seconds are zero average heave (vertical displacement), zero average heave velocity, and zero average horizontal velocity (surge and sway). Currents on the order of several tenths of a meter per second that vary over periods of hours or days cannot be measured by this inertial measurement unit, and are assumed to be zero.

\section{COMPLEMENTARY FILTERS}

The algorithm uses complementary filters to mix redundant estimates of Euler angles from rate gyros, accelerometers, and magnetometers. A block diagram of a 
complementary filter is shown in Fig. 2-17. In this figure, $s(t)$ is the true signal, $n_{1}$ or $2(t)$

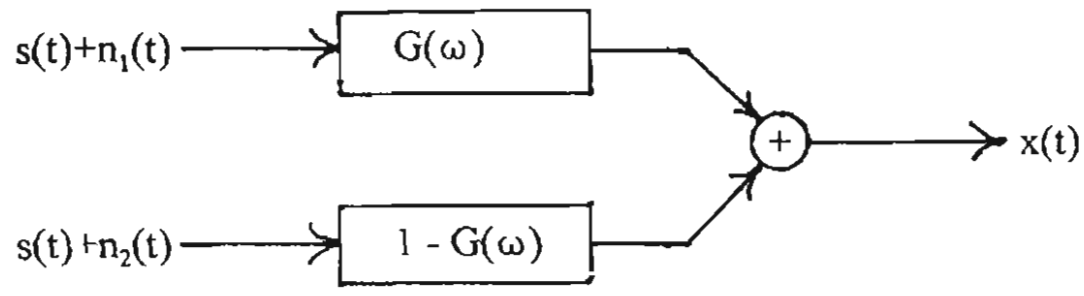

Fig. 2-17. Complementary filter of redundant sensor measurements

are noise added to the signal, $G(\omega)$ is the transfer function of filter one, and $x(t)$ is the output. Weiner filter theory gives the result equation (2-25) for an optimal non-causal

$$
G(j \omega)=\frac{S_{n_{2} n_{2}}}{S_{n_{1} n_{1}}+S_{n_{2} n_{2}}}
$$

filter (Brown, 1983). In this equation, $S_{n_{i} n_{i}}$ is the power spectral density of noise in signal I. This solution assumes that noise from the two sensors $n_{1}$ and $n_{2}$ are uncorrelated. Noncausal filters were chosen over causal filters because the processing does not need to be done in real time, to avoid phase shifts, and to reduce mean square error. Mean square error from an optimal non-causal filter is a little smaller than from an optimal causal filter. A Kalman filter was not needed because one can say a priori in what frequency bands each sensor has less noise.

A conceptual block diagram of the inertial processing is shown in Fig. 2-18. The high pass and low pass filters are complementary and I justify their form in the next section. The rate gyros are integrated to make angle estimates of pitch, roll, and heading. 
Lowpass

filter

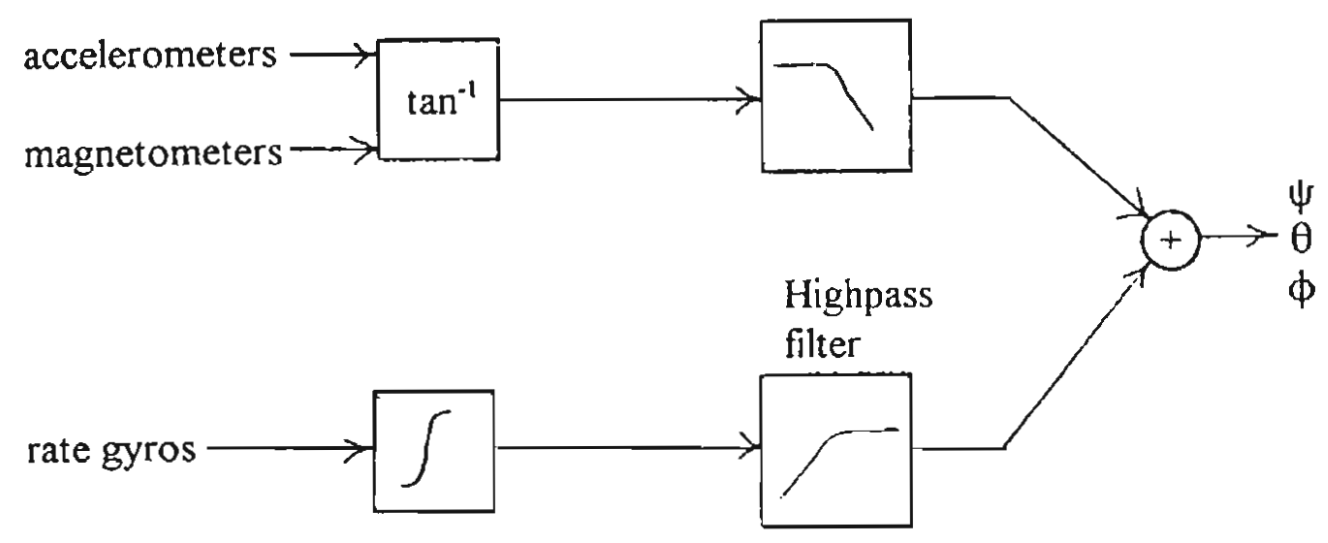

Fig. 2-18. Conceptual block diagram of inertial processing

A second estimate of pitch and roll is made by taking the inverse tangent of the horizontal acceleration divided by the vertical acceleration. This estimate has errors when the buoy experiences wave acceleration. A second heading estimate is made by taking the inverse tangent of measurements of orthogonal, horizontal, magnetic-flux measurements. Heading estimates must have their branch cuts unwrapped before filtering. The next section models the noise of the angle estimates, in a seaway.

\section{MODEL OF WAVE MOTION CAUSED ANGLE ERROR}

This section models noise in the computation of buoy angle to justify the complementary filter form used in the inertial processing. The model is of a buoy in a two-dimensional wave field, assumes that the buoy horizontal acceleration follows the water wave horizontal acceleration, assumes that the buoy angle is computed from redundant measurements of a rate gyro and horizontal and vertical accelerometers, and linearizes some of the computations. The rate gyro has close to white noise, and its noise is modeled as white with the same variance as the measured sensor noise. After integrating the rate gyro output to estimate angle, the 
noise spectrum is given by equation (2-26). In this equation, $N_{r r}$ is the noise power

$$
\psi_{r}=\frac{N_{r}(j \omega)}{(j \omega)^{2}}=\frac{9.5 * 10^{-8} \mathrm{rad}^{2} / \mathrm{sec}}{\omega^{2}}
$$

spectral density of the rate gyro and $\Psi_{r r}$ is the noise spectrum of the angle estimate from the integrated rate gyro. In this model every angle and frequency is expressed in radians for convenience. The accelerometer-derived angle is the inverse tangent of buoy-axis horizontal acceleration divided by buoy-axis vertical acceleration (2-27). This equation

$$
\psi=\tan ^{-1} \frac{\ddot{x}}{\ddot{z}} \approx \frac{\ddot{x}}{g}
$$

will be linearized for the model leaving the horizontal acceleration divided by the acceleration due to gravity. The model's assumed wave spectrum is a Pierson-Moskowitz spectrum whose energy is given by equation (2-28) (Newman, 1977). In this equation, $\alpha$

$$
S(\omega)=\frac{\alpha g^{2}}{\omega^{5}} e^{-\beta\left(\frac{g}{U \omega}\right)^{4}}
$$

and $\beta$ are empirical constants assumed to be $8.1 \times 10^{-3}$ and 0.74 respectively, $g$ is the acceleration due to gravity, $U$ is the windspeed at 19.5 meters, and $\omega$ is the radian frequency. The angle noise spectral density $\Psi_{n n}$ from this lateral wave acceleration is then given by equation (2-29). Accelerometer electronic noise is modelled as white with the

$$
\psi_{n n}=\frac{2 \alpha}{\omega} e^{-\beta\left(\frac{g}{U \omega}\right)^{4}}
$$

measured variance having a noise spectral density of $4.2 \times 10^{-8} \mathrm{rad}^{2} \mathrm{~s}$. These noise spectra are plotted as a function of the radian frequency for two windspeeds in Fig. 2-19. For the 


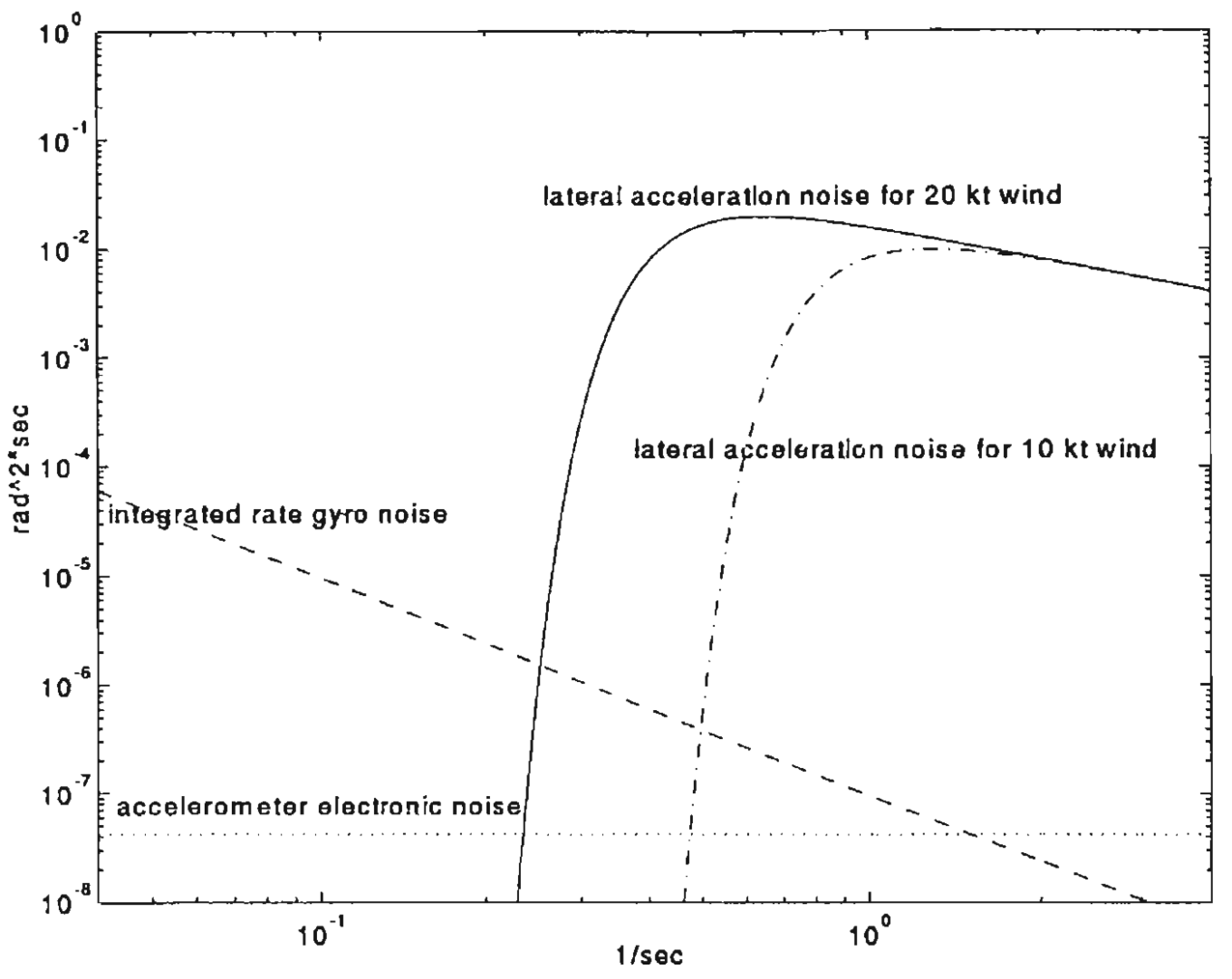

Fig. 2-19. Angle noise for buoy in a two-dimensional seaway subjected to the horizontal accelerations of a Pierson-Moskowitz spectrum.

higher windspeed, the lateral acceleration spectrum moves up and to the left. This model of measured angle noise justifies lowpass filtering the angle estimate from the accelerometers and highpass filtering the angle estimate from the integrated rate gyro. Returning to describing the inertial processing algorithm, the actual inertial processing uses a fourth-order, butterworth, highpass filter with a thirty-second period on the integrated rate-gyro output, and its complement on the other estimates of Euler angles.

\section{HORIZONTAL REFERENCE FRAME FOR HEADING}

As described in the Magnetic Compasses section, to get an accurate measurement of heading from magnetic flux requires that the magnetic flux measurements be in a horizontal plane. This is done by first calculating pitch and roll angles, and then using 
these angles to rotate the buoy-referenced magnetometer measurements into an intermediate, horizontal, computational reference frame (2-30). In this equation, xyz refers to the buoy axis and 'x'y'z' refers to the intermediate computational reference frame.

$$
\begin{gathered}
B_{i n t}=A_{21} * B_{x y z} \\
\left\{\begin{array}{c}
B_{x^{\prime}} \\
B_{y^{\prime}} \\
B_{z^{\prime}}
\end{array}\right\}=\left[\begin{array}{ccc}
\cos \theta & \sin \psi \sin \theta & \cos \psi \sin \theta \\
0 & \cos \psi & -\sin \psi \\
-\sin \theta & \sin \psi \cos \theta & \cos \psi \cos \theta
\end{array}\right]\left\{\begin{array}{l}
B_{x} \\
B_{y} \\
B_{z}
\end{array}\right\} \\
\phi_{s}=\tan ^{-1} \frac{B_{x^{\prime}}}{B_{y^{\prime}}}
\end{gathered}
$$

\section{INERTIAL ALGORITHM}

The complete inertial algorithm is shown as a block diagram in Fig. 2-20. The simpler, conceptual block diagram of Fig. 2-18 linearizes the Euler-angle-update matrix as the identity matrix, but gives a reasonable first estimate of the angles. The first angle estimate is then used in the nonlinear, Euler-angle-update matrix and the calculation is repeated. In simulation, the algorithm is stable and is more accurate than linearized versions. In the figure, the $A_{\text {int }}$ rotates the buoy-based magnetometer measurements into a horizontal, computational frame. The slow $\theta_{\mathrm{s}}$ measurement uses the inverse tangent of the $\mathrm{x}$ acceleration multiplied by $\cos \psi$ divided by the vertical acceleration. This is exact and results from a nonlinearity in the 3,2,1 Euler angle system. The first and last minute of each processed record is ignored because of filter transients. This algorithm meets the accuracy needed while using low-power, noisy, and drifty sensors. 


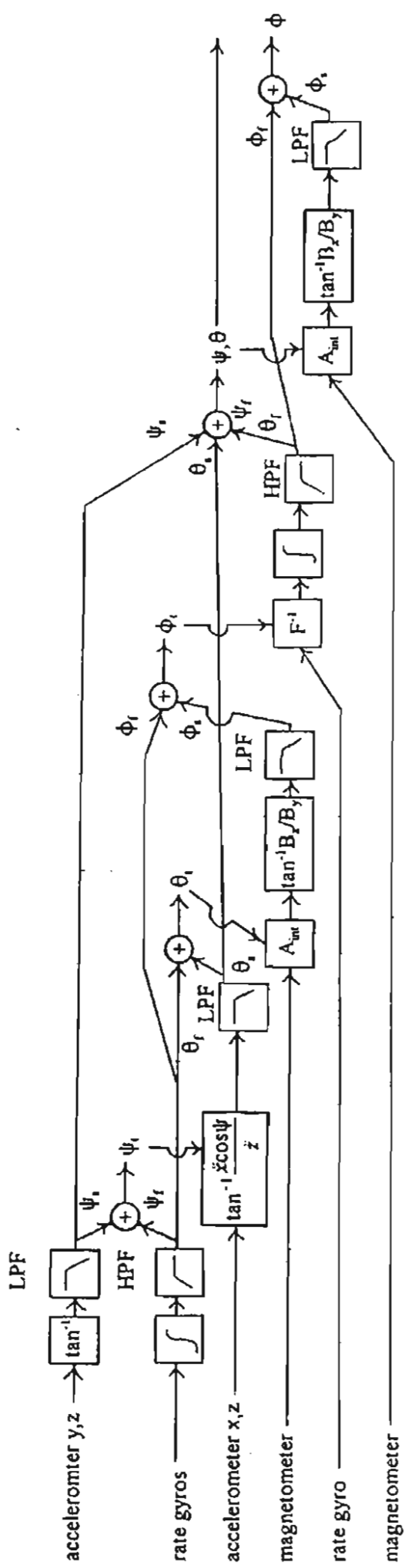

Fig. 2-20. Block diagram of Euler-angle inertial algorithm. 


\section{DATA PROCESSING}

The data processing for the instrument is represented in a block diagram in Fig. 221. The raw data is transferred from the data logger hard disk to a personal computer hard disk and then converted into Matlab readable files with the programs offld6.exe and

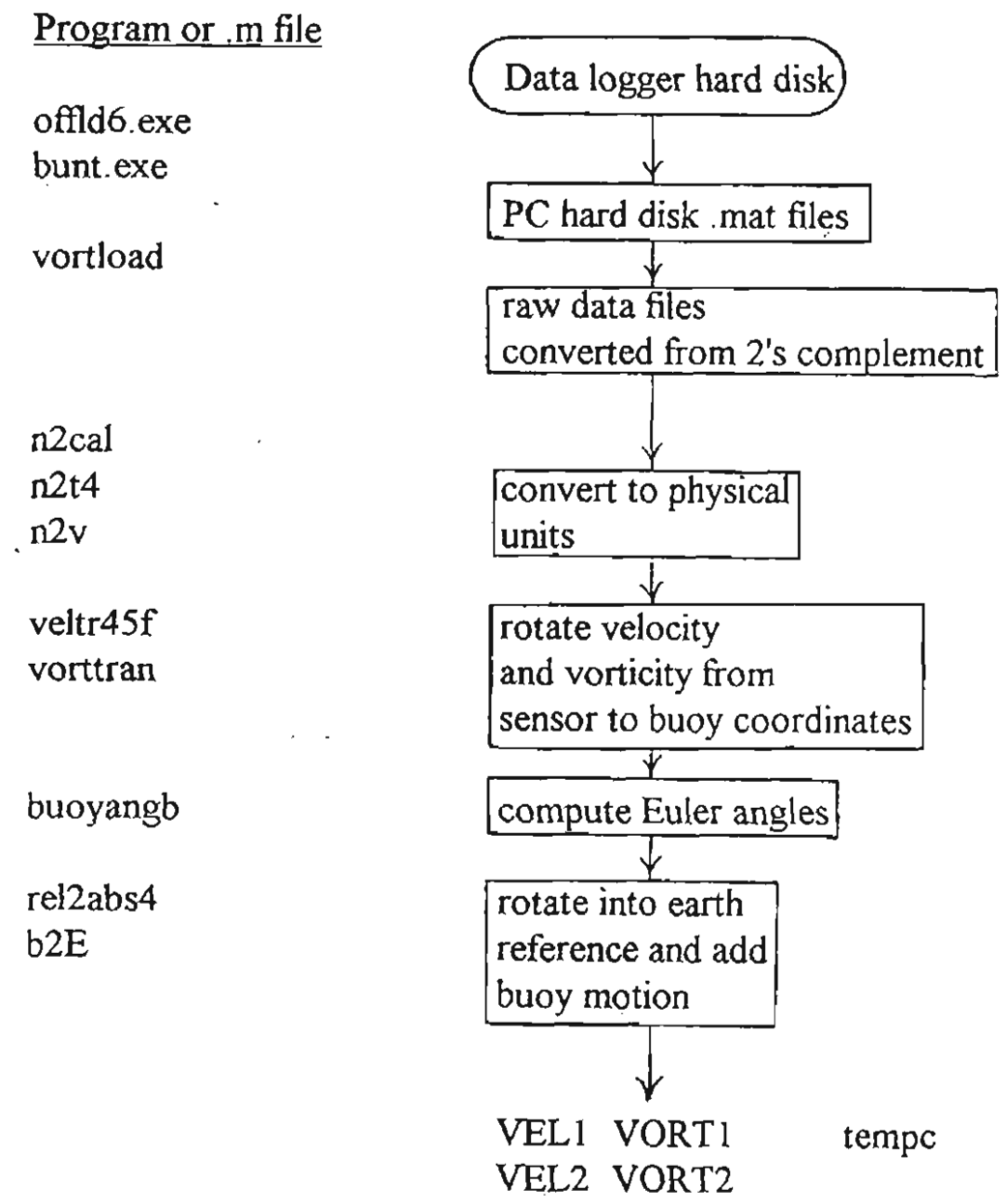

Fig. 2-21. Vorticity meter data path

bunt.exe. Data is then loaded into the Matlab environment and the two's complement numbers converted to integers with the file vortload.m. In the Matlab environment, $x x x . m$ files are sequences of processing commands that Matlab interprets and executes. The processing files are listed in appendix B along with descriptions of all intermediate 
variables. These integers are then converted into physical units with the files $\mathrm{n} 2 \mathrm{cal} . \mathrm{m}$, $\mathrm{n} 2 \mathrm{t} 4 . \mathrm{m}$, and $\mathrm{n} 2 \mathrm{v} . \mathrm{m}$. The buoy-relative fluid velocities and vorticities are rotated from sensor coordinates to buoy coordinates with the files veltr $45 \mathrm{f} . \mathrm{m}$ and vorttran.m. There are redundant velocities that are weighted by their direction cosines in computing the buoyreferenced, relative velocity. Euler angles are computed by the file buoyangb.m. These Euler angles are then used to rotate buoy relative velocity and vorticity into Earth coordinates, adding buoy velocity and rotation with the files rel2abs4.m and b2E.m. Twice the buoy rotation rate is added to vorticity to compensate for buoy motion, because the vorticity of a nondeforming, rotating block of fluid is twice the rotation rate. The final output consists of Earth-referenced velocities VEL1 and VEL2 in $\mathrm{cm} / \mathrm{s}$, Earth-referenced vorticities VORT 1 and VORT2 in $1 / \mathrm{s}$, and temperature at the top and bottom of each measurement volume tempc in degrees $C$. The numbers 1 and 2 in the velocity and vorticity variables refer to sensors 1 and 2; sensor 1 is adjacent to the instrument case and sensor 2 is adjacent to the float. The earth-based coordinate system is direction 1 east, direction 2 north, and direction 3 up. In general, variables in lowercase letters are in buoy or sensor coordinates and variables in capitals are in earth-based coordinates of east, north and vertical.

The last processing stage involves rotating buoy relative velocity and adding buoy motion, using the rel2abs4.m file. Velocity in an inertial reference frame can be computed from velocity in a moving and rotating reference frame by equation (2-31). The Earth-

$$
\vec{v}=\frac{d \vec{R}_{o}}{d t}+\vec{v}_{r c l}+\vec{\omega} \times \vec{r}
$$

reference velocity $\vec{v}$ is equal to the velocity of the moving frame $\frac{d \vec{R}}{d t}$, and the velocity relative to the moving frame $\vec{v}_{r e l}$, and the cross product of the rotation rate of the moving frame with the position vector to the point. The buoy velocity is defined at the accelerometer block, so $\vec{r}$ is the vector from the accelerometers to the center of the measurement volume. The processing of rel2abs $4 . \mathrm{m}$ is shown as a block diagram in Fig. 
2-22. In the top half of the figure, the $\omega \times r$ term is added to buoy-relative flow in the buoy frame and then rotated into Earth coordinates. In the bottom half of the figure, the

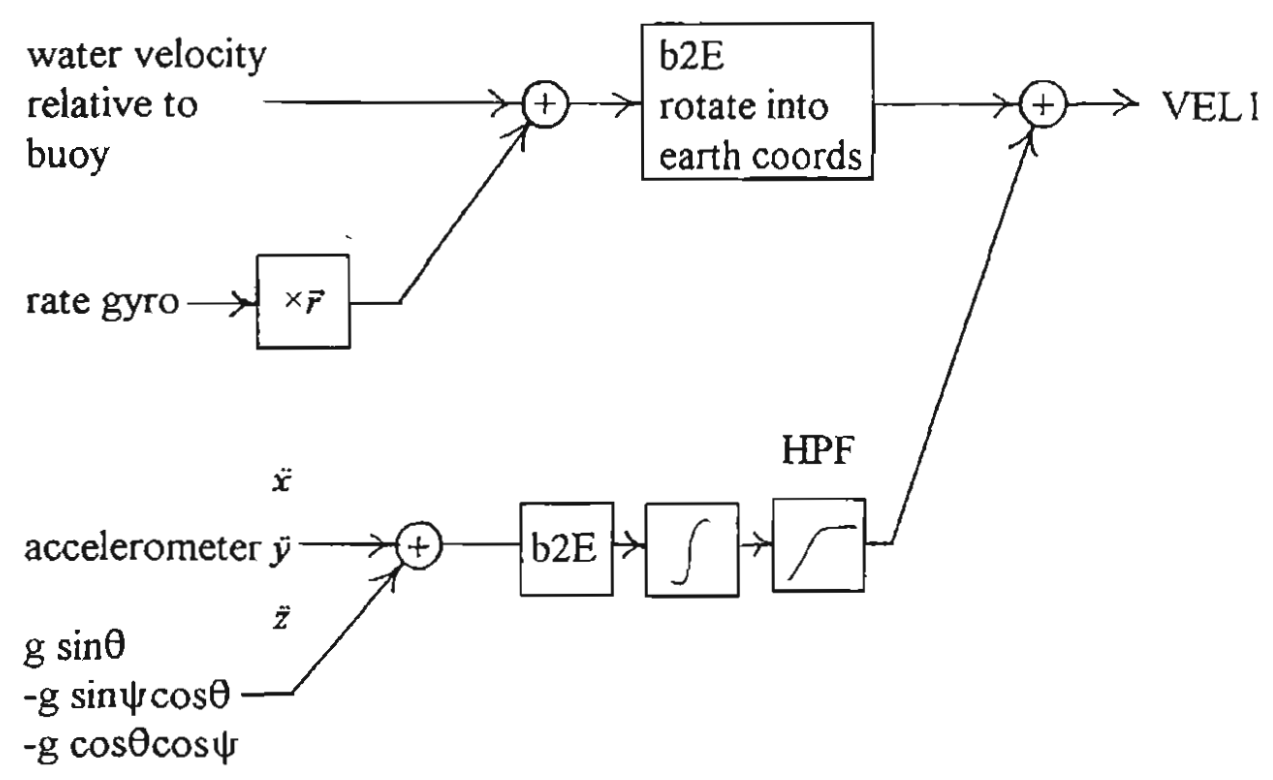

Fig. 2-22. Algorithm to compute Earth-referenced flow velocity

acceleration due to gravity in the buoy reference frame is subtracted from the buoy reference accelerations. The net buoy acceleration is then rotated into earth coordinates, integrated into buoy velocity, high-pass filtered in accordance to the zero-average velocity assumption. This final buoy velocity is added to relative buoy flow velocity to form the Earth-referenced absolute velocity. As mentioned earlier, this velocity does not include drift currents that have a period slower than thirty seconds; and, the Earth reference frame is considered inertial. 


\section{SIMULATION OF INERTIAL PROCESSING}

This section describes numerical simulations of the inertial processing. I conducted simulations to help develop the algorithm used and to estimate the accuracy of the algorithm in wave motion. In these simulations, I also evaluated errors due to possible coning motions, and the effectiveness in using an intermediate-computational, reference frame to calculate heading. The major causes of algorithm error are broken down into the following types of errors: commutivity, integration, round-off, and sensor lag. Lastly, simpler inertial algorithms are simulated to evaluate the tradeoff between algorithm complexity and accuracy.

Simulation of the inertial processing is shown as a block diagram in Fig. 2-23. The buoy is subjected to representative wave motions, sensed physical quantities are calculated, sensor noise added, and then these simulated measurements are processed by the normal buoy software. These simulated processed variables can then be compared to the input simulated buoy motion to measure the inertial system accuracy. In the

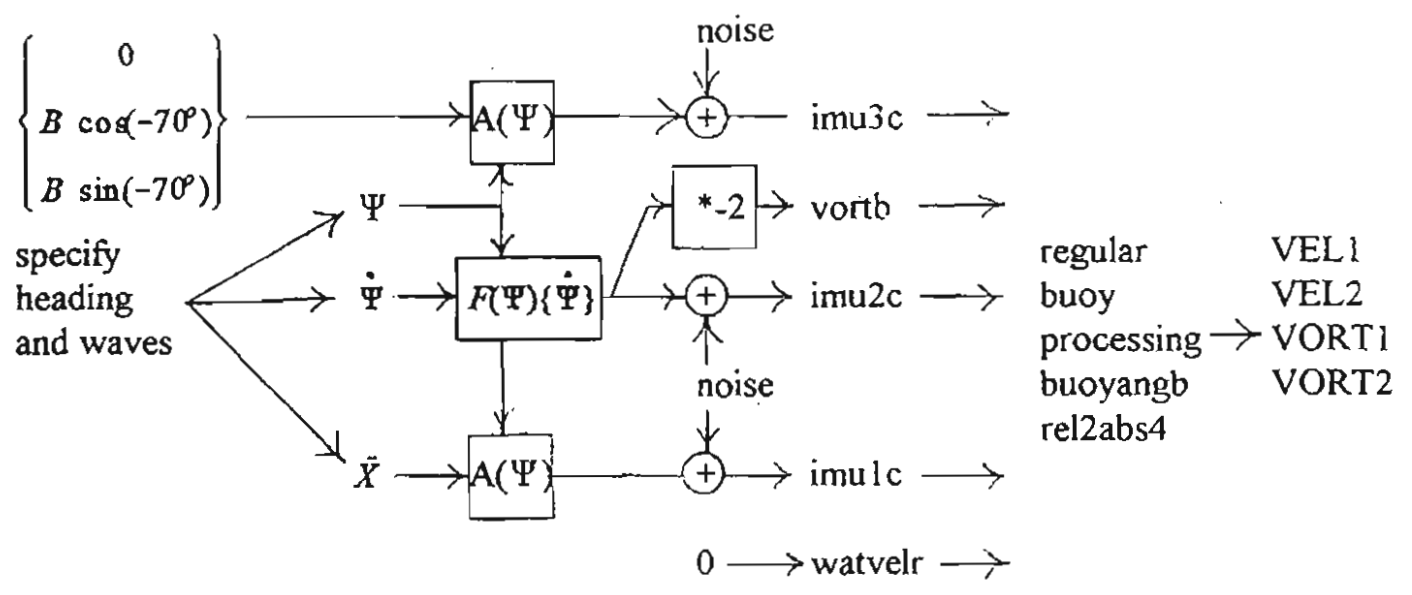

Fig. 2-23. Inertial processing simulation block diagram. Imulc, imu2c, and imu3c are accelerometer, rate gyro, and magnetometer, vortb and watvelr are buoyrelative vorticity and velocity, VEL1, VEL2, VORT1, and VORT2 are computed, earth-referenced velocity and vorticity. 
simulations, I used four deep-water waves, two in the $+X$ direction (east) and two in the $+Y$ direction (north). The simulated heading of the buoy is the measured buoy heading from a deployment South of Martha's Vineyard. The buoy is assumed to be a surface heave follower and that the horizontal velocity of the top sphere and bottom instrument case follow the horizontal water velocity at their average depths. Assuming that the buoy top and instrument case follow the wave horizontal excursions at their respective depths,

the contributions to buoy angles are given by $\frac{1}{L}\left(1-e^{k z}\right)$ multiplied by wave amplitude, for each wave. In this expression, $L$ is the distance from the buoy top to the instrument case and $k$ is the wavenumber. The simulated measured water velocity relative to the buoy is zero. This zero relative velocity exaggerates what the actual vertical velocity would be at depth, because it ignores the $\mathrm{e}^{\mathrm{kz}}$ depth attenuation of vertical velocity. The earthreferenced vorticity is modeled as zero resulting in a relative vorticity of minus two times the buoy angle rates. Although the simulated motion is arbitrary, all the calculations from these assumed motions use the full nonlinear, exact equations.

In all except one of the simulations, white noise of the same variance as the actual sensors, was added to the simulated sensor measurements. As described in the Inertial Measurement Unit Calibration section, the y axis rate gyro had a small, 17-second sinusoid added to white noise. In one set of simulations, the same size sinusoid was added to white noise for this channel, to measure the inertial algorithm's response to this realistic noise.

\section{GENERAL ACCURACY RESULTS OF SIMULATIONS}

The simulations show that the inertial measurement unit and the inertial processing meet the specifications described in the Inertial Measurement Unit section, for measuring shear and vorticity. The results of two sets of such wave simulations and their system error are described. A second order statistic of wave height called significant wave height is reviewed and used to describe these two simulations. The significant wave height is the average wave height of the one-third largest waves. For a normally distributed wave field, this height is four times the square root of the variance of the sea surface (Earle and 
Bishop, 1984). In these simulations, the waves are not normally distributed, but this formula is used as a description of sea roughness. One set of $\operatorname{simulations}(\operatorname{sim} 7, \operatorname{sim} 7 f$, and $\operatorname{sim} 7 \mathrm{~s}$ ) had four waves at periods of nine, six, four and two and a half seconds; had a 1.25-meter significant wave height; and had white sensor noise only. The nine second and four second waves traveled east while the six second and two and a half second waves traveled north. In these simulations, the root mean square (rms) Euler angle error in pitch and roll was 0.25 degrees each and 1.0 degrees in heading. A second set of simulations $(\operatorname{sim} 7 \mathrm{c})$ had larger waves at periods thirteen, nine, six, and four seconds; had a 3.7-meter significant wave height; and had the more realistic $y$-axis rate gyro noise of white and sinusoidal noise. The thirteen second and six second waves traveled north while the nine second and four second waves traveled east. In the second set of simulations, the rms Euler angle error in pitch was 0.65 degrees, roll was 0.9 degrees and heading was 1.5 degrees.

The simulations section makes several references to a deployment of the vorticity measuring buoy south of Martha's Vineyard in significant swell. The wave conditions during this deployment are considered typical for open-ocean conditions. This second set of simulations had the same rms pitch and roll angles, and pitch and roll rates, as the deployment south of Martha's Vineyard with substantial swell. These simulated errors are within the specification error of three degrees.

\section{INTERMEDIATE COMPUTATIONAL REFERENCE FRAME FOR HEADING}

The effect on the accuracy of the simulated heading with and without using an intermediate, horizontal, computational reference frame was computed. Using the higher sea state of $\operatorname{sim} 7 \mathrm{c}$ with a significant wave height of 3.7 meters, the raw heading with the computed intermediate horizontal reference frame for magnetic flux had a rms error of 2.2 degrees. After complementary filtering with the heading estimate from the rate gyros, this error was reduced to 1.5 degrees. If the raw heading is computed directly from the inverse tangent of the $\mathrm{x}$ and $\mathrm{y}$ magnetic fluxes in the buoy axes, the rms heading error was 15.3 degrees. 


\section{REJECTION OF CONING MOTION}

The ability of a strapdown, inertial measurement system to not bias angle rates when undergoing a coning motion is a classic strapdown system test. A simulation was done that had two waves causing coning in the presence of two other waves. As the Coning Motion section developed, if $\psi$ is a sine wave, $\theta$ is a cosine wave, and the heading $\phi$ is zero, then a vertical rate gyro on the instrument will measure a time-average heading rate of one half the sine and cosine amplitude squared multiplied by the radian frequency (2-16). The simulation exposed the buoy to 4.8-degree coning motion, which should give a time-average, buoy-measured, vertical rotation rate of $0.0037 \mathrm{rad} / \mathrm{s}$. In the simulation, the calculated Euler heading rate rejected $95 \%$ of this bias. This resulting error in earthreferenced vorticity is below the $1 \times 10^{-2} / \mathrm{s}$ that is the measurement goal. Coning motion of a buoy built to measure this scale vorticity would, in reality, rarely cause error in excess of $1 \times 10^{-2} / \mathrm{s}$ even without compensating for it. This simulation is, however, a good verification of the inertial system and shows that coning motion is not a problem for this system.

\section{LOW FREQUENCY ERRORS}

While the inertial measurement system measures buoy attitude adequately to measure vorticity and shear, it does produce low frequency errors in calculated buoy velocity. Errors in calculated buoy velocity are summed with measured buoy relative velocity producing errors in calculated earth-referenced fluid velocities. Most of the error in computed Euler angles occurs at low frequencies. These low frequency errors in pitch and roll are on the order of a quarter degree. Pitch and roll angle errors cause errors in calculated horizontal acceleration when removing gravity from the buoy measured accelerations. These calculated horizontal accelerations are then integrated to calculate horizontal buoy and wave velocities. Integrating low frequency errors amplifies the errors.

The size of these low frequency errors in velocity can be inferred from data taken from an instrument deployment in the open ocean. The calculated power spectral densities 
of east and vertical wave velocity from a deployment South of Martha's Vineyard are shown in Fig. 2-24. The horizontal velocities below a period of 15 seconds and the vertical velocities below a period of 25 seconds have large errors. The vertical velocity estimate is much less affected by the small, low frequency errors in pitch and roll because in subtracting gravity, vertical $g$ is multiplied by the cosine of the pitch and roll angles. The pitch and roll angles rarely exceeded ten degrees. Horizontal g compensation,

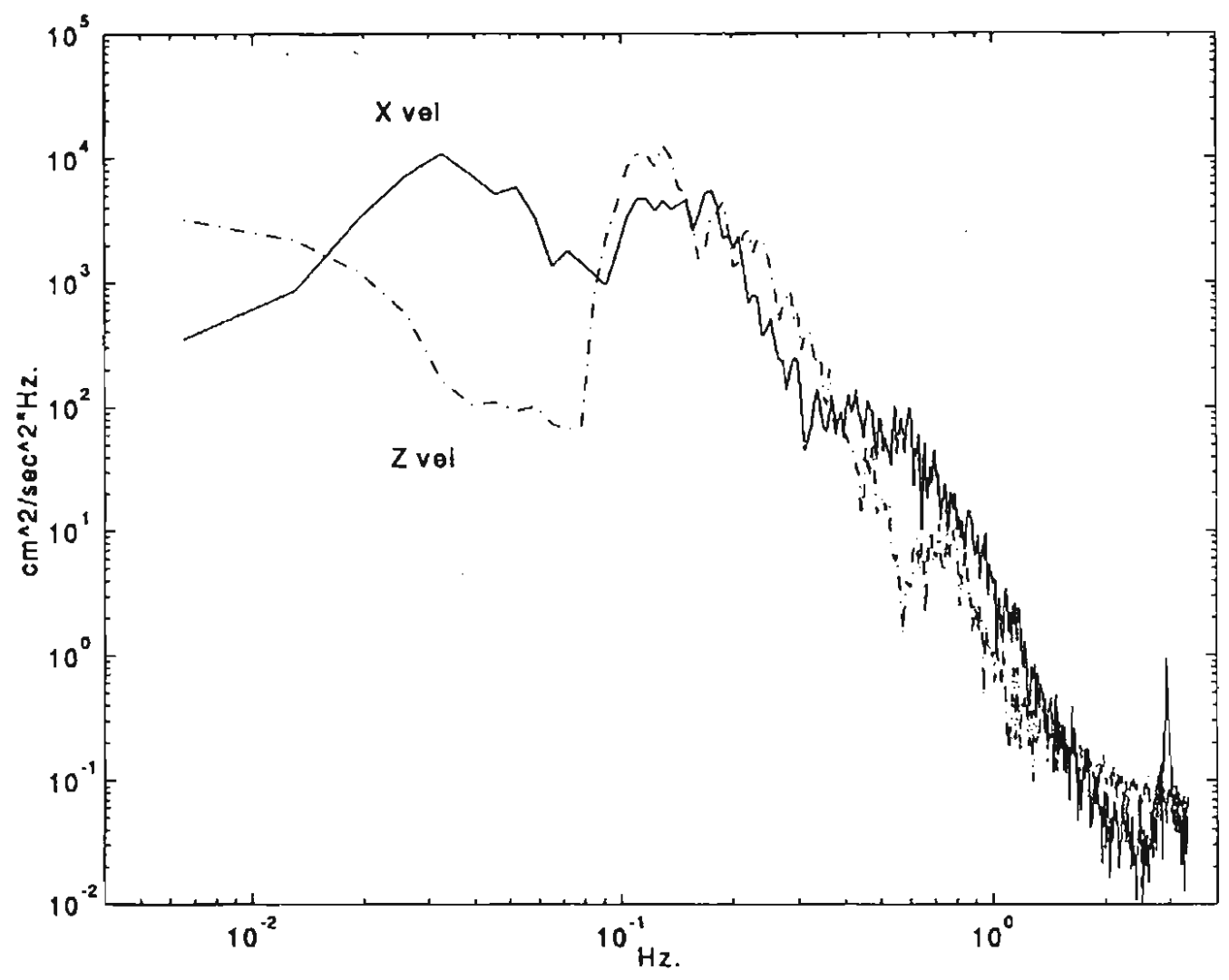

Fig. 2-24. Calculated east ( $\mathrm{X}$ vel) and vertical ( $\mathrm{Z}$ vel) velocity spectral densities of the lower sensor from deployment south of Martha's Vineyard.

however, is multiplied by the sines of these angles. The Error Propagation section showed that the Euler-angle error from the integrated-rate gyro, changed very slowly and could have slow oscillations. Pitch, roll wave-measuring buoys are not very accurate 
either at low frequencies, because these longer wavelength waves usually have small slopes.

In simulating various test algorithms while developing the inertial algorithm, it was helpful to have a single number to represent low frequency noise. None of the simulations had waves of frequency lower than a 13 second period. The statistic chosen to represent low-frequency noise was the velocity variance between the frequencies corresponding to a 30-second wave and a 15-second wave. This statistic is just the velocity spectrum integrated from $1 / 30 \mathrm{~s}$ to $1 / 15 \mathrm{~s}$. In these simulations, any energy in this band is error. Recall that in processing, the velocities are high-pass filtered with a 30 second high-pass filter so there should not be much energy below the low frequency cutoff.

Much of the inertial measurement system error comes from sensor noise. The relative contribution to error from sensor noise is shown in Fig. 2-25. This figure shows the power spectral densities of east velocity in two simulations. The solid line is the spectrum of the simulated measured velocity from $\operatorname{sim} 7$ and has a variance of $18.5 \mathrm{~cm}^{2} / \mathrm{s}^{2}$ between 30 seconds and 15 seconds. The dash-dot-dash line is the spectrum of the simulation input velocity. The dotted line is the spectrum of a two-dimensional, complementary-filtered, horizontal-velocity estimate from just the white noise from one accelerometer channel and one rate gyro channel. The velocity output was high-pass filtered and has a variance of $3.2 \mathrm{~cm}^{2} / \mathrm{s}^{2}$ between 30 seconds and 15 seconds. A perfect three dimensional algorithm with these sensors, at this sample rate, could not do better than this level. Most of the noise is from the rate gyro. The integrated accelerometer noise that is high-pass filtered, has a variance of $0.2 \mathrm{~cm}^{2} / \mathrm{s}^{2}$ in the 30 second to 15 second period band. A high-pass filtered, velocity estimate from just the rate-gyro noise has 3.6 $\mathrm{cm}^{2} / \mathrm{s}^{2}$ of variance in this band. This is more than the complementary-filtered, twodimensional model showing some of the noise reduction with complementary processing. The high spectrum values in the lowest one or two frequency bins is an artifact of Matlab's detrending and can be ignored. 


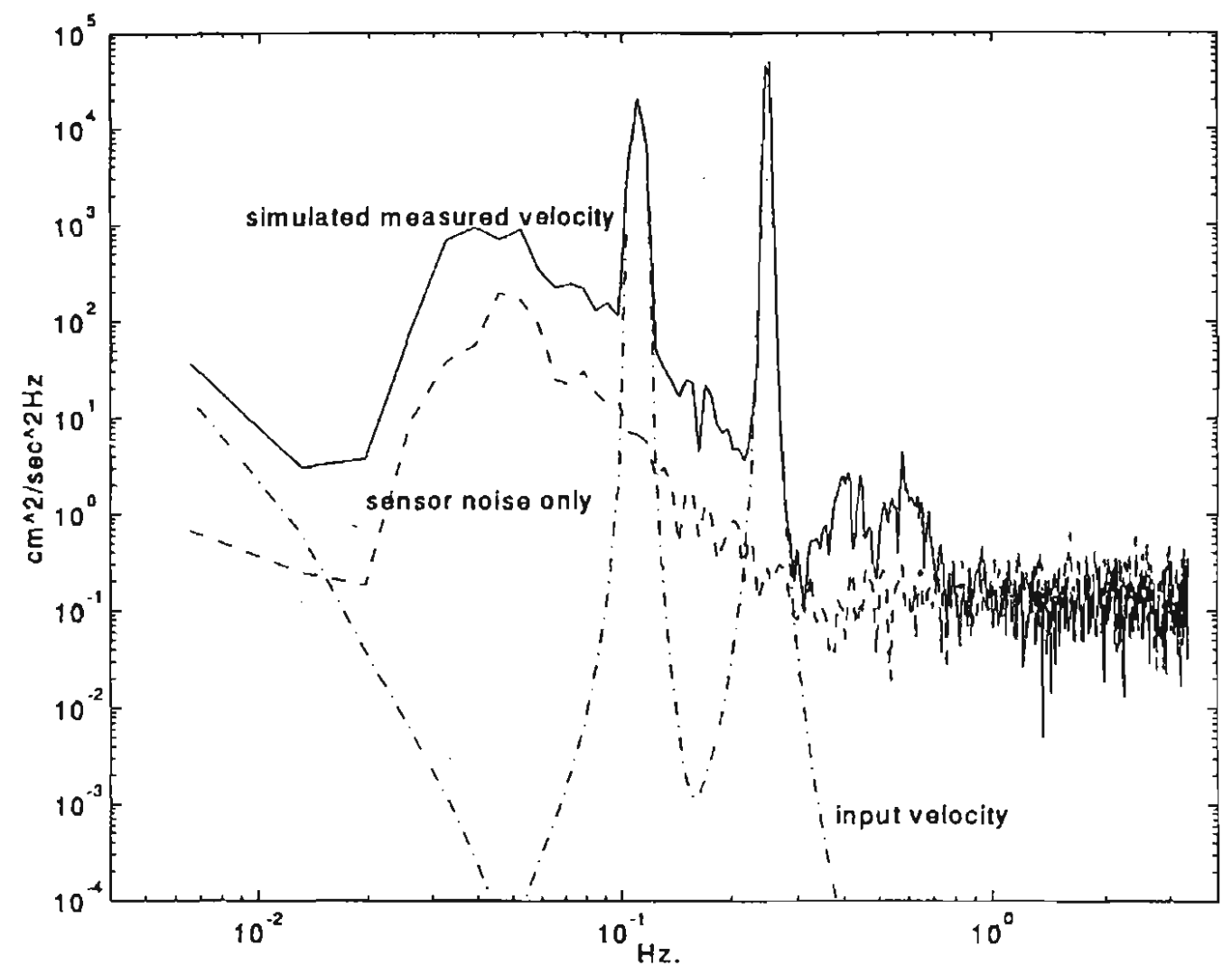

Fig. 2-25. Simulated east velocity spectra.

The noise floor in this plot above $0.4 \mathrm{~Hz}$ is primarily due to the white, rate-gyro noise. The buoy coordinate system origin is at the accelerometers which is not collocated with the measurement volumes. As described in the Data Processing section, the $\omega \times r$ term is added to the calculated velocity of the buoy coordinate origin. This adds a substantial radius multiplied by the rate gyro output, to buoy velocity and causes this noise floor in velocity. Measured vorticity spectra do not come down to the noise floor due to the rate gyro noise. Recall that twice the rate-gyro output is added to measured relative vorticity in calculating earth-referenced vorticity.

Logarithmic graphs of spectra can exaggerate the low frequency energy of a signal. In Fig. 2-25, the plot of power spectral density of horizontal velocity looks like error is very significant. However, only $4 \%$ of the velocity variance is error and $96 \%$ is the input 
velocity.

Getting back to Fig. 2-24 and the low frequency velocity variance; simulations $(\operatorname{sim} 7 c)$ with the same rms Euler angles, Euler angle rates, and the more accurate $y$-axis rate gyro noise, had the same horizontal velocity variance as the deployment south of the Vineyard. If these simulations did not have this low-frequency, velocity variance, the simulations would have been inaccurate.

\section{ALGORITHM ERROR BREAKDOWN}

The four major sources of error in a strapdown inertial algorithm are commutivity error, integration error, round-off error, and sensor lags. The least-count, analog-todigital conversion noise in this system is well below sensor noise and will not be considered further. This instrument uses a 16 bit analog-to-digital converter and the analog inputs are scaled to effectively use the converter's range. I will discuss each of these four major error terms separately in conjunction with the matrix of simulations in Table 2-4. The simulations shown in this table varied sample rates while keeping the waves constant, and also doubled the Euler angles and angle rates at the 0.15 second sample rate. For each simulation, the rms Euler angle error $(\psi, \theta$, and $\phi)$ and east velocity variance between a 30 -second period and a 15 -second period are listed.

The commutivity error results from the difference between the actual, smooth continuous rotation of an object and the discrete, sequential, orthogonal motion assumed by the Euler angle system. This error is proportional to the square of the angular displacement in each calculation step. The total error for an angular evolution is then proportional to the sampling time step and is proportional to the square of the angle rates during that evolution. For the Euler angle rates modeled, the maximum Euler angle error from commutivity over a 30 -second evolution, is 2.6 degrees. The simulations, however, show angle errors of order a quarter degree due to some canceling out of these errors in cyclic motion. Commutivity error is inherent in any strapdown inertial system and can be reduced by sampling faster. The instrument is close to its hardware speed limit, so to 
0.51 second sampling, $\operatorname{sim} 7 \mathrm{~s}$

std. angle error $\quad 0.36^{\circ} \quad 0.41^{\circ} \quad 0.45^{\circ}$

L/15

$\int_{1 / 30} P_{X v e t} d F=124 \mathrm{~cm}^{2} / \mathrm{s}^{2}$

0.15 second sampling, $\operatorname{sim} 7$

double amplitude

std. angle error $\quad 0.23^{\circ} \quad 0.27^{\circ} \quad 1^{\circ}$

1/15

$\int_{1 / 30} P_{X v e l} d F=18.5 \mathrm{~cm}^{2} / \mathrm{s}^{2}$

0.15 second sampling, sim7b

std. angle error $1^{\circ} \quad 1^{\circ} \quad 2.25^{\circ}$

1/15

$\int_{1 / 30} P_{X v e d} d F=398 \mathrm{~cm}^{2} / \mathrm{s}^{2}$

0.05 second sampling, $\operatorname{sim} 7 \mathrm{f}$

std. angle error $\quad 0.17^{\circ} \quad 0.19^{\circ} \quad 3.1^{\circ}$

I/15

$\int_{1 / 30} P_{X v e l} d F=5.8 \mathrm{~cm}^{2} / \mathrm{s}^{2}$

0.15 second sampling $2 \mathrm{D}$

accelerometer and rate gyro noise into complementary filter

velocity high passed

1/15

$\int_{1 / 30} P_{X v e l} d F=3.2 \mathrm{~cm}^{2} / \mathrm{s}^{2}$

Table 2-4. Array of simulations used to infer error terms.

sample significantly faster would require changes in hardware. Referring to Table 2-4, $\operatorname{sim} 7 \mathrm{~b}$ is the same as $\operatorname{sim} 7$ but with all waves, Euler angles and Euler angle rates doubled. The angle errors in the doubled simulation are quadrupled, consistent with commutivity error dominating algorithm error. The velocity variance increased by more than sixteen due to doubling the angle rates. In $\operatorname{sim} 7$, over one-sixth of the velocity variance is accounted for by the two-dimensional sensor noise, sim5d.

The inertial algorithm uses trapezoidal nule integration. The integration error 
during an evolution should be proportional to the time step squared and proportional to the second derivative of the quantity integrated (Thomas, 1972). The angle error accrued in 30 seconds of processing with the simulated angle rates is of order $0.2^{\circ}$. In cyclic motions, however, much of this error cancels out and this error term is much smaller than commutivity error. In one simulation, I tested second- and third-order Runge Kutta integration but the output error was not measurably better, so trapezoidal integration was kept.

Round-off error in double-precision, floating point math can occur if processing is not well conditioned. The complementary filter processing used is not well conditioned because the crossover frequency is a very small fraction of the Nyquist frequency. Referring to the simulations in Table 4, when the sampling rate is slowed from 0.15 seconds to 0.51 seconds, the angle error is increased a little and the horizontal velocity variance is increased much more. This increase in error is largely due to commutivity error. As the sampling rate is increased to 0.05 seconds, however, the error does not drop as much as would be expected if commutivity error still dominated error at the faster sample rate. Both the commutivity error and the integration error should drop significantly at this faster sampling rate. To explain why the faster sample rates do not decrease error more, the complementary filters were tested. Normal random vectors, of standard deviation equal to one, were complementary filtered with the filters from the three sampling rates. The slow sampling rate filter output had a standard deviation of error of $1 \times 10^{-11}$. The medium sampling rate filter for the 0.15 second sampling, produced an output with a standard deviation of error of $1 \times 10^{-10}$, which is still small but much larger than the least count scale of $2.2 \times 10^{-16}$. When the same random vector is filtered by the 0.05 second filter the standard deviation of error jumped to 0.024 . The pole zero diagram of the high- and low-pass filters for the 0.05 second sampling is shown in Fig. 2-26. When the poles and zeros are this close together, even double precision math can have errors. If the hardware allowed it, sampling at 0.05 second would have less error than sampling at 0.15 seconds; but if sampling rates were desired to be significantly faster than 0.05 seconds, this complementary-filter conditioning would become a problem. The 
high-pass part of a complementary filter cannot be downsampled. If significantly faster sampling was done, some other processing algorithm would have to be considered.

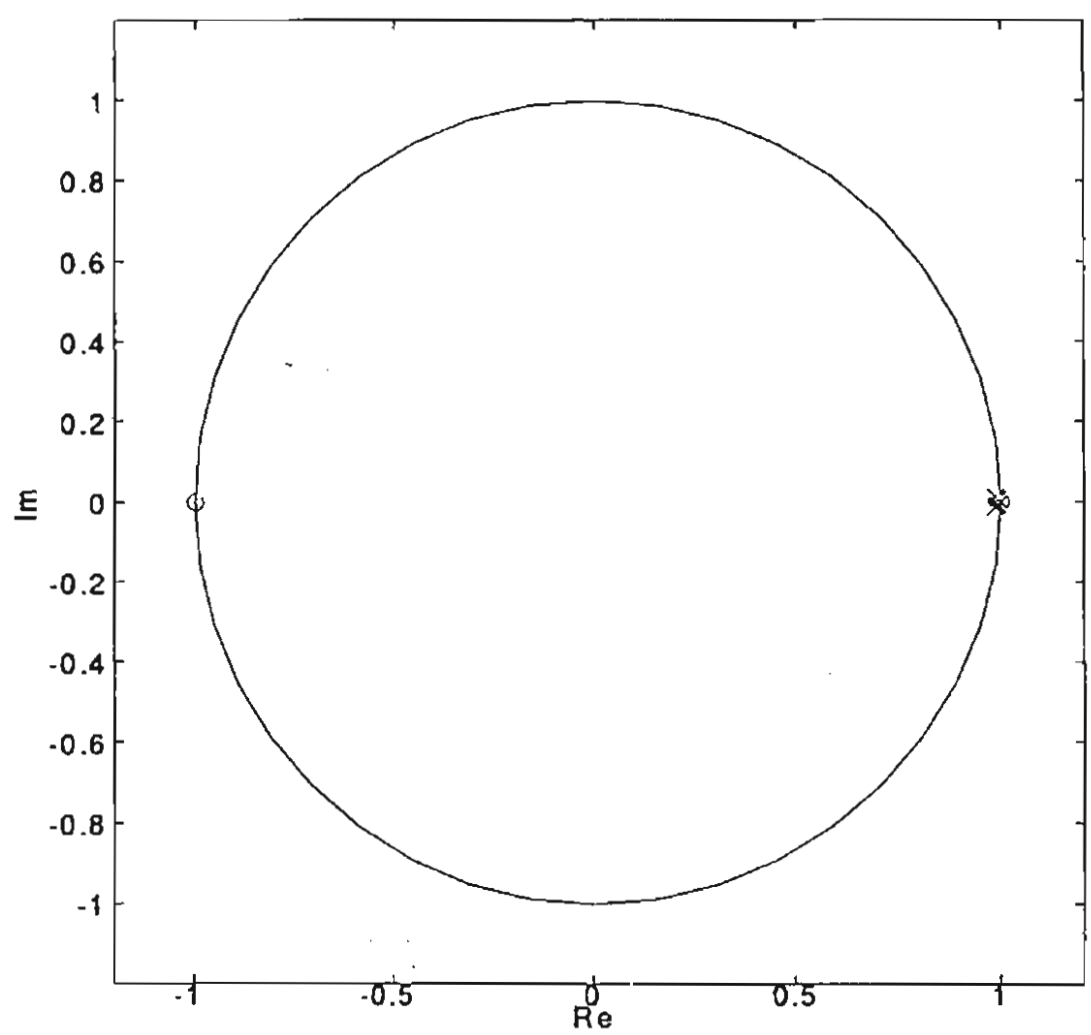

Fig. 2-26. Pole-zero plot for 0.05 second sample complementary filter

The electronics do not have a simultaneous sample and hold, so error from sensor lags and nonsimultaneous sampling have to be considered. The velocity and analog sampling in each time step is done in under 40 milliseconds. The analog inertial sensors are anti-alias filtered by a single-pole filter and at the frequencies of interest, the filter phase lag is close to a partial-time-step delay. Sampling the analog, anti-alias filtered, inertial sensors after the velocity measurements, and considering all samples to have been taken at the same time, largely cancels out the anti-alias filter lag. Measurements of the phase of the cross spectrum between sensor-referenced vorticity and its corresponding rate gyro signal, show a small lead for the analog measurements. 
To estimate the possible errors resulting from a partial period sensor lag, a simulation was run with a full period lag. The sensor lag error from a fractional period lag should be at most that fraction multiplied by the sensor lag error from the full period lag simulation. The simulation that delayed the analog measurement a whole time step increased errors over simulations with no lag. The standard deviation of pitch and roll angle error increased from 0.25 degrees to 0.65 degrees, heading error increased from 1.4 degrees to 1.6 degrees, and the low frequency velocity variance increased by $1 \mathrm{~cm}^{2} / \mathrm{s}^{2}$. The maximum actual sensor lag is a quarter of a time step. The actual sensor lag errors are at most 0.1 degrees in pitch, roll, or heading and $0.25 \mathrm{~cm}^{2} / \mathrm{s}^{2}$ in low frequency velocity variance.

\section{SIMPLER INERTIAL ALGORITHMS}

Most oceanographic instruments are sample rate limited. Most oceanographic instruments are battery powered requiring slow, low power electronics. Sample rates are usually limited by the slow electronics or the storage space required for the months of data that a typical oceanographic instrument is deployed. In strapdown inertial processing, faster sample speed for the same algorithm complexity gives better accuracy. This instrument development was hardware limited in speed and sample rate requiring, by oceanographic standards, a complex inertial algorithm. In a more general instrument development, the designer has to tradeoff algorithm complexity and sample rate.

Simpler inertial algorithms were simulated to evaluate the tradeoff between algorithm complexity and accuracy. Three of the simulations are summarized in Table 25. The first simulation listed is the same $\operatorname{sim} 7$ as in Table 2-4 for comparison. The second simulation listed, sim7l, used a linear, Euler-angle update matrix and the same complementary filtering as $\operatorname{sim} 7$. The standard deviations of angle error increased for pitch and roll from $0.23^{\circ}$ and $0.27^{\circ}$ to $0.40^{\circ}$ and $0.47^{\circ}$ while the heading error standard deviation stayed the same at one degree. The low frequency velocity variance, however, jumped from $18.5 \mathrm{~cm}^{2} / \mathrm{s}^{2}$ to $63 \mathrm{~cm}^{2} / \mathrm{s}^{2}$. If only buoy attitude was desired from a strapdown inertial system, this simpler algorithm might be adequate. 
A simulation was run, but not listed, that took out the $\cos \psi$ term in computing the slow estimate of $\theta$. For a 3,2,1 Euler angle system, the slow estimate of $\theta$ is given by $\tan ^{-1} \frac{\ddot{x} \cos \psi}{\ddot{z}}$. In these simulations, the pitch and roll angles never exceeded $15^{\circ}$ so the simulation results were the same with and without the $\cos \psi$ term. This term was left in because theoretically it should be there and if the pitch and roll angles were greater, should improve accuracy.

$$
\begin{array}{lll}
\text { Full nonlinear processing } & \text { sim7 } \\
\text { std angle error } \quad 0.23^{\circ} & 0.27^{\circ} & 1.0^{\circ} \\
1 / 15 & & \\
\int_{1 / 30} P_{X v e} d F=18.5 \mathrm{~cm}^{2} / \mathrm{s}^{2} &
\end{array}
$$

Linear Euler-angle update matrix $\operatorname{sim} 71$

std angle error $\quad 0.40^{\circ} \quad 0.47^{\circ} \quad 1.0^{\circ}$

$1 / 15$

$\int_{1 / 30} P_{X v e} d F=63 \mathrm{~cm}^{2} / \mathrm{s}^{2}$

Linear update matix, first-order, causal complementary filter

std angle error $\quad 0.54^{\circ} \quad 0.59^{\circ} \quad 8.04^{\circ}$

$$
\int_{1 / 30}^{1 / 15} P_{X v e f} d F=80 \mathrm{~cm}^{2} / \mathrm{s}^{2}
$$

Table 2-5. Simulations of simpler inertial algorithms

The last simulation listed in Table 2-5 is of an algorithm with a linear Euler angle update algorithm and first-order causal high- and low-pass complementary filters. Its pitch and roll error standard deviations increased to $0.54^{\circ}$ and $0.59^{\circ}$ while its heading error jumped to over $8^{\circ}$. This simpler algorithm gives a good vertical-velocity spectrum but has unacceptable heading errors. If in a future instrument development, in-situ processing of attitude was desired, one of these simpler algorithms sampled and processed at a faster rate would have to be considered. 
These inertial simulations show that this inertial system meets the specifications for measuring shear and vorticity. The simulations of estimated error in measured vorticity due to inertial system errors, predict mean errors of $5 \times 10^{-5} / \mathrm{s}$ and standard deviation of error of $4 \times 10^{-3} / \mathrm{s}$. Most of the time varying error is due to rate gyro noise. The measured standard deviation of vorticity, in an open ocean deployment, was fifteen times this magnitude showing that most of the measured vorticity variance is due to wakes and turbulence in the flow and not IMU errors. If a system is desired to measure horizontal velocity spectra to periods longer than 15 seconds, faster sampling and/or better rate gyros are needed. 


\section{CHAPTER 3. SENSOR}

\section{PERFORMANCE}

This chapter describes the vorticity sensor's major sources of error and also describes measurements of the instrument's accuracy. The inertial system simulations of the last chapter showed that errors in instantaneous and mean vorticity resulting from inertial system errors was much less than $1 \times 10^{-2}$ per second. Errors from wakes of the sensor advecting through the sensing volume are the major error sources. This chapter models in constant flow: potential flow disturbance due to the transducer pods, wake error due to the center stalk wake, wake error due to the transducer pods, circulation disturbance from lift on the center stalk, and sound speed changes. This chapter then shows through measurements that: electronic noise and bias are much less than $1 \times 10^{-2}$ per second, symmetry of the sensor design succeeded in keeping biases in constant flow to less than $1 \times 10^{-2}$ per second, down-wave and vertical vorticity biases in open-ocean waves are less than $1 \times 10^{-2}$ per second, and cross-wave vorticity bias in open-ocean waves is about $1 \times 10^{-2}$ per second and scales with rms, sensor-relative velocity.

\section{A. MODELING SENSOR ERRORS}

This section develops models of sensor flow disturbance and error. Potential flow is used to model flow around the transducer pods and predicts a reduced gain (that can be calibrated) but no bias or noise. Wake models are developed to describe bias in measured circulation due to the center stalk and the transducer pods. The circulation bias that would result from lift off the center stalk is predicted and the result is used to justify not streamlining the cylinders in the sensor.

The potential flow solution of flow around spheres can be used to gain some insight into flow errors. The geometry and coordinate system modeled are shown in Fig. 3-1. This model only considers the spheres; it ignores pod arms, and it assumes that the spheres are moving relative to a still fluid. In this model, $L$ is the distance between 


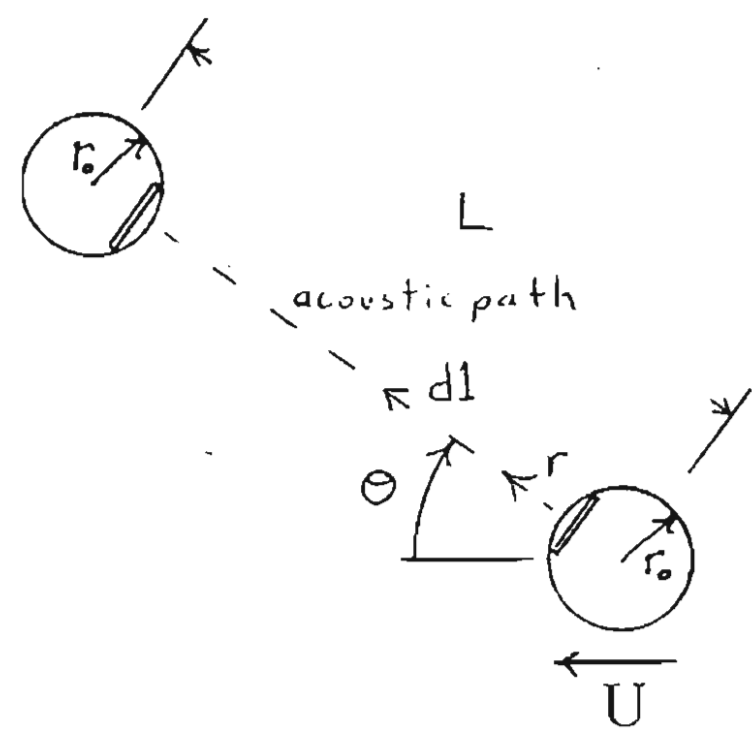

Fig. 3-1. Geometry of potential flow model.

spheres, $\theta$ is the angle between the flow and the acoustic path, $r$ is the radius from the sphere center, and $r_{o}$ is the sphere radius. The sphere diameter is much smaller than the distance between the spheres so there is little interaction between the sphere disturbance fields. This model calculates the line integral of velocity disturbance from one sphere over an acoustic length $\mathrm{L}$, and doubles it. The flow potential field $\Phi$ for the disturbance field from one sphere is given by equation (3-1). The radial velocity line integral for the

$$
\Phi=-\frac{1}{2}\left(\frac{U r_{o}^{3}}{r^{2}}\right) \cos \theta
$$

disturbance from one sphere is then equation (3-2). The potential flow disturbance retards radial flow both upstream and downstream of the sphere causing acoustic velocity measurement to under-report velocity. To calculate the velocity speed error, the radial 


$$
\int_{r_{0}}^{L} \frac{U r_{o}^{3}}{r^{3}} \cos \theta d r=\frac{U r_{0} \cos \theta}{2}
$$

velocity line integral is doubled and divided by the line integral of undisturbed velocity (33). For the 15 -centimeter path vorticity meter, the velocity error is $0.0741 \cos (\theta) * U$ or

$$
\frac{\text { velocity error }}{\text { velocity }}=\frac{d}{2 L} \cos \theta
$$

about $7.5 \%$ of the flow, which is a significant error. However, because of the $\cos \theta$ term, this error just changes the velocity-calibration constant of the sensor. Potential flow theory predicts that if the flow upstream of the sensor is irrotational, the flow around the vorticity sensor will stay irrotational. If the flow around the sensor is irrotational, there will be zero measured circulation, ie, no vorticity bias in irrotational flow. If the flow upstream of the sensor has a small amount of shear compared to the shears of the sphere's disturbance fields, the above model predicts under-reporting of the shear or vorticity by the same ratio that velocity is under-reported. Calibrating the velocity gain should also calibrate the vorticity gain. This potential flow model should apply well upstream of a sphere, but because of boundary layer separation, the model is not realistic downstream of a sphere.

Models were developed for the circulation error resulting from wakes from the center stalk and transducer pods. Downstream of bluff bodies, wakes resulting from boundary layer separation over bluff bodies disturb the flow more than the potential flow solution. Fig. 3-2 represents the cross sections of two vorticity meters, showing the center stalk in the middle of a square of transducer pods. The top half of the figure shows a circulation square path in the worst direction for velocity-path-wake errors. As explained in the Mechanical Design Overview section, the sensor was designed with symmetry to minimize error when measuring circulation or vorticity. The lower half of the figure shows the same sensor at an unsymmetrical angle of attack. The wake errors on paths 2 


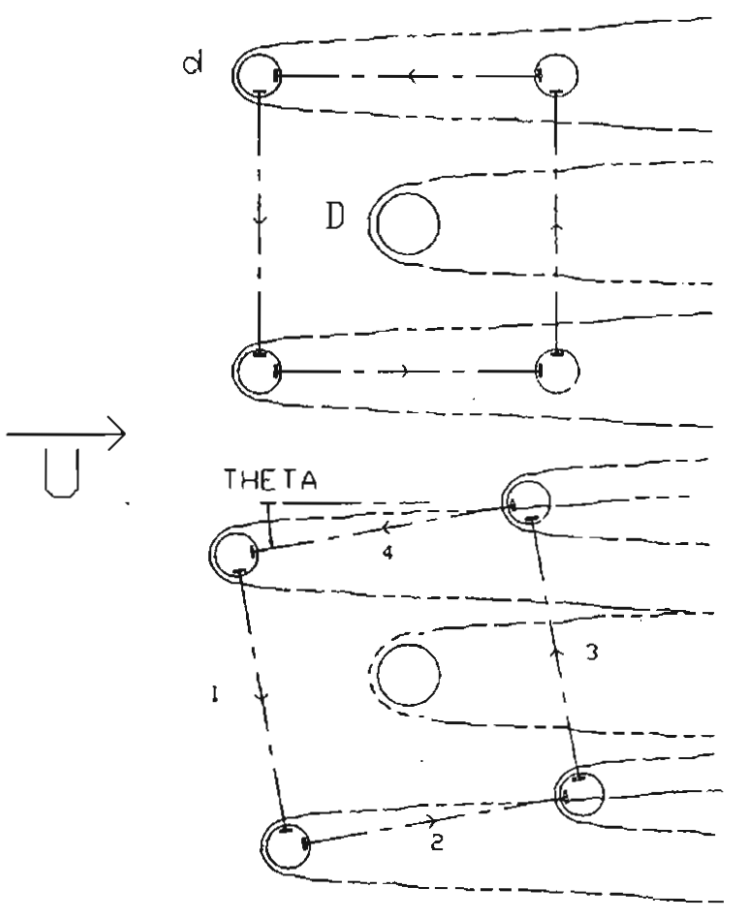

Fig. 3-2. Geometry of wake error models.

and 4 are the largest and still cancel in measuring circulation. The wake errors in paths $I$ and 3, however, do not cancel. Two models were developed to estimate circulation error resulting from path wakes from the center tube and from a sphere.

The first model developed was for the two-dimensional wake of the center stalk. The model assumes the far-field, two-dimensional turbulent wake described in Schlichting, (1979). The model geometry is shown in Fig. 3-3 and assumes an entrainment relation given by equation (3-4). In this equation, $b$ is the wake width, $x$ is the distance

$$
\frac{d b}{d x}-\beta \frac{u_{1}}{U_{\infty}}
$$

downstream, $\beta$ is the entrainment constant, $U_{-}$is the free stream velocity, and $u_{1}$ is the 


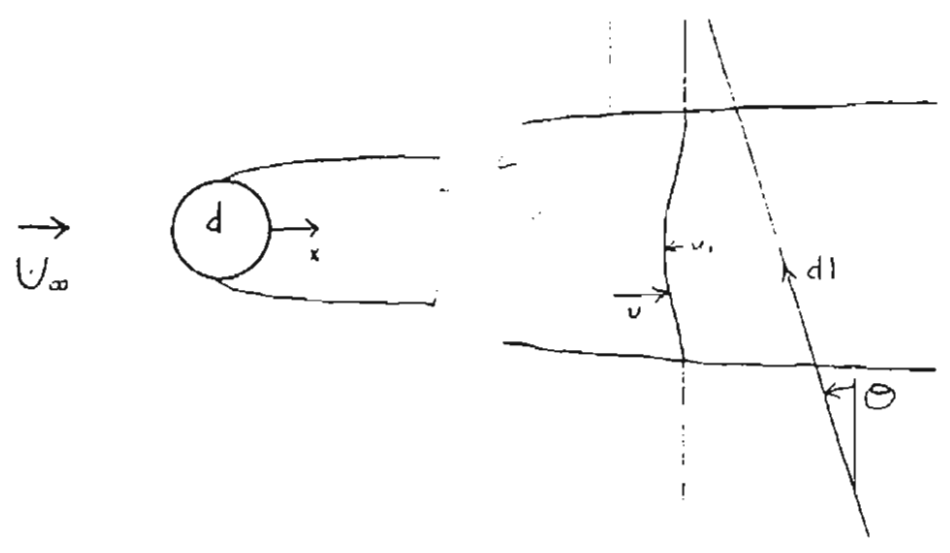

Fig. 3-3. Geometry of two- and three-dimensional models of wake circulation disturbance. The two-dimensional model stalk diameter is $\mathrm{D}$ and the threedimensional model pod diameter is $\mathrm{d}$.

wake velocity difference from the undisturbed fluid. The momentum deficit of the wake is given by equation (3-5) and is constant with distance downstream. In this equation, $u$ is

$$
\begin{gathered}
J=\rho \int_{A} u\left(U_{\infty}-u\right) d A \\
J=-\rho U_{\infty} \int u_{1} d A
\end{gathered}
$$

the velocity in the wake, $J$ is the drag per unit length on the center stalk, $\rho$ is density, and the area over which the wake is integrated is perpendicular to the wake and downstream of the strut. The wake width scales as equation (3-6) and the wake velocity scales as

$$
b-\sqrt{\beta x C_{D} D}
$$

equation (3-7).

$$
u_{1} \sim U_{\infty} \sqrt{\frac{C_{D} D}{\beta x}}
$$


The circulation disturbance through the wake is the line integral of velocity along a circulation path and is given by equations (3-8). Only the line integral through the wake

$$
\begin{aligned}
& =\int u \cdot d l \\
\Delta \text { circulation } & =\int u_{1} \sin \theta d l \\
& =\int_{A} u_{1} \tan \theta d y
\end{aligned}
$$

contributes to circulation disturbance because outside the wake, even in the areas where the flow is accelerated, the flow is irrotational. Using the scaling expressions in equation (3-8) gives equation (3-9) where $K$ is an as yet undetermined constant. The far field

$$
\text { scirculation }=K u_{1} b
$$

momentum deficit and circulation disturbance equations are both linear in $u_{1}$ the velocity anomaly and $b$ the wake width, which means that the actual distribution of $u$, is unimportant. For this model a top hat distribution is assumed where $u_{l}$ is constant over the wake width. The undetermined constant, $K$, can be determined from the momentum equation (3-5) to be one half. The result for the circulation and vorticity disturbance is given by equation (3-10). Line averaging across a two-dimensional wake in the far field,

$$
\begin{aligned}
& \Delta \text { circulation }=\frac{1}{2} U_{\infty} C_{D} D \tan \theta \\
& \Delta \text { vorticity }=\frac{1}{2} \frac{U_{\infty} C_{D} D \tan \theta}{L^{2}}
\end{aligned}
$$

is constant with distance downstream, is independent of the velocity distribution in the wake, and is independent of $\beta$ the entrainment constant. The measured circulation depends on the circulation path in rotational flows.

This bias correction from the center-stalk wake model was applied to data taken 
with the sensor towed through still water. The geometry of this model application is shown in Fig. 3-4. The circulation path squares look like the figure when viewed parallel

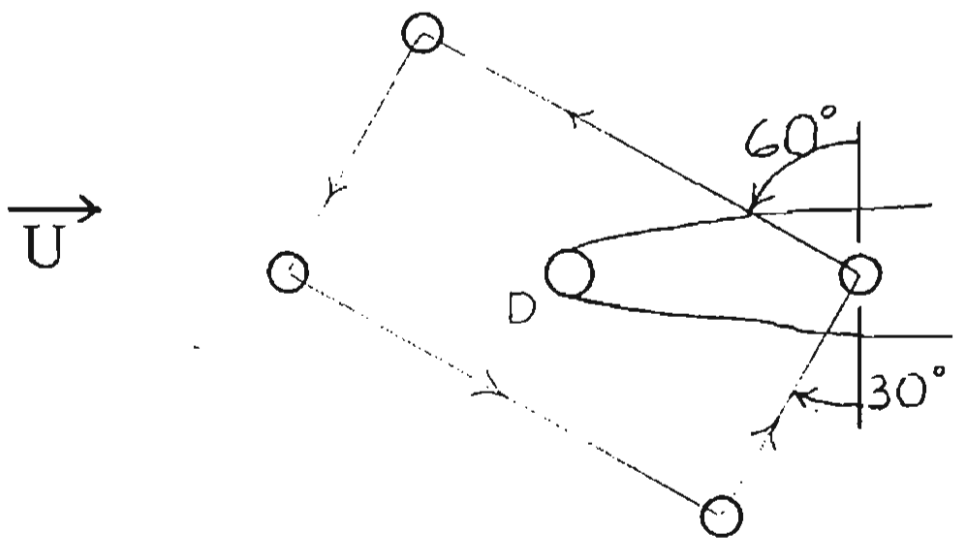

Fig. 3-4. Center stalk model used on tow tank data

to the center stalk. Half of the wake is assumed to flow on each side of the downstream pod and the resulting measured wake circulation and vorticity errors are calculated by equation (3-11). Vorticity vectors perpendicular to each circulation path square are

$$
\begin{gathered}
\text { circulation error } \doteq U_{\infty} \frac{D}{4} \tan \left(60^{\circ}\right)-U_{\infty} \frac{D}{4} \tan \left(30^{\circ}\right) \\
\text { vorticity error } \doteq \frac{0.288 U_{\infty} D}{L^{2}}
\end{gathered}
$$

labeled avort, bvort, and cvort. The vorticity measurement avort was measured with its circulation path as shown in the Fig. 3-4 and cvort was measured with a circulation path that was the mirror image of the figure. In the tow tests, the vorticity was rotated into axes parallel to the tank yvort, across the tank xvort, and vertical zvort. The error in these coordinates was then given by equation (3-12). This is a simple, far-field model 


$$
\begin{gathered}
x \text { ort }=\frac{1}{\sqrt{2}} \times \text { avort }-\frac{1}{\sqrt{2}} \times \text { cvort } \\
\text { xvort } \doteq \sqrt{2} \times \frac{0.288 U_{\infty} D}{L^{2}}
\end{gathered}
$$

being applied in the near field. This simple model's assumption of the wake flowing on each side of the pod and the pod not effecting the measured circulation is unrealistic.

Some of the stalk wake flows around the downstream pod in the dimension parallel to the stalk and out of the plane of this two-dimensional model. The actual correction applied to measurements from the 45 -centimeter path vorticity sensor was $68.7 \%$ of the above model, and in constant flow accounted for $89 \%$ of the measured bias.

The second model developed was for the three-dimensional wake of the transducer pods. The geometry of the model is shown by Fig. 3-3 again, using $d$ for the pod diameter. The entrainment model used was equation (3-4) as in the two-dimensional wake model. The width is proportional to the distance downstream to the one-third (3-13) and

$$
\begin{aligned}
& b \sim\left(\beta C_{D} \frac{\pi d^{2}}{4} x\right)^{\frac{1}{3}} \\
& \frac{u_{1}}{U_{\infty}}-\left(\frac{C_{D} \frac{\pi d^{2}}{4}}{\beta^{2} x^{2}}\right)^{\frac{1}{3}}
\end{aligned}
$$

the wake relative velocity is proportional to the distance downstream to the minus twothirds. The measured wake circulation error was then given by equation (3-14) for an

$$
\Delta \text { irculation } \sim U_{\infty} \frac{C_{D}^{\frac{2}{3}} \frac{\pi}{4} d^{\frac{4}{3}}}{\beta^{\frac{1}{3}} x^{\frac{1}{3}}} \tan \theta
$$


acoustic path through the wake center. The measured vorticity error assuming the distance downstream is $L$, is given by equation (3-15). This result shows that the

$$
\Delta \text { vorticity } \sim \frac{U}{L}\left(\frac{d}{L}\right)^{\frac{4}{3}} \tan \theta
$$

measured circulation or vorticity error changes with distance downstream, depends on the entrainment constant $\beta$, and depends on the velocity distribution in the wake. Although applying this far-field wake model to the relatively near-field wake in this sensor application is tenuous, this solution's scaling of error with pod size and path length provides a framework in which measurements of error can be compared.

The circulation bias that would result from lift from the center stalk will now be calculated to justify not streamlining the stalk. If the center stalk were not round, there could have been lift off the stalk and consequent lift-related circulation. The geometry of a lift-related circulation model is shown in Fig. 3-5. The relation between lift and

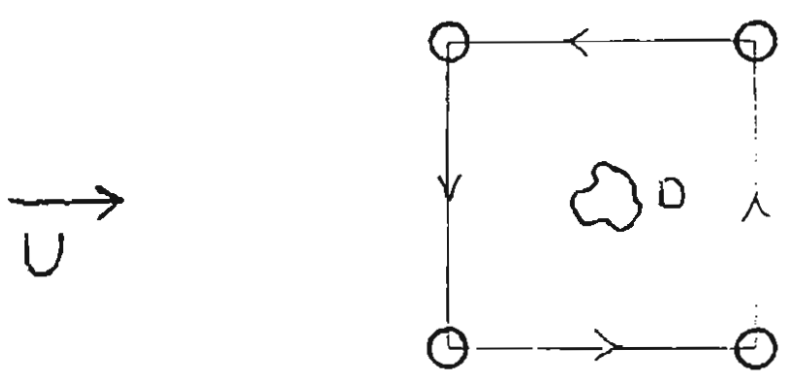

Fig. 3-5. Geometry of lift related circulation model. 
circulation is given by equation $(3-16)$ where $C_{L}$ is the coefficient of lift, $\Gamma$ is circulation,

$$
\begin{gathered}
\Gamma=\int u_{i} d x_{i} \\
L=\rho U \Gamma=\frac{1}{2} \rho U^{2} D C_{L}
\end{gathered}
$$

and $\rho$ is density. The change in circulation and vorticity is given by equations $(3-17)$. As

$$
\begin{aligned}
\Delta \text { circulation } & =0.5 U D C_{L} \\
\Delta \text { vorticity } & =\frac{0.5 U D C_{L}}{L^{2}}
\end{aligned}
$$

an example, with the dimensions of the 45 -centimeter path vorticity sensor if the coefficient of lift was one (a reasonable lift coefficient for a streamlined strut) and the constant current was one meter per second, the lift-related vorticity would be 0.086 per second. If the center stalk was streamlined and had an angle of attack relative to the flow, lift caused by asymmetrical boundary layer separation off the stalk would cause significant bias. Rather than trying to deal with this bias, the center stalk was kept round and as small as structurally sound, and should have zero time-average lift.

\section{B. MEASUREMENTS OF PERFORMANCE}

This section measures electronic noise and bias in vorticity to show that they are much less than $1 \times 10^{-2} / \mathrm{s}$. Sound speed changes in gain are predicted. Bias and noise in constant flow are measured to be less than $1 \times 10^{-2} / \mathrm{s}$. Biases in simulated wave flow are measured to show that down-wave and vertical vorticity biases are less than $1 \times 10^{-2} / \mathrm{s}$ and that cross-wave bias scales with rms relative velocity. Measurements in a wave tank and in the ocean are used to show that vorticity-meter bias is insensitive to heading. A deployment with significant swell and negligible wind stress will be described to show that cross-wave vorticity bias in open-ocean waves is about $1 \times 10^{-2} / \mathrm{s}$. Vorticity meter rejection of waves is measured to show how effective the vorticity measurement is at 
removing irrotational motion from its measurements.

\section{ELECTRONIC NOISE}

Flow distortion due to the instrument being in the water dominates the electronic instrument noise and bias in all but the most still flows. The two significant sources of electrical error are electronic noise and zero drift. Current measurements made with a 15centimeter path sensor are shown in Fig. 3-6. The top graph is measured velocity in a still
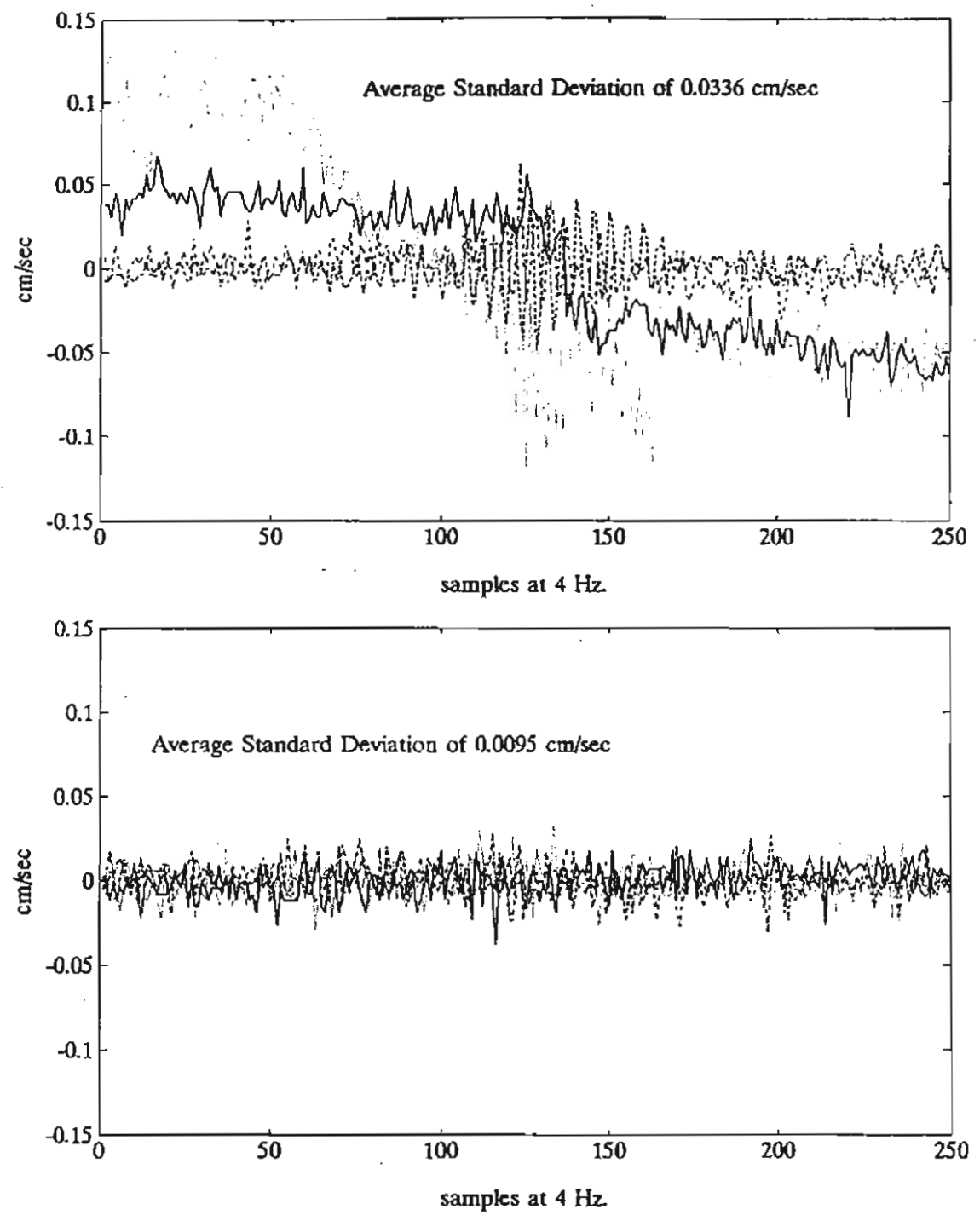

Fig. 3-6. Current measurement in a bucket. The top graph shows typical bucket convective water velocities summed with electronic noise and the bottom graph plots the measured velocities in a gel, that is still, leaving electronic noise. 
bucket of water and the lower graph is measured velocity in a gel of carrageenin, to stop convective flow. The electronic noise of this measurement has a standard deviation of $0.0095 \mathrm{~cm} / \mathrm{s}$. Normal convective velocities in a still bucket are typically 2 to $5 \mathrm{~mm} / \mathrm{s}$.

\section{ELECTRONIC ZERO DRIFT}

The zero of this instrument can change, by as much as $0.3 \mathrm{~cm} / \mathrm{s}$, if the sensor cables are moved or reconnected, causing a bias. To make accurate measurements with this instrument requires calibrating the zero flow in a bucket of still water each time the sensors are reconnected. These "bucket zeros" have to be performed carefully, avoiding differential bucket heating and averaged for many hours. Typical "bucket zeros" of velocity before and after a deployment, vary by less than a millimeter per second on each acoustic path. Because the cables are under atmospheric pressure all the time, the instrument zero does not change with instrument depth. If the cables were exposed to hydrostatic pressure, the resulting compression would change the cable capacitance and could change the instrument's zero. To put these zero changes in perspective, typical propeller current meters do not turn until flows reach one centimeter per second. Except in very still conditions electrical instrument errors can be ignored.

\section{SOUND SPEED CHANGES}

If the sound speed in water changes due to changes in temperature or salinity, and if this local speed of sound in water is not used to compute the water velocity from the differential sound travel time (2-2), an error in sensor gain will result. This gain error will be $\left(c_{a}^{2}-c_{r}^{2}\right) / c_{r}^{2}$ where $c_{a}$ is the assumed speed of sound and $c_{r}$ is the actual speed of sound. A one degree centigrade difference in temperature can result in a $0.6 \%$ error in gain. This potential source of error can be avoided by using the local sound speed.

\section{THERMISTOR TIME RESPONSE}

The thermistor time constant was measured by plunging the instrument into cool water from warm air and measuring the time delay for the measured temperature to reach $63 \%$ of the final temperature change. This time constant model assumes a first order 
thermal system. The time constant was effected by the relative flow velocities of the water, and varied from 12 seconds to 25 seconds. The thermal response time is long because the thermistors were mounted inside the fairly thick wall, stainless steel tubing. The purpose of the thermistors was to measure time average thermal stratification. The thermistor time constant was sacrificed for protection.

\section{BIAS IN CONSTANT FLOW}

\section{WAKE ERROR CANCELLATION}

Wake error cancellation was measured to evaluate the effectiveness of the sensor design, in reducing bias in constant flow. Wake error cancellation with a square circulation path is shown in Fig. 3-7. The 15-centimeter path sensor was towed with two

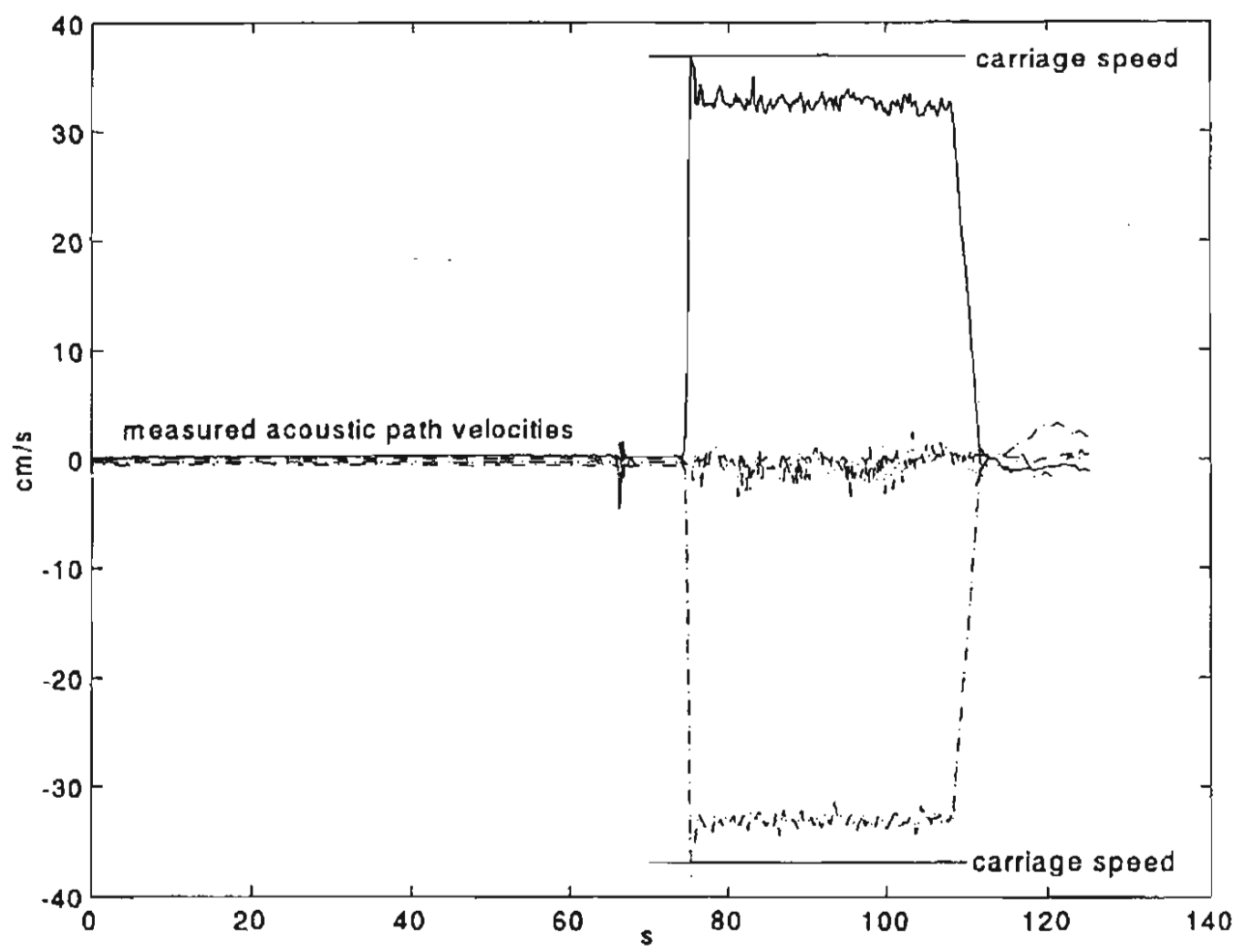

Fig. 3-7. Measured path velocities around circulation-path square while sensor was towed through still water. 
of its acoustic paths parallel to the carriage velocity, the worst orientation for measuring velocity. The acoustic paths parallel to the flow measured about $10 \%$ less than the free stream velocity, but these two decrements were the same to within $1 \%$ of the carriage velocity. The symmetry of the sensor cancels $90 \%$ of the error from transducer pod wakes. Lines were drawn at the carriage velocity and show initial velocity measurements with no or reduced wake decrement. Until the sensor has moved a path length, there is still undisturbed fluid in at least part of the acoustic path. In a wave field, water is constantly accelerating in different directions so constant-flow sensor calibrations cannot automatically be used.

The three-axis vorticity sensor also has acoustic paths that form triangles so vorticity bias in constant flow for a triangular path can be computed. For a tow at 0.37 $\mathrm{m} / \mathrm{s}$, the vorticity bias for triangle circulation paths on the top and bottom of the sensor is $0.53 / \mathrm{s}$, while the bias for the square path is $0.027 / \mathrm{s}$, Fig. 3-8. The vorticity bias of the triangle was twenty times that of the square because of the $90 \%$ error cancellation of the square and because the area of the triangle was half the area of the square path. While this sensor greatly reduces vorticity bias in constant flow by designing the acoustic circulation paths to be symmetric with sensor wakes, it does not reduce the intensity or size of the sensor wakes. In a wave field, these strong wakes left by the sensor can advect back into the measurement volume; this resulting error will be treated separately in the bias in wave flow section. 


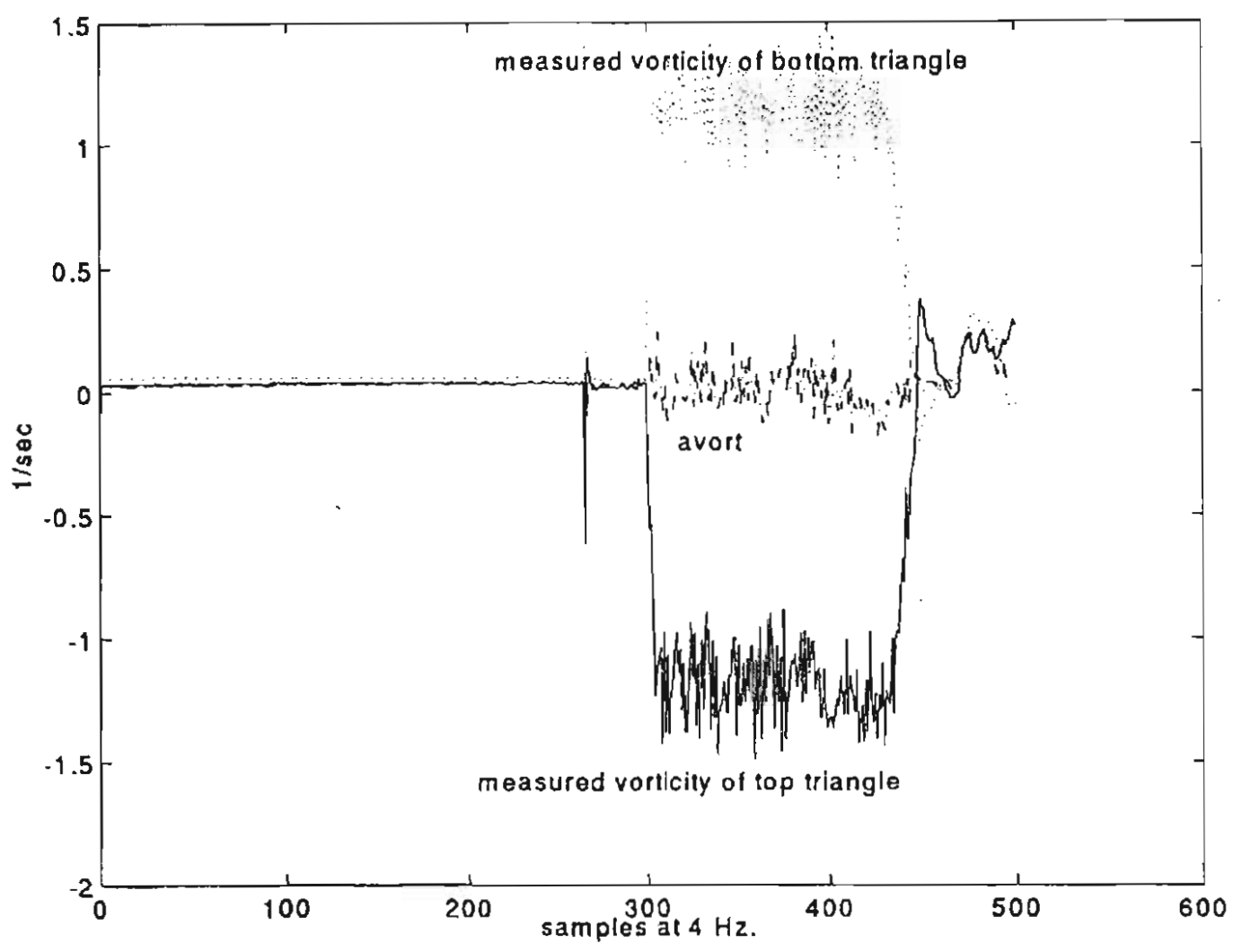

Fig. 3-8. Vorticity bias in constant flow for triangular and square (avort) circulation paths. Tow speed $0.37 \mathrm{~m} / \mathrm{s}$.

\section{MEASURED BIAS IN CONSTANT FLOW}

Vorticity bias in constant flow was measured by towing both 15 -centimeter path and 45-centimeter path vorticity sensors in tanks of still water. Uncorrected average vorticities of both sensors for different tow carriage speeds are shown in Fig. 3-9. For the 45-centimeter path sensor, the $\mathrm{x}$ direction was horizontal and perpendicular to the flow, the $y$ direction was parallel to the flow, and the $z$ direction was vertical. These measurements were corrected for the center tube bias error discussed in the Modeling Sensor Errors section, Fig. 3-10. A separate plot of the measured biases of the 15centimeter path vorticity meter with its axes labeled is shown in Appendix C. Both sensors have the same ratio of center stalk diameter to path length; the buoy center stalk had to be this large to be strong enough to be picked up. The pod diameter to path length 


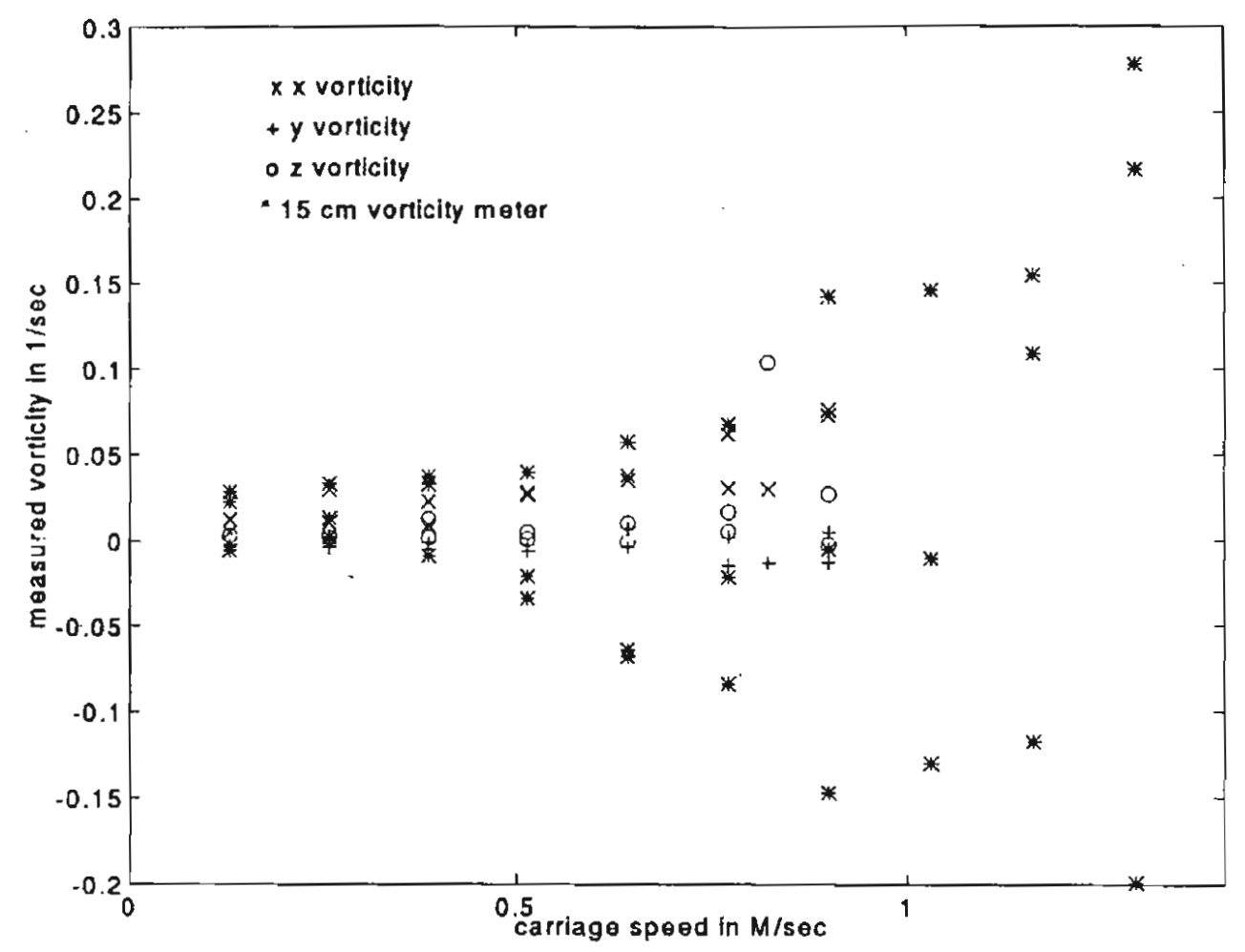

Fig. 3-9 Uncorrected vorticity bias of 15- and 45-centimeter path sensor in constant flow.

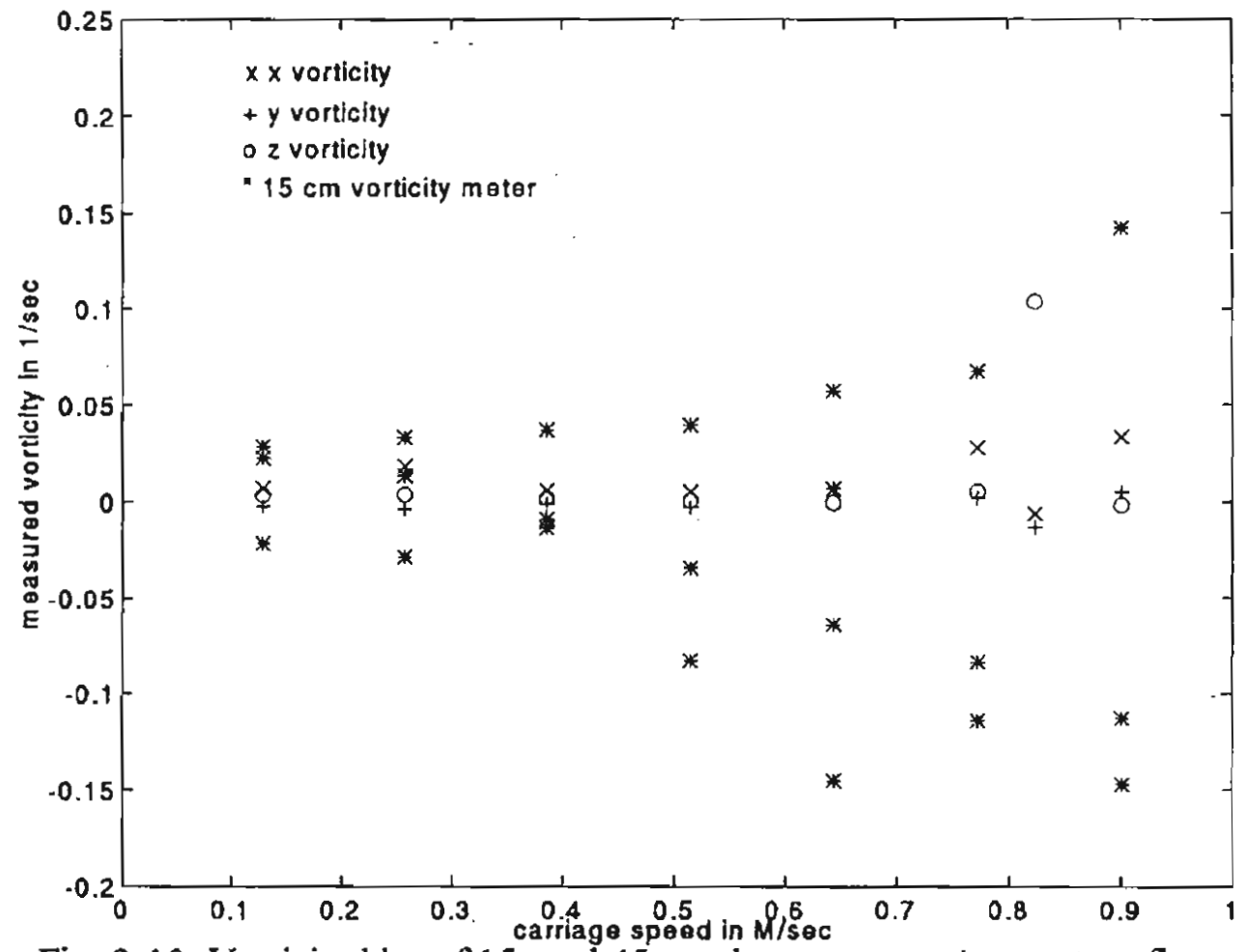

Fig. 3-10. Vorticity bias of 15- and 45-centimeter sensor in constant flow using stalk-bias correction described in text. 
is $62.5 \%$ smaller for the larger $45 \mathrm{~cm}$ path sensor. Correcting for center tube bias reduced bias to below $0.01 / \mathrm{s}$ in constant flow up to $70 \mathrm{~cm} / \mathrm{s}$. Above $70 \mathrm{~cm} / \mathrm{s}$, the pod arms strummed in the parallel, double frequency mode and measurements of velocity noise drastically increased. The $x$-axis (cross channel) bias error at $0.257 \mathrm{~m} / \mathrm{s}$ corresponds to the wave speed of a wavelength just longer than the diameter of the center tube. The depth-varying, extra drag at this speed is a Froude-number, free-surface effect of the test, resulting from the center stalk penetrating the water surface. In an actual deployment, all of the center stalk is underwater and this Froude effect should not exist at that velocity.

Correcting for the center stalk bias leaves mostly pod wake errors which scale as the pod diameter over the path length. The biases corrected for the center stalk wake for both size sensors for a $0.643 \mathrm{~m} / \mathrm{s}$ tow are listed in Table 3-1. The ratio of these biases is about nine. The three-dimensional pod-wake model predicted that vorticity bias would scale as $\frac{U}{L}\left(\frac{d}{L}\right)^{\frac{3}{3}}$. Using the dimensions of the 15-centimeter path and 45-centimeter path vorticity meters in the pod-wake model, the model predicts a ratio of bias of nine

\begin{tabular}{llcl} 
& \multicolumn{3}{c}{ Corrected bias } \\
& $\mathrm{x}$ & $\mathrm{y}$ & $\mathrm{z}$ \\
15-cm path sensor & -0.068 & 0.057 & -0.064 \\
45-cm path sensor & 0.0067 & 0.007 & -0.006 \\
ratio & 10.2 & 8.2 & 10.7
\end{tabular}

Table 3-1. Vorticity sensor bias in $0.643 \mathrm{~m} / \mathrm{s}$ constant flow corrected for center stalk bias.

Comparing error data from the tow tank and strumming onset speed, shows one of the design tradeoffs. The 45-centimeter path sensor was designed with small cross sections to reduce wake effects which reduced error, but this also lowered the strumming onset speed. For any sensor size, there is a tradeoff between the error within an operating envelope and the size of that operating envelope. 


\section{VARIANCE IN CONSTANT FLOW}

The size of velocity fluctuations caused by vortex shedding was measured. The geometry is shown in Fig. 3-11 where $\mathrm{D}$ is the center stalk diameter and $\mathrm{L}$ is the acoustic path length. The expected fluctuating velocity is given by equation (3-18).

$$
\Delta \text { velocity }=\frac{\int u \cdot d l}{L}=\text { constant } * \frac{D U_{\infty}}{L}
$$

The velocity of the vortices shed scale with the free-stream velocity and the vortice's size should scale with the stalk diameter. Acoustic measurement of velocity line averages the fluctuating velocity over the acoustic path. The constant in this parameterization was measured by towing the 15-centimeter path sensor through still water Fig. 3-12. The measured constant varied from 1.2 to 1.8 .

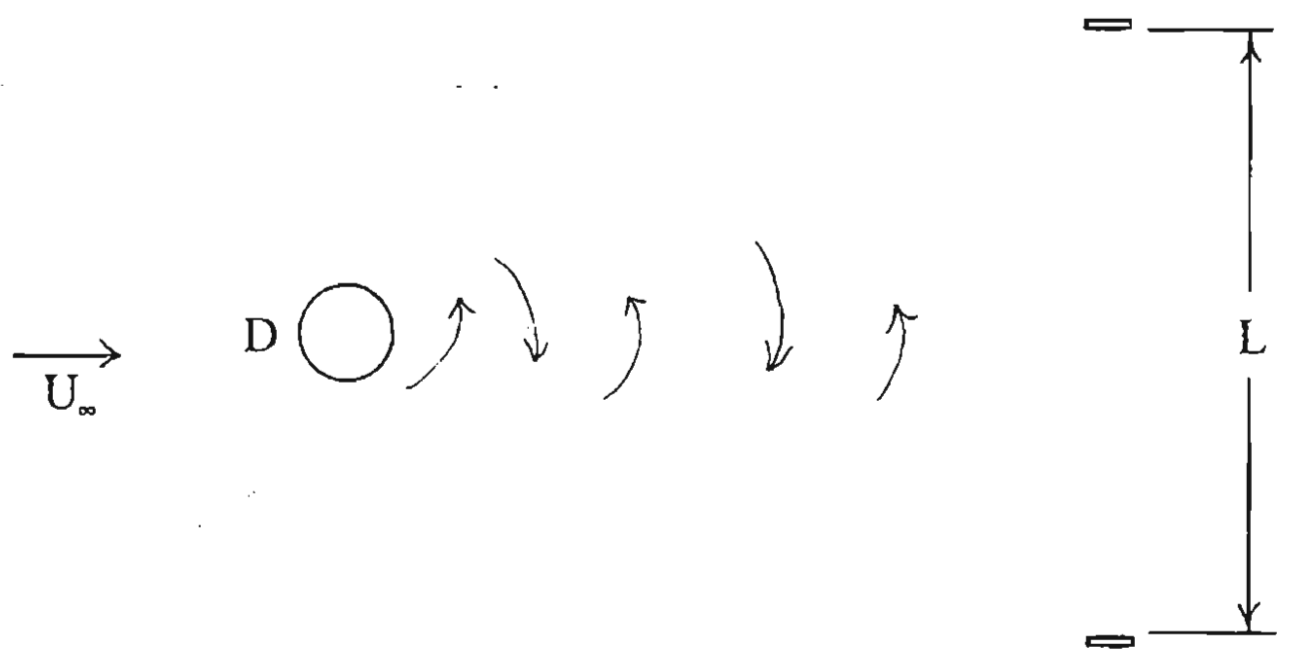

Fig. 3-11. Geometry used to measure size of shed-vortex, measured velocity variance. 


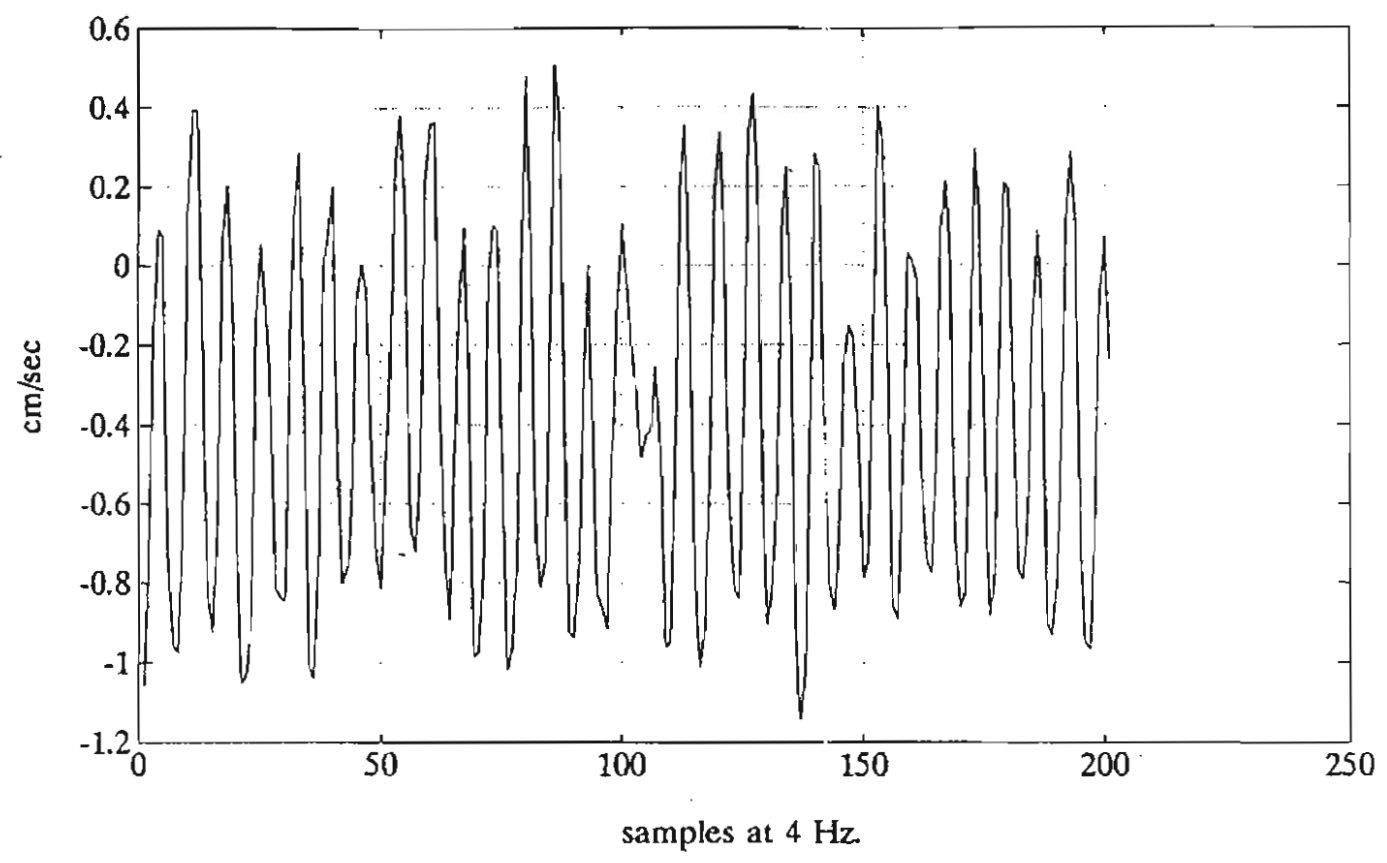

Fig. 3-12. Fluctuating measured velocity perpendicular to and behind center stalk of $15 \mathrm{~cm}$ path vorticity meter in $4.06 \mathrm{~cm} / \mathrm{s}$. tow.

Fluctuations of velocity perpendicular to the center stalk and tow velocity, were measured to increase with tow velocity. A power spectral density of this measured velocity at one tow speed is shown in Fig. 3-13. The large spectral peak in the center of this figure corresponds to the shedding frequency of the center stalk. Spectra of cross velocity, such as shown in Fig. 3-13, were measured at different tow speeds and are compiled together in Fig. 3-14. As the carriage moved faster, the peak frequency increased and velocity variation amplitude increased. The peak at the highest speed was aliased and was actually above the Nyquist frequency. At the carriage speeds run, vortex shedding variance from the pod arms was aliased. 


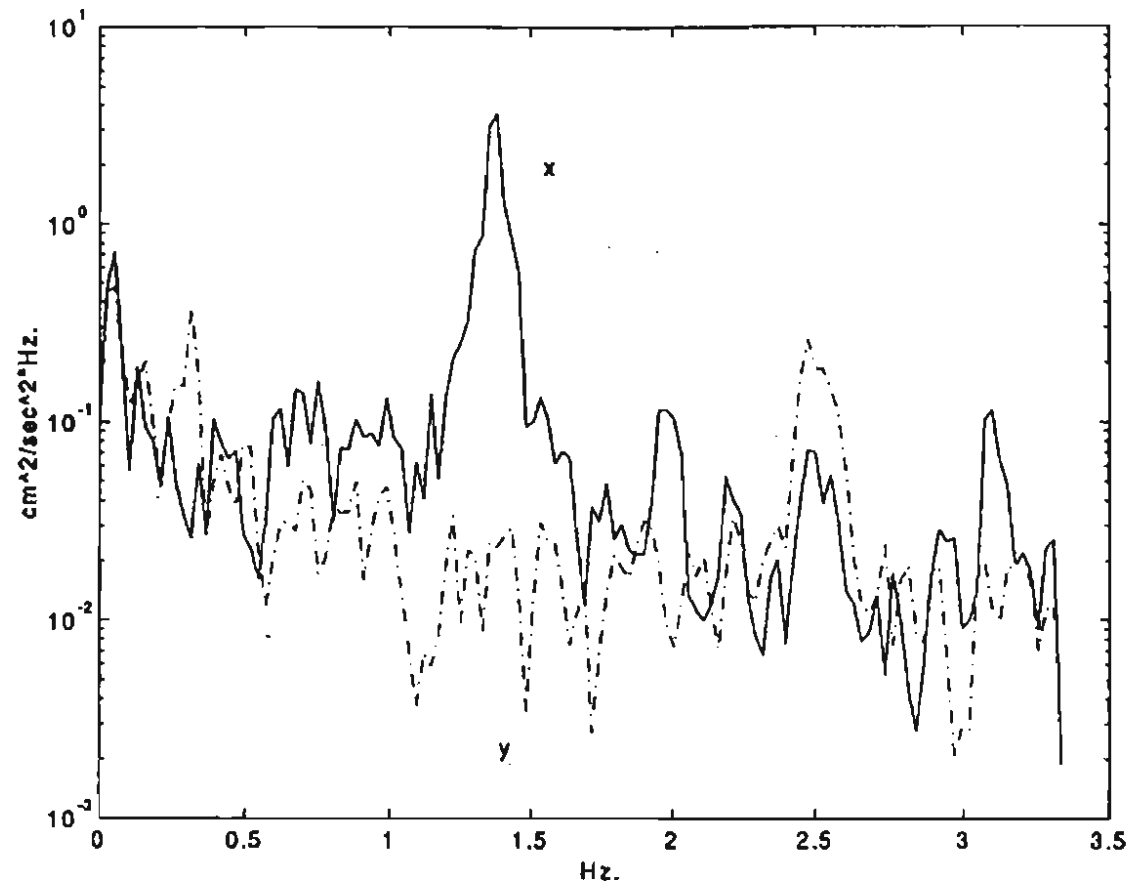

Fig. 3-13. Power spectral density of measured cross tank $X$ and parallel $Y$ velocities of a $45-\mathrm{cm}$ path sensor towed at $0.26 \mathrm{~m} / \mathrm{s}$ showing the Strouhal peak in cross velocity.

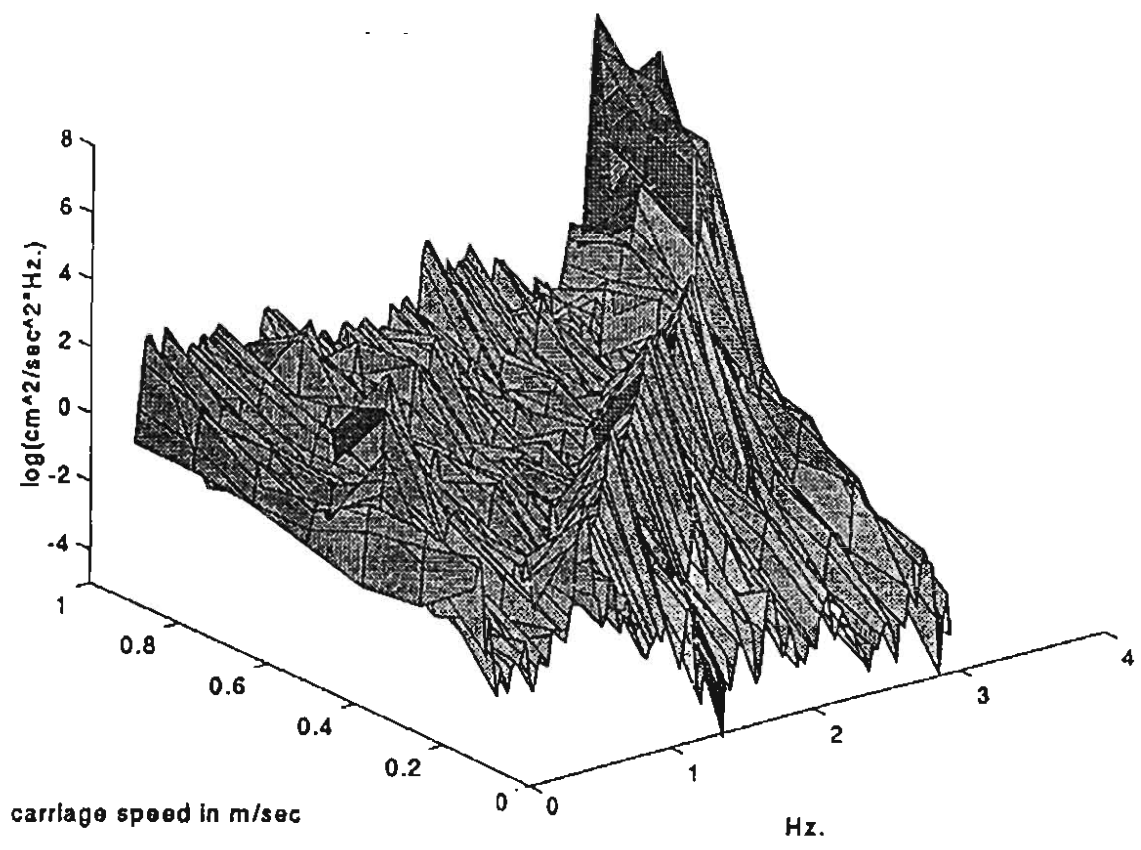

Fig. 3-14. Power spectral density of measured cross tank velocity at different carriage speeds. 


\section{BIAS IN WAVE FLOW}

Measurement bias of mean vorticity in a wave flow is difficult to accurately measure or model. Vorticity errors in a wave flow are primarily from the sensor's wake advecting back into the measurement volume. Subtle differences in how and where the wake is advected back could significantly affect errors. Constant flow models where the sensor wake is only advected away from the measurement volume cannot be directly applied to a wave flow.

A nondimensionalization of error in a monochromatic wave field is equation (319). In this equation, $\omega_{\text {bias }}$ is the vorticity bias, $L$ is the path length, $u$ ' is the rms relative

$$
\frac{\omega_{\text {bias }} L}{u^{\prime}}=F\left(\text { geometry, } \frac{T u^{\prime}}{L}, \frac{g L}{u^{\prime 2}}, \frac{h}{L}, \theta, \frac{z}{L}, \frac{\bar{u}}{u^{\prime}}, \frac{\bar{\nu}}{u^{\prime}}\right)
$$

velocity, $T$ is the wave period, $g$ is the acceleration due to gravity, $h$ is the water depth, $\theta$ is the heading angle of the sensor with respect to the waves, $z$ is the sensor depth, $\bar{u}$ is the average flow past the sensor parallel to the waves, and $\bar{v}$ is the average flow past the sensor perpendicular to the waves. The $T u^{\prime} / L$ term is the square root of two multiplied by the Keulegan-Carpenter number which is proportional to the wave excursion divided by the sensor diameter (Faltinsen, 1990). The $g L / u^{\prime 2}$ term is an inverse Froude number for the relative wave velocity. This nondimensionalization is convenient to apply to laboratory rotating arm tests of bias, wave tank tests of bias, and is also convenient for estimating the accuracy of a measurement after it has been made. This formulation is not, however, the most convenient to apply in a predictive role given an expected sea-state without knowing the buoy response function. The measured buoy response functions are plotted in Appendix C.

In the tests performed so far $g L / u^{\prime 2}, \theta$, and mean drift $\bar{u}$ were varied. To keep geometry constant, only data taken with the 45 -centimeter path sensor is discussed here. Because of the sensor size, the water depth and sensor depth could not be significantly 
varied in laboratory tests. Cross wave velocity drift, $\bar{v}$, was zero for all the data taken so its effect cannot be estimated from the data. The parameters that I varied were wave period, wave amplitude, sensor angle with respect to waves, and average flow past the sensor parallel to the waves. This section discusses data taken with the sensor on a rotating arm in still water to measure bias and estimate how bias varies with relative rms velocity and down-wave drift. Data taken in a wave tank and during an open-ocean deployment are used to show that sensor heading does not significantly affect sensor bias. I use data from a buoy deployment south of Martha's Vineyard, MA. with no wind stress but significant swell, to estimate bias magnitude in waves.

\section{ROTATION ARM BIAS}

This section measures the vertical and down-wave vorticity biases to be less than 1 $\times 10^{-2} / \mathrm{s}$, measures bias sensitivity to down-wave drift, and shows that cross-wave bias is proportional to rms relative wave velocity. The 45 -centimeter path sensor was mounted on a rotating arm at the David Taylor Model Basin. The rotating arm traversed the sensor such that all points of the sensor traveled in the same, but displaced, circles. Any line in the sensor stayed in the same direction as the arm traversed.

To measure vorticity sensor bias in wave-like flow, the sensor was traversed in circles relative to the carriage at different rates while the tow carriage traveled at different speeds. A typical plot of measured vorticity means for the same arm rotation rate, at different carriage speeds is shown in Fig. 3-15. In this plot, the arm was rotated at a period of seven seconds while the carriage speed was stepped through different speeds. Each point is a measured mean of at least two minutes of data. The $\mathrm{x}$-direction vorticity is across the tank, parallel to the axis of rotation of the rotating arm, and is corrected for the center stalk bias described in the Modeling Sensor Errors section. The y-direction vorticity is down the tank parallel to tow carriage velocity and the z-direction is vertical. Included on the plot are lines of standard error. The standard error of a mean is the standard deviation of a signal divided by the square root of the number of independent samples of the signal that were used to calculate the mean. The standard error is a 


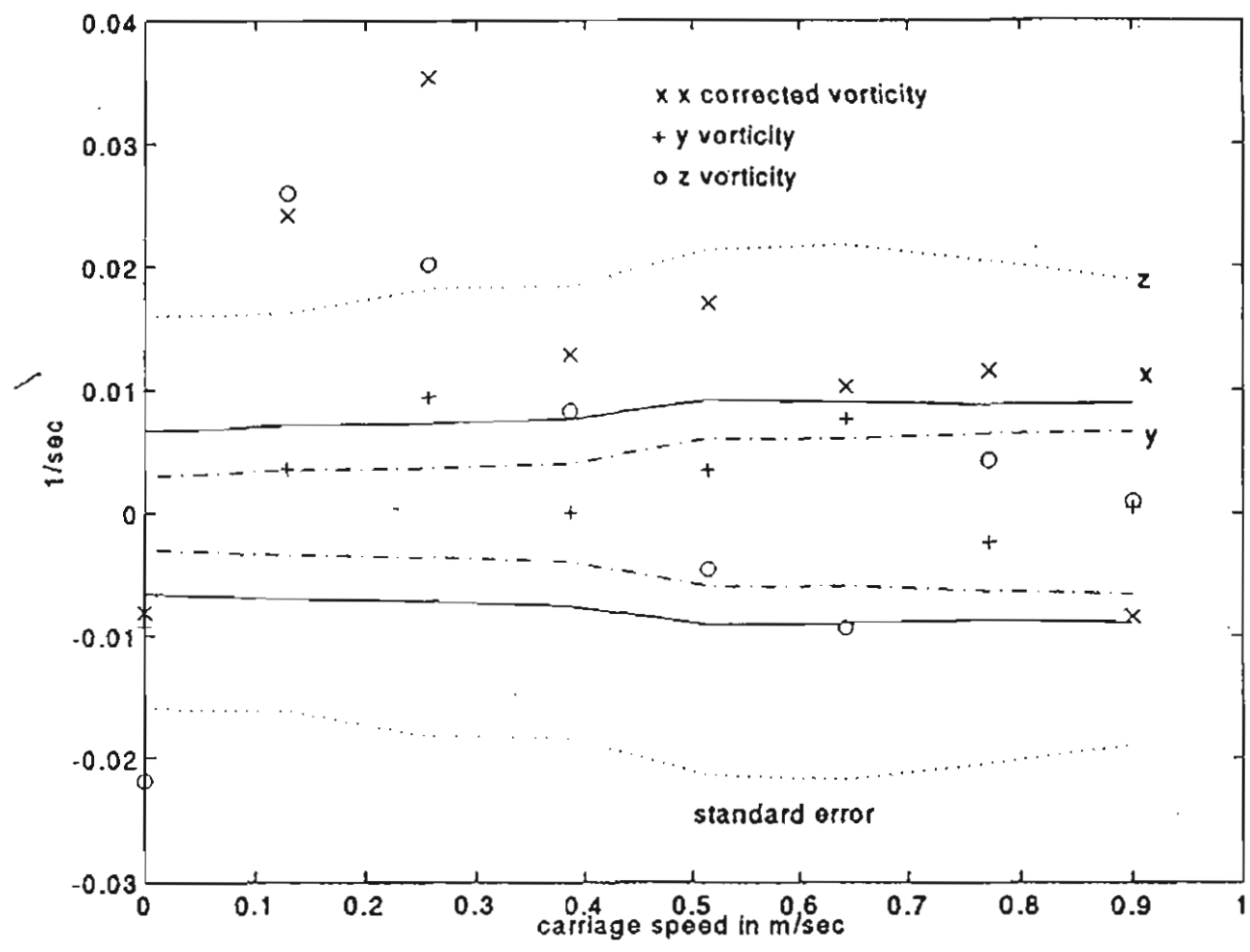

Fig. 3-15. Vorticity means of the 45-cm path vorticity meter measured on the rotating arm with a seven second period. The standard errors of each axis are also plotted.

measurement of the variability of a sample mean. The standard errors in these measurements were not small because the vorticity variance was large. The Strouhal wakes behind bluff bodies contain a great deal of time varying vorticity; the standard deviations of vertical vorticity for the data taken shown in Fig 3-15 were about 0.5 per second. Plots of vorticity means for different arm rotation rates are shown in Appendix C.

To measure whether the down-wave vorticity and vertical vorticity were biased, the measured means are plotted divided by their standard errors and compared to a normal distribution. The down-wave, y axis, vorticity means divided by their standard errors for all the rotation rates and carriage speeds are plotted in Fig. 3-16, and the vertical, $z$ axis, results in Fig. 3-17. In both these figures, the number of independent samples was 


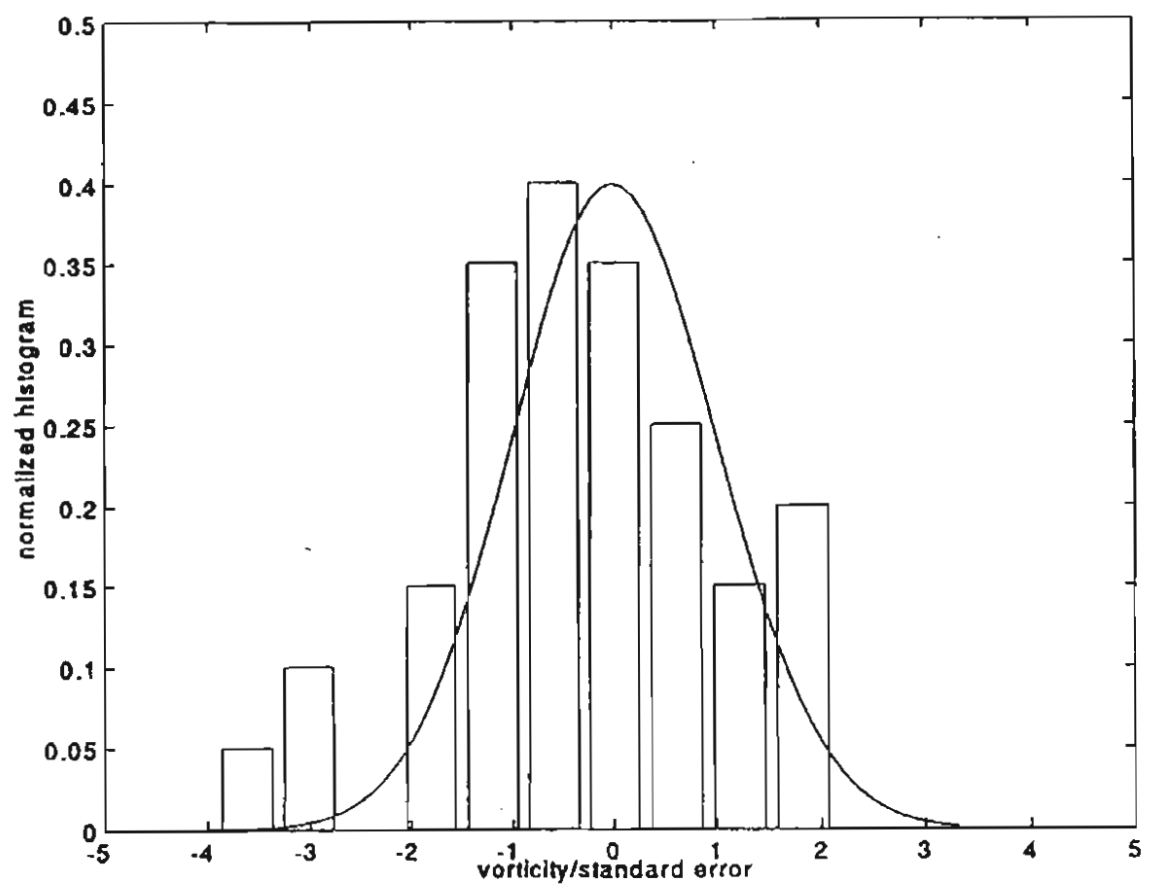

Fig. 3-16. Down-wave vorticity means divided by their standard errors compared to a normal distribution.

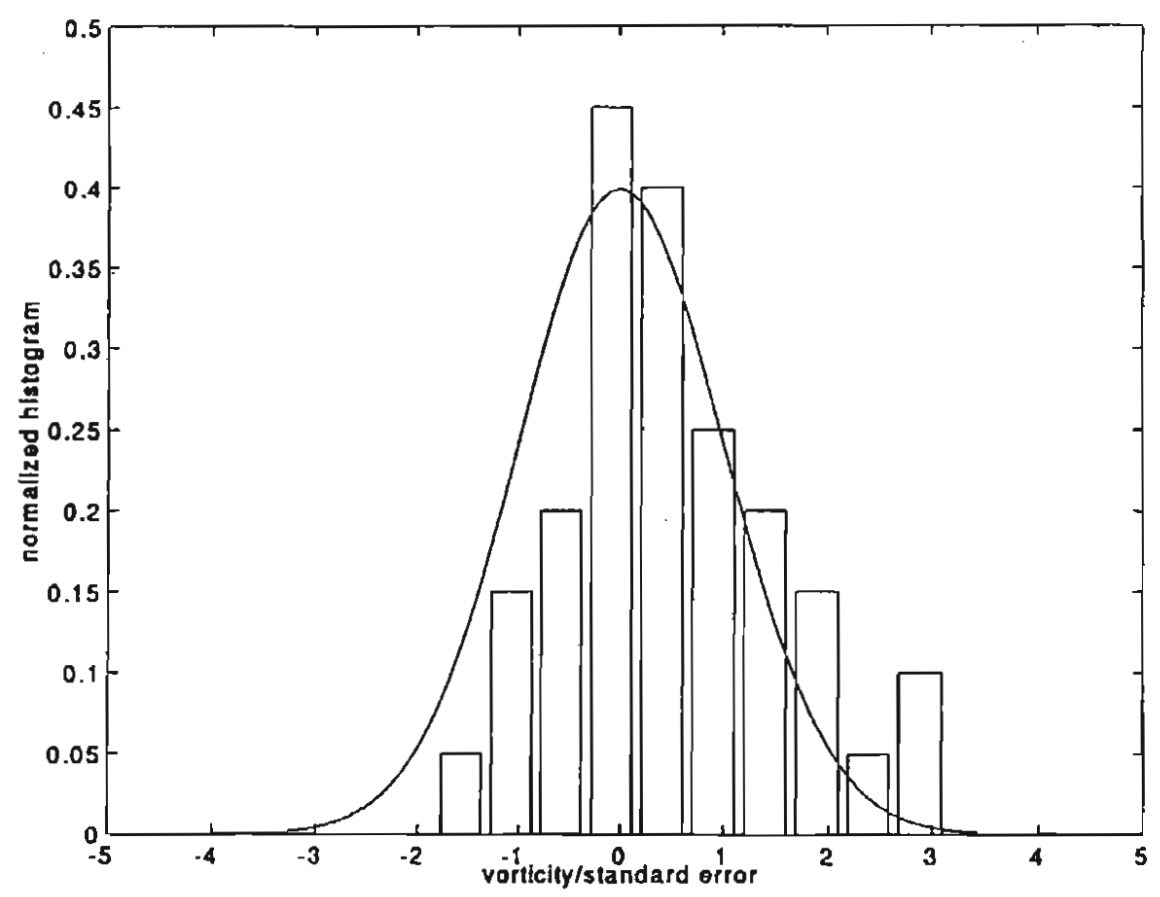

Fig. 3-17. Vertical vorticity means divided by their standard errors compared to a normal distribution. 
assumed to be half the number of samples of the means. An autocorrelation of a vorticity measurement made during a rotating arm test is shown in Fig. 3-18. The width of the autocorrelation function at half its peak value is two samples. Figures 3-16 and 17 show that there are no statistically significant biases of down-wave or vertical vorticity. If there are biases in either of these measurements, the biases are less than $1 \times 10^{-2} / \mathrm{s}$.

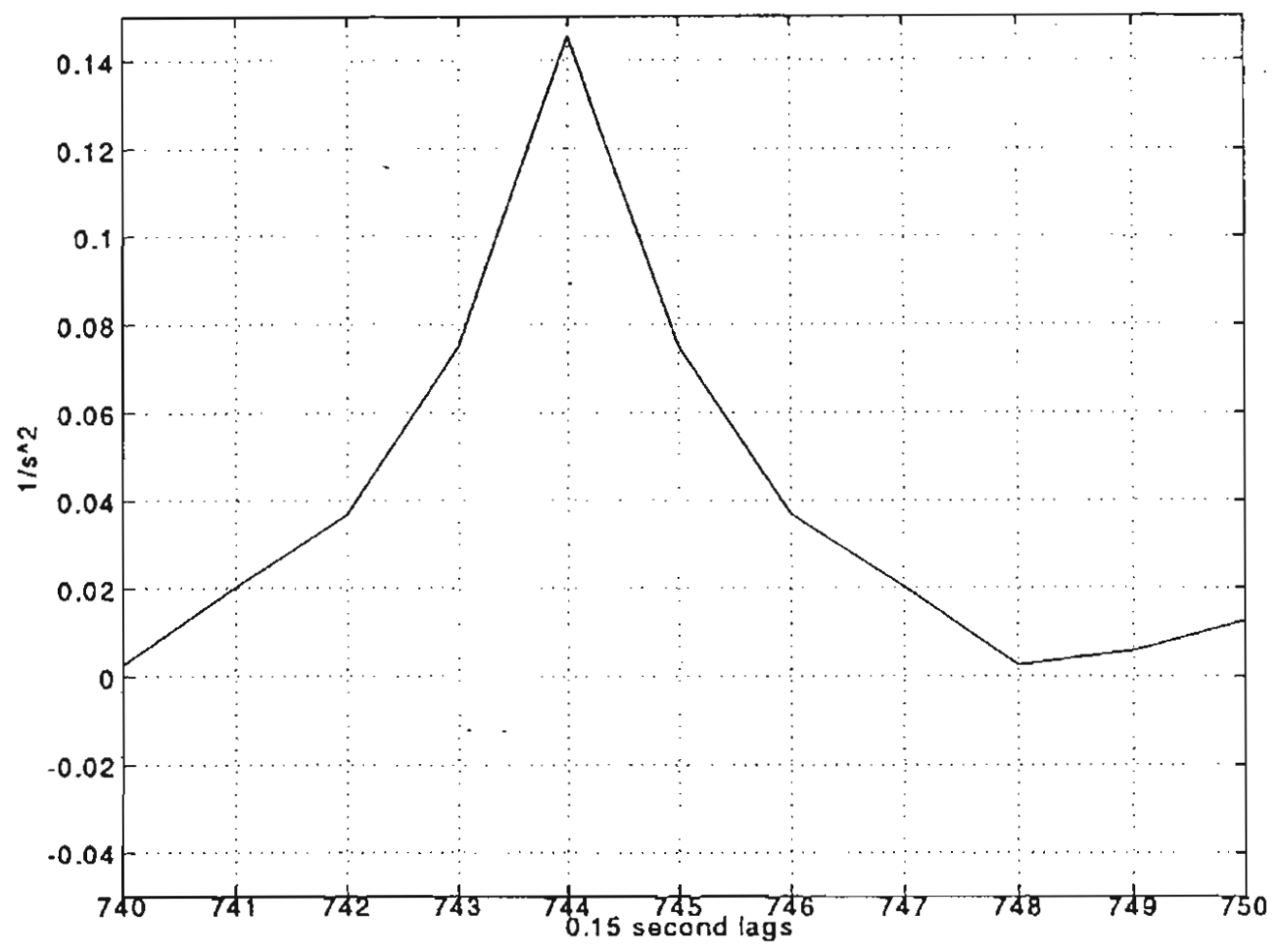

Fig. 3-18. Autocorrelation of vorticity measurement during a rotating arm test showing an autocorrelation time scale of two samples.

To measure the influence of the down-wave drift velocity, $\bar{u}$, on bias, I performed rotation-arm tests at different tow carriage speeds and rotation rates. The data from these tests are plotted in Fig. 3-19. The abscissa is the relative drift velocity divided by the relative wave velocity, and the ordinate is the mean vorticity scaled by the acoustic path length and relative velocity. This data shows that relative, down-wave drift velocity $\bar{u}$ does not strongly effect bias.

Vorticity bias was found to scale with relative, rms velocity. Bias for rotation arm 
tests with the same ratio of drift speed to circular wave velocities are plotted as a function of rms relative velocity in Fig. 3-20. That errors scale with relative velocities in a wave flow, makes sense because wake velocities scale with relative flow and these wakes advected back into the measurement volume cause the bias error.

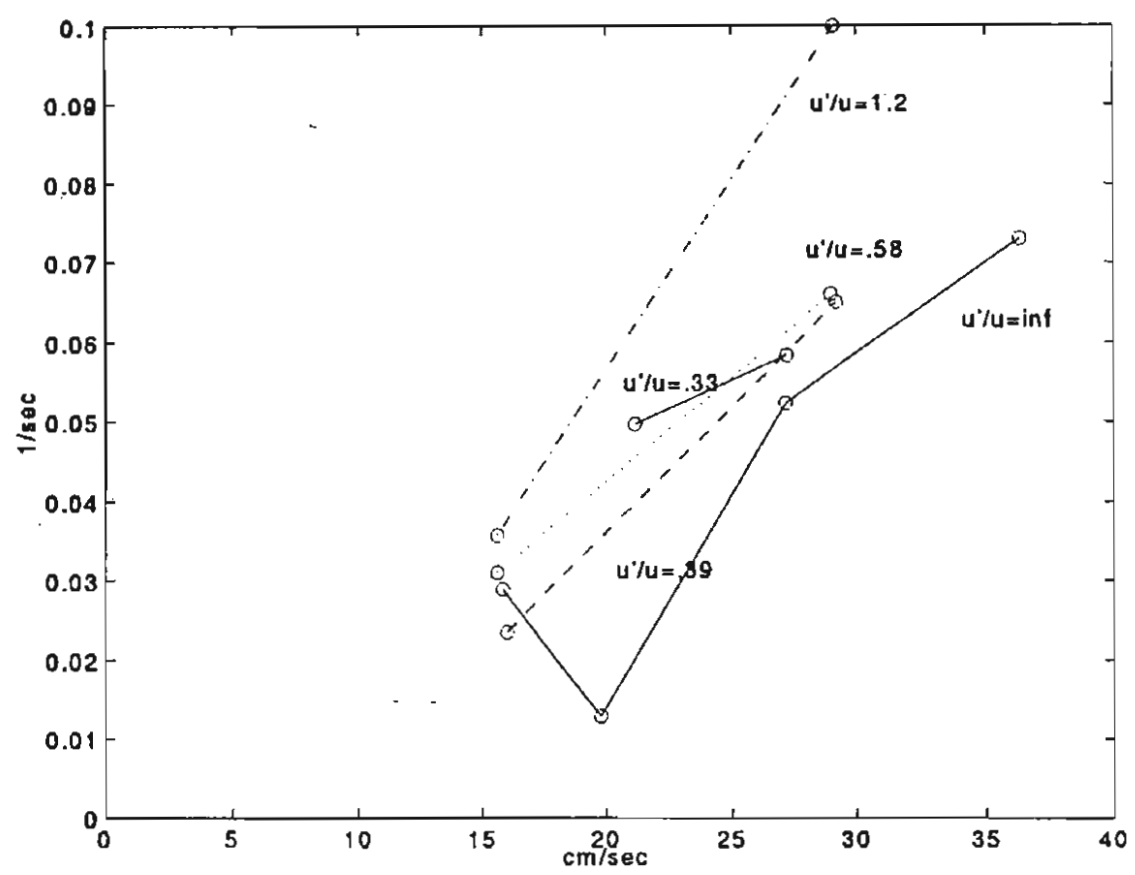

Fig. 3-19. Rotating-arm, sensor vorticity means plotted with respect to relative drift velocity.

Data from these tests have to be used carefully to infer wave bias conclusions, because a rotating arm test is a model of wave flow, and the rotating arm vibrated strongly. A rotating arm test is a model of wave flow because while the flow moves relative to the sensor in the circular paths like a wave flow, the pressure gradients on the sensor are not the same as in a wave field. Pressure gradients that are parallel to flow, affect boundary-layer separation and therefore affect wakes left by the sensor. Sensor wakes advecting back into the measurement volume are the dominant bias errors, 


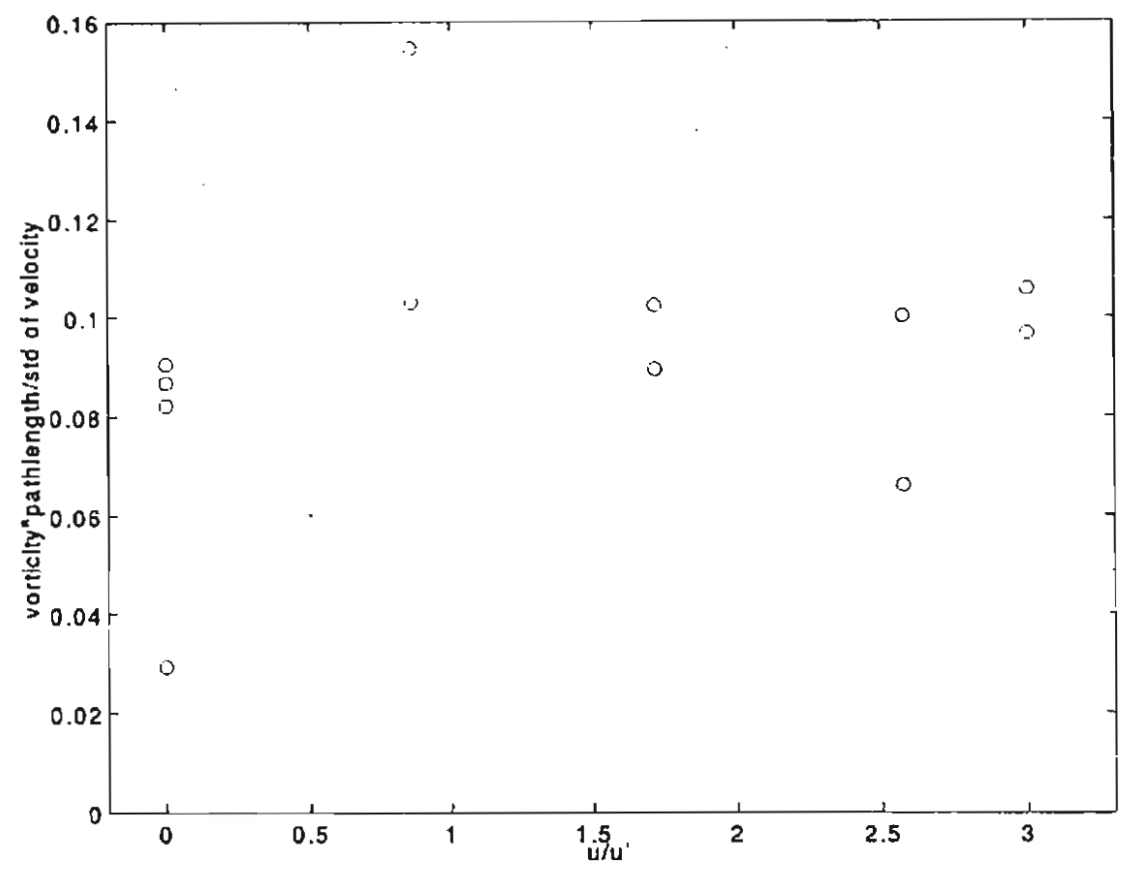

Fig. 3-20. Rotating-arm vorticity means for constant drift velocity divided by wave velocity plotted with respect to relative rms velocity.

therefore anything that affects boundary layer separation on the sensor could influence the sensor bias.

The rotation arm used vibrated strongly. The power spectral density of measured horizontal acceleration is shown in Fig. 3-21; it indicates aliasing of vibration and motion. These spectra can be compared to the acceleration spectra from an ocean deployment Fig. 3-22. The buoy's most effective anti-alias filter is the second-order, lightly-damped oscillator that is the buoy response. The fastest natural frequency of the buoy is in heave with a period of about a second; pitch and roll frequencies are slower. In the ocean acceleration spectra, the spectral magnitude above three hertz is the measured noise level of the accelerometers. Sensor vibration can also affect boundary-layer separation and wake formation. The buoy, when deployed in the ocean, does not have aliasing problems, but when strapped rigidly to the vibrating rotating arm did have aliasing. 


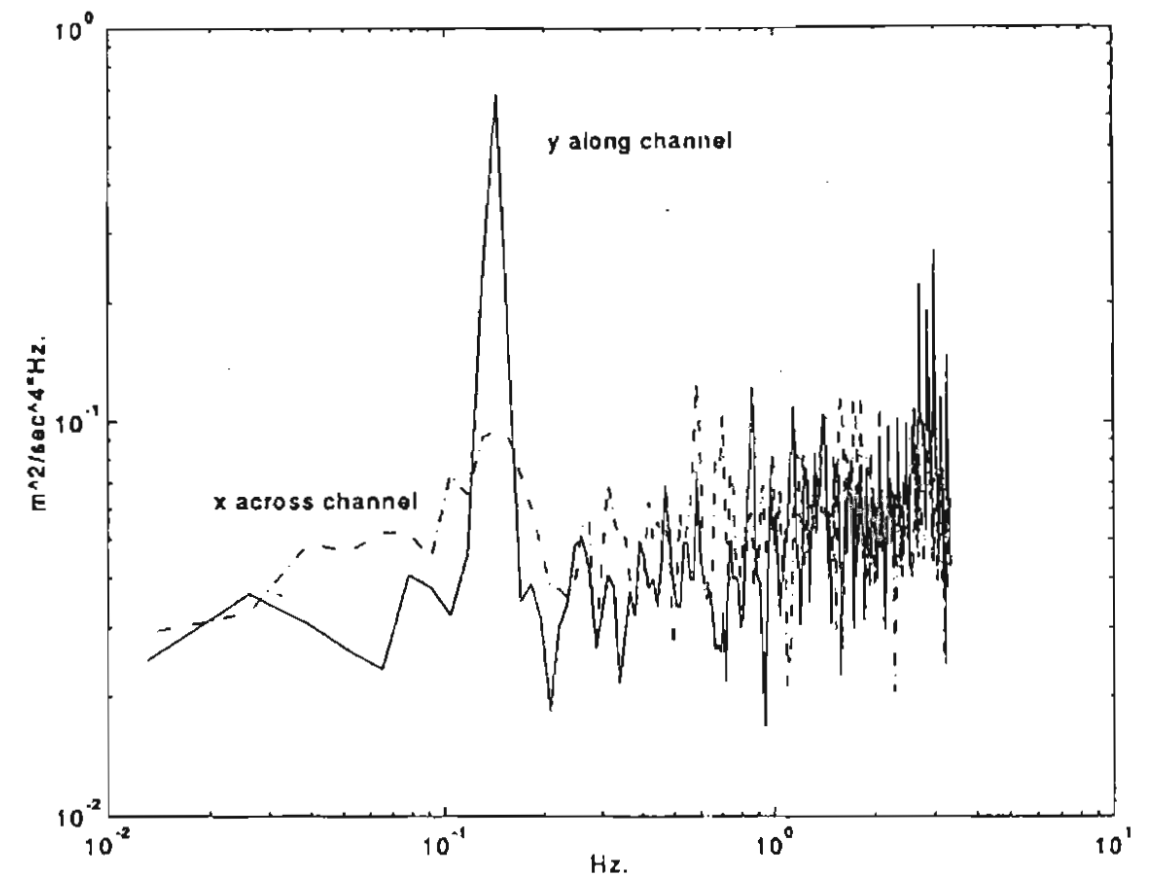

Fig. 3-21. Horizontal acceleration spectra from rotating arm test showing strong vibration.

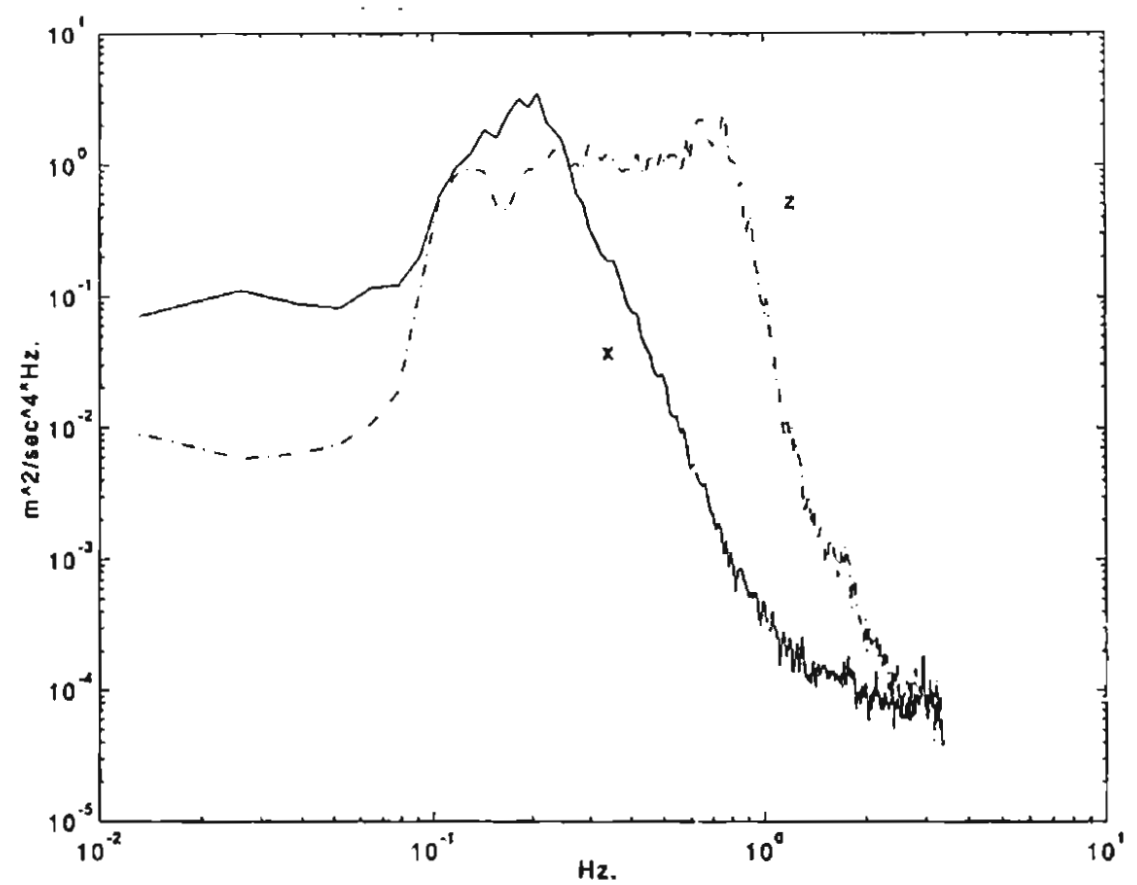

Fig. 3-22. Open-ocean deployment acceleration spectra; $\mathrm{X}$ is horizontal acceleration, and $\mathrm{Z}$ is vertical acceleration. 


\section{WAVE BIAS SENSITIVITY TO ORIENTATION}

Sensor bias was measured to not vary significantly with sensor orientation in a wave tank. Wave tanks have real wave flow which avoids the unmodeled pressure gradients of a rotating arm test. The actual shear drifts can, however, be non-zero. In a wave tank, the sensor is held still while waves radiate past. The buoy in the ocean, however, moves with the longer wavelength swell and wave flow relative to the buoy is more complicated. Again, I inferred the sensitivity of an error parameter from a test that does not precisely reproduce flow parameters.

The wave tank used was in the Canada Center for Inland Waters and when used carefully, was stationary and measurements were repeatable. Tank flow measurements had to be averaged for at least ten minutes to get consistent measurements. Fig. 3-23

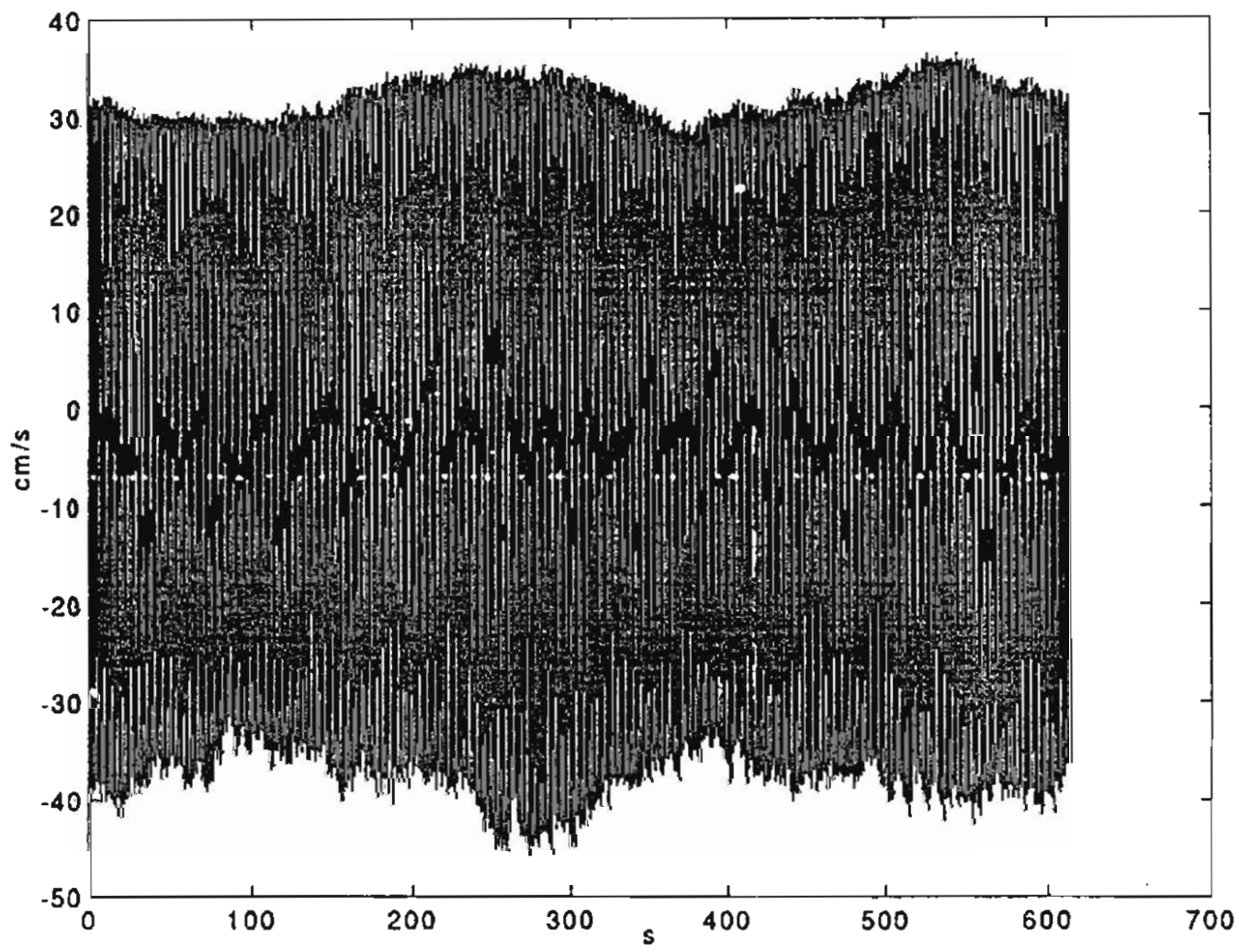

Fig. 3-23. CCIW wave tank velocity measurements made with an acoustic current meter showing low frequency variability. The velocity trace in this long time series of a rapidly varying signal, is smeared and aliased by the printing but does show the time varying envelope of tank velocity. The sampling at $10 \mathrm{~Hz}$. of a wave of a several second period is not aliased. 
shows a velocity measurement that had significant low frequency variability. Ten-minute averages provided consistent mean flow measurements to about $1 \mathrm{~mm} / \mathrm{s}$, even when the elapsed time between measurements was over three hours.

The wave tank testing took advantage of the tank's stationarity. The mean flow pump and wave maker were started and run at least two hours for tank transients to die down before any measurements were made. With the vorticity sensor out of the water, ten-minute, mean current measurements were made at the top and bottom depths of the vorticity measurement volume. BASS and Denshi Kogyo (a Japanese instrument maker) acoustic current meters were used. The current meters were removed from the water, the vorticity sensor was put in the water, and at least a ten-minute measurement of vorticity was made without wake disturbance from the current meters. The vorticity meter was removed from the water, the current meters put back in, and ten-minute current measurements were made again at the top and bottom of the measurement volume to see if the wave tank drift currents had changed over the elapsed time. The wave tank was found by all the instruments to be stationary but had large shears, of order $0.11 / \mathrm{s}$. This shear is larger than typical for unstratified geophysical flows. Current meters have unknown errors in wave flows and the BASS and Denshi Kogyo current meter measurements of shear often differed by more than $0.04 / \mathrm{s}$. The wave tank was too wide to use a laser Doppler velocity meter.

The consistency of current meter measurements did however show tank stationarity that was used to measure how vorticity meter bias changes with orientation. Under one set of wave and current conditions, the vorticity sensor was measured at different heading angles relative to the waves. The mean vorticity measurements were rotated into tank coordinates and are listed in Table 3-2. Because of sensor symmetry about the vertical axis, the three physical angles are repeated every 120 degrees and mirrored every 60 degrees. Sampling at these three angles is equivalent to sampling every 15 degrees about the vertical axis. No changes in measured horizontal vorticity greater than $0.014 / \mathrm{s}$ were caused by sensor angle. The measured vertical vorticity, however, 
changed by $0.02 / \mathrm{s}$. A second measurement of wave bias sensitivity to orientation, made in the open ocean, showed less change with orientation, and will be described in the next section.

\begin{tabular}{clll} 
& \multicolumn{2}{c}{ Mean Vorticity } & \\
Sensor Orientation & Parallel to Tank & Cross Tank & Vertical \\
$0^{\circ}$ & -0.0093 & 0.1159 & 0.0251 \\
$-15^{\circ}$ & -0.0102 & 0.1055 & 0.0101 \\
$-30^{\circ}$ & -0.006 & 0.1064 & 0.0048
\end{tabular}

Table 3-2. Measured vorticity means in tank coordinates at three sensor rotation angles.

\section{OPEN-OCEAN BIASMEASUREMENT}

The buoy was deployed in the open ocean with negligible wind stress and significant swell to measure instrument bias in waves. The deployment was south of Martha's Vineyard MA. after a storm. An actual deployment "simulates" all error terms; the only shortcoming is that the true shear is not accurately known. Mean vorticity measured by the shallow sensor along with the standard errors of each segment are shown in Fig. 3-24. Recall that vorticity is the curl of the velocity field and is therefore perpendicular to the corresponding velocities. Mean vorticity and corresponding standard errors measured by the sensor 2.45 meter deep are plotted in Fig. 3-25. These measurements will be used with some assumptions to estimate what instrument bias could be.

The dominant wave direction will be used along with the results from the rotating arm tests to estimate what part of the measured vorticity is real and what part could be bias. The wave directional spectrum of velocity was computed using the maximum entropy method and is shown in Fig. 3-26 (Longuet-Higgins, Cartwright, and Smith, 1963, and Lygre and Krogstad, 1986). Most of the waves were radiating at a heading of 45 degrees. The measured mean vorticity components were rotated into down-wave and 


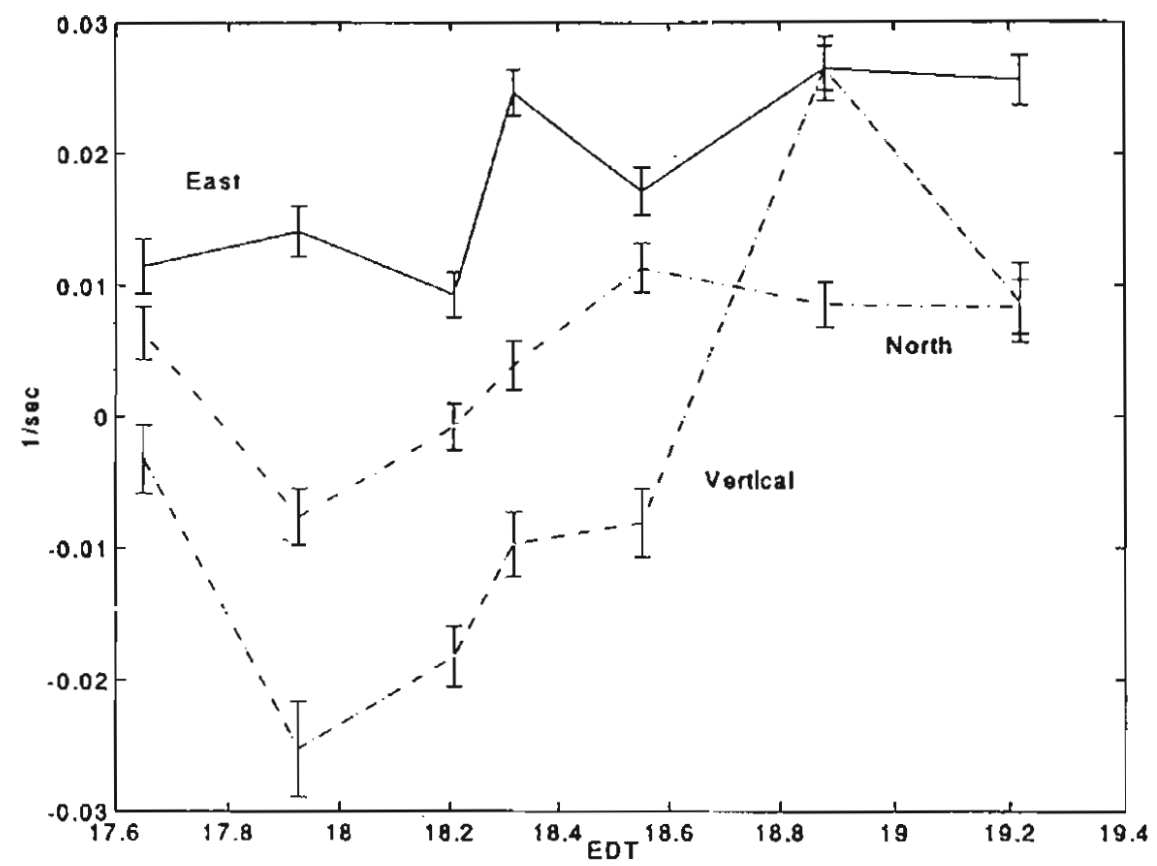

Fig. 3-24. Mean vorticity and standard errors measured by the sensor 0.83 meter deep during deployment south of Martha's Vineyard.

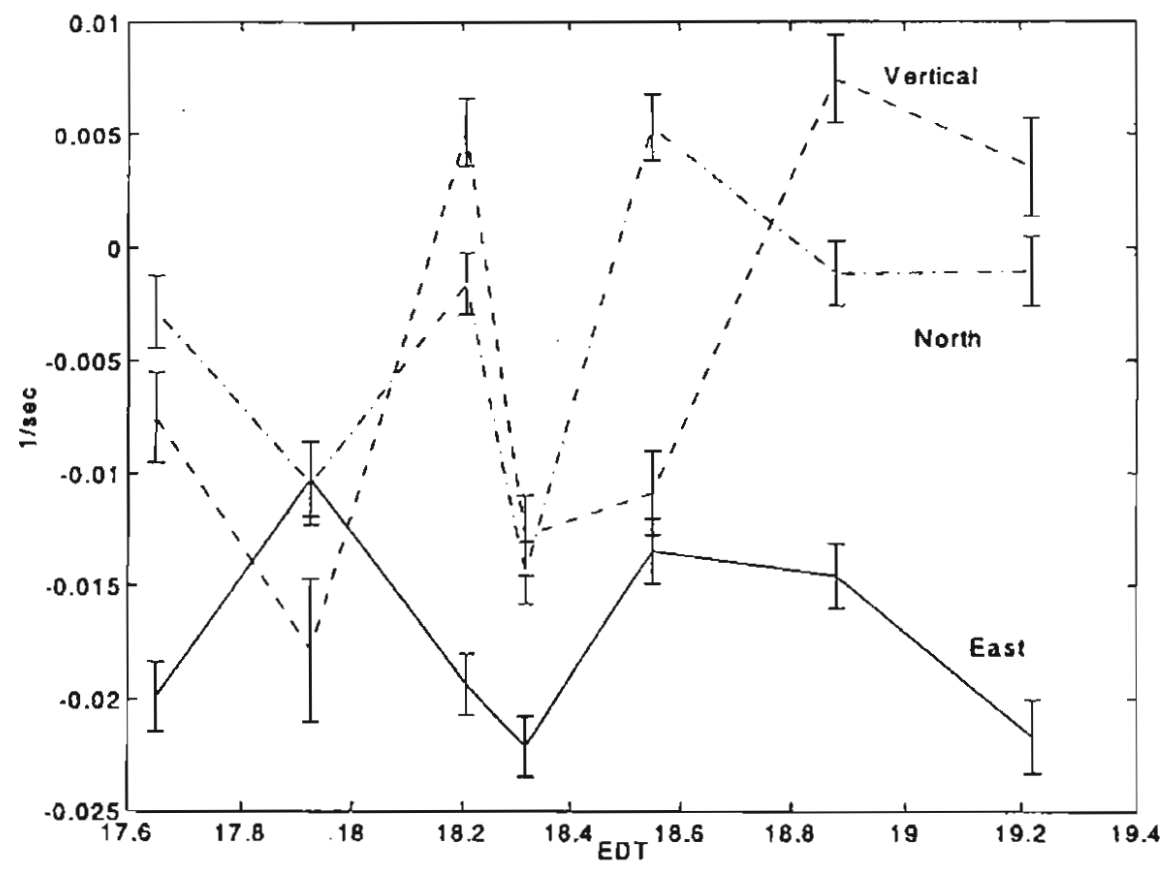

Fig. 3-25. Mean vorticity and corresponding standard errors measured 2.45 meter deep during deployment south of Martha's Vineyard. 


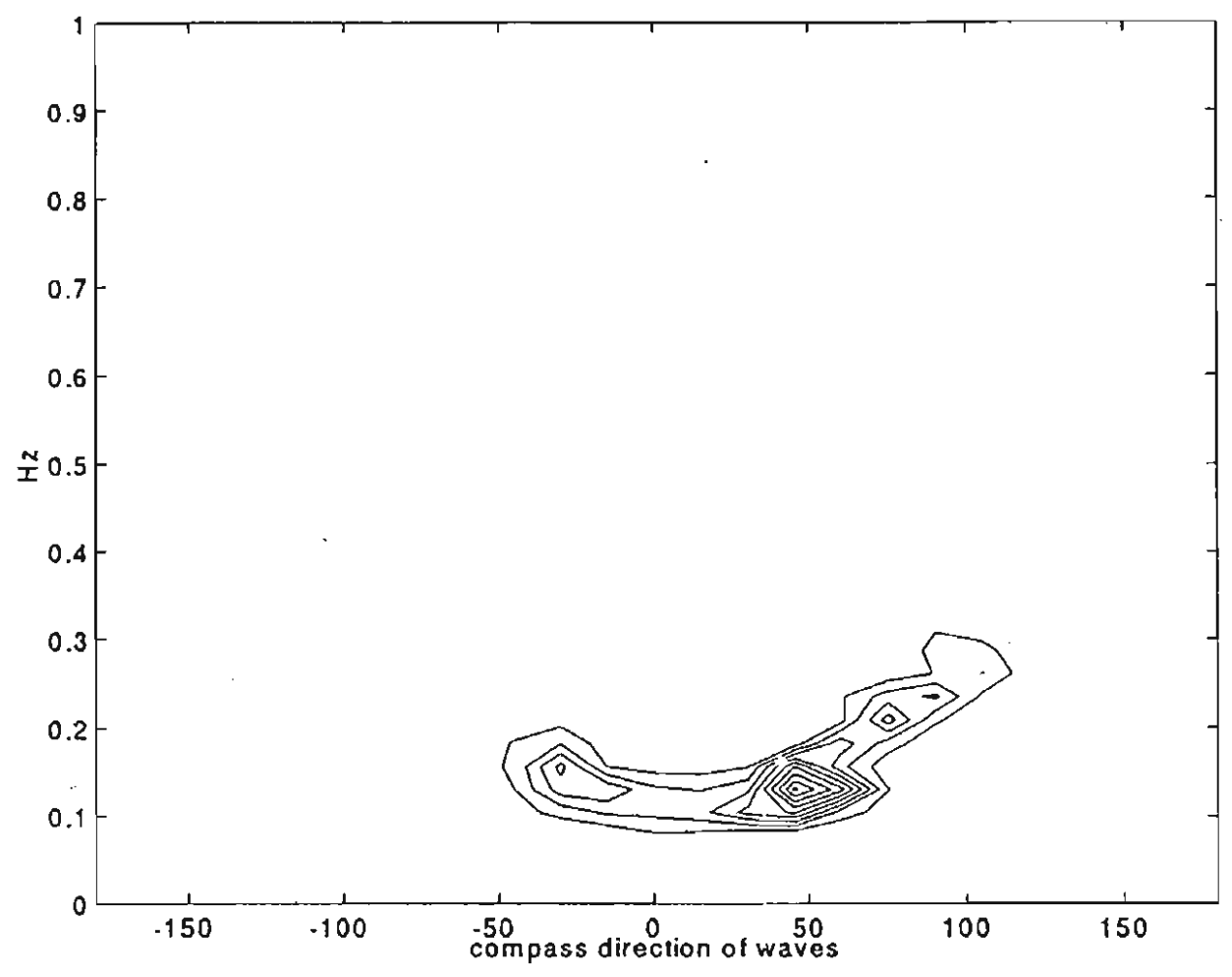

Fig. 3-26. Directional wave spectrum of velocity measured on the south of Martha's Vineyard deployment, calculated with the maximum entropy method.

cross-wave components with the coordinates defined by Fig. 3-27, and are listed along

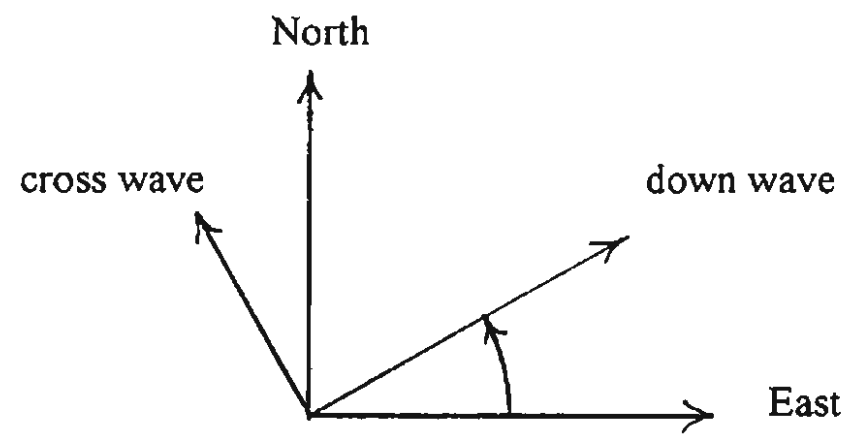

Fig. 3-27. Coordinate rotation from east, north and vertical to down-wave, cross-wave, and vertical.

with rms relative velocity in Table 3-3. The rotating arm tests showed no statistically 


$\begin{array}{cllll}\text { Sensor depth } & \text { down-wave } & \text { cross-wave } & \text { vertical } & \text { rms relative velocity } \\ 0.83 \mathrm{~m} & 0.016 / \mathrm{s} & -0.01 / \mathrm{s} & -0.0042 / \mathrm{s} & 28.1 \mathrm{~cm} / \mathrm{s} \\ 2.45 \mathrm{~m} & -0.015 / \mathrm{s} & 0.0096 / \mathrm{s} & -0.0047 / \mathrm{s} & 27.9 \mathrm{~cm} / \mathrm{s}\end{array}$

Table 3-3. Mean measured vorticity rotated into down-wave and cross-wave coordinates from deployment south of Martha's Vineyard.

significant bias in the down-wave or vertical directions. In this deployment, the downwave and vertical vorticities are assumed to be real and the cross-wave vorticities are assumed to be error. The rotating arm tests also showed that bias errors were proportional to rms relative velocity. The error bars presented in the applications section are these cross-wave vorticity means scaled by rms relative velocity.

The actual shear for this deployment could have been as large as $0.022 / \mathrm{s}$. Current shears in a stratified flow have very little vertical shear stress or mixing if the gradient Richardson number is above 0.25 . The measured Brunt-Vaisala frequency was 0.011 $\mathrm{rad} / \mathrm{s}$ giving a 9.4-minute buoyancy period. This stratification would have a Richardson number of 0.25 if the shear was $0.022 / \mathrm{s}$. If, on one hand, the actual shear was this large and in the direction measured by the buoy, the bias could have been zero. If, on the other hand, the actual shear was this large in the opposite direction of the buoy measured vorticities, the bias could have been larger.

If vorticity is assumed to be constant for a 20 minute period, the fact that the buoy rotated can be used to check the presumed zero bias in down-wave and vertical vorticity. Measured vertical vorticity is looked at first. A 20 minute time series of measurements was broken up into five degree heading bins and all measurements when the buoy heading was within a heading bin were grouped together. The mean measured vertical vorticity, standard deviation, and standard error were computed for each buoy heading bin and plotted in Fig. 3-28. The autocorrelation of vertical vorticity had a time scale of 5 samples; the number of samples used to compute standard error was divided by 5 to 


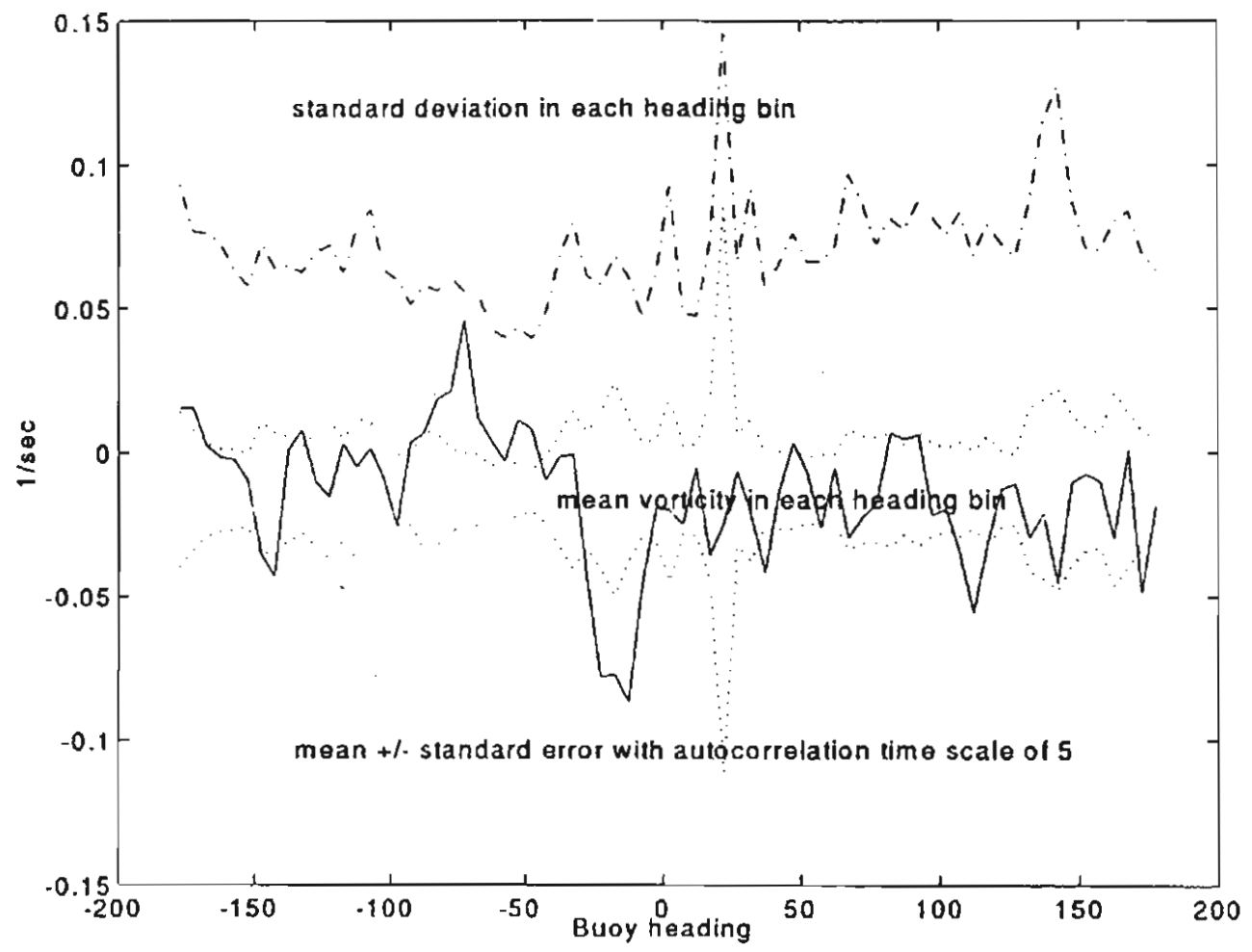

Fig. 3-28. Vertical vorticity broken up into buoy heading bins for a 20 minute record. Heading bin means, standard deviations, and standard errors are shown and indicate no significant bias that varies with heading.

estimate the number of independent samples. The sample means of each heading bin do not show any statistically significant bias that varies with buoy heading. The bias in vertical vorticity does not change with heading and the buoy and vorticity sensors have radial symmetry so the bias of vertical vorticity must be zero.

The presumed zero bias in down-wave vorticity was checked by breaking measurements of horizontal vorticity up into heading bins and assuming the actual vorticity was constant. If the actual vorticity is constant and the buoy rotates, the buoy referenced vorticity will be a sine wave when plotted with respect to heading. In Fig. 329. the heading bin means and standard deviations are plotted. Also plotted, as dotted lines, are the sine wave from the 20 minute-mean, earth-referenced vorticity rotated into buoy coordinates, with the standard error added and subtracted from this sine wave. The 


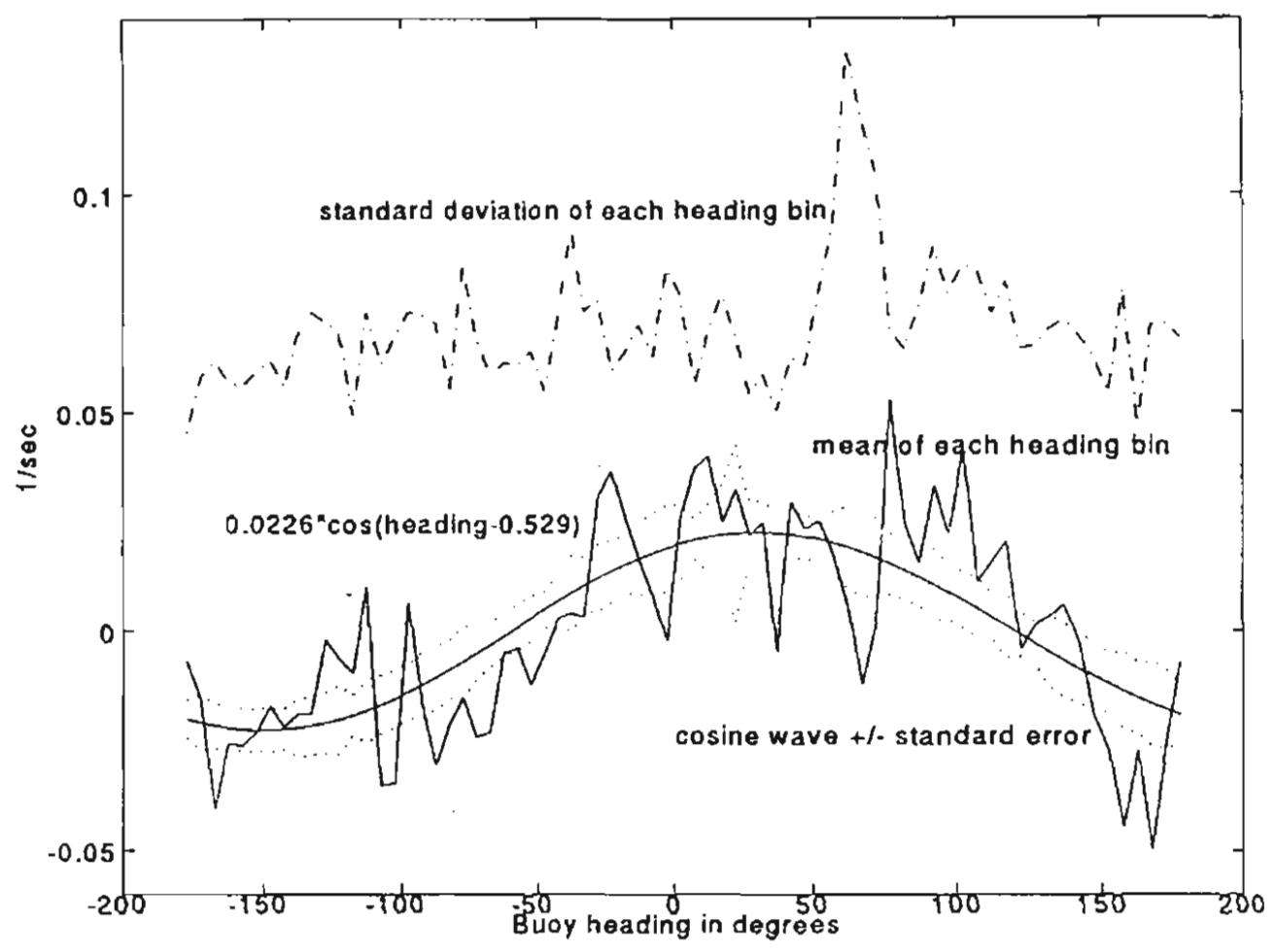

Fig. 3-29. Horizontal vorticity in buoy coordinates, averaged in buoy heading bins, along with the standard deviations of vorticity and the 20 minute mean vorticity rotated into buoy coordinates for each heading plus and minus the standard error.

horizontal vorticity had an autocorrelation time scale of one sample. The bin averaged vorticity means in buoy coordinates do not show any statistically significant biases (differences from the sine wave resulting from the mean earth-referenced vorticity rotated into buoy coordinates) that vary with heading. No bias of horizontal-vorticity variation with heading, combined with the radial symmetry of the buoy, indicate that there is no significant down-wave vorticity bias.

No inference of cross-wave vorticity bias can be made from breaking buoy referenced vorticity into heading bins. Recall that cross-wave vorticity corresponds to velocity in the plane of orbital wave motion. Vertical asymmetry of the buoy and vertical asymmetry of wave velocity (wave attenuation with depth) along with free-surface effects prevent an inference of no cross-wave bias that is independent of buoy heading. From Fig. 3-29. we can infer that cross-wave vorticity bias does not change significantly with buoy heading but we cannot infer that there is no cross-wave vorticity bias. 


\section{WAVE SPECTRAL REJECTION OF VORTICITY SENSORS}

This section measures the wave spectral rejection in a laboratory wave tank and in a bottom deployment in the coastal ocean. Buoy flexing causes a measured spectral peak which is measured and explained.

\section{LABORATORY-WAVE SPECTRAL REJECTION}

The prime motivation to measure vorticity was to filter out irrotational, surfacegravity waves. This section measures how well this goal has been met. The wave spectral rejection of the 15-centimeter path vorticity sensor prototype was measured in a small wave tank. Time series of the sensor measurements are shown in Fig. 3-30. The top
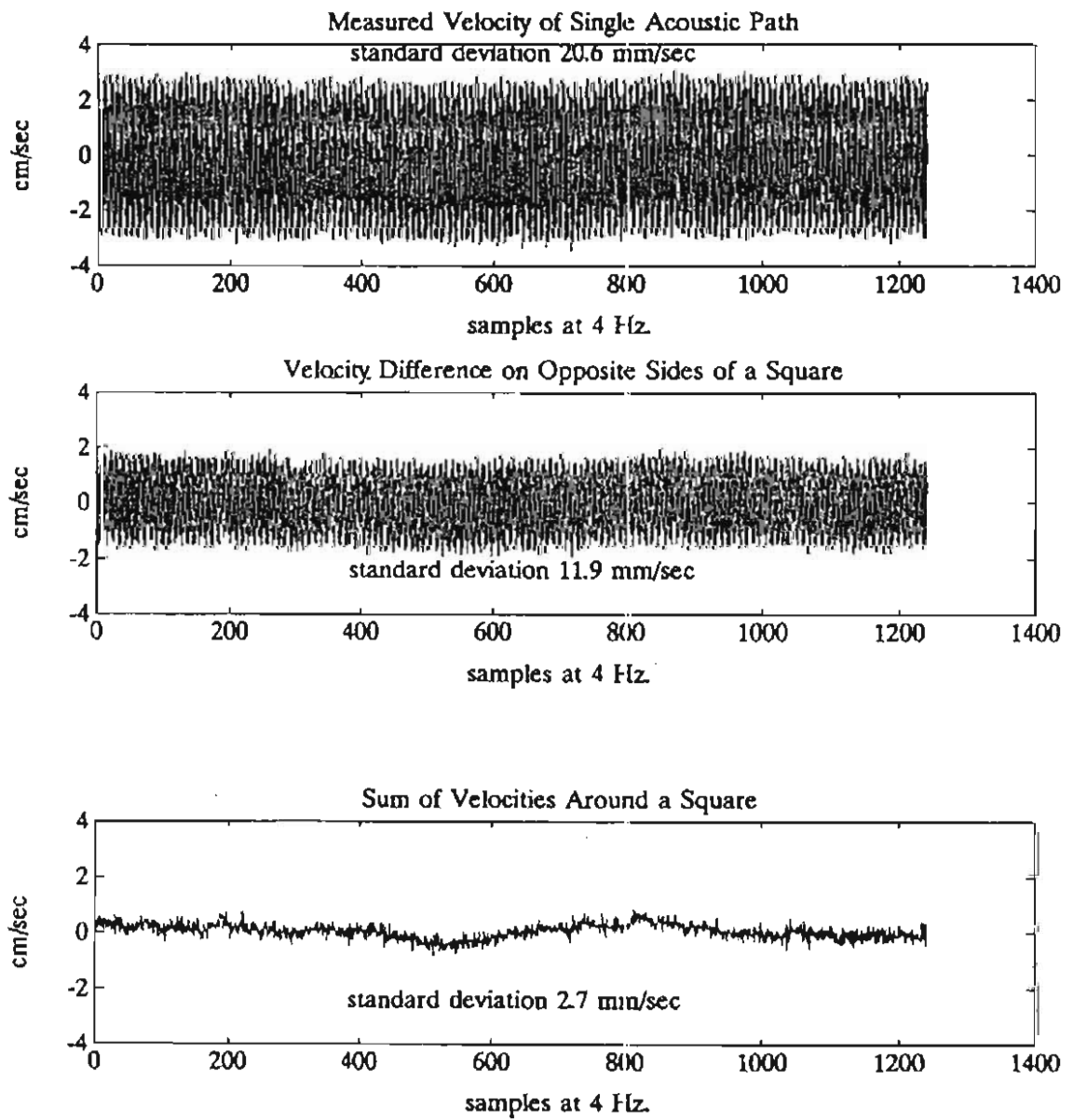

Fig. 3-30. Wave tank time series of velocity, shear, and circulation over path length. 
graph is the measured velocity of one path of a circulation path square and had a standard deviation of $2.06 \mathrm{~cm} / \mathrm{s}$. The middle graph is the measured velocity difference between two parallel paths of the circulation path square and had a standard deviation of $1.2 \mathrm{~cm} / \mathrm{s}$. the bottom graph is the circulation divided by the path length which had a standard deviation of $0.27 \mathrm{~cm} / \mathrm{s}$. Low frequency internal waves and turbulence were evident from the circulation trace that required low-pass filtering of the velocity data to be evident. The same data is plotted in the frequency domain in Fig. 3-31. The sensor has a better than 20 $\mathrm{dB}$ rejection of wave velocity. Most of the noise floor in this spectrum is residual turbulence in the tank from waves, the wavemaker, and sensor wakes. The electronic noise floor in this measurement was $1.8 * 10^{-4} \mathrm{~cm}^{2} / \mathrm{s}^{2} \mathrm{~Hz}$. The 45 -centimeter path vorticity

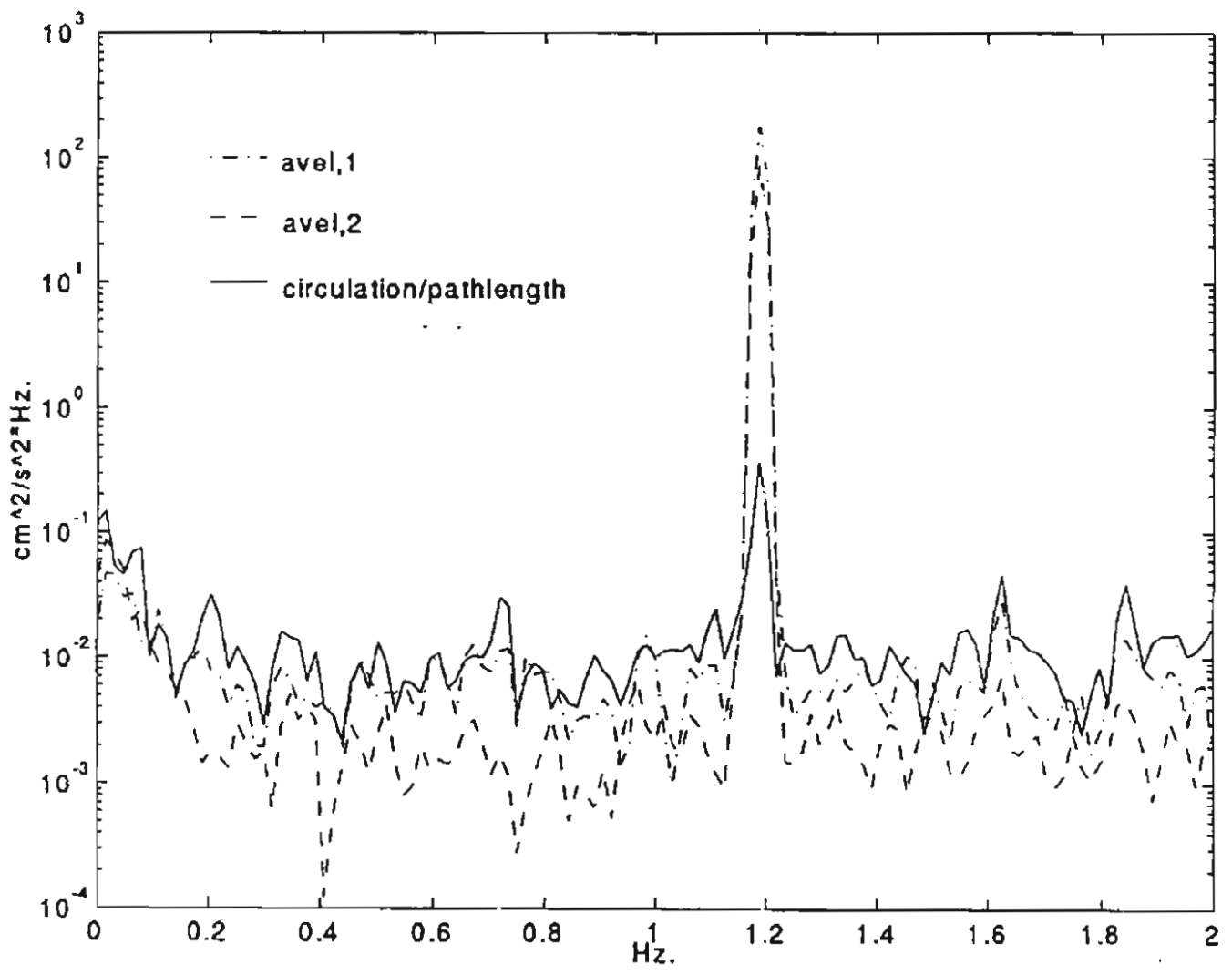

Fig. 3-31. Vorticity meter spectral rejection of waves, avel, 1 and avel, 2 are two acoustic velocity paths of the circulation square.

sensors rejected surface-wave velocities by $30 \mathrm{~dB}$. 


\section{OCEAN-WAVE SPECTRAL REJECTION}

A bottom deployment of the 1.5-meter path vorticity sensor shows its spectral wave rejection. In May of 1994, the instrument was deployed near Woods Hole, MA., in an area of Vineyard Sound having large tidal currents. The data shown in Fig. 3-32 were measured during a storm with surface swell that reached the bottom.

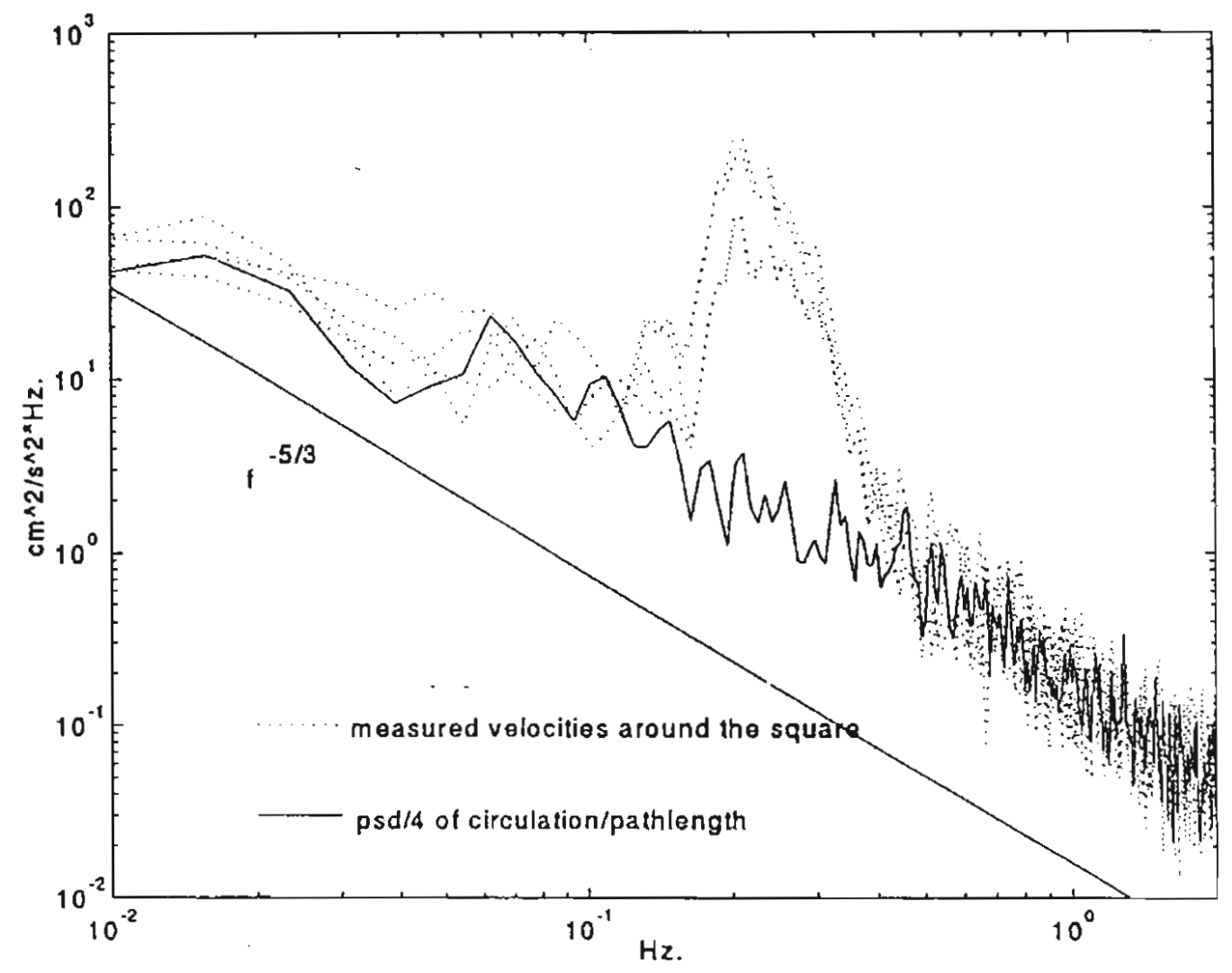

Fig. 3-32. $1.5 \mathrm{~m}$ benthic vorticity meter power spectral densities of acoustic path water velocities and circulation divided by the sum of the path lengths

The measuring volume was centered at 1.75 meters above the bottom. During the time period from which these spectra were computed, the mean current was $22.2 \mathrm{~cm} / \mathrm{s}$ and the rms horizontal wave velocity at the sensor was $5.5 \mathrm{~cm} / \mathrm{s}$. The power spectral densities of the velocities of the individual acoustic paths making up a square are shown as dotted lines and the power spectral density of circulation is shown as solid lines. The power spectral density of circulation over path length has been scaled by 0.25 to give the same energy at 
high frequencies as the velocity spectra. These data were reported by A. J. Williams, Terray, Thwaites, and Trowbridge. (1994). This instrument's effective surface wave rejection shows the utility of measuring vorticity.

The spectra shown in Fig. 3-32 are from time series and cannot be converted to a wave number spectra by the usual frozen turbulence model. The usual test for when the frozen turbulence hypothesis can be used, of the velocity standard deviation being less than half the mean, is not met (Stull, 1989). These sensors measure an area averaged vorticity, not point vorticity. When the frozen turbulence assumption can be used to transform frequency spectra into wavenumber spectra, for eddies with a spatial scale more than twice the vorticity meter size, the spectral transfer function should be one. For eddies with spatial scales smaller than twice the sampling volume, line averaging and area averaging will cause attenuation. For eddies with spatial scales less than a third the path length, the velocity paths will be uncorrelated and should have a spectral response like a travel-time acoustic velocity meter. There is a tradeoff between spatial resolution of a measurement and the accuracy of that measurement.

\section{BUOY FLEXING}

The double-sensor, vorticity measuring buoy is a tall flexible structure that has a bending resonance that can be seen in deployment data, Fig. 3-33. The buoy was built to minimize drag in the measurement volumes by keeping structural elements as slim as practical. The resonance peak, at just below three Hertz, is in the measured horizontal velocity but not the vertical velocity. This peak is enlarged in Fig. 3-34. Compensating for buoy rotation with the rate-gyro in computing horizontal velocity, cannot account for buoy flexing, leaving this error in measured earth referenced velocity. This flexing resonance could only be reduced by making the structure larger, which would cause more drag and flow disturbance. However, this peak is small compared to wave energy and can be ignored. Deployments of the single sensor buoy did not show this peak. The single 


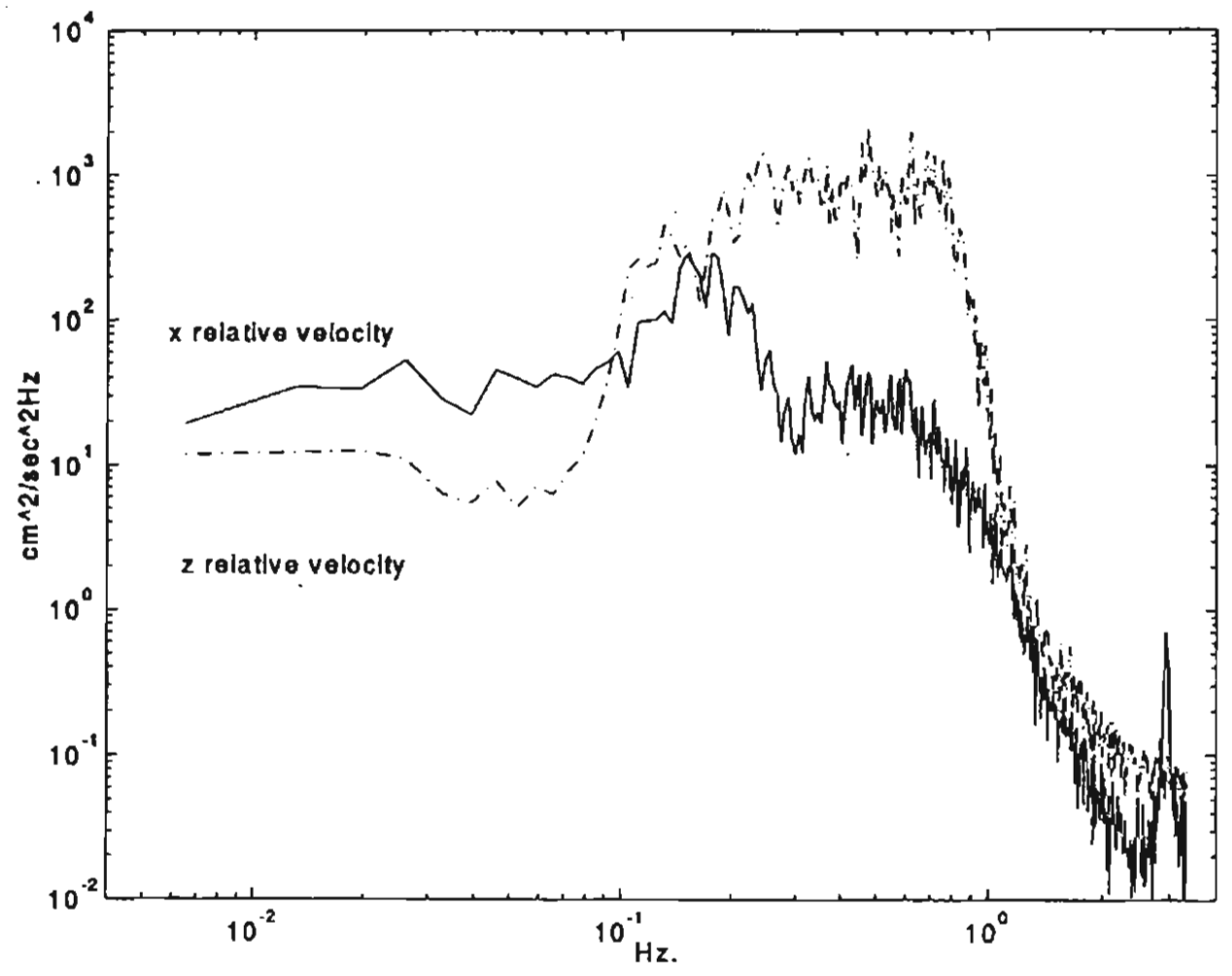

Fig. 3-33. Measured power spectral density of east and vertical velocity. The buoy flex resonance is on the lower right side of the graph.

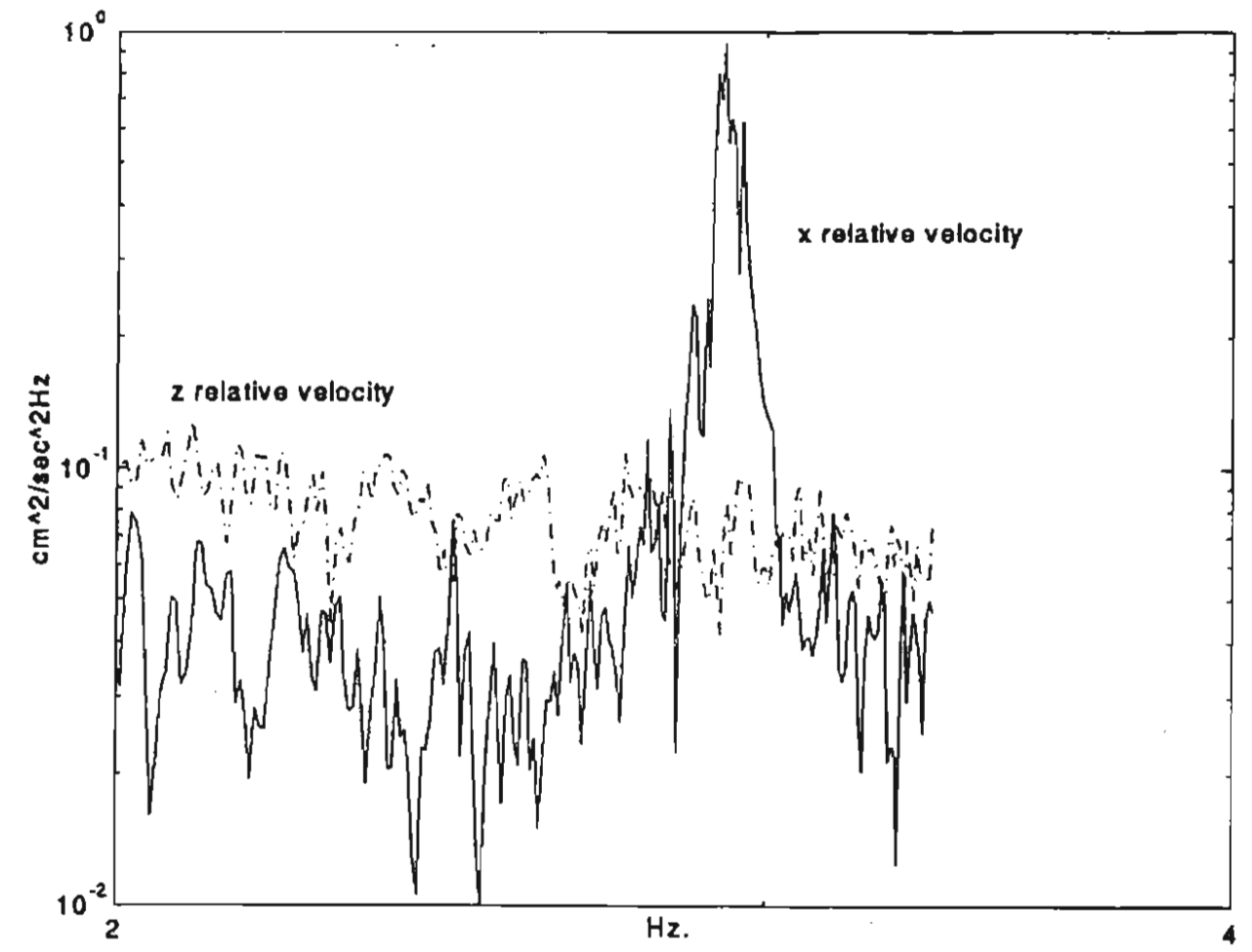

Fig. 3-34. Enlarged view of measured spectral peak. 
sensor buoy has a much higher flexing natural frequency, and at this higher frequency the wave excitation forces are smaller.

This chapter modeled and measured vorticity meter accuracy. A potential flow model was developed that predicted a calibratable reduction in gain but no bias or noise. Wake models were developed to predict bias in constant flow. Electronic noise and bias were shown to be much smaller than flow disturbance related error. Bias in constant flow was found be less than $1 \times 10^{-2} / \mathrm{s}$ with the 45 -centimeter path sensor and variance in constant flow was measured. Bias in wave flow was measured to be less than $1 \times 10^{-2} / \mathrm{s}$ in the down-wave and vertical direction and about $1 \times 10^{-2} / \mathrm{s}$ in the cross-wave direction in open-ocean conditions. Rejection of surface waves was measured and showed the utility of measuring vorticity when a measurement of either turbulence or shear is desired in the presence of waves. The vorticity measuring buoy was shown to be able to make shear measurements in the upper five meters of the ocean over finer resolutions than has been practical before.

The major vorticity sensor error sources are reviewed along with how the errors scale with sensor size and whether the error is noise or bias, in Table 3-4. In this table, $d$ refers to the outer diameter of the pod spheres, $D$ refers to the center stalk diameter, and $L$ refers to an acoustic path length. The transducer pod diameters for the $15 \mathrm{~cm}$ path and 45 $\mathrm{cm}$ path vorticity meters are $7 / 8$ inch $(2.22 \mathrm{~cm})$ and $11 / 8$ inch $(2.86 \mathrm{~cm})$ and the center tube diameters are $1 / 2$ inch $(1.27 \mathrm{~cm})$ and $1 \frac{1}{2}$ inch $(3.81 \mathrm{~cm})$ respectively. 
FLOW ERROR

SOURCES

Electronic noise

Zero drift

Sound speed

Potential flow

Wake 2D

center strut wake

Wake 3D

pod wake

Lift related

Advection of wake

Vortex shedding
VELOCITY VORTICITY NOTES

SCALE

SCALE

$1 / \mathrm{L}$

$1 / \mathrm{L}^{2}$

noise, $\mathrm{rms} 0.1 \mathrm{~mm} / \mathrm{s}$

$1 / \mathrm{L}$

$1 / \mathrm{L}^{2}$

$\left(c_{a}^{2}-c_{r}^{2}\right) / c_{a}^{2} \times$ gain

change in gain

$1^{\circ} \mathrm{C}-0.6 \%$ error in

scale, can be

compensated

reduction in gain

$7.4 \%$ for prototype

$\theta=0$

$\frac{U_{\infty} D \tan \theta}{L} \frac{U_{\infty} D \tan \theta}{L^{2}}$ bias

$U_{\infty}\left(\frac{d}{L}\right)^{\frac{4}{3}} \quad \frac{U_{\infty}}{L}\left(\frac{d}{L}\right)^{\frac{4}{3}} \quad$ bias

$\mathrm{U}_{\infty} \mathrm{D} / \mathrm{L}$

$\mathrm{U}_{\infty} \mathrm{D} / \mathrm{L}^{2}$

bias

function of wave field and sensor motion

bias and noise $0.01 / \mathrm{s}$ in 28 $\mathrm{cm} / \mathrm{s}$ relative velocity

$1.5 \times \mathrm{U}_{\infty} \mathrm{D} / \mathrm{L} \quad 1.5 \times \mathrm{U}_{\infty} \mathrm{D} / \mathrm{L}^{2} \quad$ noise

Table 3-4 Major sensor error sources 


\section{CHAPTER 4. APPLICATIONS}

Chapter four describes two deployments of the vorticity measuring buoy to demonstrate the potential of the instrument to measure shear and vorticity over finer resolutions than before practical. The first application measured shear in the upper-ocean boundary-layer within 5 meters of the surface. The second application measured gradient Richardson number with fine resolution in a thermocline. An application of measuring turbulence with a vorticity meter in the bottom-boundary layer was described in the Ocean-Wave Spectral Rejection section.

\section{A. UPPER-BOUNDARY-LAYER SHEAR}

The first application of the double 45-centimeter path, shear-measuring buoy to be discussed is measurement of shear in the upper-ocean, boundary layer. Shear, turbulence, and mixing in unstratified, turbulent, boundary layers over rigid walls have been well studied and are well understood. By comparing the shear in an ocean boundary layer to the stress over this layer, the effect on mixing of the free surface, stratification, and possible organized motions like Langmuir circulation can be measured. The shear expected in the log layer next to a rigid wall is given by equation (4-1), where $d U / d z$ is the

$$
\frac{d U}{d z}=\frac{u_{\dot{*}}}{\kappa z}
$$

shear, $u_{0}$ is the friction velocity (the square root of the shear stress divided by density), $\kappa$ is von Karman's constant and is usually assumed to be 0.4 , and $z$ is the distance from the wall.

The shear-measuring buoy was deployed in Buzzards Bay, Massachusetts, in May 1994. The measured shears are compared to the shear one would expect in an unstratified, rigid wall, turbulent flow of the same shear stress, Fig. 4-1 (Monin and Yaglom, 1982). The wind stress was concurrently measured by a sonic anemometer and 
calculated using the inertial dissipation method (Fairall and Larsen, 1986). The wind during the deployment increased in strength from about $5.2 \mathrm{~m} / \mathrm{s}$ to about $15.2 \mathrm{~m} / \mathrm{s}$, causing the water-side friction velocity to increase from $0.62 \mathrm{~cm} / \mathrm{s}$ to $1.82 \mathrm{~cm} / \mathrm{s}$. The error bars
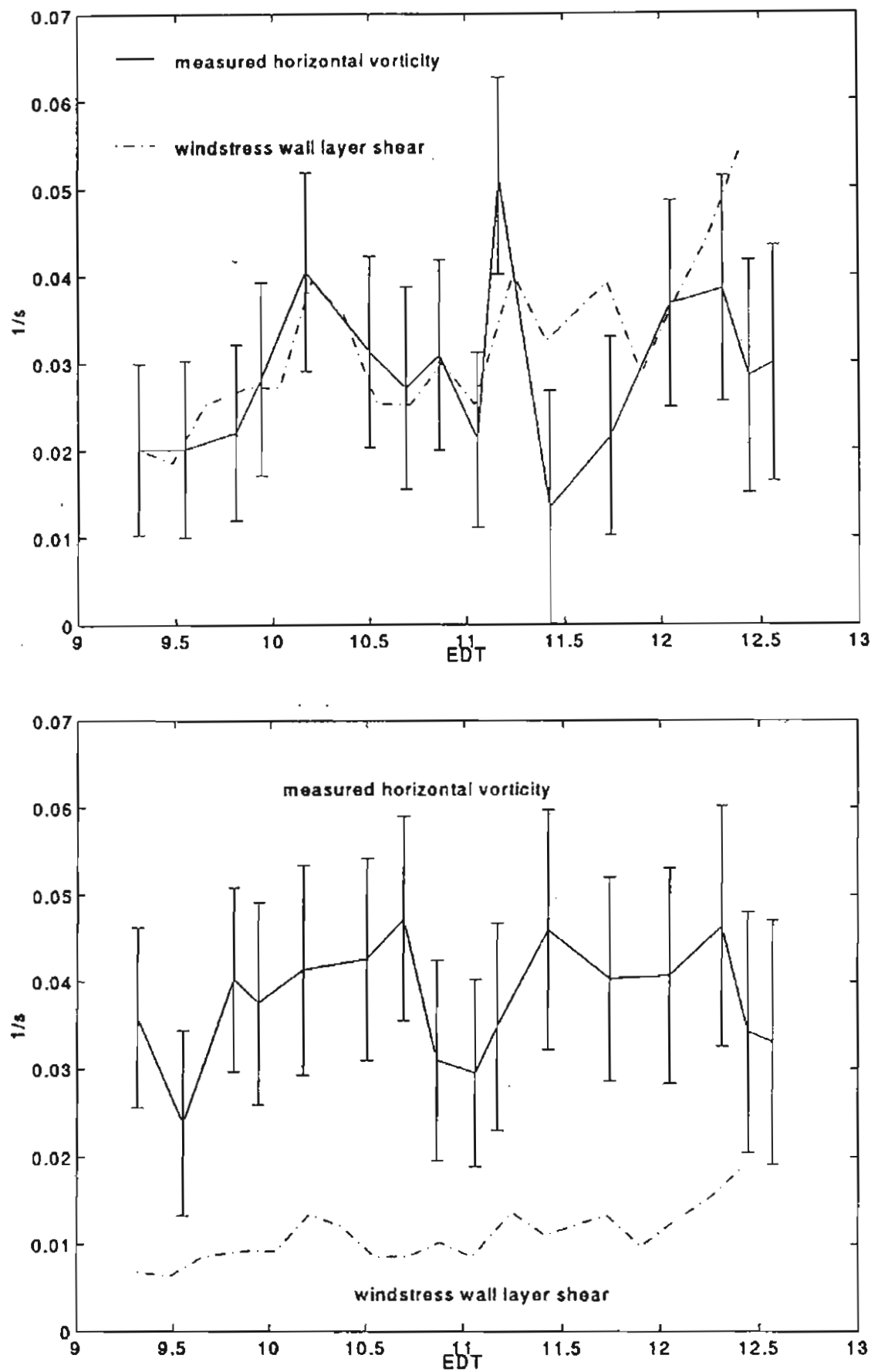

Fig. 4-1. Buzzards Bay deployment shears measured and estimated from wind stress. The upper graph is the sensor at $0.83 \mathrm{ml}$ depth and the lower graph is the sensor at $2.45 \mathrm{~m}$ depth. 
are derived, as described in the Bias in Wave Flow section, from an open-ocean deployment with significant swell and negligible wind stress, and are scaled with rms velocity relative to the sensors. The sensor at the shallower depth of 0.83 meters, measured shear that coincided with the log-layer shear prediction for most of the deployment. Toward the end of the deployment, as the windstress, waves, and wave breaking increased, the shear started to drop and diverge from the wall model. The vertical heave velocity spectra at the start and end of the deployment are shown in Fig. 42. The vertical heave velocity variance increased from $100 \mathrm{~cm}^{2} / \mathrm{s}^{2}$ at the start to 252 $\mathrm{cm}^{2} / \mathrm{s}^{2}$ at the end.

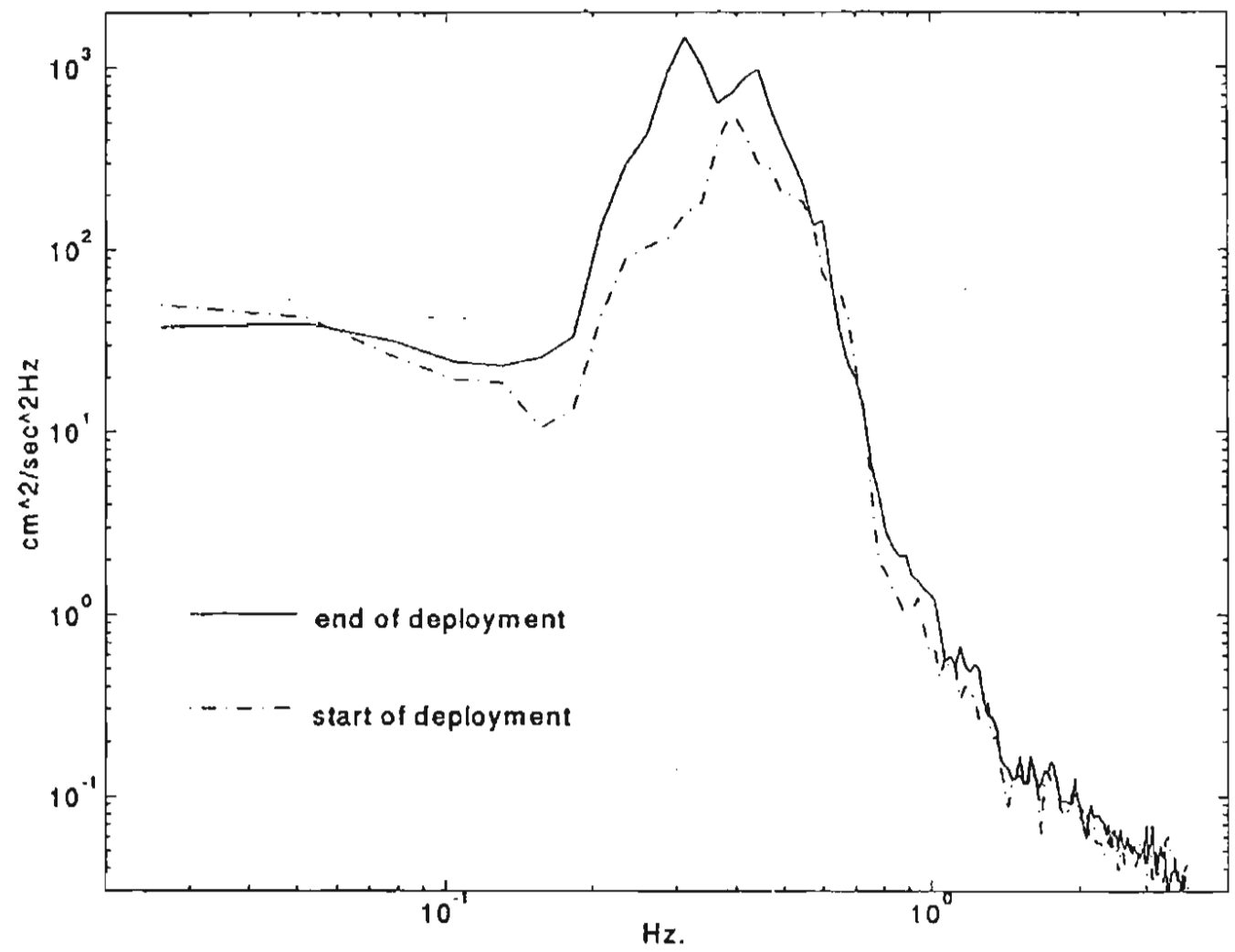

Fig. 4-2. Vertical velocity spectra at the start and end of the Buzzards Bay Ma deployment. The heave velocity variance increased from $100 \mathrm{~cm}^{2} / \mathrm{s}^{2}$ to $252 \mathrm{~cm}^{2} / \mathrm{s}^{2}$. 
The sensor at the deeper average depth of 2.45 meters, measured about three times more shear than would be predicted by a log layer. Average temperature stratification measured at 2.45 meters depth gave a Brunt-Vaisala frequency of $0.012 / \mathrm{s}$ (period of about nine minutes), and the measured gradient Richardson number at this depth dropped from 0.20 to 0.10 . Stratification was inferred from temperature assuming that salinity was well mixed in the upper five meters. Surveys of stratification done with a conductivity, temperature, depth instrument (CTD) in this part of Buzzards Bay during this time of year have shown salinity to be well mixed over the upper five meters and the error in stratification resulting from not measuring salinity should be less than twenty percent. The Richardson number time series are plotted in Fig. 4-3. These are reasonable Richardson

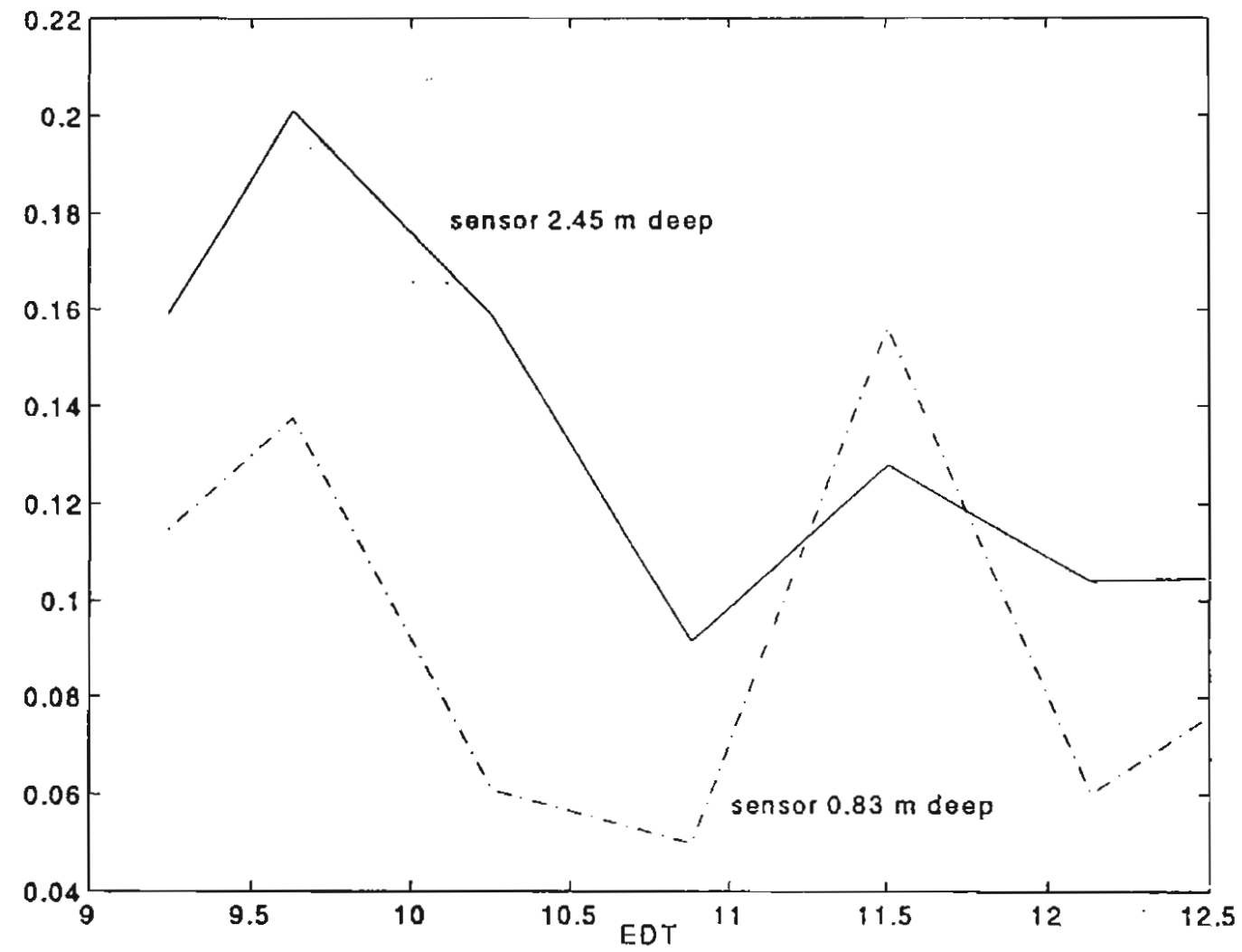

Fig. 4-3. Gradient Richardson numbers measured during the Buzzards Bay deployment.

numbers for a stressed boundary layer with turbulent mixing. A more detailed vector 
breakdown of these results into directions downwind and crosswind is in Appendix C. The increased shear of the sensor at 2.45 meters as compared to the predicted shear of an unstratified boundary layer is a measurement of the stratification's inhibition of vertical turbulent mixing and consequent enhancement of shear.

The measured data from this deployment will now be replotted in vector components along with shears to show the sensor-wave-correlation bias. As the SensorWave-Correlation Bias section described, velocity or shear derived from velocity measured from a platform that moves in a correlated way with the waves, can have a bias that can be as large as the Eulerian shear. The vorticities and shears are plotted with respect to the dominant wave direction. The measured wave velocity directional spectrum calculated by the maximum entropy method is plotted in Fig. 4-4. The wind was from

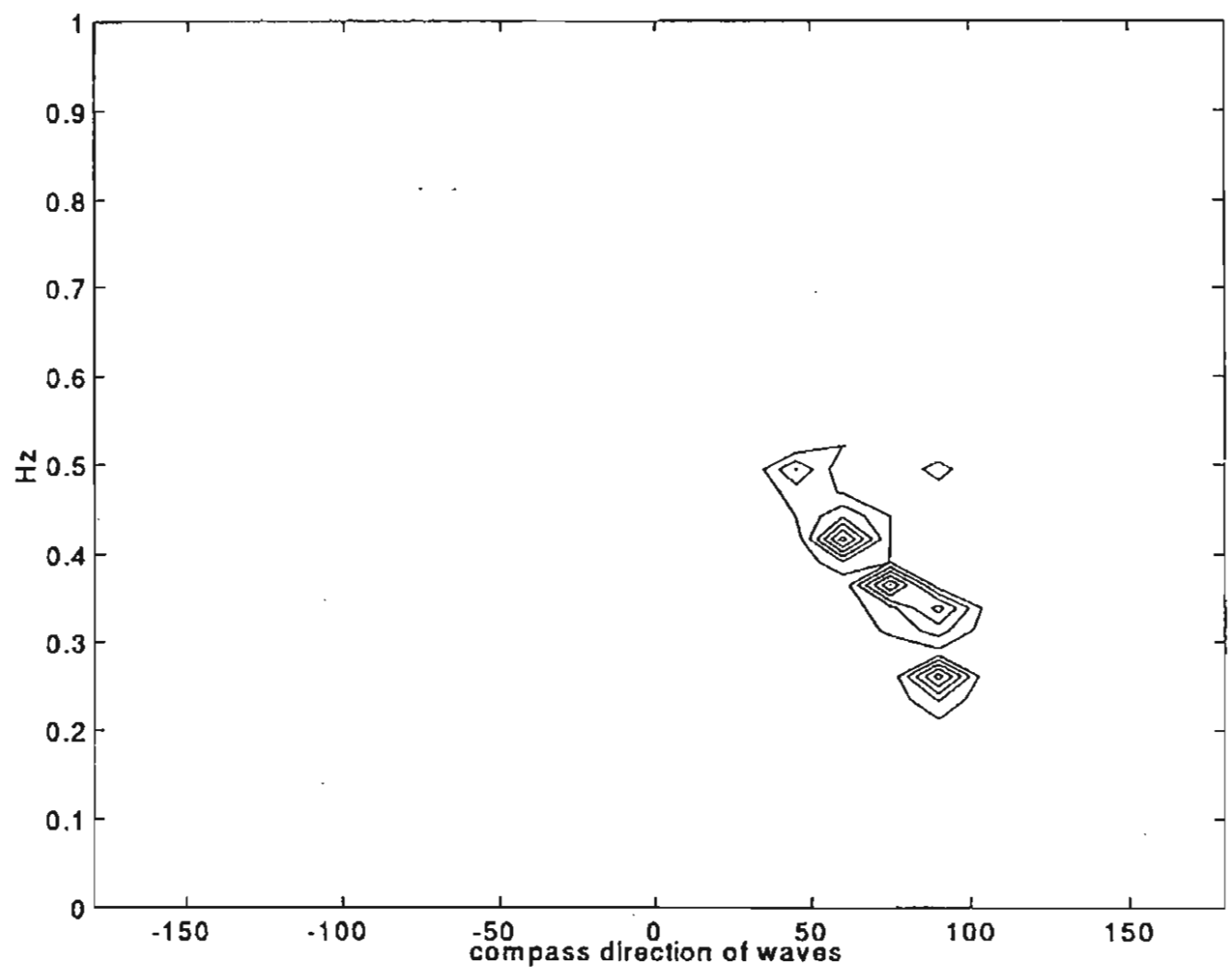

Fig. 4-4. Wave velocity directional spectrum calculated by the maximum entropy method for the Buzzards Bay deployment. 
$205^{\circ}$ but the bay is oriented east-west on a magnetic compass causing the longer period waves to be from the west. The shorter period waves were from the southwest. The peakiness of the directional spectrum may be an artifact of the maximum entropy method; both the heave spectrum and the Longuet-Higgins spectrum show only two peaks.

The vorticity and shear measured by the shallow sensor at 0.83 meters are plotted in Fig. 4-5 and by the deeper sensor at 2.45 meters in Fig. 4-6. These are plotted in east and north coordinates that correspond to the longer period waves. Because all the waves were not from the same direction, it was not possible to rotate the measurements to correspond with all the waves. The shears measured were from the velocity paths on the top and bottom of the vorticity measurement volumes and in the shallow sensor measure

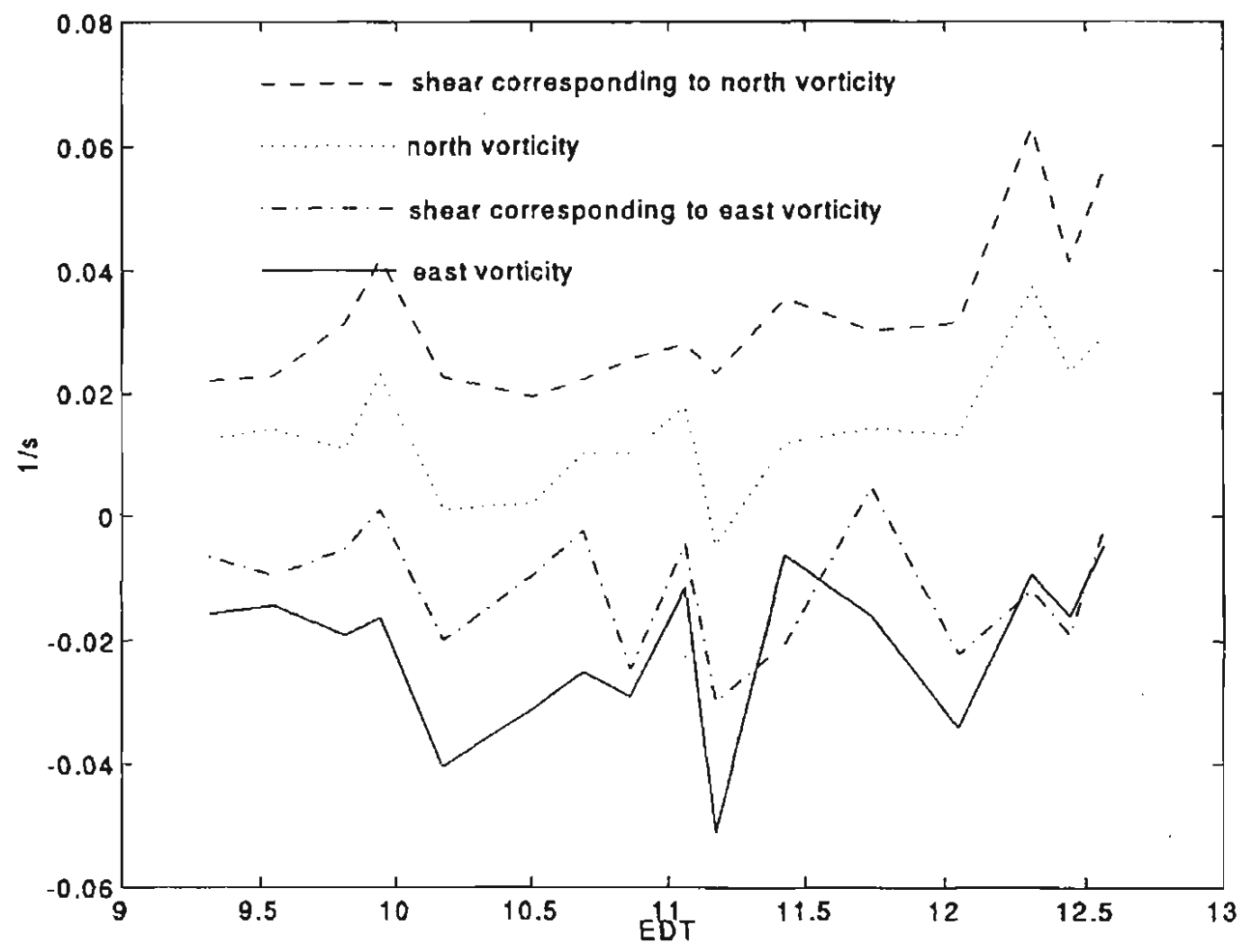

Fig. 4-5. Vorticity and shear corresponding to that direction of vorticity, measured by the sensor $0.83 \mathrm{~m}$ deep during Buzzards Bay deployment. 
significantly more shear in the down-wave direction. The shallow sensor's shear corresponding to the north vorticity, was $1.8 \times 10^{-2} / \mathrm{s}$ greater than north vorticity. This corresponds with the predominant waves radiating east and is a measurement of correlation-wave bias. Shear and vorticity in the other direction and measurements in the lower sensor have differences that are smaller than the error bars but, in all but the lowersensor, east vorticity, are of the right sign. The lower-sensor, east vorticity only differs from its corresponding shear by $0.2 \times 10^{-2} / \mathrm{s}$. Removing correlation-wave bias is important when measuring shear from buoys in the upper five meters of the ocean, and this instrument does so intrinsically.

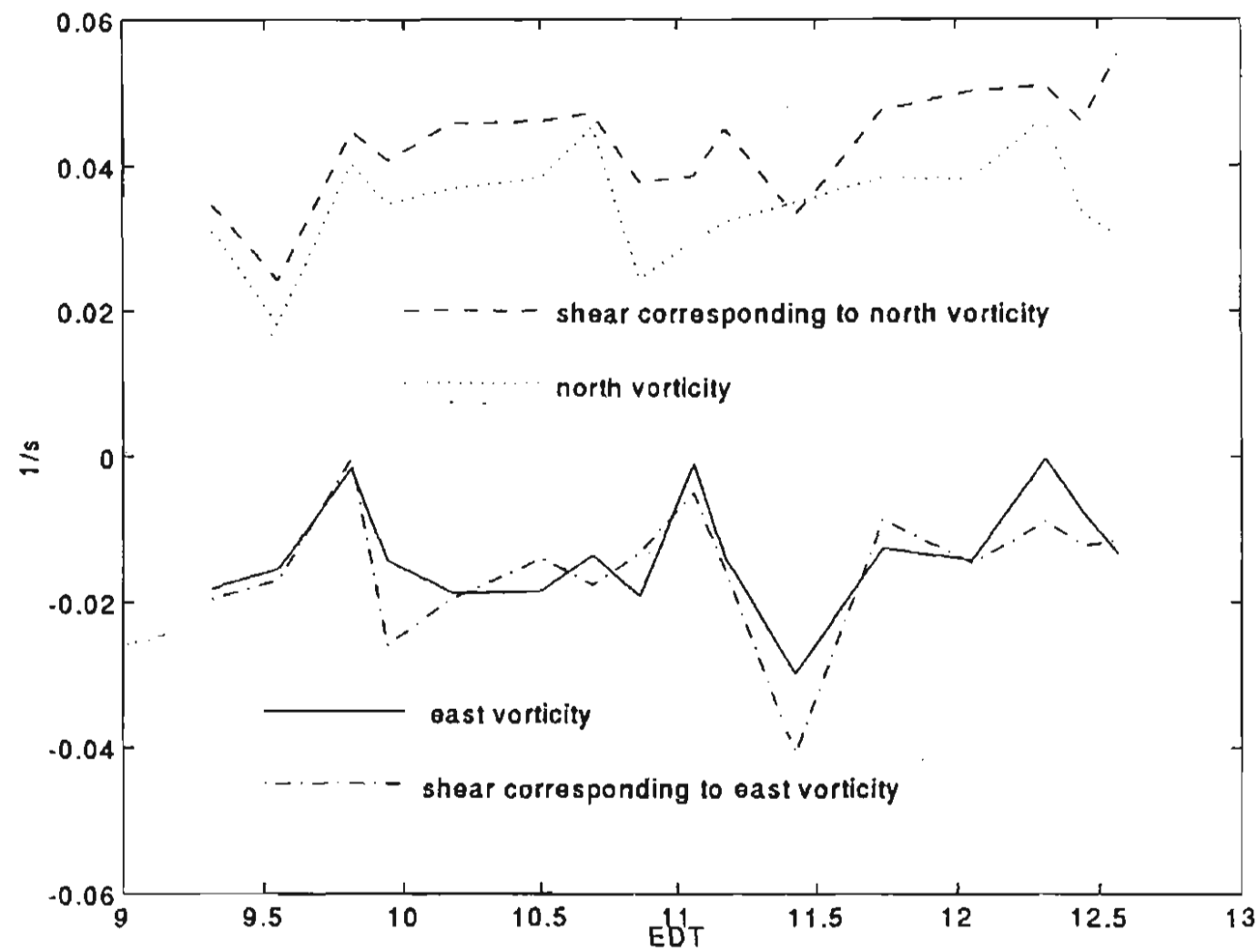

Fig. 4-6. Vorticity and shear corresponding to that direction of vorticity, measured by the sensor $2.45 \mathrm{~m}$ deep during Buzzards Bay deployment. 


\section{B. THERMOCLINE RICHARDSON NUMBER}

The second application of the shear measuring buoy to discuss is measurement of shear and local shear instability in an internal boundary layer. In August of 1993, the shear measuring buoy was deployed with a single sensor in the thermocline in Massachusetts Bay. Fig. 4-7 shows a typical time history of temperature, magnitude of vertical shear, and gradient Richardson number.

The histogram of the gradient Richardson number is shown in Fig. 4-8. In this figure, the histogram of the Richardson number measured over a 1.0-meter bin size is also shown. The latter measurements were made the same day using an acoustic doppler current profiler (ADCP) to measure shear and a conductivity temperature depth instrument (CTD) to measure stratification. The averaging time for the $1.2 \mathrm{MHz} \mathrm{ADCP}$ was 30 seconds for Fig. 4-8, and the shear for both Figs 4-7 and 4-8 was lowpass filtered with a 25 -second period filter. There is a greater proportion of low Richardson number measurements at the smaller vorticity meter 38.6 centimeter resolution than the ADCP 1.0 meter resolution. This difference is in qualitative agreement with the theory by Desaubies and Smith (1982) that predicts more small mixing events than large mixing events in a random, internal-wave field. Twenty-five percent of the Richardson numbers measured over the 38.6-centimeter volume were unstable while $12.7 \%$ of the Richardson numbers measured over the one meter volume were unstable. The Richardson number measurements were made in the middle of a dye tracer release experiment that measured a vertical diffusivity of $0.06 \mathrm{~cm}^{2} / \mathrm{s}$ (Geyer and Ledwell, 1994). This deployment shows that the instrument can measure shear and local shear instability in ocean internal-boundary layers. 

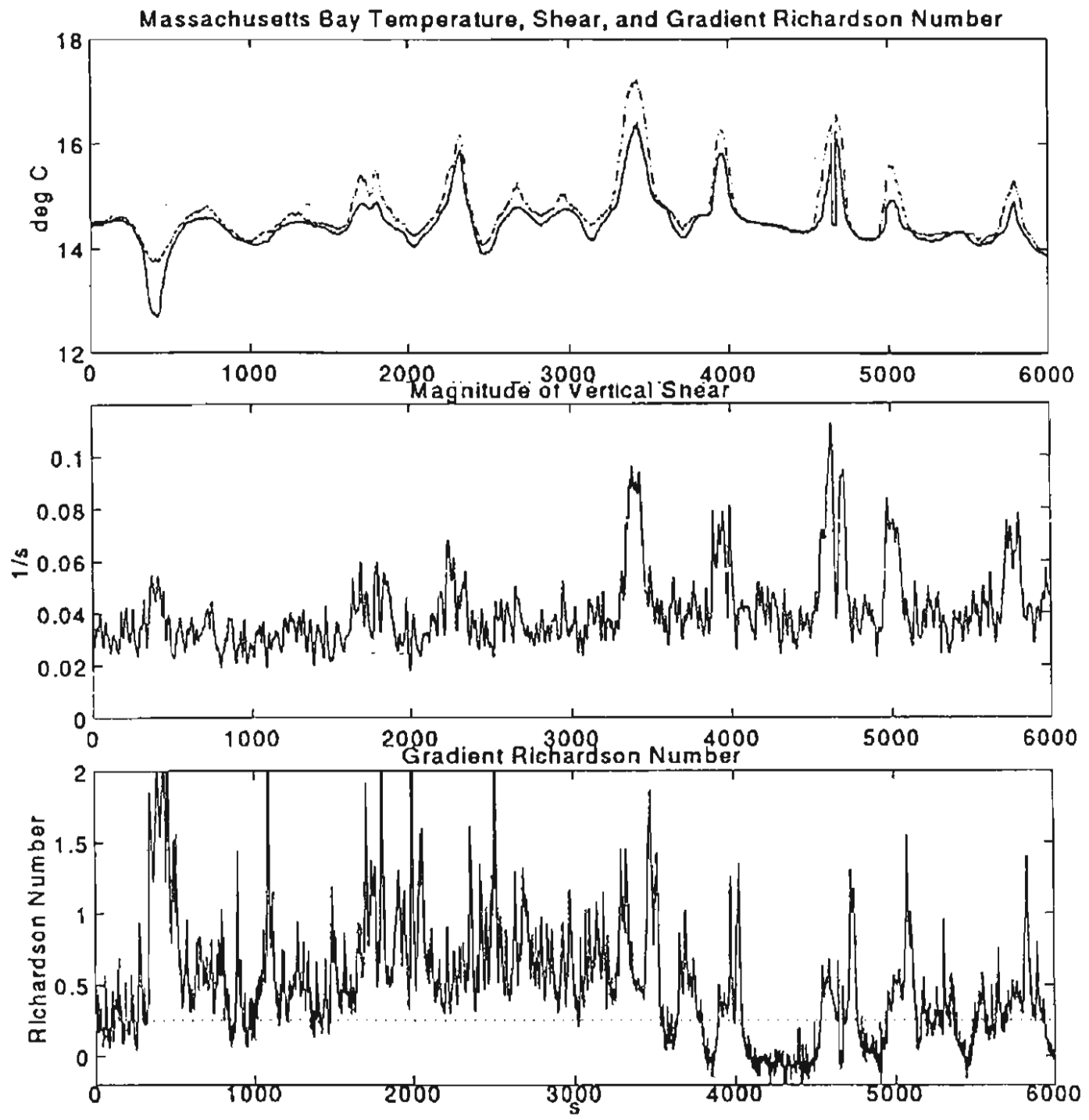

Fig. 4-7. Gradient Richardson number measured in the Massachusetts Bay thermocline. In the top graph of temperature, the dot dash line is the temperature at top of the measurement volume and the solid line is at the bottom. In the Richardson number plot, a dotted reference line is drawn at 0.25 . 


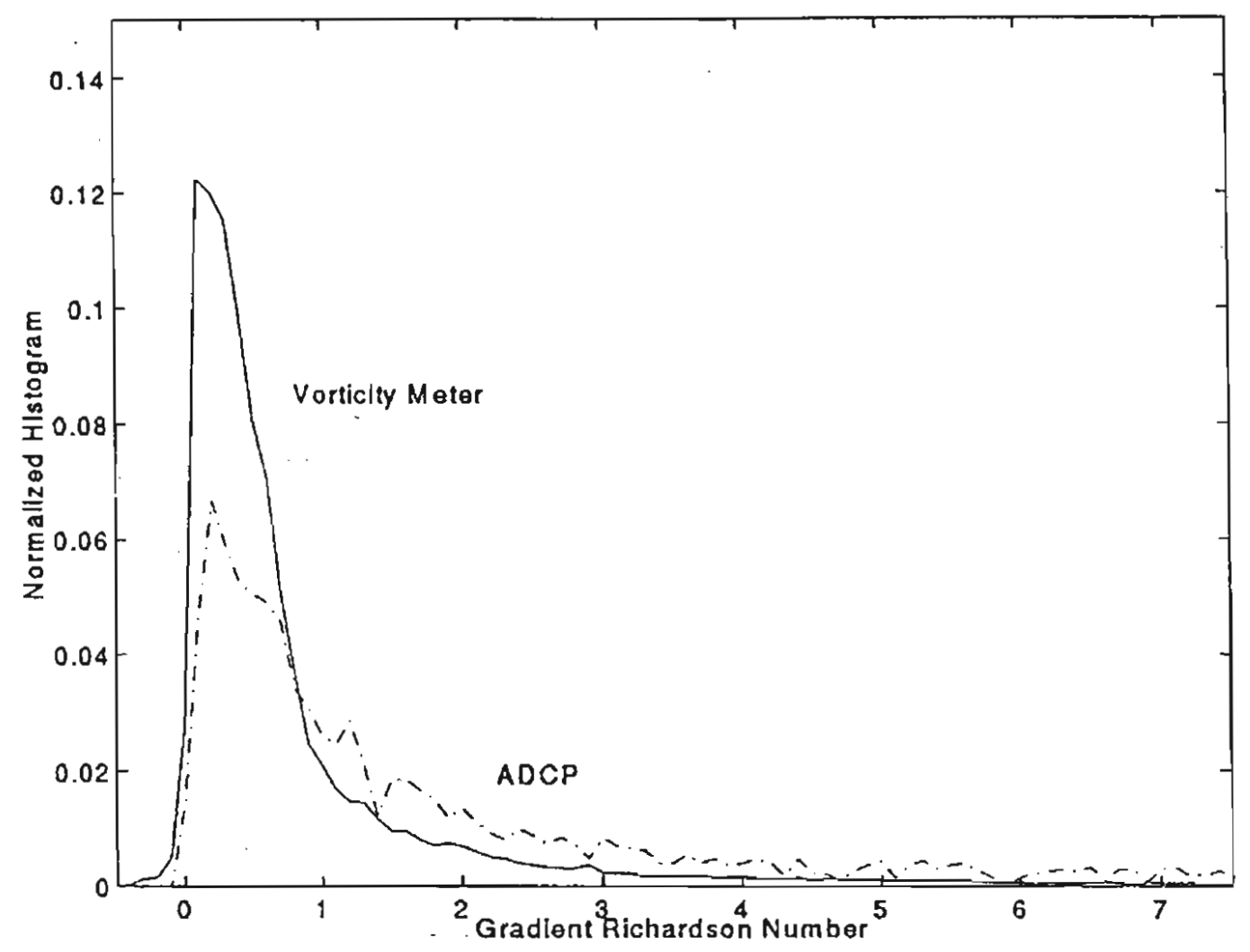

Fig. 4-8. Relative frequency of Richardson numbers measured by the vorticity meter over $38.6 \mathrm{~cm}$ and by an ADCP over $1.0 \mathrm{~m}$. 


\section{CHAPTER 5. SUMMARY}

\section{A. CONCLUDING REMARKS}

The major goal of my research has been to develop an instrument system capable of measuring shear in the upper five meters of the ocean. The data measured and presented in this thesis are a test of the instrument. This is the first, three-axis oceanographic vorticity instrument deployed in the ocean. The design has already been used in a subsequent project to measure coastal, bottom-boundary-layer turbulence in the presence of surface swell.

To remove platform movement from flow measurements, I used a low-power, strapdown, inertial-measurement unit for the instrument system. Constraints of buoy motion allowed the use of an Euler-angle algorithm with complementary-filtering that gave good performance results from slow samples of noisy, drifty, and low-power inertial sensors.

The instrument is suitable for measuring shear in the upper five meters of the ocean, measuring fine scale shear and vorticity in internal-boundary layers, and coastal, bottom-boundary-layer turbulence in the presence of surface swell. Measurement errors are dominated by asymmetries between sensor wakes and circulation paths. This analysis has shown the tradeoff between spatial resolution and accuracy in measuring shear or vorticity.

In the first chapter, I showed the need for measurements of shear in the upper five meters of the ocean, the desirability of filtering out irrotational water motion such as surface gravity waves when measuring shear, and the need to remove or compensate for sensor motion when measuring shear. Sensor-wave-correlation bias of velocity and velocity-derived shear measurements was reviewed and non-intrusive measurements of vorticity were shown to not have this bias. The only reason to average vorticity measurements is to reduce fluctuating wake error effects. 
In the second chapter, I motivated the mechanical design, described the electrical system and inertial measurement unit, reviewed rotation and strapdown inertial computation, described the inertial algorithm used, and described simulations of the inertial system. In measurements of shear or vorticity with this instrument, error contribution from imperfect, inertial compensation is much smaller than wake-related errors.

The third chapter developed models of and described measurements of the instrument's major error sources. Electronic noise and drifts were shown to be much smaller than flow-related errors. The simpler, triangular circulation path was shown to have much greater bias in constant flow than the chosen square circulation path. Variance in constant flow was shown to result from vortex shedding off bluff sensor parts. Simple attempts to streamline the sensor were shown to be capable of increasing instrument biases. Laboratory measurements that simulated waves showed down-wave and vertical biases of less than $1 \times 10^{-2} / \mathrm{s}$, that cross-wave bias varied linearly with $\mathrm{ms}$ relative velocity and did not vary stongly with down-wave drift velocity. Cross-wave vorticity bias in wave flow was measured in an ocean deployment with no wind stress and significant swell to have a bias as large as $1 \times 10^{-2} / \mathrm{s}$. Open-ocean measurements showed that vertical and horizontal vorticity bias did not change significantly with sensor heading. The sensor's spectral surface-gravity-wave rejection was measured in both wave tanks and in the coastal ocean, and showed the utility of measuring vorticity.

The fourth chapter described two ocean deployments of the instrument. Measurements of upper-boundary-layer shear were compared to models of turbulent, boundary-layer behavior. Sensor-wave-correlation bias of velocity-derived shear was measured to be as large as Eulerian Shear. Measurements of the Richardson number in the thermocline over small scales were compared to Richardson numbers measured over larger scales and were consistent with the theory that small, unstable internal wave events are more common than large, unstable events. Data from these deployments showed the vorticity meter's viability in measuring small-scale, ocean-boundary-layer shear. 


\section{B. FUTURE WORK}

The vorticity meter and the shear measuring buoy it assembles into is complete, calibrated, and ready to be redeployed. Uses of the system in future deployments include longer deployments measuring shear over several wind shifts and measurements of Langmuir cells. The vorticity sensor requires shorter averaging times than measurements of shear with velocity meters; this shorter averaging time allows slow traverses across Langmuir cells. The sensor would have to be scaled close to the size of the cells, pathlengths of about a quarter of the cell depth would be appropriate for adequate sampling with good signal-to-noise ratio.

If ocean scientists desired the ability to measure low-frequency wave spectra, possible future system improvements would include installing more accurate rate gyros, sampling faster, and or adding position or velocity measurement with a Kalman filter. 


\section{APPENDIX A.}

Mechanical Drawings

A-1 Shear measuring buoy assembly

A-2 Vorticity sensor assembly

A-3 Pressure vessel assembly

A-4 Lower hub detail

A-5 Upper hub detail

A-6 Custom end cap detail

A-7 Instrument electronics case detail

A-8 Transducer mount detail

A-9 Central stalk, pod arms, and braising jig

The major mechanical drawings for the vorticity sensor are included in appendix $A$. All the drawings scales have been reduced to fit on $81 / 2 * 11$ inch paper. A vorticity sensor of another path length could be readily designed by scaling these drawings. On the assembly drawings, the out of plane pod arms are not shown. The lower end cap is a standard BASS end cap. The braising jig rods are screwed into each acoustic path for braising with screws with modified ends to fit in the tight confines of the pod centers. The dimensions on these drawings are in inches which was required to get reasonable machining bid prices. 


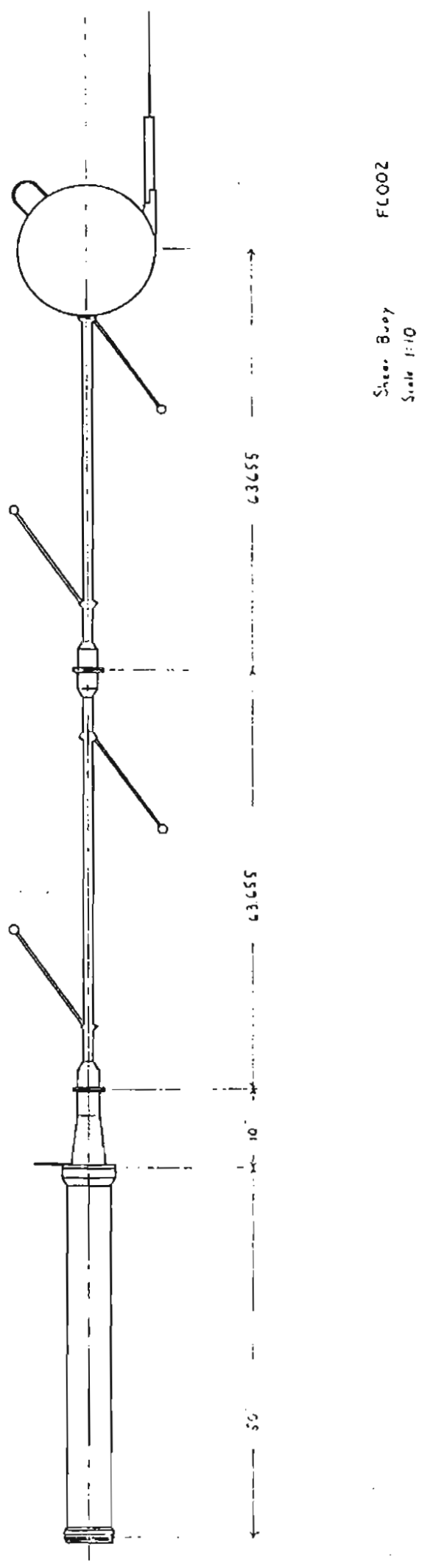

Fig. A-1 Shear measuring buoy assembly drawing 


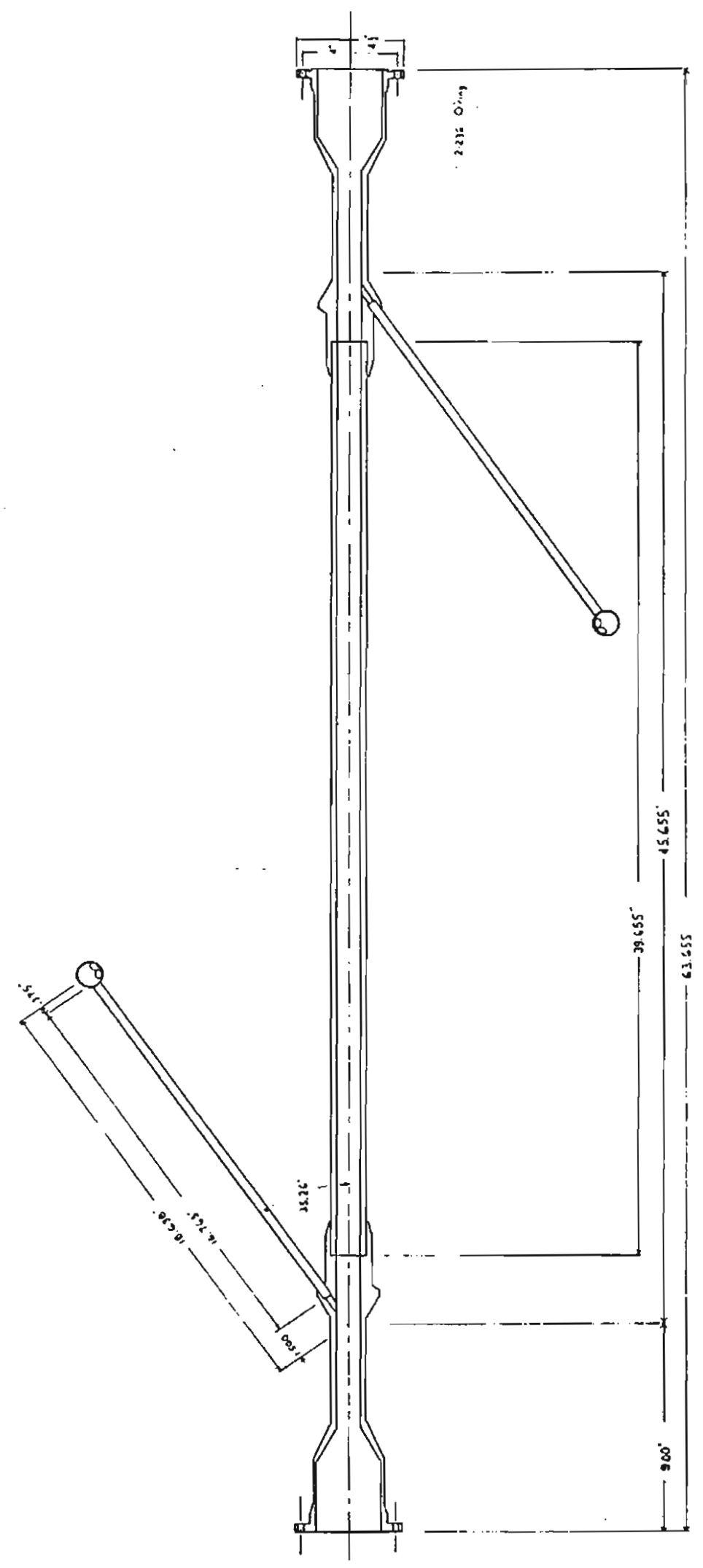

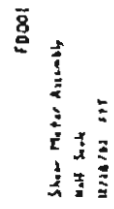

Fig. A-2. Vorticity sensor assembly drawing 


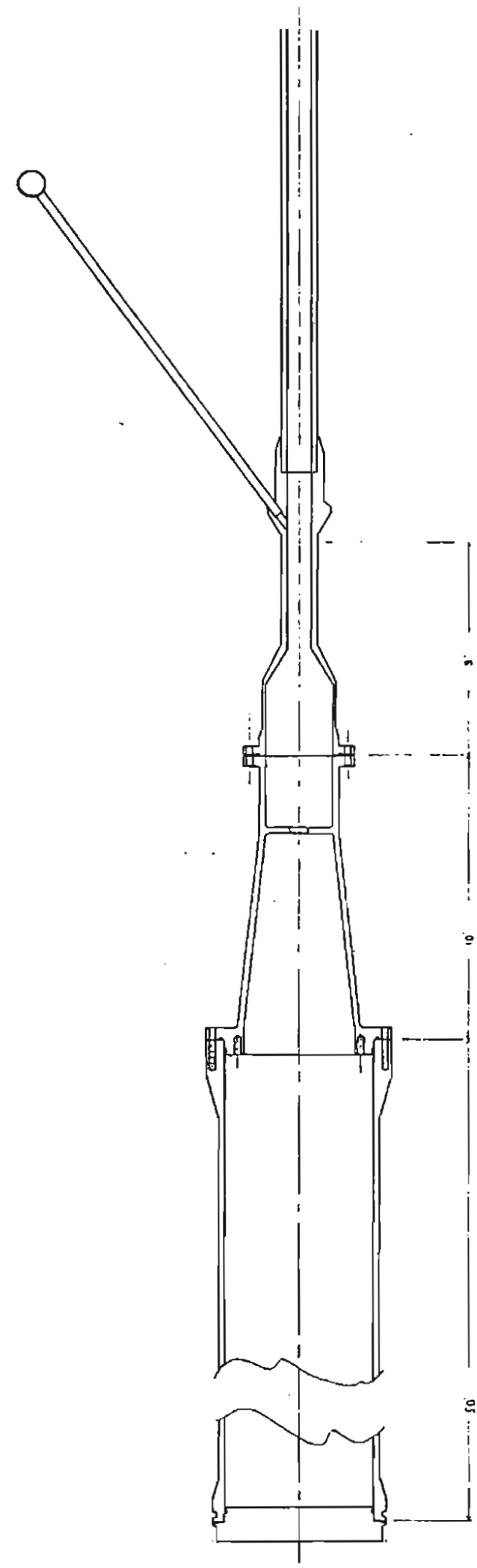

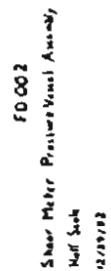

Fig. A-3. Pressure vessel assembly drawing 


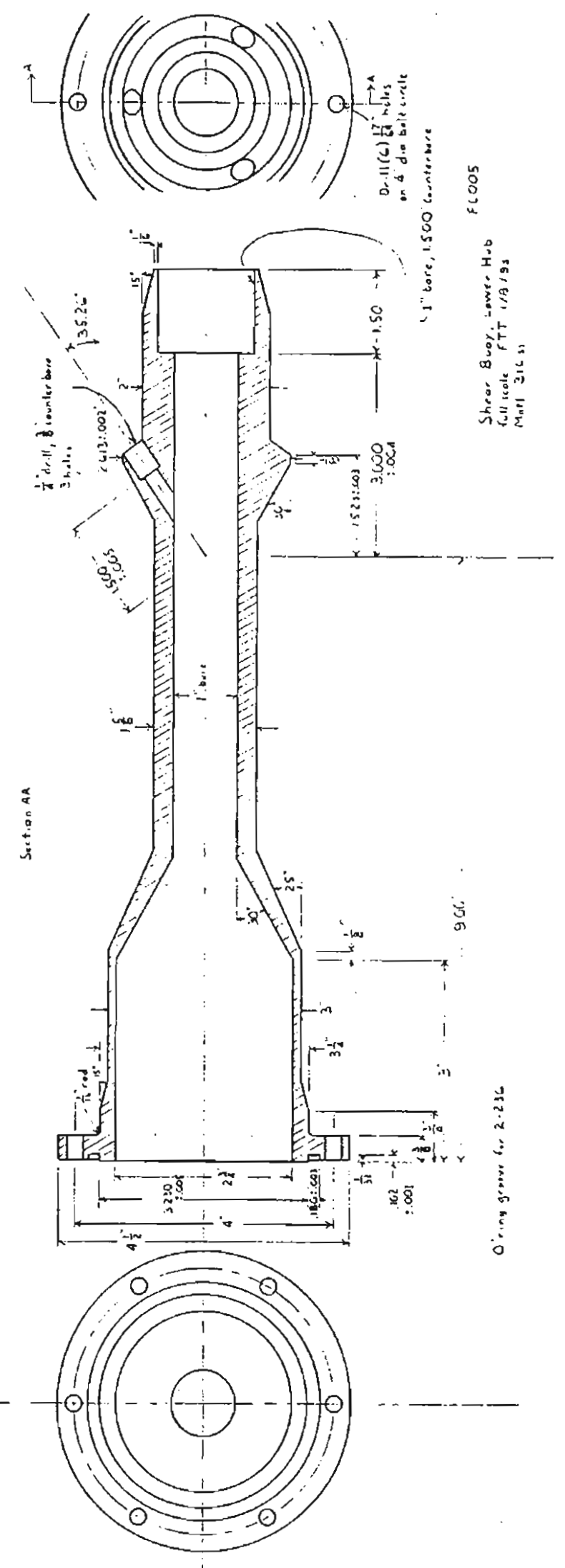

Fig. A-4. Lower hub detail drawing 


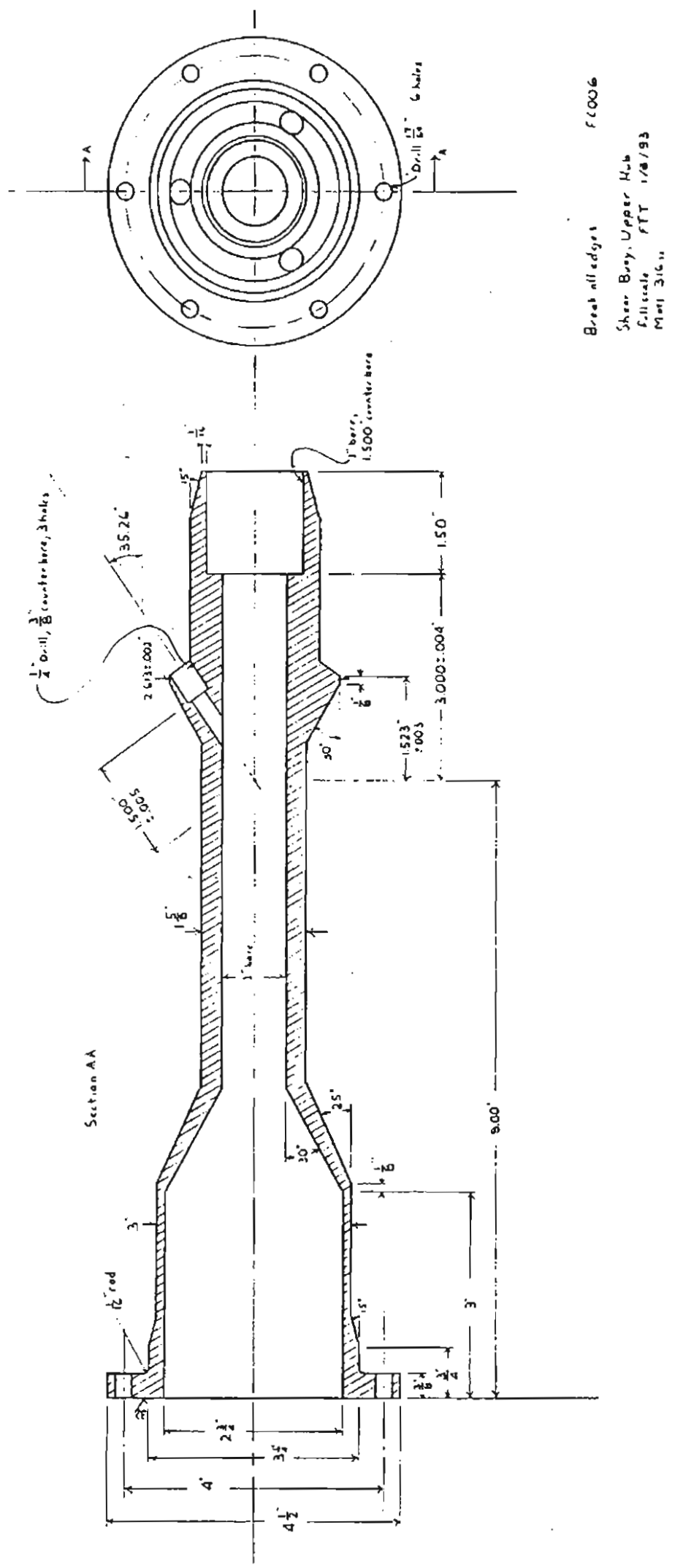

Fig. A-5. Upper hub detail drawing 

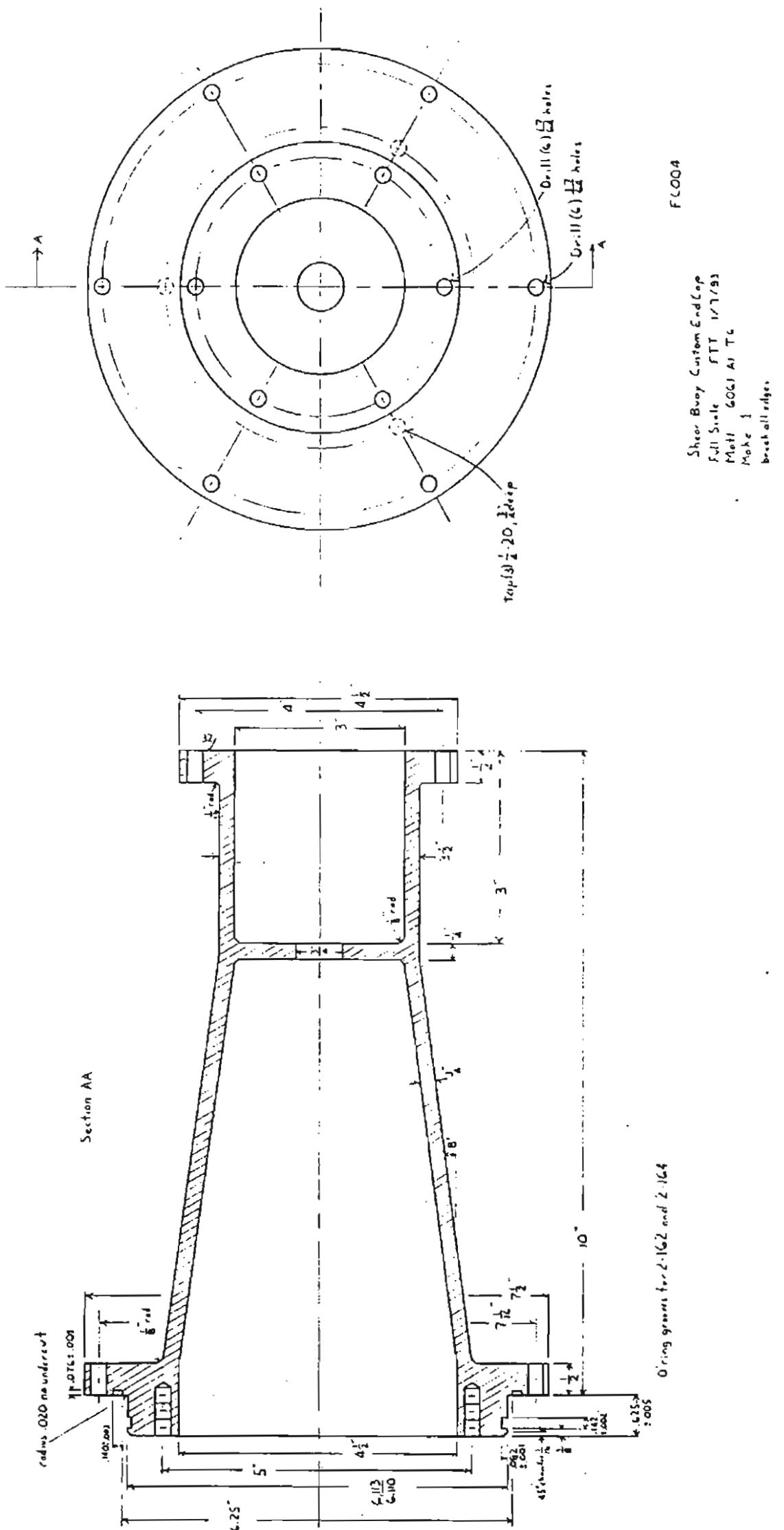

Fig. A-6. Custom end cap detail drawing 


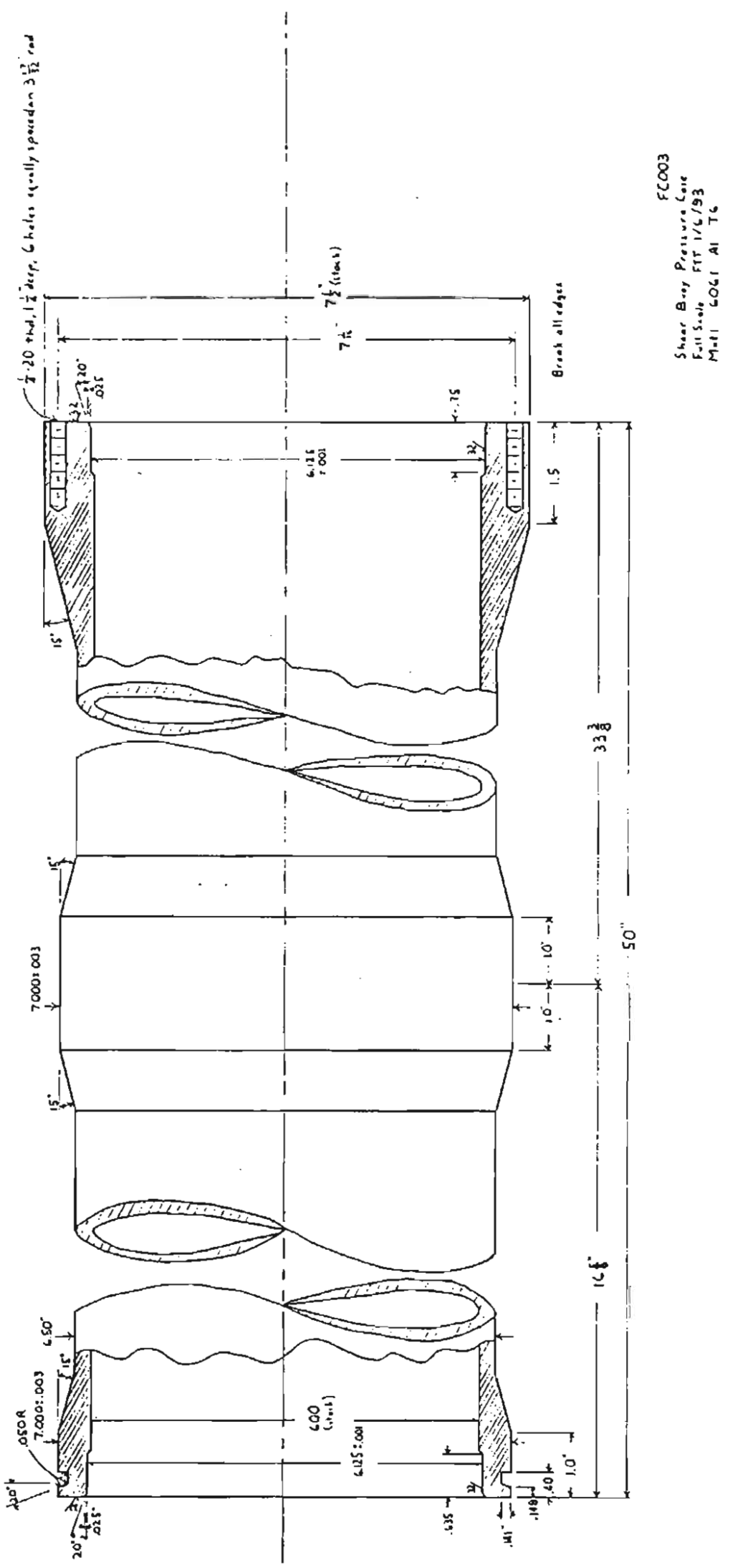

Fig. A-7. Instrument electronics case detail drawing 


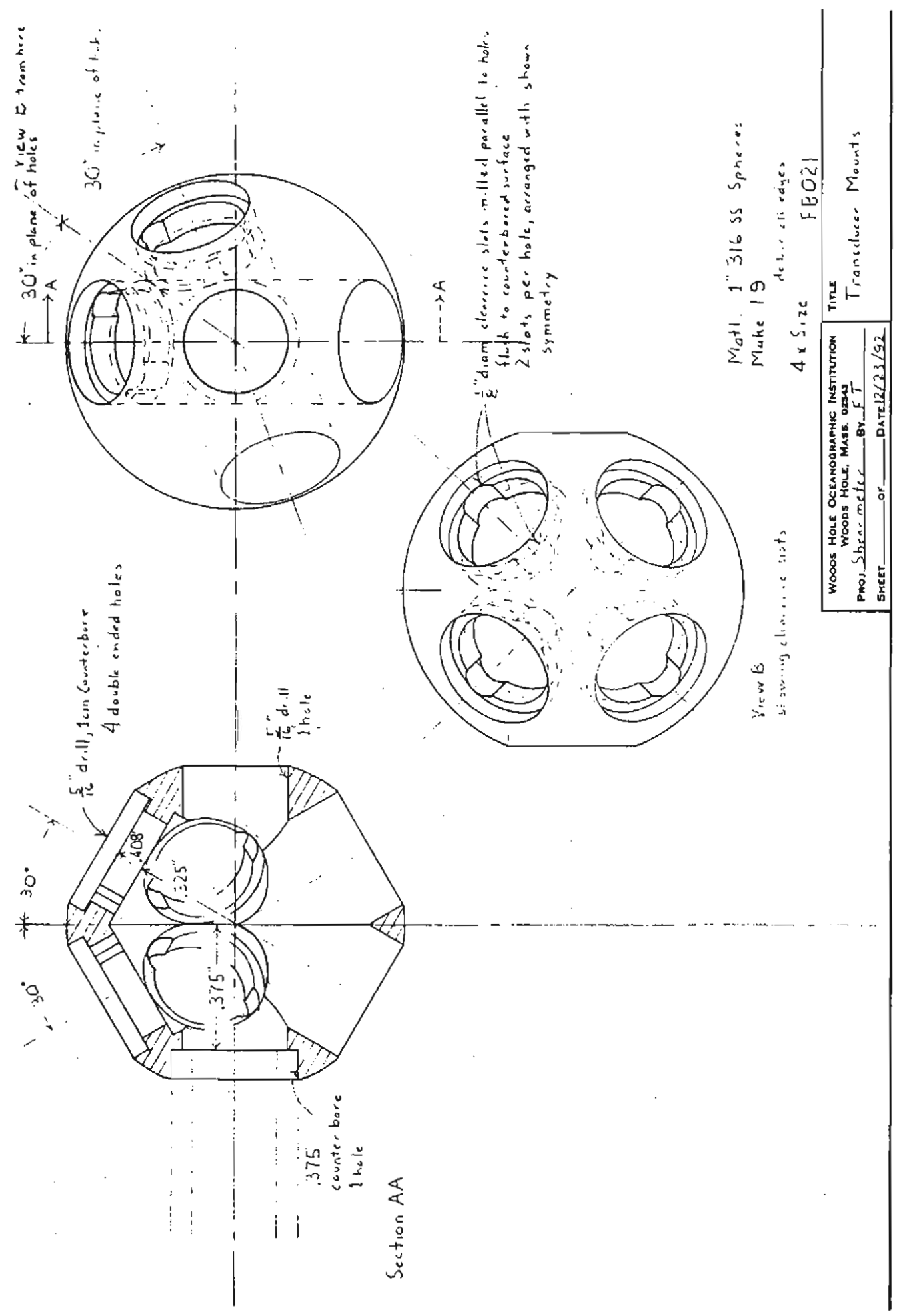

Fig. A-8. Transducer mount detail drawing 


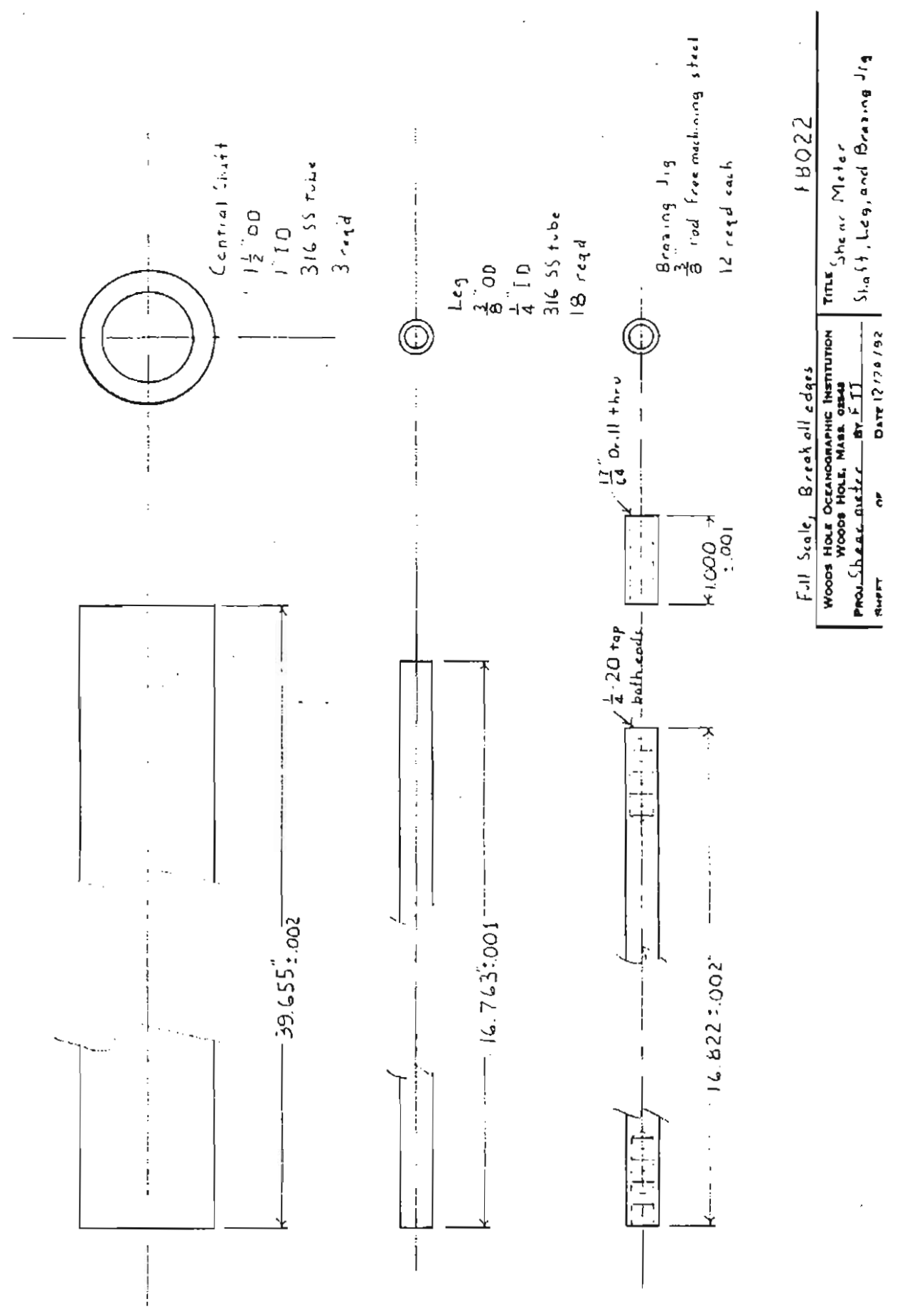

Fig. A-9. Central stalk, pod arms, and braising jig 


\begin{abstract}
APPENDIX B.
Appendix B lists all the processing files for the shear measuring buoy. the processing is done in Matlab and follows the block diagram in Fig. 2-20. The main program is vortcasv. $m$ which calls the various subfunctions and scripts. Remdfc.m is a script file that removes the first and last data records in each $229 \mathrm{~KB}$ disk file. There is a short time gap of about fifteen seconds of unrecorded data when the data logger writes to disk; these disk writes are not coordinated with the start or end of a data record. The unpacking program Bunt.exe can give a corrupted first or last record of a disk file when given incomplete data records. The first and last minute of processed data of each diskfile should be ignored to avoid filter transients. Processing variables are listed and described below in table B-1.
\end{abstract}

\title{
Table B-1 Data Variables
}

\author{
Data variable \\ tim \\ pod1, pod2, pod3, \\ pod4, pod5, pod6 \\ temp \\ imu l \\ imu2 \\ imu3, imu3n \\ imulc \\ imu2c \\ imu $3 \mathrm{c}$ \\ tempc \\ $\operatorname{pod} 1 z, \operatorname{pod} 2 z, \operatorname{pod} 3 z$ \\ $\operatorname{pod} 4 z, \operatorname{pod} 5 z, \operatorname{pod} 6 z$ \\ avel, bvel, cvel, \\ dvel, evel, fvel
}

description

time integers hr:min:s:count

velocity measurements for each circulation path in $\mathrm{A} / \mathrm{D}$ integers

thermistor output in $A / D$ integers

accelerometer output in A/D integers

rate gyro output in $\mathrm{A} / \mathrm{D}$ integers

magnetometer output in $\mathrm{A} / \mathrm{D}$ integers

calibrated accelerometer output in $\mathrm{m} / \mathrm{s}^{2}$

calibrated rate gyro output in $\mathrm{rad} / \mathrm{s}$

calibrated magnetometer output in $\mathrm{mG}$

calibrated thermistor output in $\operatorname{deg} \mathrm{C}$

velocity path zeros in $A / D$ integers

calibrated velocity of each acoustic path of each

circulation path in $\mathrm{cm} / \mathrm{s}$ 
acirc, bcirc, ccirc, dcirc, ecirc, fcirc avort, bvort, cvort dvort, evort, fvort watvelr1, watvelr2 vortb 1, vortb2 watvort l, watvort2

BANGLEF

BANGLEFF

BANGLES

BANGLESF

imu3d

headslow

heading

eulerdot

BANGLE

VORT1, VORT2

watvel1, watvel2

baccel

Buoyvel

Buoyvelf

VEL1, VEL2 circulation over path length for each circulation path in $\mathrm{cm} / \mathrm{s}$

area average vorticity of each circulation path in $1 / \mathrm{s}$ relative sensor velocity in buoy $\mathrm{x}, \mathrm{y}, \mathrm{z}$ coordinates in $\mathrm{cm} / \mathrm{s}$ relative sensor vorticity in buoy $x, y, z$ coordinates in $1 / s$ absolute vorticity in buoy $x, y, z$ coordinates in $1 / s$ integrated rate gyro output in radians highpass filtered rate gyro output in radians slow estimate of buoy Euler angles from accelerometers and magnetometers in radians lowpass filtered slow estimate of buoy Euler angles in radians magnetometer output rotated into computational horizontal frame in $\mathrm{mG}$ unwrapped slow estimate of heading unwrapped estimate of heading Euler angle rates in radians /s buoy Euler angles in radians earth referenced vorticity of each sensor in $1 / \mathrm{s}$ velocity of water in each sensor relative to accelerometer in buoy $x, y, z$ coordinates in $\mathrm{cm} / \mathrm{s}$ buoy acceleration compensated for gravity in $\mathrm{m} / \mathrm{s}^{2}$ buoy velocity in $\mathrm{m} / \mathrm{s}$ in earth coordinates high pass filtered buoy velocity in earth coordinates in $\mathrm{m} / \mathrm{s}$ water velocity in each sensor in earth coordinates in $\mathrm{cm} / \mathrm{s}$ 


\section{Program listing}

$\%$ vortcasv.m is a script file that loads the raw vorticity meter $\%$ data with vortload $\%$ removes change of tattletale diskfile change wild points $\%$ switches imu3,2 and 3 to make up for wiring error $\%$ converts imu data into physical variables

$\%$ converts thermistor data into temperature in deg $\mathrm{C}$ $\%$ clears many of the no longer needed variables

$\%$ last revision 4/13/95

$\%$

vortload

remdfc

imu3n=imu3swtch(imu3);

n2cal4

clear imu 1 imu $1 z$ imu1g imu2 imu2z imu2g imu3 imu3z imu3g dift imu3n tempc $=$ n2t 4 (temp);

clear temp $\mathrm{x}$

svzer

$\mathrm{n} 2 \mathrm{v}$

clear pod 1 pod 2 pod 3 pod $1 z$ pod $2 z$ pod $3 z$ pod 4 pod $4 z$ pod 5 pod $5 z$ pod 6 pod $6 z$

$\%$ Have variables avel acirc avort. imulc imu $2 \mathrm{c}$ imu $3 \mathrm{c}$

$\%$ optional line to clear circulations and use pack command

clear acirc bcirc ccirc dcirc ecirc fcirc

pack

[watvelr1] =veltr45f(avel,bvel,cvel);

[watvelr2]=veltr45f(dvel, evel,fvel);

buoyangb

$\%$ optional line to clear buoyangl intermediate variables

clear BANGLES BANGLEF BANGLEFF

headrate $=[\operatorname{diff}(B A N G L E S F(:, 3)) . / .15 ; 0]+$ filtfilt(Bfh,Afh,imu2c(:,3));

[vortb1,watvort 1]=vortran2(avort, bvort,cvort,[imu2c(:,1:2) headrate]);

[vortb2, watvort2]=vortran2(dvort, evort, fvort, $[$ imu2c $(:, 1: 2)$ headrate]);

$\%$ optional line to clear vel and sensor vort variables

clear avel bvel cvel dvel evel avort bvort cvort dvort evort

clear BANGLESF

VORT $1=b 2 \mathrm{e}$ (watvort $1, \mathrm{BANGLE}$ );

VORT2=b2e(watvort2,BANGLE);

rel2abs 4

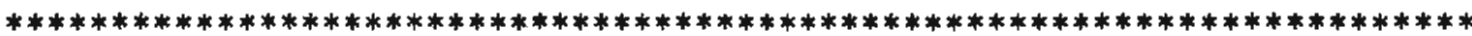

$\%$ VORTLOAD is a script file that loads the mat files from

$\%$ the bunt program, and converts the two's complement numbers

$\%$ from the BASS fluid velocities into regular integers. 
$\%$ This version assumes the vorticity measurements are in pod 1 , $\%$ pod 2 , and pod 3 only.

$\%$

load time

$\operatorname{tim}=x$;

load pod 1

pod1=two 2 reg(x);

load pod2

$\operatorname{pod} 2=$ two $2 \operatorname{reg}(x)$;

load pod3

$\operatorname{pod} 3=\operatorname{two} 2 \operatorname{reg}(x)$;

load pod4

$\operatorname{pod} 4=$ two $2 r e g(x)$;

load pod5

pod5=two2reg(x);

load pod6

pod6=two2reg $(x)$;

load temp

temp $=\mathrm{x}$;

load imu 1

imu l=x;

load imu2

imu2=x;

load imu 3

imu $3=x$;

remdfc

function $\mathrm{y}=\mathrm{two} 2 \mathrm{reg}(\mathrm{x})$

$\%$ function two2reg takes the matlab floating point number

$\% \quad$ from a tattletale BASS current meter 16 bit two's

$\%$ complement number and converts it to signed number.

$\mathrm{I}=$ find $\left(\mathrm{x}>=2^{\wedge} 15\right)$

$\mathrm{y}=\mathrm{x}$;

$y(I)=x(I)-2 \wedge 16$

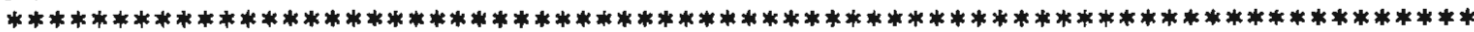

$\%$ REMDFC is a script file that finds the time gaps from the

$\%$ tim,3 and tim, 4 files and removes the corresponding records in

$\%$ all the variables. The file version assumes that all

$\%$ fluid velocities are in pod 1 , pod2, and pod3.

$\%$ This version will not work for sample rates slower than $0.25 \mathrm{~Hz}$.

$\%$ Last revision 6/12/94

$\%$

$\operatorname{difs}=\operatorname{diff(}(\operatorname{tim}(:, 3))$; 


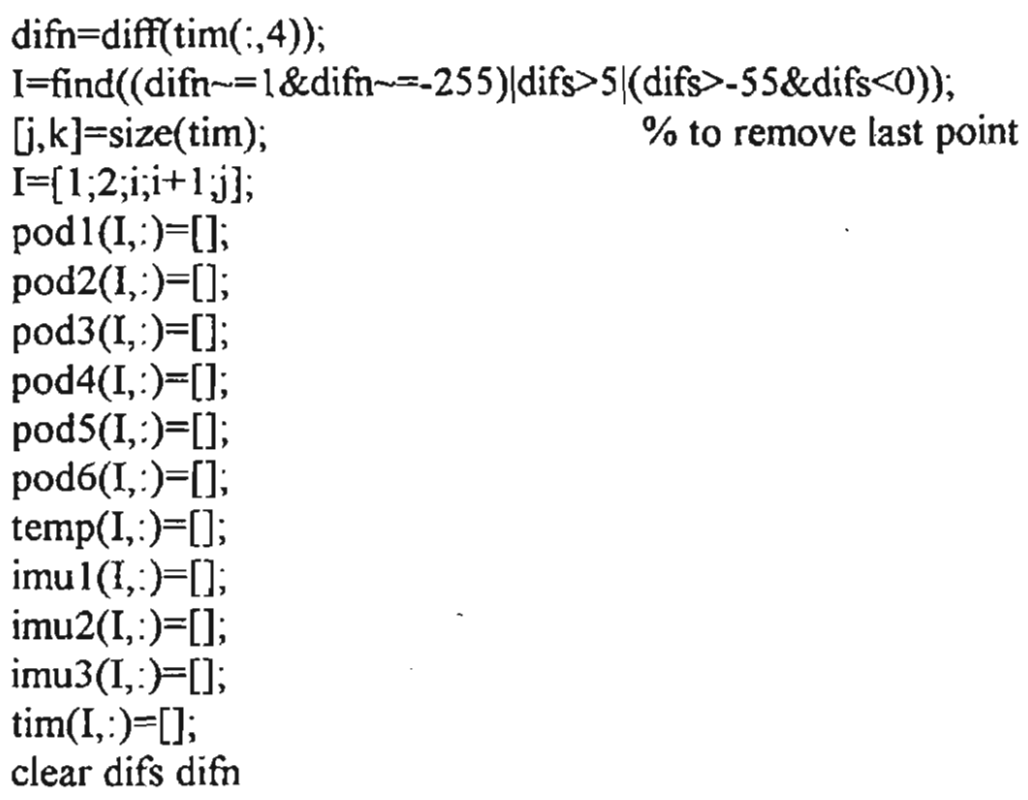

$\% \mathrm{n} 2 \mathrm{cal} . \mathrm{m}$ is a script file to convert vorticity float Bass AVD output to $\%$ physical units using linear calibrations found earlier.

$\%$ imu $1 \mathrm{c}$ in $\mathrm{m} / \mathrm{sec}^{\wedge} 2$

$\% \mathrm{imu} 2 \mathrm{c}$ in rad/sec

$\%$ imu $3 \mathrm{c}$ in $\mathrm{mG}$ and assume imu3,2 and 3 have already been switched

$\%$ establish gain and zero constants,

$\%$ these calibration constants are from 3/8/94

imulg $=[-0.000300530 .000301420 .00030121]$;

imulz=[9.9501 -9.9478 -0.2539];

imu2g $=[2.6848 \mathrm{e}-5$ 2.6965e-5 2.6691e-5];

imu $2 z=\left[\begin{array}{lll}-0.9006 & -0.8951 & -0.8767\end{array}\right]$;

imu $3 g=[0.0190560 .018742-0.017240]$;

imu $3 z=\left[\begin{array}{llll}-643.2 & -581.4 & 533.3\end{array}\right]$;

$\%$ do conversions

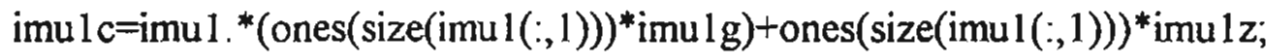


imu2c=imu2. ${ }^{*}\left(\right.$ ones $(\operatorname{size}(i m u 2(:, 1))){ }^{*}$ imu2g) +ones(size(imu2(:, 1)))*imu2z; imu3c=imu3n. ${ }^{*}\left(\right.$ ones(size(imu3n(:, 1))) ${ }^{*}$ imu3g)+ones(size(imu3n(:,1)))*imu3z; $\%$ optional lines to clear up memory space clear imul imu2 imu3 imu3n

function $[\mathrm{t}]=\mathrm{n} 2 \mathrm{t}(\mathrm{n})$

$\%$ function $\mathrm{t}=\mathrm{n} 2 \mathrm{t}$ (bassint)

$\%$ This function computes the temperature in degrees Centigrade

$\%$ from the Bass recorded integer using the Steinhart and Hart

$\%$ equation.

$\% \quad 1 / \mathrm{T}($ in $\operatorname{deg} \mathrm{K})=\mathrm{a}+\mathrm{b}^{*} \ln (\mathrm{r})+\mathrm{c}^{*}(\ln (\mathrm{r}))^{\wedge} 3$

$\%$ Coefficients from the YSI catalog for 44030 thermistor

$a=0.0014051$

$b=0.0002369$;

$\mathrm{c}=1.019 \mathrm{e}-7$

$\%$

$\%$ calculate thermistor resistance from bass recorded A/D number

$\%$ using bridge and amplifier gains from vorticity meter

$\%$

$[\mathrm{i}, \mathrm{j}]=\operatorname{size}(\mathrm{n})$;

$\mathrm{r}=\left(.016032^{*}\left(2^{\wedge} 15^{*}\right.\right.$ ones $\left.(\mathrm{i}, \mathrm{j})-\mathrm{n}\right)+1685^{*}$ ones $\left.(\mathrm{i}, \mathrm{j})\right) . /\left(.387^{*}\right.$ ones $(\mathrm{i}, \mathrm{j})+\ldots$

$5.8314 \mathrm{e}-6^{*}\left(\mathrm{n}-2^{\wedge} 15^{*}\right.$ ones $\left.\left.(\mathrm{i}, \mathrm{j})\right)\right)$;

$\%$

$\%$ compute temperature from thermistor resistance

$\%$

invK=a*ones $(\mathrm{i}, \mathrm{j})+\left(\mathrm{b}^{*}\right.$ ones $\left.(\mathrm{i}, \mathrm{j})\right){ }^{*} \log (\mathrm{r})+(\mathrm{c} *$ ones $\left.(\mathrm{i}, \mathrm{j})) \cdot{ }^{*}((\log (\mathrm{r})))^{\wedge} 3\right)$; $\mathrm{t}=$ ones $(\mathrm{i}, \mathrm{j})$./invK -273.15 ;

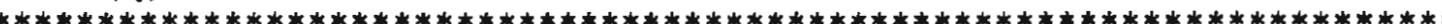

$\%$ svzer.m

$\%$ svzer.m is a script file to load velocity zeros (in signed integers)

$\%$ for the double sensor deployment south of Martha's Vineyard on 5/27/94.

$\%$ Sensor 1 into cards 1,2 and 3 and sensor 2 into cards 4,5, and 6.

$\%$ C, 1 diode fixed.

$\%$ Sensor 1 zeros from svzer462-470 and sensor 2 zeros from svzer333-450.

$\%$ last revision $6 / 14 / 94$

$\%$

podl $z=\left[\begin{array}{llll}5.8 & 15.9 & -6.6 & 65.2\end{array}\right]$;

pod $2 z=\left[\begin{array}{llll}-30.3 & -17.7 & -12.4 & 29.5\end{array}\right]$

$\operatorname{pod} 3 z=\left[\begin{array}{llll}124.3 & -53.3 & -39.5 & -30.7\end{array}\right]$;

$\operatorname{pod} 4 \mathrm{z}=\left[\begin{array}{llll}23.9 & -154.5 & -29.3 & 256.8\end{array}\right]$

pod $5 z=\left[\begin{array}{llll}-33.1 & -191.8 & -109.1 & 214.6\end{array}\right]$

pod $6 z=\left[\begin{array}{llll}28.6 & -173.5 & 34.2 & 265.5\end{array}\right]$

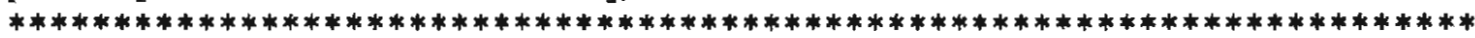


$\%$ n2v.m

$\% \mathrm{n} 2 \mathrm{v} . \mathrm{m}$ is a script file that converts $45 \mathrm{~cm}$ vorticity meter signed

$\%$ integer velocity numbers and calculates corresponding path velocities

$\%$ in $\mathrm{cm} / \mathrm{sec}$, "circulation" in $\mathrm{cm} / \mathrm{sec}$, and vorticity in $\mathrm{sec}^{\wedge}-1$.

$\%$ This file assumes that appropriate zeros(pod $1 z$; pod $2 z$, etc.) have

$\%$ been loaded into matlab.

$\%$ This is the version for Matlab 4.0 and two sensors

$\%$ last revision $6 / 13 / 94$

$\%$

avel $=.0037^{*}(\operatorname{pod} 1-\text { ones }(\operatorname{size}(\operatorname{pod} 1(:, 1))))^{*}$ pod $\left.1 z\right)$;

acirc=avel $(:, 1)+$ avel $(:, 2)+\operatorname{avel}(:, 3)+\operatorname{avel}(:, 4)$;

avort=acirc/47.34;

bvel $=.0037 *\left(\operatorname{pod} 2-\right.$ ones $\left.(\operatorname{siże}(\operatorname{pod} 2(:, 1)))^{*} \operatorname{pod} 2 z\right)$;

bcirc=bvel $(:, 1)+$ bvel $(:, 2)+$ bvel $(:, 3)+$ bvel $(:, 4)$;

bvort $=$ bcirc/47.34;

cvel $\left.=.0037^{*}(\operatorname{pod} 3-\text { ones }(\operatorname{size}(\operatorname{pod} 3(;, 1))))^{*} \operatorname{pod} 3 z\right)$;

ccirc $=\operatorname{cvel}(:, 1)+\operatorname{cvel}(:, 2)+\operatorname{cvel}(:, 3)+\operatorname{cvel}(:, 4)$;

cvort $=$ ccirc/47.34;

dvel $=.0037^{*}(\operatorname{pod} 4-\text { ones }(\operatorname{size}(\operatorname{pod} 4(:, 1))))^{*}$ pod4z $)$;

dcirc $=$ dvel $(:, 1)+d v e l(:, 2)+\operatorname{dvel}(:, 3)+\operatorname{dvel}(:, 4)$;

$\mathrm{dvort}=\mathrm{dcirc} / 47.34$;

evel $\left.=.0037^{*}(\operatorname{pod} 5-\text { ones }(\operatorname{size}(\operatorname{pod} 5(:, 1))))^{*} \operatorname{pod} 5 z\right)$;

ecirc=evel $(:, 1)+\operatorname{evel}(:, 2)+\operatorname{evel}(:, 3)+\operatorname{evel}(:, 4)$;

evort=ecirc/47.34;

fvel $=.0037 *$ (pod6-ones $(\text { size }(\operatorname{pod} 6(:, 1)))^{*}$ pod6z);

fcirc $=$ fvel(:,1)+fvel(:,2)+fvel(:,3)+fvel(:,4);

fvort $=$ fcirc/47.34;

$\%$ optional line to clear up some memory

clear pod 1 pod 2 pod3 pod 4 pod5 pod6

function [watvelr] =veltr45f(avel,bvel,cvel)

$\%$ function [watvelr] $=$ veltr $45 f($ avel,bvel,cvel)

$\%$ This function does a coordinate transformation for measured vorticity

$\%$ meter velocities from the sensor paths to buoy axis. Since there are

$\% 12$ measured velocities, some averaging is done.

$\%$ This version is for the $45 \mathrm{~cm}$ path vorticity meter after the chip was

$\%$ changed, ie, for the Massbay deployment and later.

$\%$ set up constants to save later computation

$\mathrm{s} 302=.25^{*} \operatorname{sqrt}(3) / 2$;

$02 \mathrm{~s} 3=.25 /\left(2^{*} \operatorname{sqrt}(3)\right)$;

os $3=.25 /$ squrt( 3$)$;

$\mathrm{s} 203=.25^{*} \operatorname{sqrt}(2 / 3)$;

$\%$ set up the transformation matrix 


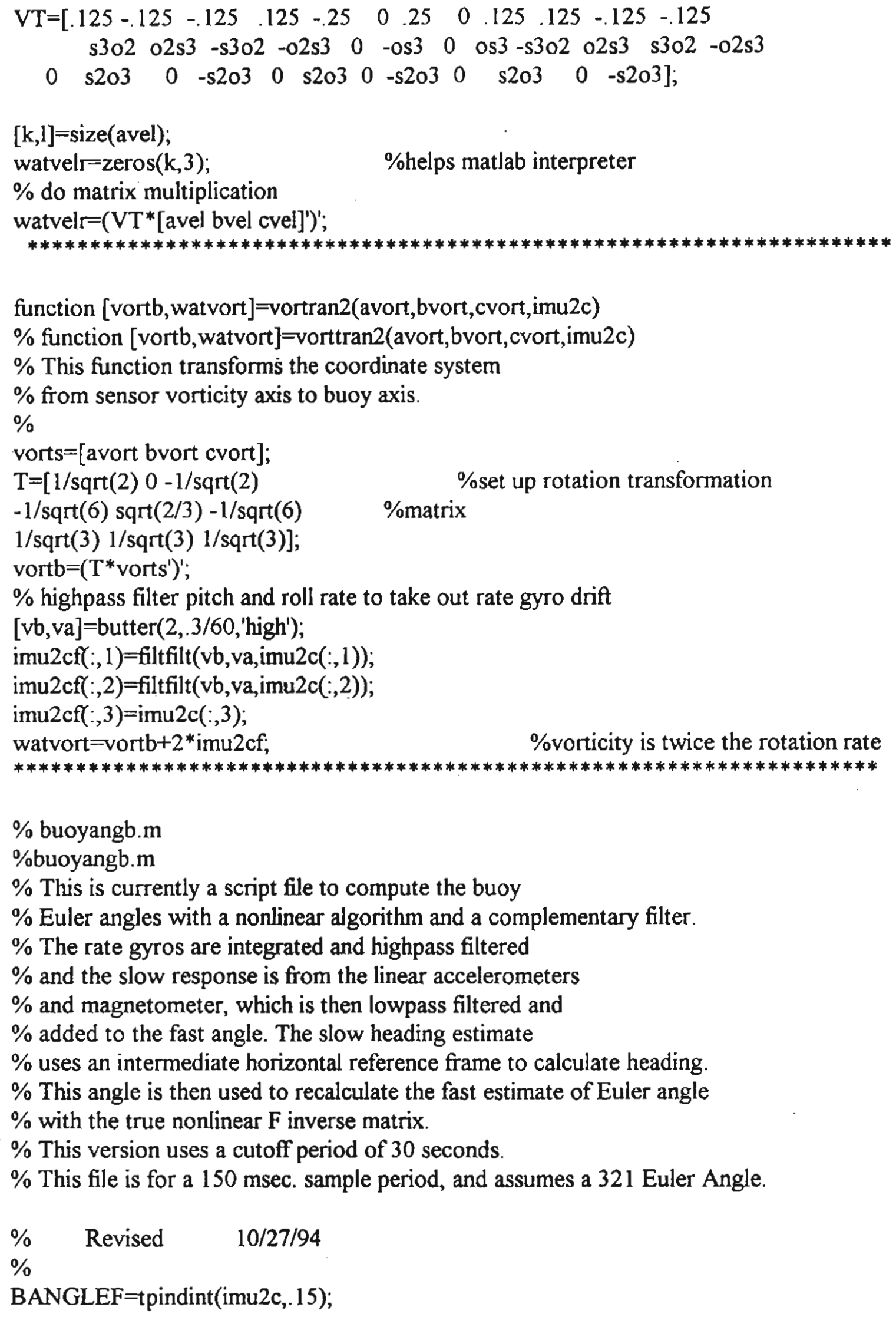




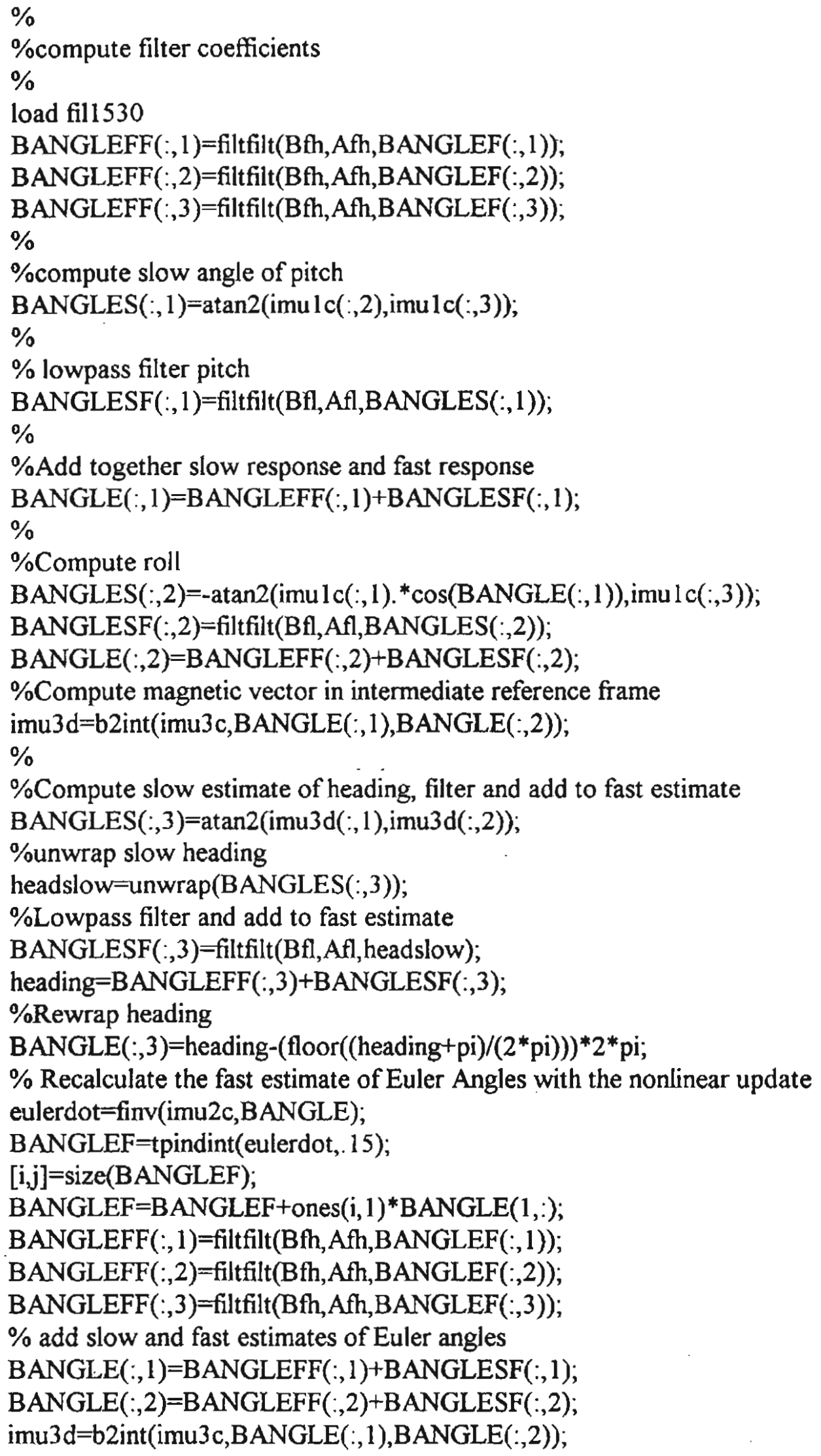


$\%$

\%Recompute slow estimate of heading, filter and add to fast estimate

BANGLES(:,3)=atan2(imu3d(:,1),imu3d(:,2));

\%unwrap slow heading

headslow-unwrap(BANGLES(:,3));

$\%$ Lowpass filter and add to fast estimate

BANGLESF $(; 3)=$ filtfilt(Bfl,Afl, headslow);

heading=BANGLEFF(:,3)+BANGLESF(:,3);

\%Rewrap heading

$\operatorname{BANGLE}(:, 3)=$ heading-(floor $($ heading+pi $) /(2 *$ pi $))) * 2 *$ pi;

$\%$ Optional line to delete intermediate variables

\%clear headslow heading

function [area]=tpindint(func, delx)

$\%$ function [area] $=$ tpindint(func,delx)

$\%$ This function uses the trapezoidal rule to

$\%$ calculate the running area under data.

$\%$ The error should be proportional to the

$\%$ deltax squared times the second derivative

$\%$ of the function. If func is a matrix, [area] will be

$\%$ a matrix with the integration carried for each column

$\%$

$[\mathrm{m}, \mathrm{n}]=\operatorname{size}($ func);

crude=cumsum(func);

$\%$ for vector case

if $\mathrm{m}=\mathrm{l} \mid \mathrm{n}=-1$, area $=($ crude $-.5 *($ func $(1) *$ ones $(\operatorname{size}($ func $))+$ func $)) *$ delx;

$\%$ for matrix case

else

end;

area $=\left(\right.$ crude $-.5^{*}($ ones $(m, 1) *$ func $(1,:)+$ func $\left.)\right) *$ delx;

function [imu3d] $=\mathrm{b} 2 \mathrm{int}(\mathrm{imu} 3 \mathrm{c}$,psi,thet)

$\%$ function imu3d = b2int(imu3c,psi, thet)

$\%$ This function does the coordinate transformation from buoy axis

$\%$ to an intermediate computational reference frame with a vertical

$\% \mathrm{z}$ axis. It is used by the buoyang 2 algorithm to improve it's

$\%$ heading estimate.

$\% \quad$ Revised 6/24/94

$\%$ The following vectors are components of the rotation transformation

$\%$ matrix A21

$\%$

$|123|$ 
$\mathrm{Al}=\cos ($ thet $)$;

$\mathrm{A} 2=\sin (\mathrm{psi}) .{ }^{*} \sin ($ thet $)$

$\mathrm{A} 3=\cos (\mathrm{psi}) *{ }^{*} \sin ($ thet $)$;

A4=zeros(size(psi));

$\mathrm{A} 5=\cos (\mathrm{psi})$;

$\mathrm{A} 6=-\sin (\mathrm{psi})$;

$A 7=-\sin ($ thet $)$;

$\mathrm{A} 8=\sin (\mathrm{psi}) \cdot{ }^{*} \cos ($ thet $)$;

$\mathrm{A} 9=\cos (\mathrm{psi}){ }^{*} \cos ($ thet $)$;

$\%$ size the imu $3 \mathrm{~d}$ matrix to save compiler time

imu3d=zeros(size(imu3c));

imu $3 d(:, 1)=A 1 .{ }^{*}$ imu $3 c(:, 1)+A 2 . *_{i m u} 3 c(:, 2)+A 3 . *^{*} \operatorname{imu} 3 c(:, 3)$;

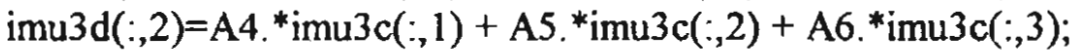

imu3d $(:, 3)=A 7,{ }^{*}$ imu3c(:,1) + A8. ${ }^{*}$ imu $3 c(:, 2)+A 9 .{ }^{*}$ imu3c(:,3);

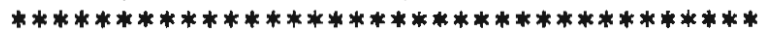

function eulerdot=finv(imu2c,BANGLE)

$\%$ function eulerdot $=$ finv $(i m u 2 c, B A N G L E)$

$\%$ This function multiplies buoy oriented angle rates by

$\%$ the $\mathrm{F}$ inverse matrix for the nonlinear Euler Angle

$\%$ update.

$\% \quad$ revised $\quad 10 / 27 / 94$

$\%$ make vectors for less typing

psi=BANGLE $(:, 1)$;

thet=BANGLE $(:, 2)$;

phi=BANGLE $(:, 3)$;

$\%$ matrix $\mathrm{F}$ inverse $\mid 123$ |

$\% \quad|456|$

$\% \quad|789|$

$\mathrm{fl}=$ ones(size(psi));

$\mathrm{f} 2=\tan ($ thet $) .{ }^{*} \sin (\mathrm{psi})$;

$\mathrm{f} 3=\tan ($ thet $) .{ }^{*} \cos (\mathrm{psi})$;

$\mathrm{f} 4=\mathrm{zeros}(\mathrm{size}(\mathrm{psi}))$;

$\mathrm{f} 5=\cos (\mathrm{psi})$;

$f 6=-\sin (p s i)$;

$\mathrm{f} 7=\mathrm{f} 4$;

$\mathrm{f} 8=\sin (\mathrm{psi}) . / \cos$ (thet);

$9=\cos (p s i) \cdot / \cos ($ thet $)$; 


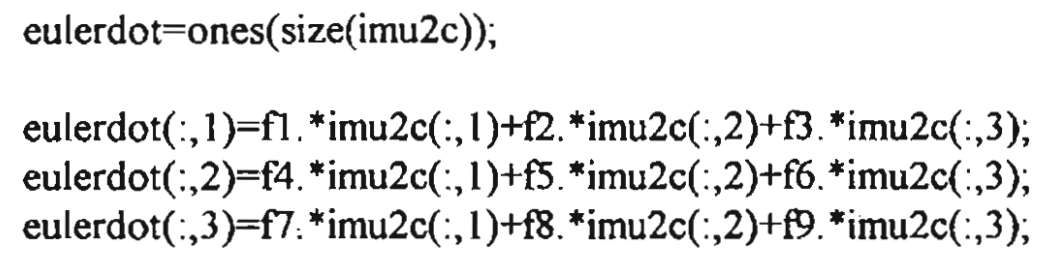

function $[\mathrm{EVect}]=\mathrm{b} 2 \mathrm{e}(\mathrm{bvect}, \mathrm{BANGLE})$

\%function EVect=b2e(bvect,BANGLE)

$6 / 7 / 94$

$\%$ This function does the coordinate transformation from buoy

$\%$ axis to earth axis

$\%$

$\%$ make vectors for less typing

psi=BANGLE $(:, 1)$;

thet=BANGLE $(:, 2)$;

phi=BANGLE $(:, 3)$;

$\%$ The following vectors are components of the rotation transformation

$\%$ matrix A

$\left|\begin{array}{lll}1 & 2 & 3\end{array}\right|$

$\%$

$|456|$

$\%$

$|789|$

$\mathrm{Al}=\cos ($ thet $) \cdot{ }^{*} \cos (\mathrm{ph})$

$\mathrm{A} 2=\sin (\mathrm{psi}) .{ }^{*} \sin ($ thet $){ }^{*} \cos (\mathrm{phi})-\cos (\mathrm{psi}) .{ }^{*} \sin (\mathrm{phi}) ;$

$A 3=\cos (p s i) .{ }^{*} \sin ($ thet $) .{ }^{*} \cos (p h i)+\sin (p s i) .{ }^{*} \sin (p h i) ;$

$\mathrm{A} 4=\cos ($ thet $){ }^{*} \sin (\mathrm{phi})$;

A5 $=\sin (p s i) .{ }^{*} \sin ($ thet $) .{ }^{*} \sin (p h i)+\cos (p s i) .{ }^{*} \cos (p h i) ;$

A6 $=\cos (p s i) .{ }^{*} \sin ($ thet $) .{ }^{*} \sin (p h i)-\sin (p s i) .{ }^{*} \cos (p h i)$;

$A 7=-\sin ($ thet $)$;

$\mathrm{A} 8=\cos ($ thet $){ }^{*} \sin (\mathrm{psi})$;

$\mathrm{A} 9=\cos ($ thet $) .{ }^{*} \cos (\mathrm{psi})$;

\%size the EVect matrix to save the compiler time

EVect=ones(size(bvect));

$\operatorname{EVect}(:, 1)=A 1 .{ }^{*}$ bvect $(:, 1)+A 2 .{ }^{*}$ bvect $(:, 2)+A 3$. ${ }^{\text {bvect }}(:, 3)$;

$\operatorname{EVect}(:, 2)=A 4$. ${ }^{*}$ vect $(:, 1)+A 5$. bvect $(:, 2)+A 6 .{ }^{*}$ bvect $(:, 3)$;

EVect $(:, 3)=A 7 .{ }^{*}$ bvect $(:, 1)+A 8 .{ }^{*}$ bvect $(:, 2)+A 9$ *bvect $(:, 3)$;

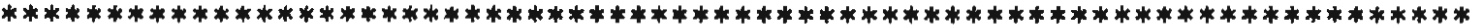

$\%$ rel2abs $4 . m$

$\%$ rel2abs $4 . m$

$\%$ This is a script file that computes water velocity in earth

$\%$ coordinates. This algorithm adds the omega cross $r$ term in

$\%$ the buoy reference and computes and adds the buoy velocity

$\%$ in earth coordinates.

$\%$ This version is for $.15 \mathrm{sec}$ sampling and buoy velocity is

$\%$ highpass filtered in buoy coordinates and in earth coords

$\%$ revised $2 / 27 / 95$ 
$\mathrm{rl}=157 ; \quad \%$ radius in centimeters

$\mathrm{r} 2=318.7$;

$\mathrm{g}=9.8 ; \quad \% \mathrm{~m} / \mathrm{sec}^{\wedge} 2$

$[\mathrm{n}, \mathrm{m}]=\operatorname{size}($ watvelr $\mathrm{l})$;

$\%$ Add in omega cross $r$ term

watvel $1=$ watvelr $1+\left[\right.$ imu $2 c(:, 2)^{*} \mathrm{r} 1-\mathrm{imu} 2 \mathrm{c}(:, 1)^{*} \mathrm{r} 1$ zeros(n, 1)];

watvel $2=$ watvel $r 2+\left[\operatorname{imu} 2 c(:, 2){ }^{*} r 2\right.$-imu $2 c(:, 1) * r 2$ zeros $\left.(n, 1)\right]$;

$\%$ Compensate buoy acceleration for gravity

baccel=imu l c $+\left[\mathrm{g}^{*} \sin (\operatorname{BANGLE}(:, 2))-\mathrm{g} * \sin (\operatorname{BANGLE}(:, 1)){ }^{*} \cos (\operatorname{BANGLE}(:, 2)) \ldots\right.$

-g* $\cos ($ BANGLE $(:, 2)) .{ }^{*} \cos ($ BANGLE $\left.(:, 1))\right]$;

$\%$

$\%$ compute and use highpass filter coefficients

[bfh, afh] $=$ butter $(2,3 / 30$, 'high');

Buoyvel=tpindint(b2e(baccel,BANGLE), 15); \% Velocity in m/sec

$\%$ highpass filter a second time now in earth coords

Buoyvelf $=$ zeros $(\mathrm{n}, \mathrm{m})$;

Buoyvelf(:,1) $=$ filtfilt(bth,afh,Buoyvel(:,1));

Buoyvelf(:,2)=filtfilt(bfh, afh,Buoyvel(:,2));

Buoyvelf $(:, 3)=$ filtfilt $($ bfh, afh,Buoyvel $(:, 3))$;

VELl $=$ b2e(watvel1,BANGLE) $+100 *$ Buoyvelf;

$\%$ VEL in $\mathrm{cm} / \mathrm{sec}$

VEL2=b2e(watvel2,BANGLE)+100*Buoyvelf;

$\%$ watvel in $\mathrm{cm} / \mathrm{sec}$

$\%$ optional line to clear intermediate variables

$\%$ clear watvell watvel 2 baccel Buoyvel Buoyvelf bfh afh 


\begin{abstract}
APPENDIX C.
This appendix catalogs the rotating arm bias measurements made at the David Taylor Model Basin, shows the measured bias in constant flow of the 15-centimeter path vorticity sensor, and shows the measured buoy response functions. In the rotating arm tests, the $45 \mathrm{~cm}$ path vorticity sensor was mounted on an apparatus which moved the sensor in a circular motion keeping all axes constant. All points of the sensor traversed in the same circular motion relative to the tow tank carriage, as the carriage traveled down a large tank of still water. In these tests: the y sensor direction was parallel to the tank length and carriage motion, the $\mathrm{z}$ direction was vertical, and the $\mathrm{x}$ direction was horizontal and across the tank width. The apparatus traversed the sensor in a circular motion who's axis was the $\mathrm{x}$ direction.
\end{abstract}

The measured vorticity means in constant flow (Fig. 3-10) corrected for center strut wake bias, is repeated as Fig. C-1. The standard error shown is the standard deviation of each sample time series divided by the square root of the number of samples of each mean, and is a measure of how much the sample mean is expected to fluctuate. The measured vorticity means with the sensor rotated at ten second, seven second, five second and 3.8 second periods, are shown in Figs. C-2, C-3, C-4, and C-5.

The measured uncorrected biases of the 15-centimeter path vorticity sensor towed through still water are shown in Fig C-6 with the axes labeled.

The measured buoy response functions are plotted in Fig. C-7. The heave, surge, pitch, and pitch rate response functions were calculated from measured cross spectra between a velocity measured at 0.83 meters depth and the buoy response variable. For each cross spectrum, the amplitude, phase, and coherence are plotted. For frequencies where the coherence is below 0.7 the amplitude and phase may not be meaningful. The heave response function is with respect to vertical velocity at 0.83 meters depth. The North surge response is with respect to north velocity. The east surge response was the same, as one would expect and is not shown. The pitch and pitch rate response functions 
are with respect to horizontal velocity in the pitch plane. The roll and roll rate response functions are the same respectively, as one would expect from symmetry.

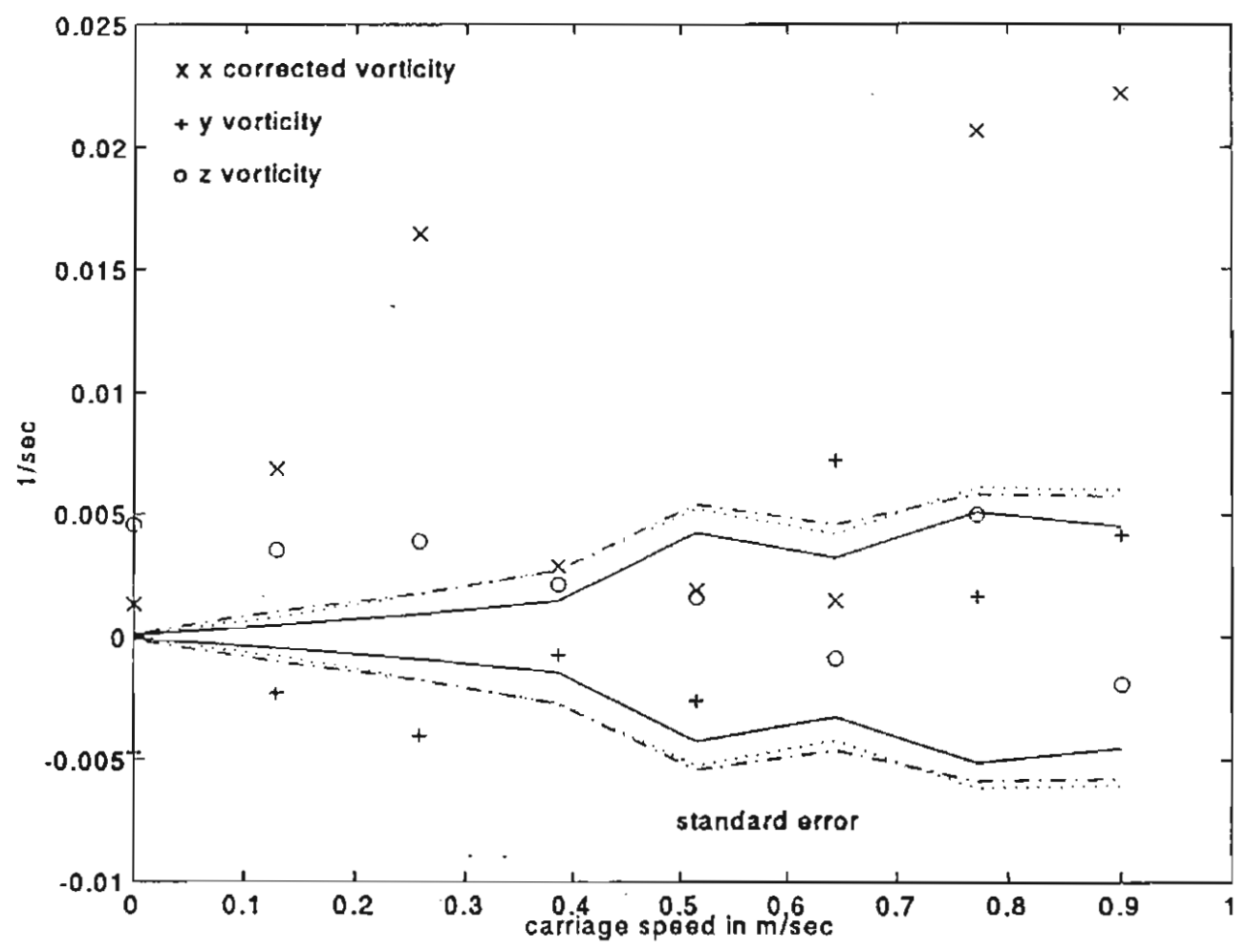

Fig. C-1. Vorticity means in constant flow using the center stalk bias correction described in the text. 


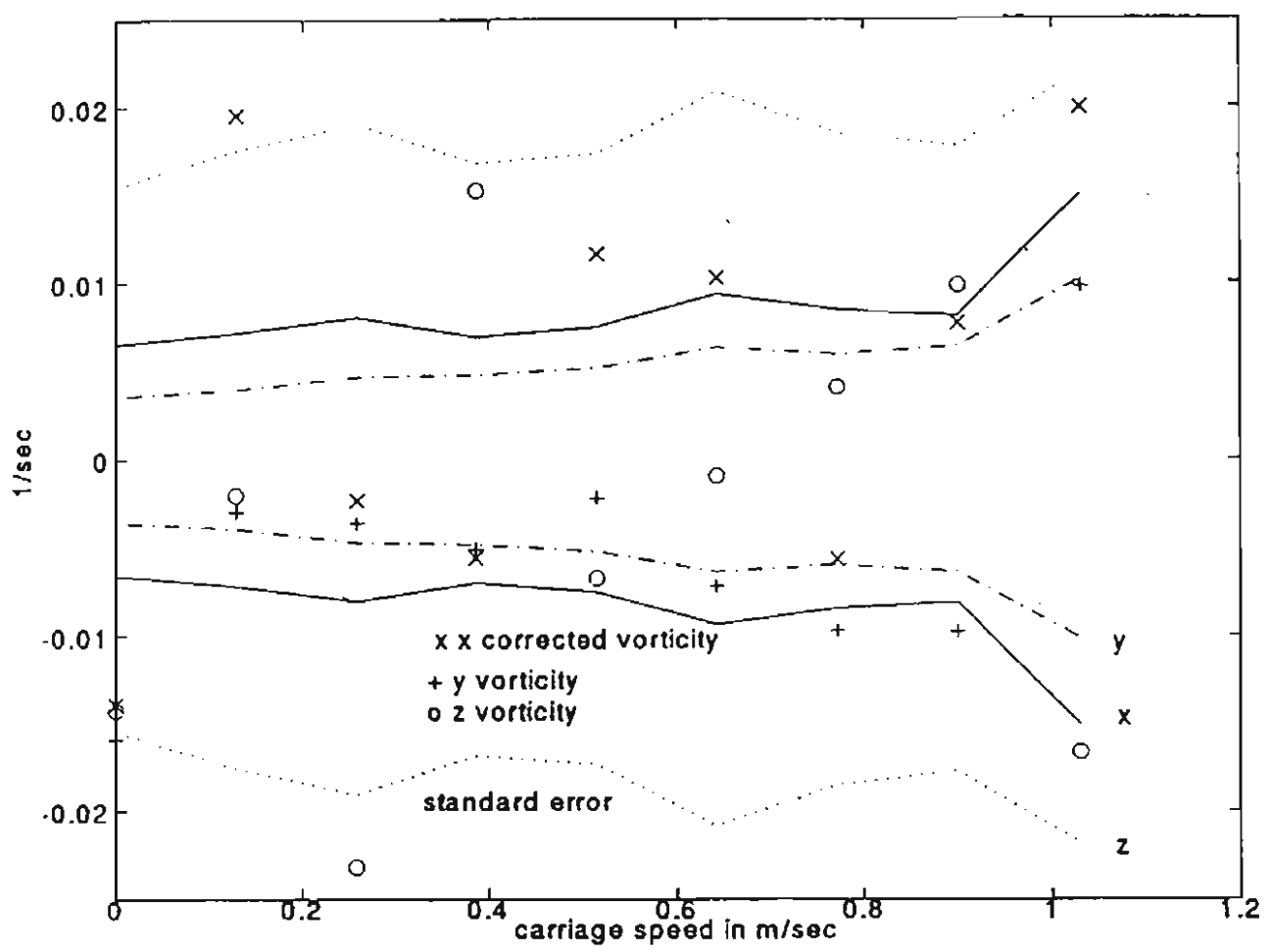

Fig. C-2. Vorticity means with the sensor rotated at a ten second period. The $x-$ axis vorticity is corrected for the center stalk wake bias as described in the text.

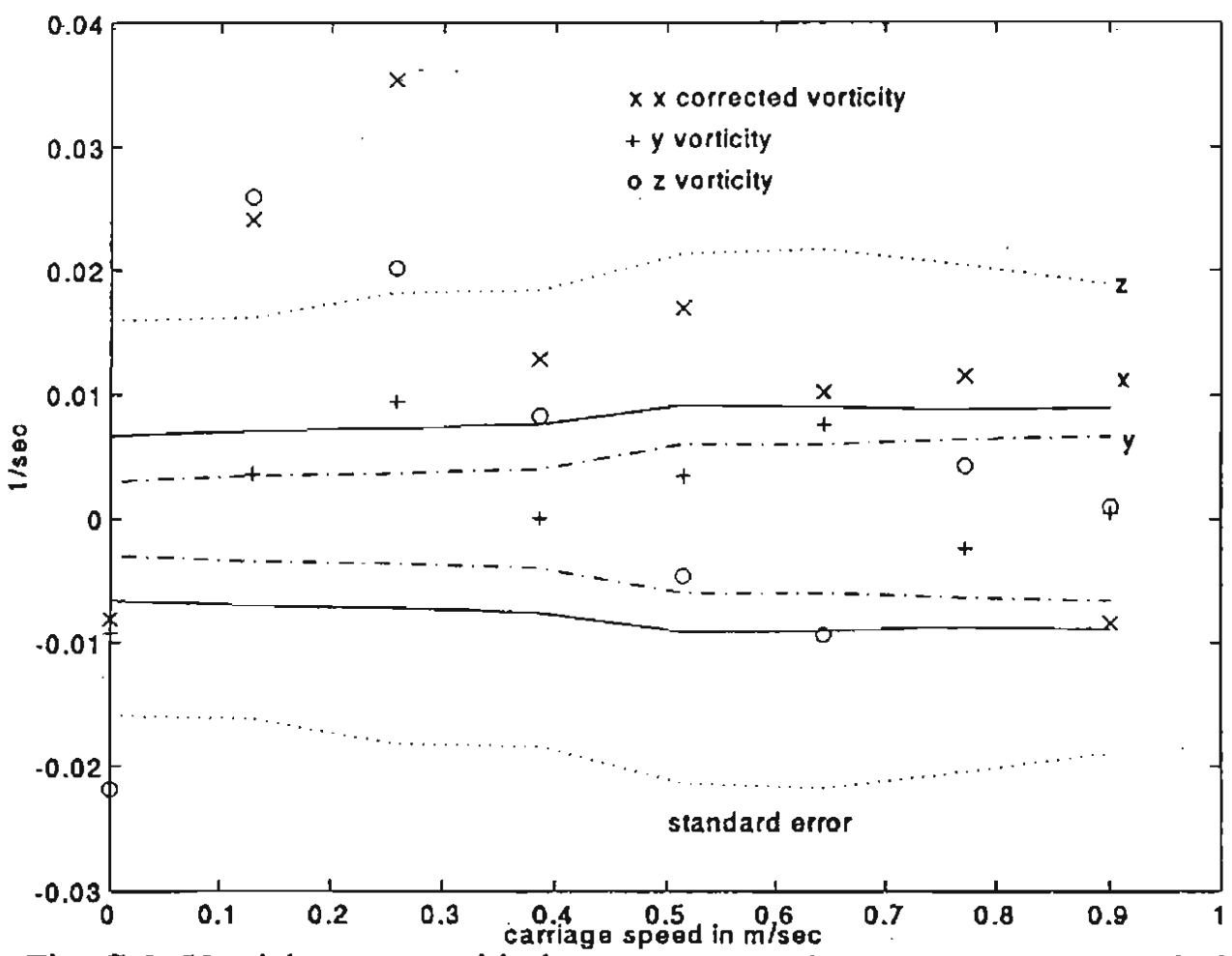

Fig. C-3. Vorticity means with the sensor rotated at a seven second period. The $x-$ axis vorticity is corrected for the center stalk wake bias as described in the text. This figure is a repeat of Fig. 3-15. 


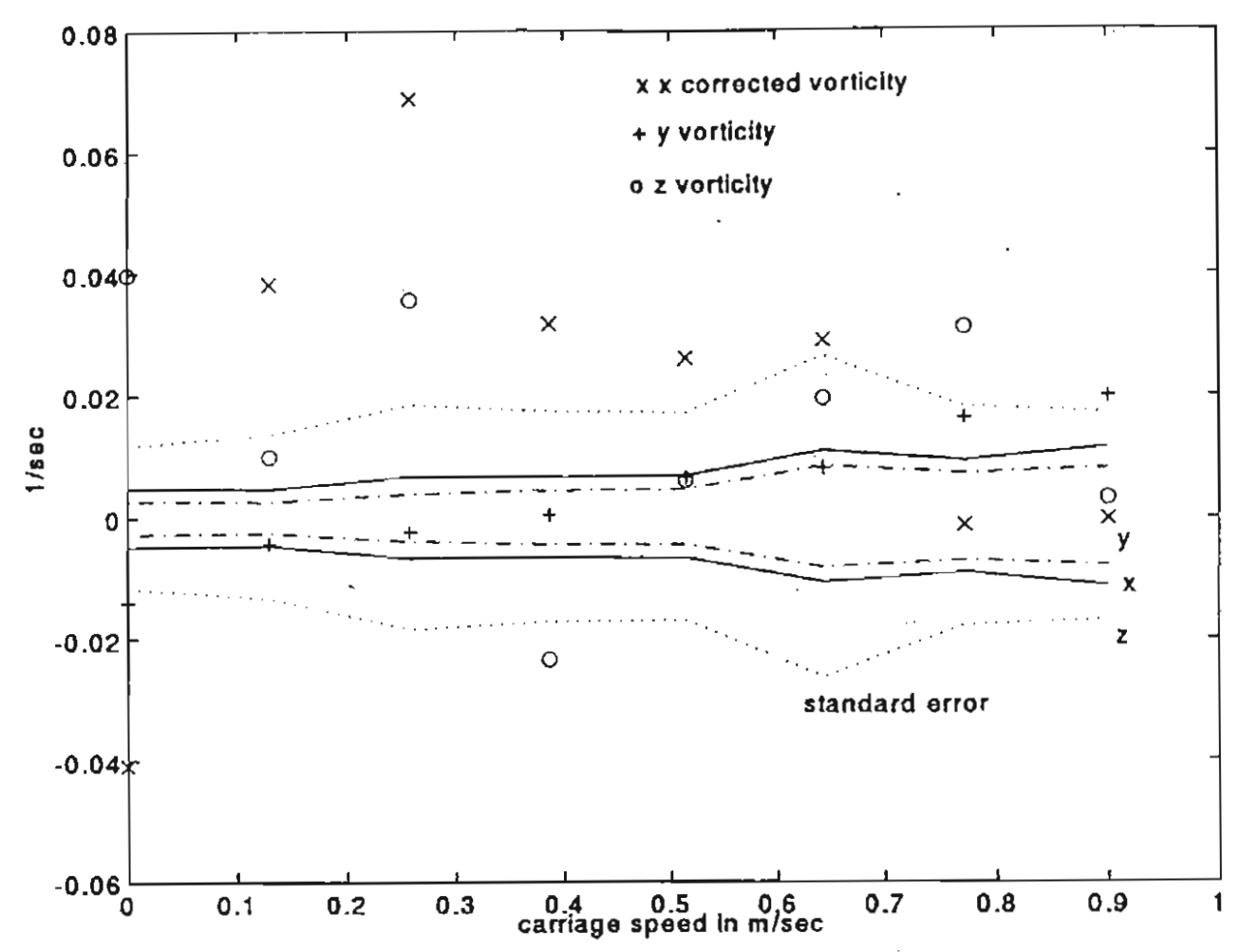

Fig. C-4. Vorticity means with the sensor rotated at a five second period. The $x-$ axis vorticity is corrected for the center stalk wake bias as described in the text.

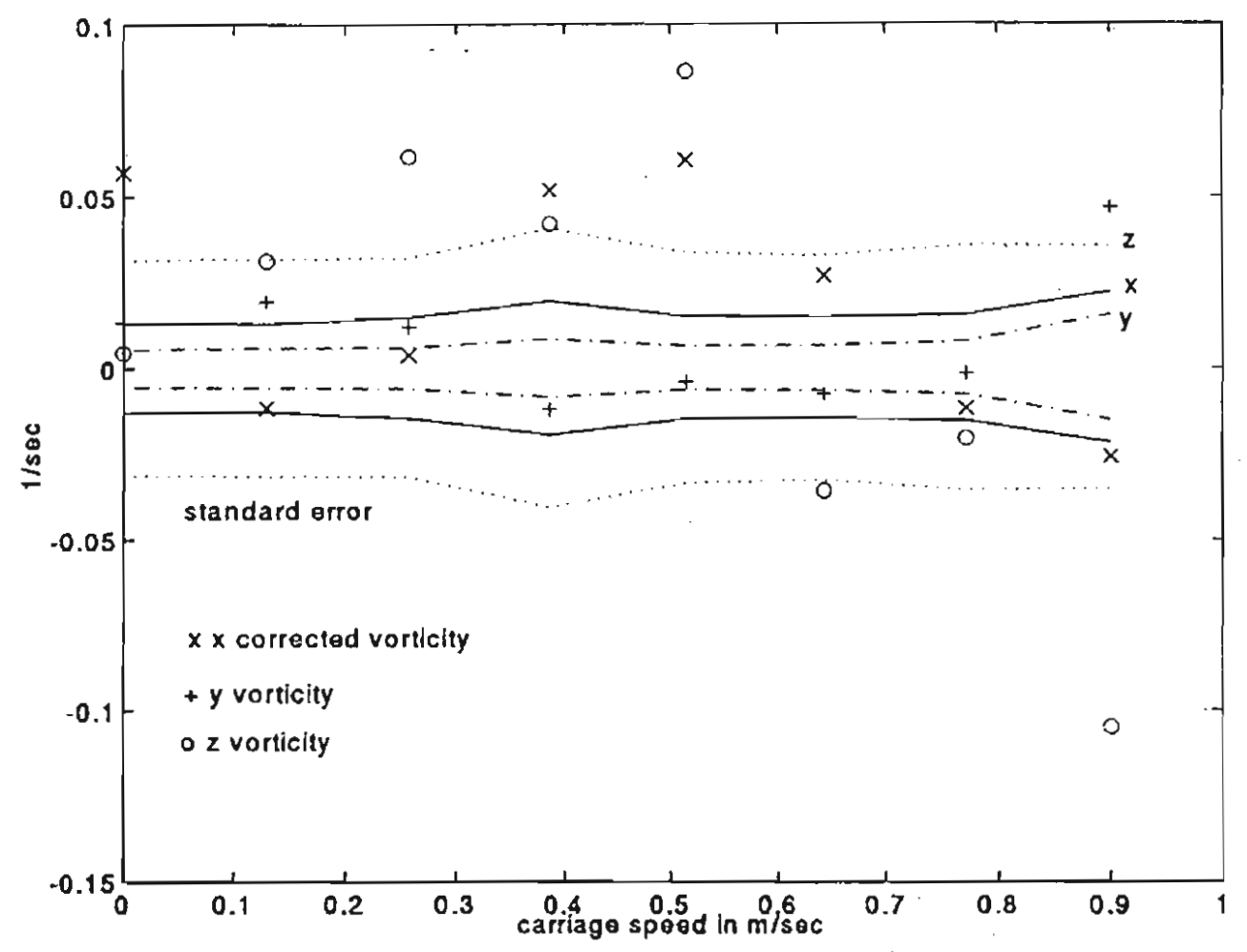

Fig. C-5. Vorticity means with the sensor rotated at a 3.8 second period. The $x-$ axis vorticity is corrected for the center stalk wake bias as described in the text. 


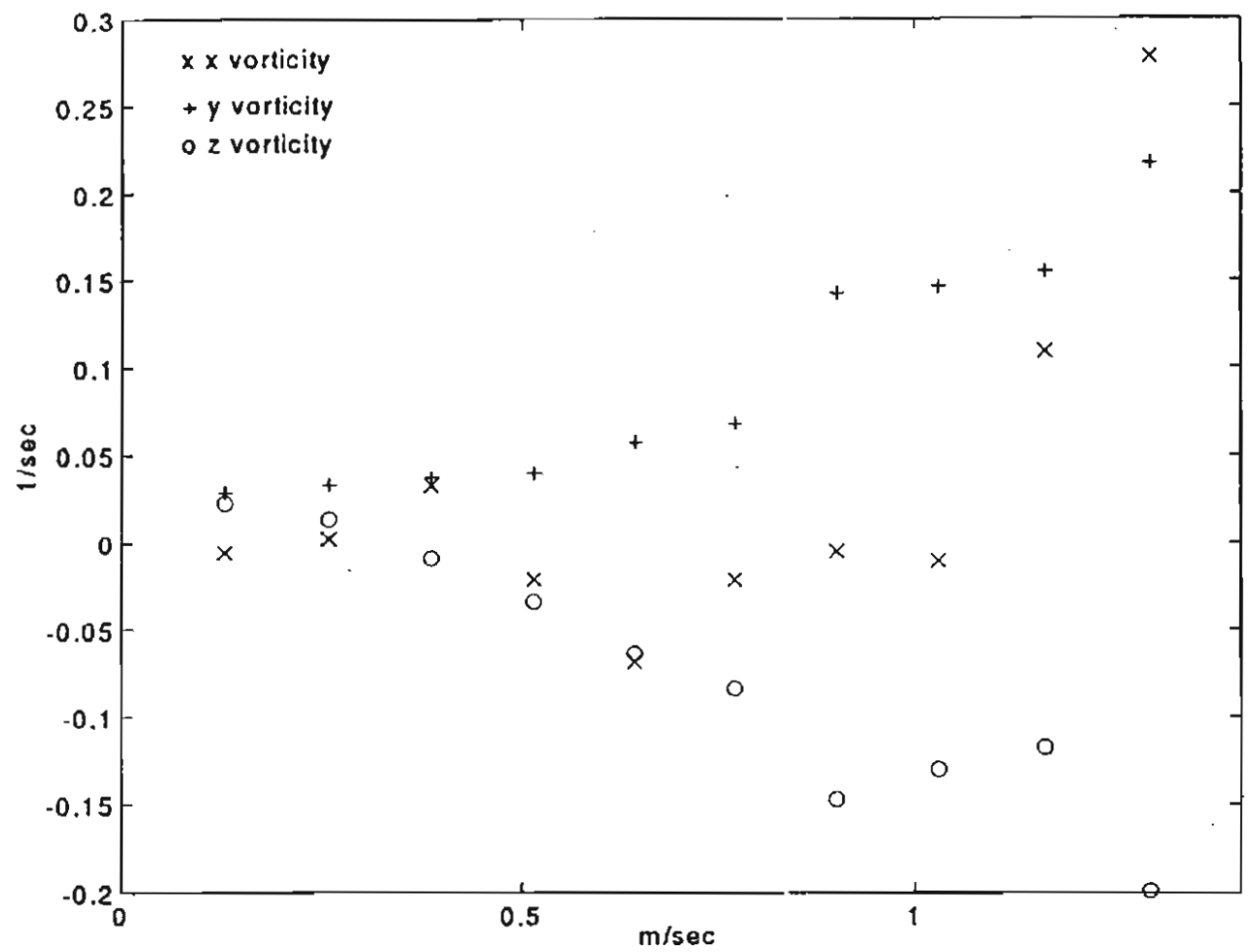

Fig. C-6 Measured biases of $15-\mathrm{cm}$ path vorticity sensor towed through still water. 

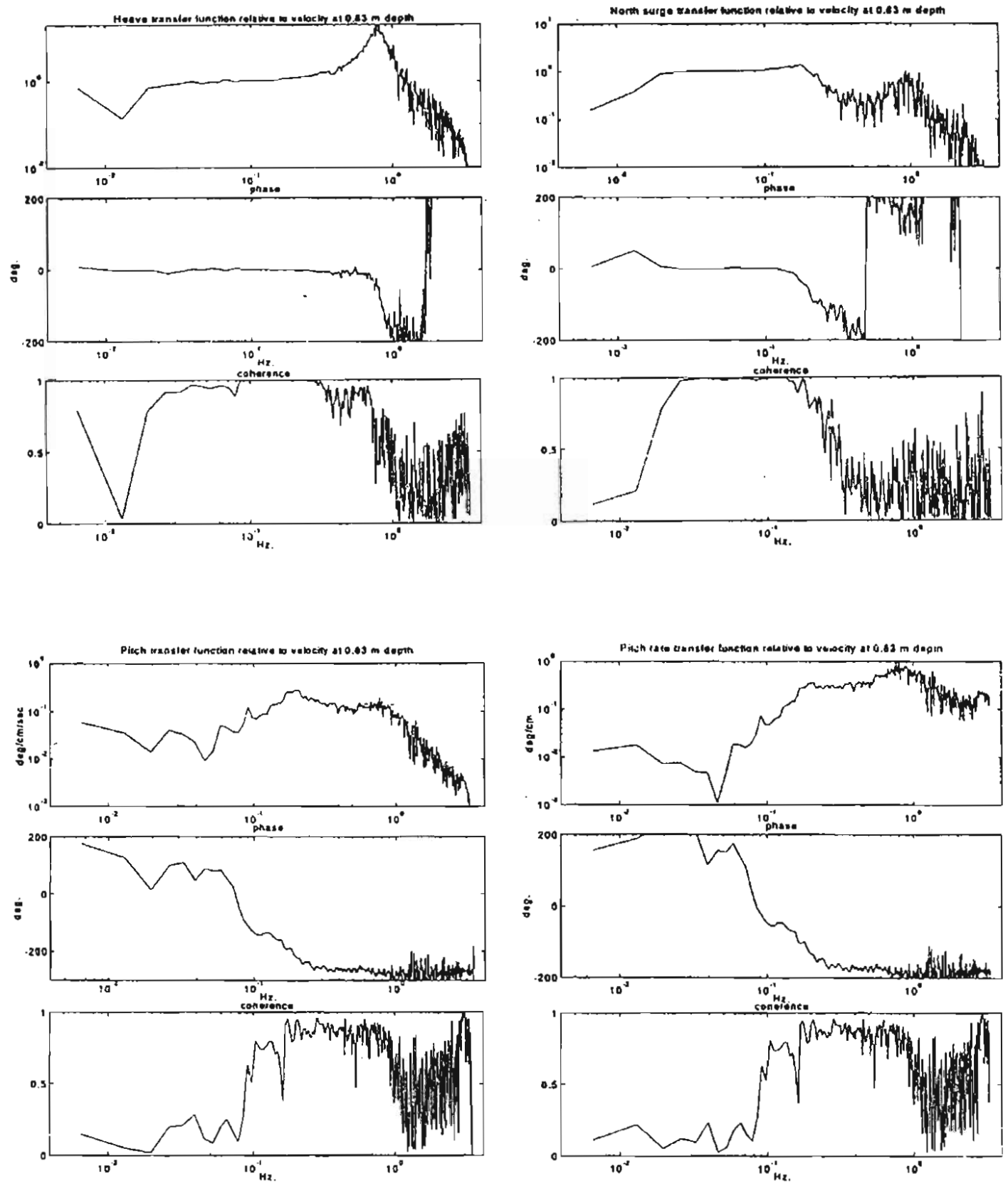

Fig. C-7. Buoy response functions for heave, surge, pitch, and pitch rate relative to velocity at 0.83 meters depth. 


\section{APPENDIX D.}

This appendix rotates the data from the upper-boundary-layer shear deployment into down-wind and cross-wind components, and compares these to two models of boundary-layer behavior. The wind coordinate measurements are compared to two models of boundary layer behavior, the unstratified, turbulent, wall layer and the model by Santala (Santala, 1991). The unstratified-wall-layer shear is given by (D-1) and assumes

$$
\frac{\partial u}{\partial z}=\frac{u}{k z}
$$

no stratification. In this equation, $u$ is the downwind drift velocity, $u_{\bullet}$ is the friction velocity which is the square root of the windstress over the water density, and $K$ is von Karman's constant usually assumed to be 0.4 .

The second model that the data were compared to was developed by Santala to describe ocean-upper-boundary-layer behavior measured in the winter off the northern California coast. In this section, data was rotated into the wind coordinates shown in Fig. $\mathrm{D}-\mathrm{I}$ with $\mathrm{z}$ positive upwards. Recall that the direction of vorticity is defined to be perpendicular to the velocities and directions over which the velocities vary, that the vorticity is derived from. Defining the cross-wind direction for shear opposite from the

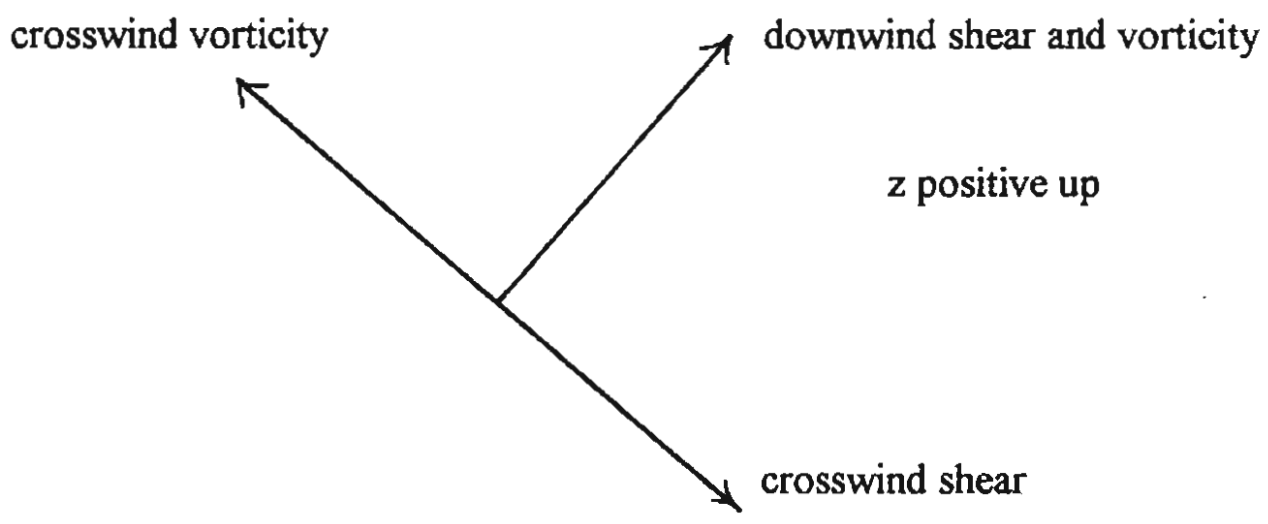

Fig. D-1. Windstress oriented coordinate system used in this appendix. 
cross-wind direction for vorticity at first seems odd, but with this convention positive vorticity corresponds with positive shear. In this coordinate system, the Santala model is given by table D-1. In this table $v_{c}$ is crosswind drift current and $v_{d}$ is the downwind drift

Crosswind shear $\quad \frac{\partial v_{c}}{\partial z}=\frac{1.41 u}{z} \quad$ for $\quad \frac{g z}{u_{0}^{2}}>0.2 * 10^{5}$

Downwind shear

$$
\frac{\partial v_{d}}{\partial z}=\left\{\begin{array}{cc}
0 & 0<\frac{g z}{u_{*}^{2}}<1.2 * 10^{5} \\
\frac{u_{*}}{\kappa z * 1.64 * 10^{5}}\left(\frac{g z}{u_{*}^{2}}-1.24 * 10^{5}\right) & \text { if } 1.24 * 10^{5}<\frac{g z}{u_{*}^{2}}<2.88 * 10^{5} \\
\frac{u_{*}}{k z} & 2.88 * 10^{5}<\frac{g z}{u_{*}^{2}}
\end{array}\right.
$$

Table D-1. Santala upper-boundary-layer shear model in coordinates defined by Fig. D-1

current. This model predicts large crosswind shear and a zero downwind shear layer over a log layer with a transition layer in between. As windstress increases, the inter-layer transitions deepen, and at some depths, the shear can be reduced due to much more effective mixing while the shear stress increases. In this model, Santala does not mention a development time.

The measurements for this comparison were from the upper-boundary-layer deployment described in the first half of the applications section. The shear measuring buoy was deployed in Buzzards Bay, Massachusetts during a period of increasing windstress. Windstress was measured with a sonic anemometer and calculated with the inertial dissipation method (Fairall and Larson, 1986). No evidence of Langmuir cells was seen during the deployment. As the windstress increased, the Santala model transition 
depths deepened, Fig. D-2. When the shallower transition depth sinks below a sensor, the model predicts zero downwind shear.

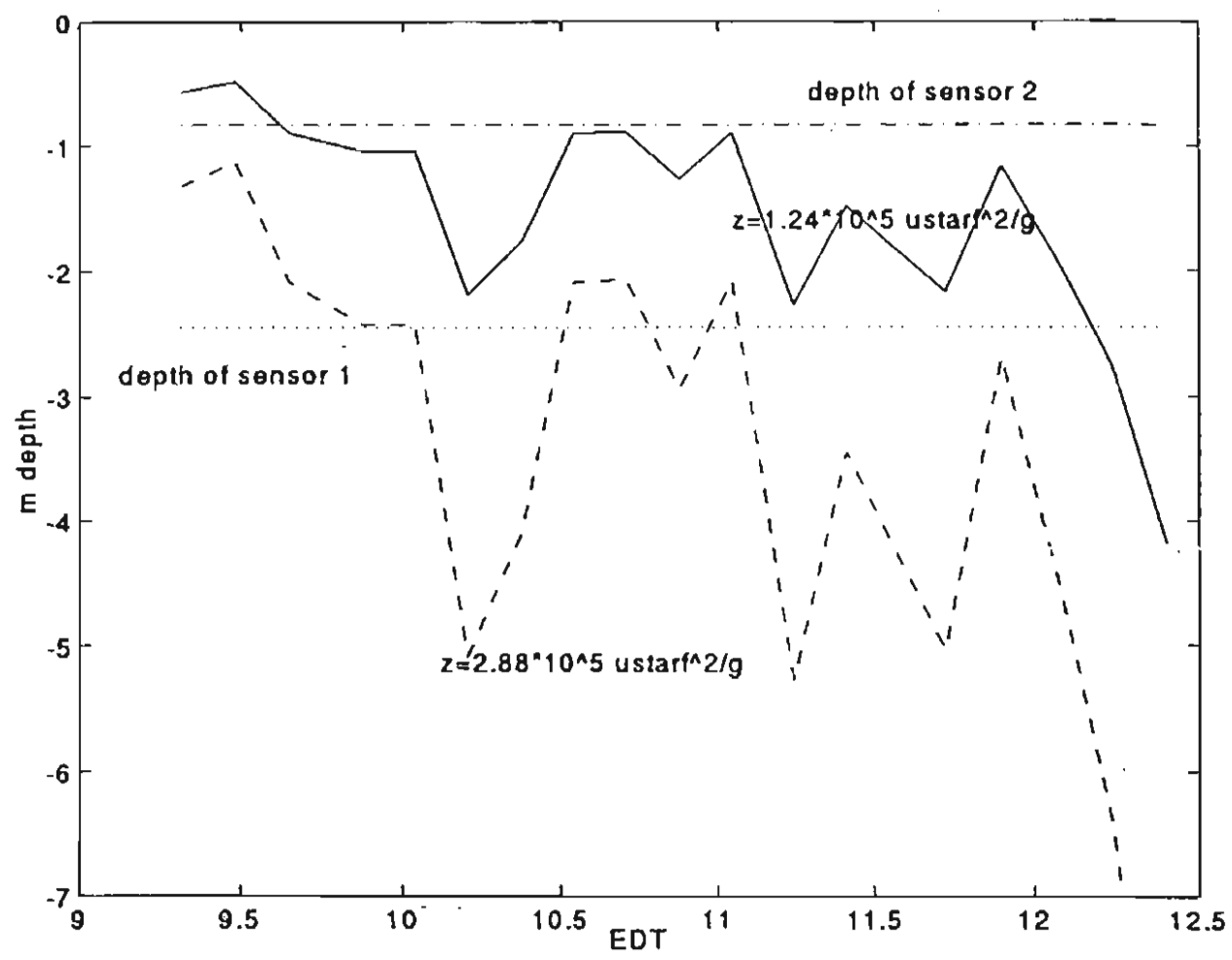

Fig. D-2. Santala model layer transition depths predicted from the measured windstress during the Buzzards Bay deployment

The buoy data were rotated into directions downwind and crosswind, and the vorticity predicted shear were compared to an unstratified wall layer and the Santala model. The downwind shear from vorticity in the $0.83 \mathrm{~m}$ depth sensor is shown plotted with both the Santala model and wall layer model in Fig. D-3. In the first half of the deployment, the shear follows closely the wall model. But, as the windstress continued to increase, the shear diverged from the model and decreased as the windstress increased. With enough time, the measured shear could have approached the Santala model.

The downwind shear from vorticity in the $2.45 \mathrm{~m}$ depth sensor is shown plotted with both models in Fig. D-4. The measured vorticity was much larger than either the 


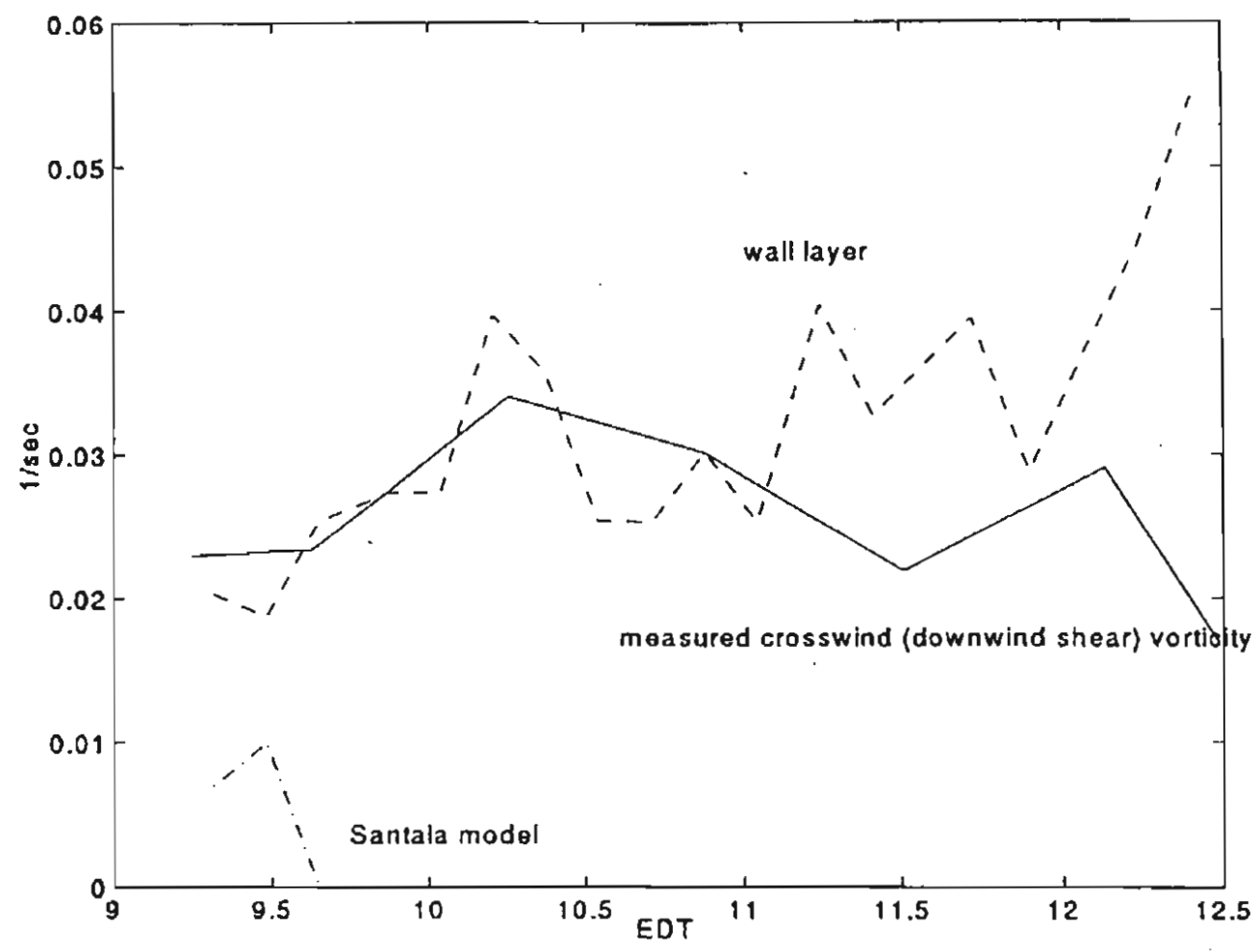

Fig. D-3. Downwind shear from vorticity and windstress $0.83 \mathrm{~m}$ depth

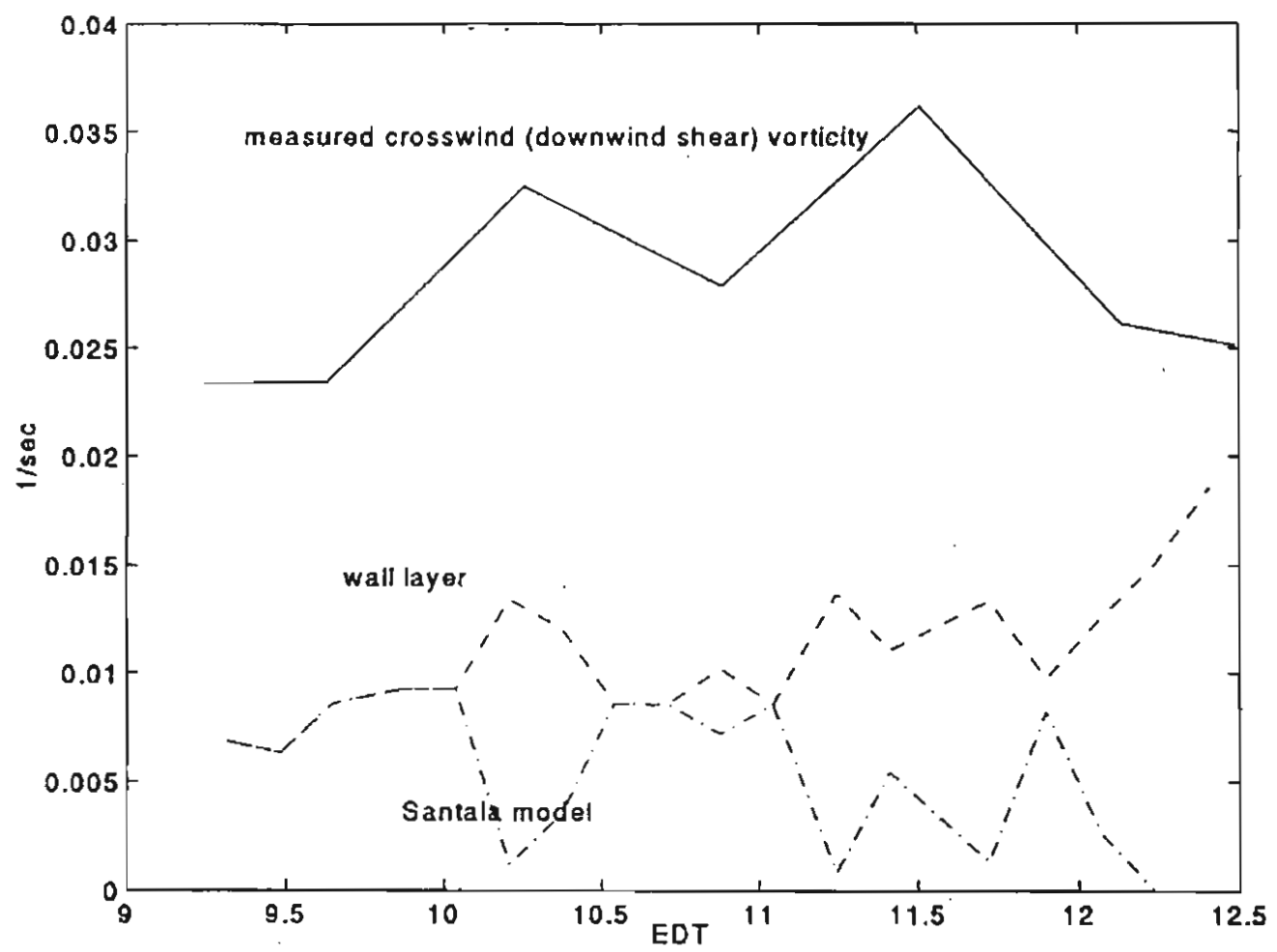

Fig. D-4. Downwind shear from vorticity and windstress, $2.45 \mathrm{~m}$ depth 
wall model or Santala model. The measured stratification over the lower sensor was larger than the stratification between sensors, Fig. D-5. The top thermistor failed leaving only thermistors at the bottom of the shallow measurement volume, and at the top and

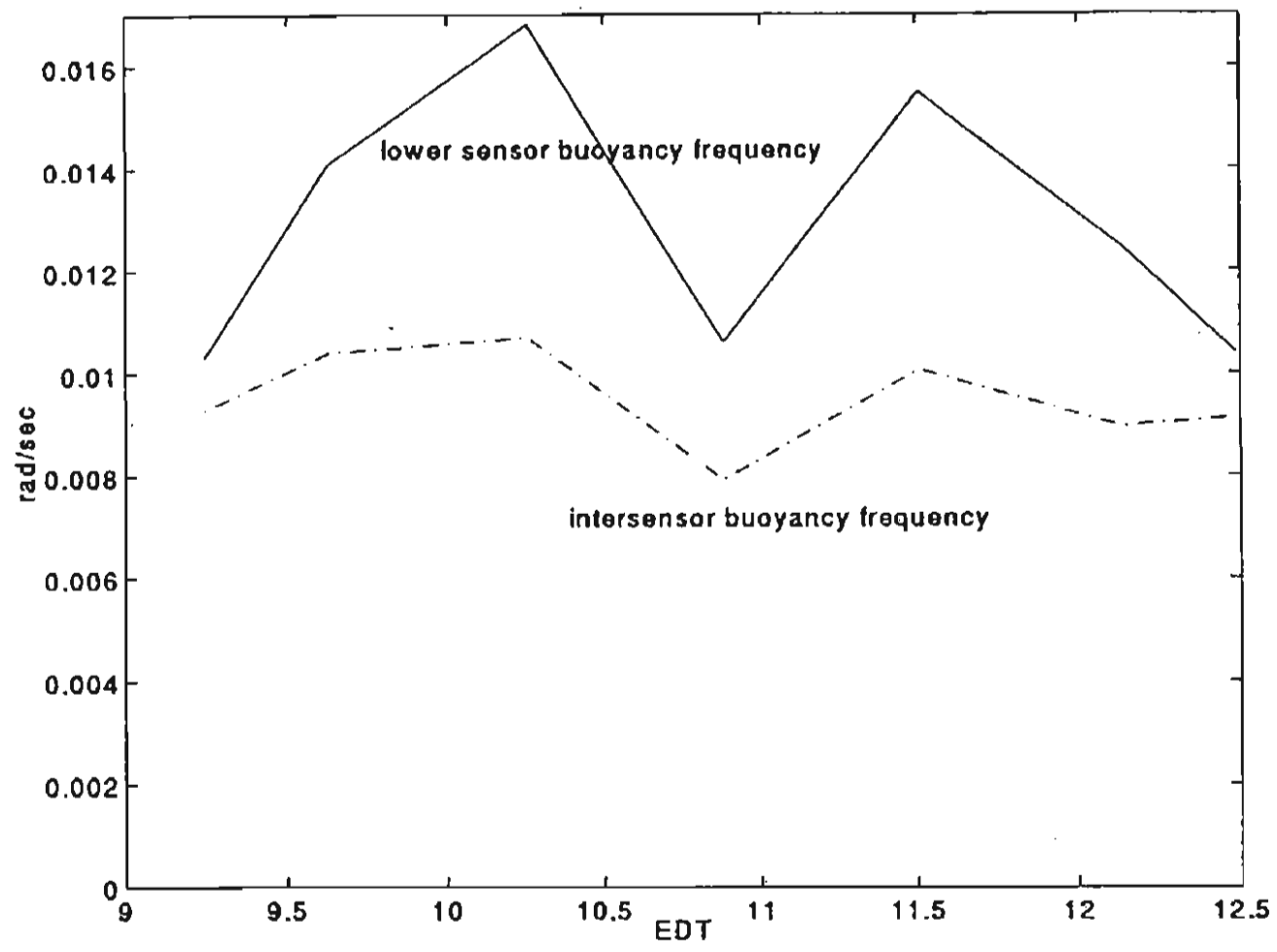

Fig. D-5. Stratification in lower sensor and between sensors

bottom of the deeper measurement volume. The stratification over the shallow sensor was assumed to be the same as the intersensor stratification for the Richardson number plot in the applications section. This larger stratification can explain the less effective vertical turbulent mixing implied by the larger measured shear.

Crosswind shears from measured vorticity and Santala model shear are plotted for both sensors in Fig. D-6. The measured crosswind shear in both sensors was in the same direction as predicted by Santala. The measured crosswind shear in the shallow sensor starts at about zero and does not approach the Santala model until near the end of the deployment when the windstress was strong. The measured crosswind shear over the lower sensor was larger than the Santala model, which can again be explained by the 
larger stratification over the lower sensor. The wall layer model predicts no crosswind shear.

In most of the measurements, the wall layer model, when compensated for stratification, was close to the measured results for low windstress. As the windstress grew, the measured results started to approach the Santala model. The shears measured during this deployment may have been influenced by the shape of Buzzards Bay as the

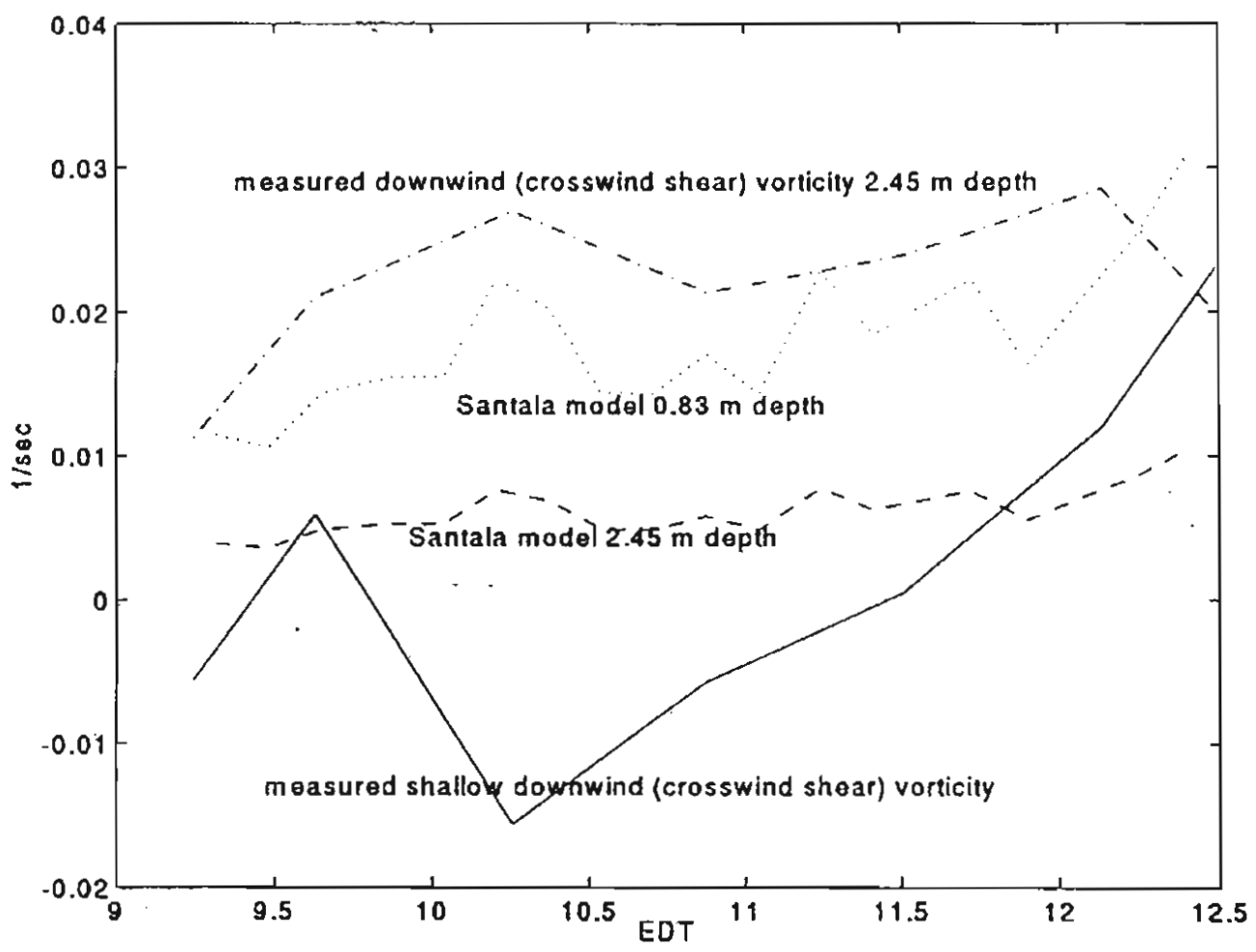

Fig. D-6. Crosswind shear from vorticity and windstress for both sensors.

wave directional spectrum was. The shear measuring buoy is a useful tool to measure shear in the upper five meters of the ocean, and with further and longer deployments should help ocean scientists to better understand this important part of the ocean. 


\section{References}

Agrawal, Y.C., E.A. Terray, M.A. Donelan, P.A. Hwang, A.J. Williams III, W.M. Drennan, K.K. Kahma, and S.A. Kitaigorodskii (1992) Enhanced dissipation of kinetic energy beneath surface waves. Nature, Vol. 359, pp. 219-220.

Axford, D.N. (1968) On the accuracy of wind measurements using an inertial platform in an aircraft, and an example of a measurement of the vertical mesostructure of the atmosphere, J. Appl. Meteor., 7, pp. 645-666.

Barbi, C., D.P. Favier, C.A. Maresca, and D.P.Telionis (1986) Vortex shedding and lockin of a circular cylinder in oscillatory flow. Journal of Fluid Mechanics. vol. 170, pp. $527-544$.

Bearman, P.W. (1984) Vortex shedding from oscillating bluff bodies. Ann. Rev. Fluid Mech. 16, pp. 195-222.

Bell, T.H. Jr. (1975) Topographically generated internal waves in the open ocean. Journal of Geophysical Res. Vol. 80, No. 3, pp. 320-327.

Blevins, R. D. (1977) Flow-Induced Vibration. Van Nostrand Reinhold Co. 363p.

Broecker, H.Ch. and W. Siems (1984) The Role of Bubbles for Gas Transfer from Water to Air at Higher Windspeeds. Experiments in the Wind-wave Facility in Hamburg. in Gas Transfer at Water Surfaces, W. Brutsaert and G.H. Jirka eds. D. Reidel Publishing Co. pp. 229-236.

Brown, R.G. (1983) Random Signal Analysis and Kalman Filtering, John Wiley and Sons. $347 \mathrm{p}$.

Brumley, B.H. (1984) Turbulence Measurements Near the Free Surface in Stirred Grid Experiments. in Gas Transfer at Water Surfaces, W. Brutsaert and G. H. Jirka eds. D. Reidel Publishing Co. pp. 83-92.

Brumley, B.H. and G.H. Jirka (1988) Air-water transfer of slightly soluble bases: turbulence, interfacial processes and conceptual models. PCH PhysicoChemical Hydrodynamics, Vol. 10, No. 3, pp. 295-319.

Cairns, J.L. (1975) Internal wave measurements from a midwater float. Journal of Geophysical Res. vol. 80, no. 3, pp. 299-306.

Caims, J.L., and G.O. Williams (1976) Internal wave observations from a midwater float, 2. Journal of Geophysical Res. vol. 81, no. 12, pp. 1943-1950.

Catfort, J., (1978) Application of Strapdown Inertial Systems With Particular Reference to 
Underwater Vehicles. in NATO Advisory Group for Aerospace Research \& Development (AGARD) Lecture Series No. 95: Strapdown Inertial Systems, Technical

Editing and Reproduction, Ltd., London, pp. 5-1-17.

Cavaleri, L. and S. Zechetto (1987) Reynolds stresses under wind waves. Journal of Geophysical Research, 92, pp. 3894-3904.

Cheung, T.K. and R.L. Street (1988) The turbulent layer in the water at an air-water interface, Journal of Fluid Mechanics, 194, pp. 133-151.

Cheung, T.K. and R.L. Street (1988) Wave-following measurements in the water beneath an air-water interface. Journal of Geophysical Research, vol. 93, pp. 14,089-14,097.

Collar, P.G., R.M. Carson and G. Griffiths (1983) Measurement of near-surface current from a moored wave-slope follower. Deep-sea Research, 30, pp. 63-75.

Craik, A.D.D. (1982) Wave-induced longitudinal-vortex instability in shear flows. J. Fluid Mech. vol. 125, pp. 37-52.

Crandall, S.H., D.C. Karnopp, E.F. Kurtz Jr, and D.C. Pridmore-Brown (1968) Dynamics of Mechanical and Electromechanical Systems, McGraw-Hill. 466p.

Crandall, S.H., S. Vigander, and P.A. March (1975) Destructive Vibration of Trashracks Due to Fluid-Structure Interaction. Journal of Engineering for Industry, pp. 1359-1365.

Crawford, T.L., R.T. McMillen, and R.J. Dobosy (1990) Development of a "Generic" mobile flux platform with demonstration on a small airplane. NOAA Tech.Mem ERL ARL-184.

Csanady, G.T. (1984) The free surface turbulent shear layer. Journal of Physical Oceanography 14, pp. 402-411.

Csanady, G.T. (1985) Air-sea momentum transfer by means of short-crested wavelets. Journal of Physical Oceanography 15, pp. 1486-1501.

D'Asaro, E.A. (1985) Upper ocean temperature structure, inertial currents, and Richardson numbers observed during strong meteorological forcing. Journal of Physical Oceanography vol 15, pp. 943-962.

DeDominicis, T.S. and J.F. McEachern (1990) Drag and strum characteristics of non-round, strum-suppressing cable. IEEE CH2858-9/90/0000-0363 pp363-368.

Desaubies, Y. and W.K. Smith (1982) Statistics of Richardson Number and Instability in Oceanic Internal Waves. Journal of Physical Oceanography, vol. 12, pp. 1245-1259. 
Donelan, M.A. (1978) Whitecaps and momentum transfer. in: Turbulent Fluxes Through the Sea Surface, Wave Dynamics and Prediction, A. Favre and K Hasselman, editors, Plenum Press, pp 273-287.

Dowling, A.P. and J.E. Ffowcs Williams (1983) Sound and Sources of Sound. Ellis Horwood Ltd. West Sussex, England, $321 \mathrm{p}$.

Duda, T.F. and C.S. Cox (1987) Vorticity measurement in a region of coastal ocean eddies by observation of near-inertial oscillations. Geophysical Res. Lett. vol. 14, no.8, pp.793-796.

Dunn, C.V.R. (1984) A miniature urethane molded acoustic transducer. WHOI technical report WHOI-84-30 39p.

Edson, J.B., C.W. Fairall, P.G. Mestayer, and S.E. Larsen (1991) A Study of the Inertial-Dissipation Method for Computing Air-Sea Fluxes. Journal of Geophysical Res., vol. 96, No. C6, pp. 10,689-10,711.

Fairall, C.W. and S.E. Larsen (1986) Inertial-Dissipation Methods and Turbulent Fluxes at the Air-Ocean Interface. Boundary-Layer Meteorology, 34, pp. 287-301.

Faltinsen, O.M. (1990) Sea Loads on Ships and Offshore Structures. Cambridge University Press, 328p.

Favre, A. and K. Hasselmann (1977) Turbulent Fluxes through the sea surface, wave dynamics, and prediction. Plenum Press, 677p.

Fujitani, T. (1985) Method of Turbulent Flux Measurement on a Ship by Using a Stable Platform System. Papers in Meteorology and Geophysics, vol. 36, No. 3, pp. 157-170.

Gargett, A.E.c P.J. Hendricks, T.B. Sanford, T.R. Osborn and A.J. Williams III (1981) A composite spectrum of vertical shear in the upper ocean. Journal of Physical Oceanography, vl 1, No. 9, pp 1258-1271.

Gargett,A.E. and G. Holloway (1984) Dissipation and diffusion by internal wave breaking. Journal of Marine Research, 42, pp. 15-27.

Gargett, A.E. (1989) Ocean Turbulence. Ann. Rev. Fluid Mech. pp. 419-451.

Gargett, A.E. (1990) Do We Really Know How to Scale the Turbulent Kinetic Energy Dissipation Rate $\epsilon$ Due to Breaking of Oceanic Internal Waves? Journal of Geophysical Research, vol. 95, No.C9, pp. 15,971-15,974.

Garrett, C. and W. Munk (1979) Internal waves in the ocean. Ann. Rev. Fluid Mech. 11, pp. 339-69. 
Geernaert, G.L. (1988) Measurements of the angle between the wind vector and wind stress vector in the surface layer over the north sea. Journal of Geophysical Research, vol. 93, pp. 8215-8220.

Geyer, W.R. (1989) Field Calibration of Mixed-Layer Drifters. Journal of Atmospheric and Oceanic Technology, vol. 6, No. 2, pp. 333-342.

Geyer, W.R. and J.R. Ledwell (1994) Final Report: Massachusetts Bay Dye Study, Massachusetts Water Resources Authority, 39p.

Goldstein, H. (1959) Classical Mechanics, Addison-Wesley Publishing Co., Reading, Ma. $399 \mathrm{p}$.

Goodman, L.E. and A.R. Robinson, (1958) Effects of finite rotations on gyroscope devices, Journal of Applied Mechanics, pp. 210-213.

Gordon, A.L. (1970) Vertical Momentum Flux Accomplished by Langmuir Circulation. Journal of Geophysical Res. vol. 75, No. 21, pp. 4177-4179.

Grant, W.D., A.J. Williams,III, and S.M. Glenn (1984) Bottom stress estimates and their prediction on the northern California continental shelf during CODE-1: The Importance of wave-current interaction. Journal of Physical Oceanography 14. pp. 506-527. comments and reply.

Grant, W.D. and O.S. Madsen (1986) The Continental-Shelf Bottom Boundary Layer. Ann. Rev. Fluid Mech. vol. 18, pp. 265-305.

Gregg, M.C. and T.B Sanford (1988) The dependence of turbulent dissipation on stratification in a diffusively stable thermocline. Journal of Geophysical Research, vol. 93 pp. $12,381-12,392$.

Griffiths, G. (1979) The effect of turbulence on the calibration of electromagnetic current sensors and an approximation of their spatial response. Report No. 68, Institute of Oceanographic Sciences, Wormley, Godalming, Surrey.

Hare, J.E. (1992) Shipboard Eddy-covariance measurements of the turbulent fluxes of heat, moisture, and momentum. MS Thesis Penn State University.

Hare, J.E., J.B. Edson, E.J. Bock, and C.W. Fairall (1992) Progress on Direct Covariance Measurements of Air-Sea Fluxes from Ships and Buoys. Tenth Symposium on Turbulence and Diffusion, American Meteorological Society, Boston, Ma. pp. 281-284.

Hsu, C., E.Y. Hsu and R.L. Street (1981) On the structure of turbulent flow over a progressive water wave: theory and experiment in a transformed, wave-following 
co-ordinate system. Journal of Fluid Mechanics vol. 105, pp. 87-117.

Hsu, C. and E.Y. Hsu (1983) On the structure of turbulent flow over a progressive water wave: theory and experiment in a transformed wave-following coordinate system. Part 2. Journal of Fluid Mechanics, vol. 131, pp. 123-153.

Huang, N.E. (1979) On surface drift currents in the ocean. J. Fluid Mech. Vol. 91, part 1, pp. 191-208.

Hunt, J. C. R. (1984) Turbulence Structure and Turbulent Diffusion Near Gas-liquid Interfaces. in Gas Transfer at Water Surfaces, W. Brutsaert and G. H. Jirka eds. D. Reidel Publishing Co. pp. 67-82.

Johnson, H.K. and H.J. Vested (1992) Effects of water waves on wind shear stress for current modeling. Journal of Atmospheric and Oceanic Technology, vol. 9, pp. 850-861.

Kaimal, J.C., J.C. Wyngaard, and D.A. Haugen (1968) Deriving Power Spectra from a Three-Component Sonic Anemometer. Journal of Applied Meteorology, vol. 7, pp. 827837.

Katsaros, K.B., W.J. Shaw and K. Drummond (1978) Laboratory experiments on the interaction between surface waves and free convection in water. in:Turbulent fluxes through the sea surface, wave dynamics, and prediction. A. Favre and K. Hasselman, editors, Plenum Press.

Kenyon, K.E. (1969) Stokes drift for random gravity waves. Journal of Geophysical Research 74, pp. 6991-6994.

Kitaigorodskii, S.A. and M.A. Donelan (1984) Wind-wave Effects on Gas Transfer. in Gas transfer at Water Surfaces, W. Brutsaert and G. H. Jirka eds. D. Reidel Publishing Co. pp. 147-170.

Kiya, M., Y.Suzuki, M. Arie and M. Hagino (1982) A contribution to the free-stream turbulence effect on the flow past a circular cylinder. J. Fluid Mech. vol. 115, pp. 151-164.

Kunze, E, A.J. Williams III and M.G. Briscoe (1990) Observations of Shear and Vertical Stability From a Neutrally Buoyant Float. Journal of Geophysical Res. vol. 95, No.C10, two part paper, pp. 18,111-18,125 18,127-18,142.

Langmuir, I. (1938) Surface Motion of Water Induced by Wind. Science, vol. 87 No. 2250, pp. 119-123.

Ledwell, J.R., A.J. Watson, and C.S. Law (1993) Evidence for Slow Mixing Across the Pycnocline from an Open-Ocean Tracer-Release Experiment. Nature, Vol. 364, pp. 701- 
703.

Leader, D.E. (1994) Kalman Filter Estimation of Underwater Vehicle Position and Attitude Using a Doppler Velocity Aided Inertial Motion Unit. M.I.T.-W.H.O.I. Ocean Engineer's thesis, $106 \mathrm{p}$.

Leibovich, S. (1983) The form and dynamics of Langmuir circulations. Ann. Rev. Fluid Mech. 15, pp. 391-427.

Li, M., and D. Farmer (1993) Oil Submersion in a Wind Driven Sea. Oceans '93, pp. I-330-333.

Lienhard, J.H. and C.W. Van Atta (1990) The decay of turbulence in thermally stratified flow. Journal of Fluid Mechanics, vol. 210, pp. 57-112.

Ling, S.C. (1993) Effect of Breaking Waves on the Transport of Heat and Vapor Fluxes from the Ocean. Journal of Physical Oceanography vol. 23, Nov. pp. 2360-2372.

Loder, J.W. and J.M. Hamilton (1991) Degradation of some mechanical current meter measurements by high-frequency mooring or wave motion. IEEE Journal of Oceanic Engineering, v16, No. 4, pp. 343-349.

Longuet-Higgins, M.S., D.E. Cartwright, and N.D. Smith (1963) Observations of the Directional Spectrum of Sea Waves Using the Motions of a Floating Buoy. Ocean Wave Spectra, Prentice-Hall, pp. 111-136.

Longuet-Higgins, M.S. (1983) Bubbles, breaking waves and hyperbolic jets at a free surface. Journal of Fluid Mechanics, vol. 127, pp. 103-121.

Longuet-Higgins, M.S. (1987) Lagrangian moments and mass transport in Stokes waves. Journal of Fluid Mechanics, Vol. 179, pp. 547-555.

Lygre, A., and H.E. Krogstad (1986) Maximum Entropy Estimation of the Directional Distribution in Ocean Wave Spectra. Journal of Physical Oceanography, Vol. 16, pp. 2052-2060.

Madsen, O.S. (1977) A realistic model of the wind-induced Ekman boundary layer. Journal of Physical Oceanography. 7. pp 248-255.

McKern, R.A. (1968) A study of transformation algorithms for use in a digital computer. M.S. Thesis, MIT.

Meirovitch, L. (1975) Elements of Vibration Analysis. McGraw-Hill Co. 495 p.

Mellor, G.L. and T. Yamada (1982) Development of a turbulence closure model for 
geophysical fluid problems. Reviews of Geophysics and Space Physics, vol. 20, No. 4, pp. 851-875.

Melville, W.K. (1992) The role of wave breaking in air-sea interaction. lecture presented at $18 \mathrm{th}$ International Congress of Theoretical and Applied Mechanics, Haifa, Israel, August, 1992.

Menemenlis, D. and D.M. Farmer (1992) Acoustical measurement of current and vorticity beneath ice. Journal of Atmospheric and Oceanic Technology. vol. 9, pp. 827-849.

Menemenlis, D. (1994) Line-averaged measurement of velocity fine structure in the ocean using acoustical reciprocal transmission. Int. J. Remote Sensing, Vol. 15, No. 2, pp. 267281.

Menemenlis, D. and D.M. Farmer (1995) Path-averaged measurements of turbulence beneath ice in the Arctic. J. Geophysical Res. Vol. 100, No. C7, pp. 13655-13663.

Mestayer, P.G., F.H. Champagne, C.A. Friehe, J.C. La Rue and C.H. Gibson (1978) Estimation of the fluxes over the ocean by the covariance and dissipation methods. in: Turbulent fluxes through the sea surface, wave dynamics, and prediction. A. Favre and K. Hasselman, editors, Plenum Press.

Mitsuyasu, H. (1985) A Note on the Momentum Transfer From Wind to Waves. Journal of Geophysical Research, Vol. 90, No. C2, pp. 3343-3345.

Monin, A. S. and A. M. Yaglom (1971) Statistical Fluid Mechanics, MTT Press. 769p.

Montgomery, E.T. and M.J. Santala (1989) The surface acoustic shear sensor (SASS) as used during the shelf mixed layer experiment. WHOI report WHOI-89-34 20p.

Morrison, A.T., A.J. Williams, and M. Martini (1993) Calibration of the BASS Acoustic Current Meter With Carrageenan Agar. Oceans '93, pp. III-143-148.

Muller, P., R. Lien, and R. Williams (1988) Estimates of Potential Vorticity at Small Scales in the Ocean. J. Phys. Oceanography. Vol. 18, pp. 401-416.

Munz, C. and P. V. Roberts (1984) The Ratio of Gas-phase to Liquid-phase Mass Transfer Coefficients in Gas-liquid Contacting Processes. in Gas Transfer at Water Surfaces, W. Brutsaert and G. H. Jirka eds. pp. 35-45.

Nagel, P. (1992) Non-causal complementary filters. Signal Processing, v. 29, pp. 151-164.

Nepf, H.M. (1992) The Production and Mixing Effects of Langmuir Circulations. PhD Thesis, Stanford, 134p. 
Neshyba, S., V.T. Neal, and R. Tucker (1975) Rapid high-resolution in situ sampling of the internal wave on a density step. Journal of Geophysical Res. vol. 80, No. 3, pp. 312-313.

Newman, J. N. (1982) Marine Hydrodynamics, MIT Press. 402 p.

Niiler, P.P. (1975) Deepening of the wind-mixed layer. Journal of Marine Research, 33, pp. $405-422$.

Oertel, H. (1990) Wakes behind blunt bodies. Ann. Rev. Fluid Mech. 22, pp. 539-64.

Oost, W.A., C.W. Fairall, J.B. Edson, S.D. Smith, R.J. Anderson, J.A.B. Bills, K.B. Datsaros and J. SeCosmo (1994) Flow Distortion Calculations and Their Application in HEXMAX. Journal of Atmospheric and Oceanic Technology, vol. 11, pp 366-386.

Pio, R.L. (1966) Euler Angle Transformations. IEEE Transactions on Automatic Control, vol. AC-11, No. 4, October, pp. 707-715.

Pollard, R.T. (1973) Interpretation of near-surface current meter observations. Deep-Sea Research, 20, pp. 261-268.

Pollard, R.T. (1977) Observations and theories of Langmuir circulations and their role in near surface mixing. In: $A$ Voyage of Discovery: $G$. Deacon 70th Anniversary Volume, $\mathrm{M}$. Angel, editor, Pergamon Press, Now York, pp. 235-251.

Potter, M.C. and J.F. Foss (1982) Fluid Mechanics, 588p.

Poulain, P, (1990) Near-inertial and diurnal motions in the trajectories of mixed layer drifters. Journal of Marine Research, 48, pp. 793-823.

Price, J.F., C.N.K. Mooers and J.C Van Leer (1978) Observation and simulation of storm-Induced Mixed-Layer Deepening. Journal of Physical Oceanography vol. 8, pp. 582-599.

Price, J.F., R.A. Weller and R.R. Schudlich (1987) Wind-driven Ocean Currents and Ekman Transport. Science. 238, pp. 1534-1538.

Richman, J.G., R.A. Szoeke and R.E.Davis (1987) Measurements of Near-Surface Shear in the Ocean, Journal of Geophysical Research, 92, pp. 2851-2858.

Roberts, E., D. Last and G. Roberts (1991) Accurate current measurement using drogued buoys employing decca navigator and argos. IEEE Journal of Oceanic Engineering, v16, No.4, pp. 307-312.

Rohsenow, W.M. and H. Choi (1961) Heat, Mass, and Momentum Transfer, Prentice- 
Hall. 537p.

Rossby, T. (1975) An Oceanic Vorticity meter. Journal of Marine Research, 33, pp. 213222.

Salmon, R., (1990) The thermocline as an "internal boundary layer". Journal of Marine Research, 48, pp. 437-469.

Sanderson, B.G. and A. Okubo (1988) Diffusion by internal waves. Journal of Geophysical Research, vol. 93, pp. 3570-3582.

Santala, J.J. and A.J. Williams III (1990) Estimates of directional spectra from the surface acoustic shear sensor (SASS). Oceans'90 Conference Proceedings, pp. 41-45.

Santala, M. J. (1991) Surface-Referenced Current Meter Measurements, P.H.D. Thesis, M.I.T.-W.H.O.I..

Santala, M.J. and E.A. Terray (1992) A technique for making unbiased estimates of current shear from a wave-follower, Deep-Sea Research, vol. 39, No. 3/4, pp. 607-622.

Saunders, P.M. (1980) Overspeeding of a savonious rotor. Deep-Sea Research, 27A, pp. 755-759.

Schlichting, H. (1979) Boundary-Layer Theory, McGraw-Hill. 817p.

Schmidt, G.T. (1978) Strapdown Inertial Systems---Theory and Applications Introduction and Overview. in NATO Advisory Group for Aerospace Research and Development (AGARD) Lecture Series No. 95: Strapdown Inertial Systems, Technical Editing and Reproduction, Ltd., London, pp. 1-1-10.

Simpson, J.P. (1979) The effects of fouling on electromagnetic current meters. Exposure vol. 7 no. 2 pp. $1-4$.

Smith, S.D. (1988) Coefficients for sea surface wind stress, heat flux, and wind profiles as a function of wind speed and temperature. Journal of Geophysical research, vol. 93, pp. $15,467-15,472$.

Stigebrandt, A. (1976) Vertical diffusion driven by internal waves in a sill fjord. Journal of Physical Oceanography. Vol. 6, pp. 486-495.

Stillinger, D.C., K.N. Helland and C.W. Van atta (1983) Experiments on the transition of homogeneous turbulence to internal waves in a stratified fluid. Journal of Fluid Mechanics, vol. 131, pp. 91-122.

Stull, R.B. (1988) Boundary Layer Meteorology. Kluwer Academic Publishers, 666p. 
Tennekes, H., and J.L. Lumley (1972) A First Course in Turbulence. MIT Press, 300p.

Terray, E. A., A, J. Williams III, and B. H. Brumley, Observation of Shear-free Turbulence Beneath Whitecaps in the Marine Surface Layer, internal WHOI document.

Thomas, G.B. (1968) Calculus and Analytic Geometry. Addison-Wesley Publishing 818p.

Thorpe, S.A. (1975) The excitation, dissipation, and interaction of internal waves in the deep ocean. Journal of Geophysical Res. vol. 80, No. 3, pp. 328-338.

Thorpe, S.A. and P.N. Humphries (1980) Bubbles and breaking waves. Nature vol. 283, pp. 463-465.

Thorpe, S.A. (1982) On the clouds of bubbles formed by breaking wind-waves in deep water, and their role in air-sea gas transfer. Phil. Trans. R. Soc. Lond. A 304, pp. $155-210$.

Tochko, J. S. (1978) A Study of the Velocity Structure in a Marine Boundary Layer-Instrumentation and Observations, P.H.D. Thesis, M.I.T.-W.H.O.I..

Triantafyllou, G.S., M.S. Triantafyllou and C. Chryssostomidis (1986) On the formation of vortex streets behind stationary cylinders. Journal of Fluid Mechanics. vol. 170, pp. $461-477$.

Trivett, D.A., E.A. Terray and A.J. Williams, IHI (1991) Error analysis of an acoustic current meter. IEEE Journal of Oceanic Engineering, vol. 16, No. 4, pp. 329-337.

Trivett, D. A. (1991) Diffuse Flow from Hydrothermal Vents. M.I.T.-W.H.O.I. P.H.D. Thesis.

Trowbridge, J.H. (1992) A simple description of the deepening and structure of a stably stratified flow driven by a surface stress. Journal of Geophysical Res. Oceans vol. 97, No. C10, pp. 15529-15543.

Tsinober, A. E. Kit and M. Teitel (1987) On the relevance of the potential-difference method for turbulence measurements. Journal of Fluid Mechanics, 175, pp. 447-461.

Turner, J.S. (1973) Buoyancy effects in fluids. Cambridge University Press, 367p.

VanBronkhorst, A. (1978) Strapdown System Algorithms. in NATO Advisory Group for Aerospace Research and Development (AGARD) Lecture Series No. 95: Strapdown Inertial Systems, Technical Editing and Reproduction, Ltd., London, 1978.

Wannop,K.A. and A.J. Williams III (1987) The development of BASS Benthic Acoustic Stress Sensor. WHOI technical memorandum WHOI-1-87 87p. 
Watson, N.R. (1966) Some measurements of turbulence in altocumulus clouds. Quart. J. Roy. Meteor. Soc., 93, pp. 227-236.

Weller, R. A., and R. E. Davis (1980) A Vector Measuring Current Meter, Deep Sea Res., 27, pp. 565-581.

Weller, R.A. (1985) Three-dimensional flow in the upper ocean. Science, 227, pp. 1552-1556.

Weller, R.A. and J.F. Price (1988) Langmuir circulation within the oceanic mixed layer. Deep-sea Research, vol.35, No.5, pp. 711-747.

Williams, A. J.III, J. S. Tochko, R. L. Koehler, W. D. Grant, T. F. Gross, and C. V. R. Dunn (1987) Measurement of Turbulence in the Oceanic Bottom Boundary Layer with an Acoustic Current Meter Array, Journal of Atmospheric and Oceanic Technology, vol. 4, No. 2, pp. 312-327, June.

Williams, A.J.III (1993) A Free-Dritting Measurement of Mid-Water Mixing by an Acoustic Current Meter Array in NATRE. Oceans '93, pp. III-131-136.

Williams, A.J.III, E.A. Terray, F.T. Thwaites, and J. H. Trowbridge (1994) Acoustic Vorticity Meter for Benthic Boundary Layer Flow Measurements. Oceans '94, pp. III250-253.

Williamson, D.H.K. (1985) Sinusoidal flow relative to circular cylinders. Journal of Fluid Mechanics. 155, pp. 141-174.

Wu, Jin (1975) Wind-induced drift currents. Journal of Fluid Mechanics, 68, pp. 49-70.

Wu, Jin (1983) Sea-surface drift currents induced by wind and waves. Journal of Physical Oceanography, 13, pp. 1441-1451.

Wunsch, C. (1975) Deep ocean internal waves: What do we really know? Journal of Geophysical Res. vol. 80, No. 3, pp. 339-343.

Yelland, M.J., P.K. Taylor, I.E. Consterdine, and M.H. Smith (1994) The use of the inertial dissipation Technique for shipboard wind stress determination. Journal of Atmospheric and Oceanic Technology, vol. 11, pp. 1093-1108.

Yoon, K. and Z. Warhaft (1990) The evolution of grid-generated turbulence under conditions of stable thermal stratification. Journal of Fluid Mechanics 215, pp. 601-638. 